This item was submitted to Loughborough's Research Repository by the author.

Items in Figshare are protected by copyright, with all rights reserved, unless otherwise indicated.

\title{
Pathwise properties of random quadratic mapping
}

PLEASE CITE THE PUBLISHED VERSION

PUBLISHER

(C) Peng Lian

LICENCE

CC BY-NC-ND 4.0

REPOSITORY RECORD

Lian, Peng. 2019. "Pathwise Properties of Random Quadratic Mapping”. figshare.

https://hdl.handle.net/2134/6628. 
This item was submitted to Loughborough's Institutional Repository (https://dspace.lboro.ac.uk/) by the author and is made available under the following Creative Commons Licence conditions.

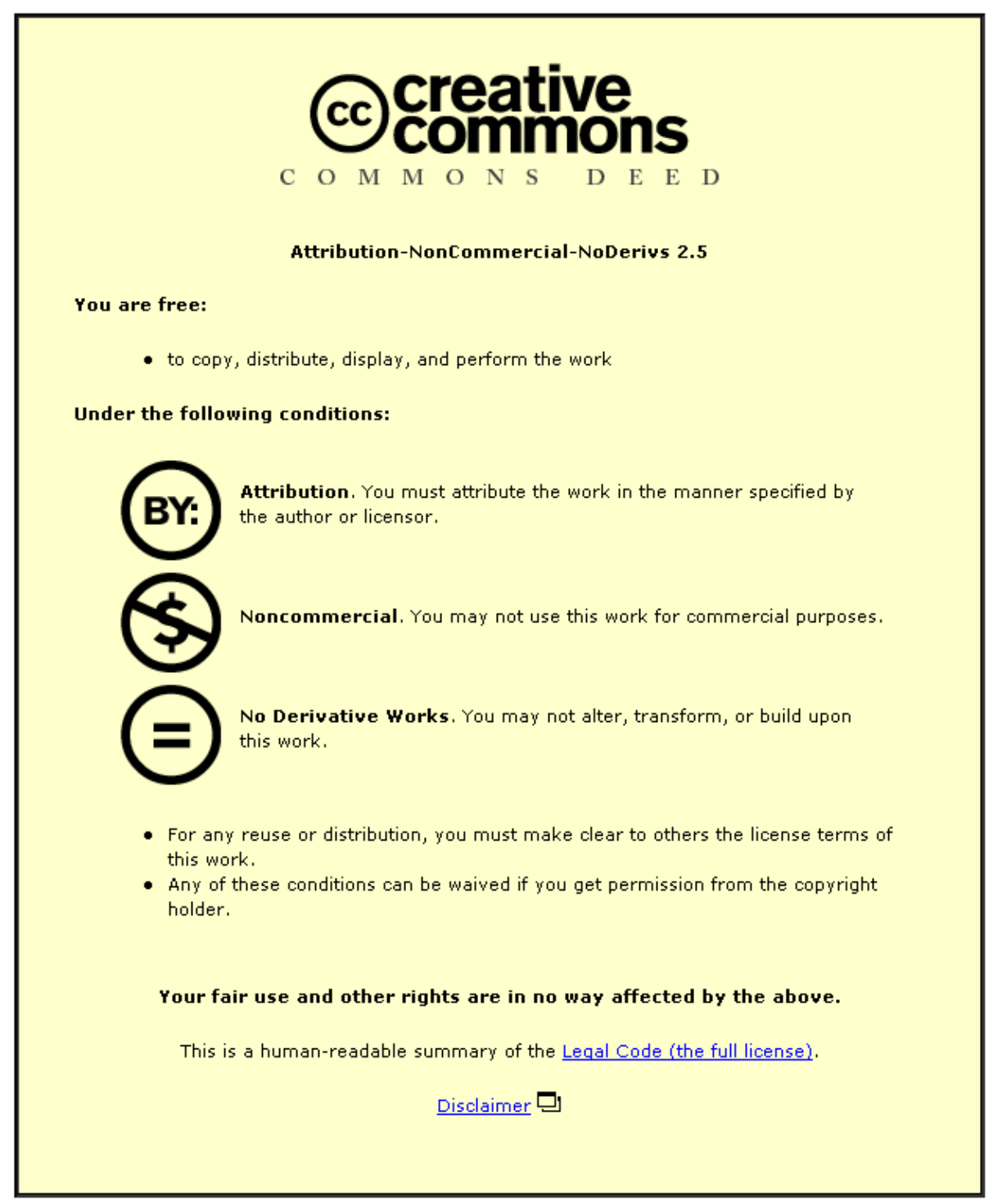

For the full text of this licence, please go to: http://creativecommons.org/licenses/by-nc-nd/2.5/ 
Pathwise properties of random quadratic mapping

Peng Lian

(Department of Mathematical Sciences, Loughborough University, LE11 3TU, UK)

September 13, 2010 


\begin{abstract}
In this thesis, we consider the random dynamical system from a sequence of random quadratic mapping $f_{\epsilon_{k}}(x)=\epsilon_{k} x(1-x)$, where $\epsilon_{k}$ can choose $\mu$ or $\lambda$ randomly, where $1<\mu<\lambda \leqslant 1+\sqrt{5}$. That means we consider $X_{k}=f_{\epsilon_{k}} \cdots f_{\epsilon_{1}}\left(X_{0}\right)$, where $\left\{\epsilon_{k}: k \geqslant 1\right\}$ is a sequence with $\epsilon_{k}=\mu$ or $\lambda$ and $X_{0} \in[0,1]$. As to this random dynamical system, we prove the existence of the stationary solution when $1<\mu<\lambda \leqslant 3$ and the existence of random periodic solution of period 2 for $\epsilon_{2 i}=\epsilon_{2 i+1}(i \in \mathbb{Z})$ when $3.00547 \leqslant \mu<\lambda \leqslant 1+\sqrt{5}$.
\end{abstract}

Keywords: stationary solution, random periodic solution, random dynamical systems, random quadratic mapping, pathwise, invariant measure. 


\section{Acknowledgements}

No one can complete a work alone, especially for such a thesis. Then thanks should go to those people who help me complete this research.

Firstly, I'd like to express my deepest and sincerest gratitude to Prof. Huaizhong Zhao, my supervisor, who keeps providing me with his unwavering support during my $\mathrm{PhD}$ study. With profound knowledge and eminent academic ability, He can always solve all kinds of problems which appeared in the process of the research. I very much appreciate his kind help and encouragement in my study and in my life. His perfect personality will benefit me for my future life.

Many thanks also go to Jo Corlett and all other secretaries in department for their help in administration. Meanwhile, all the staff in department are very friendly and they provide me a comfort study environment.

I am greatly thankful to my colleagues Dr. Chunrong Feng, Dr. Bo Zhou, Fajin Wei, Xiaohui Yan, Qingfeng Wang and my friend Min Zhang, Shiyan Guo for helpful discussion in my study and providing support, entertainment to my life.

I would also acknowledge the support from my beloved family. Their love is priceless wealth to me and I will cherish it forever. 


\section{Contents}

1 Introduction 1

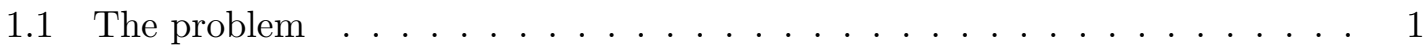

1.2 Random stationary solution . . . . . . . . . . . . . . . . 4

1.3 Random periodic solution of period $2 \ldots \ldots \ldots \ldots$

1.4 Invariant measure . . . . . . . . . . . . . . . . . . . . 5

2 Limit of the pullback random mappings $\quad 6$

2.1 The existence of the limit when $\mu, \lambda \in(1,3] \ldots \ldots \ldots \ldots$

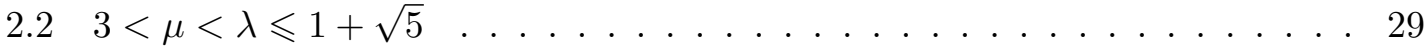

2.2 .1 Limit of pullback. . . . . . . . . . . . . . . . 29

3 Proof of the two main theorems

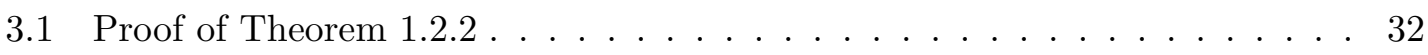

3.2 Proof of Theorem $1.3 .2 \ldots \ldots \ldots \ldots \ldots$

4 Proof of lemmas $\quad \mathbf{3 4}$

4.1 Two general lemmas for $\mu, \lambda \in(1,3] \ldots \ldots \ldots \ldots \ldots$

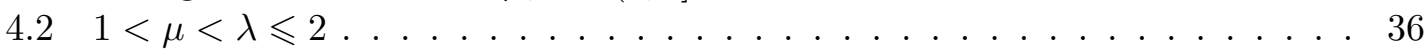

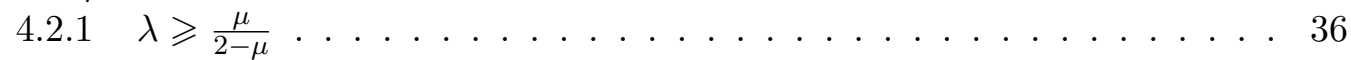

$4.2 .2 \quad \lambda \geqslant \frac{\mu}{(2-\mu)^{2}} \ldots \ldots \ldots \ldots \ldots \ldots \ldots \ldots \ldots \ldots \ldots$

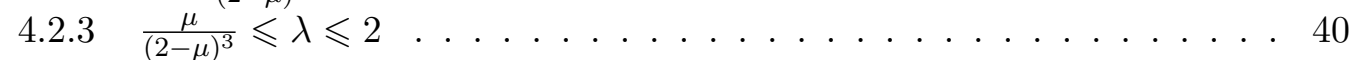

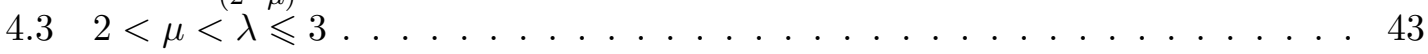

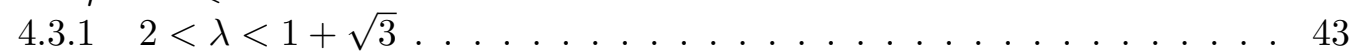

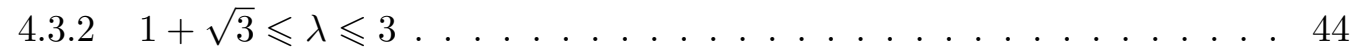

$4.41<\mu \leqslant 2<\lambda \leqslant 3 \ldots \ldots \ldots \ldots \ldots \ldots \ldots$

4.4 .1 Case $\frac{4}{\lambda}<\mu \leqslant 2,2<\lambda \leqslant 3 \ldots \ldots \ldots \ldots$. . . . . . . . . . . . . . . . . 47

4.4 .2 Case $1<\mu<\frac{4}{\lambda}<2<\lambda \leqslant 3 \ldots \ldots \ldots \ldots$. . . . . . . . . . . . . . . . 50

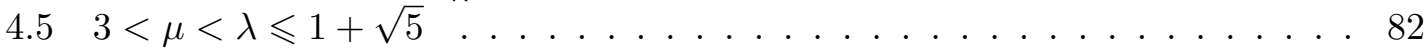




\section{Chapter 1}

\section{Introduction}

\subsection{The problem}

Random dynamical systems are used to model the dynamics when it is influenced or perturbed by some kind of random noises. In 1945, Ulam and Neumann [40] pointed out the importance of random dynamical systems. In the last thirty years, the research of random dynamical systems is further expanded especially in the field of stochastic differential equations and stochastic partial differential equations in a series of work such as $[4,23,24,28,31]$. Pathwise stationary solution and random periodic solution are two central concepts in the study of random dynamical systems $[4,13,14,21,20,29,31,36$, $37,42,43,44]$. To study them is key towards understanding the longtime behavior of the random dynamical systems and their local and global topological structure. In this thesis, we will consider a random dynamical system which is comprised by a family of quadratic functions $\left\{f_{\eta}: 0 \leqslant \eta \leqslant 4\right\}$, where $f_{\eta}$ is the map on the interval $[0,1]$, defined by $f_{\eta}(x)=\eta x(1-x)$. Given a pair of parameter values $\mu<\lambda$ and a number $\gamma \in(0,1)$, we consider a sequence of maps $\left\{f_{\epsilon_{k}}: k \geqslant 0\right\}$ with $P\left(f_{\epsilon_{k}}=f_{\mu}\right)=\gamma, P\left(f_{\epsilon_{k}}=f_{\lambda}\right)=1-\gamma$. Here $\epsilon_{k}$ is a random variable with Bernoulli distribution:

$$
P\left(\epsilon_{k}=\mu\right)=1-P\left(\epsilon_{k}=\lambda\right)=\gamma .
$$

Define $\Omega=\left\{\left(\cdots, \epsilon_{-2}, \epsilon_{-1} ; \epsilon_{0}, \epsilon_{1}, \epsilon_{2}, \cdots \epsilon_{k}, \cdots\right): \epsilon_{k}=\mu\right.$ or $\left.\lambda, k=\cdots,-2,-1,0,1,2, \cdots\right\}$ as the sample space of the underlying probability space for the random dynamical system. Denote $\omega=\left(\cdots, \epsilon_{-2}, \epsilon_{-1} ; \epsilon_{0}, \epsilon_{1}, \epsilon_{2}, \cdots \epsilon_{k}, \cdots\right)$ and define the metric dynamical system $\theta:\{\cdots,-2,-1,0,1,2, \cdots\} \times \Omega \rightarrow \Omega$ by $\theta^{k} \omega=\left(\cdots, \epsilon_{k-2}, \epsilon_{k-1} ; \epsilon_{k}, \epsilon_{k+1}, \cdots\right)$. We define the evaluation map at position 1: $e: \Omega \rightarrow\{\mu, \lambda\}$ by the formula $e(\omega)=\epsilon_{0}$. It is obvious that $e\left(\theta_{k} \omega\right)=\epsilon_{k} \in\{\mu, \lambda\}$. Moreover, for a given random variable $X_{0} \in[0,1]$, we consider

$$
\begin{gathered}
\Phi_{0}(\omega, \cdot)=i d, \quad \Phi_{1}(\omega, \cdot)=f_{e(\omega)}(\cdot) . \\
\Phi_{k}(\omega, \cdot)=\Phi_{k-1}(\theta \omega) \circ \Phi_{1}(\omega, \cdot), \quad X_{k}=\Phi_{k}\left(\omega, X_{0}\right) .
\end{gathered}
$$

with $X_{0}$ independent of $\left\{\epsilon_{k}: k \geqslant 1\right\}$. We can check that $\Phi$ defined above satisfies the condition of random dynamical system given in Def 1.1.1 below. Firstly, we recall the definition of random dynamical systems [4]. 
Definition 1.1.1 Let $(\Omega, \mathcal{F}, \mathbb{P})$ be a probability space and $\theta: \Omega \rightarrow \Omega$ be a measurable transformation which preserves the measure $\mathbb{P}$, i.e. for each measurable set $A \in \mathcal{F}$,

$$
\mathbb{P}\left(\theta^{-1} A\right)=\mathbb{P}(A) .
$$

A discrete time measurable random dynamical system over $(\Omega, \mathcal{F}, \mathbb{P}, \theta)$ on a topological space $X$ with Borel $\sigma$-algebra $\mathcal{B}$ is a measurable mapping

$$
\Phi: \quad(n, \omega, x) \in \mathbb{Z} \times \Omega \times X \rightarrow \Phi(n, \omega)(x) \in X
$$

satisfying the cocycle property: $\Phi(0, \omega)=i d$ and

$$
\Phi(n+m, \omega)=\Phi\left(m, \theta^{n} \omega\right) \circ \Phi(n, \omega)
$$

for every $n, m \in \mathbb{Z}$ and almost every $\omega \in \Omega$.

Here it is easy to check the probability $\mathbb{P}$ in our problem satisfies Definition 1.1.1, i.e. for each $\omega$, the number of $\mu$ and the number of $\lambda$ in $\theta^{-1} \omega$ should be equal to those in the set $A$, so we can get the equation (1.2). On the other hand, it is obvious to see the cocycle property is satisfied by definition of $\Phi$ in (1.1).

These random dynamical systems, defined from a sequence of random mappings, comprise a mathematically rich class of Markov processes. There has been many research on iterated random quadratic mapping on $[0,1]$ mainly based on seminal work of Dubins and Freedman [19]. Bhattacharya and Rao [12] proved that there exists a unique invariant probability $\pi$ for the process $X_{k}$ on $(0,1)$ when $1<\mu<\lambda \leqslant 3$ and the support $S(\pi)$ of $\pi$ is a Cantor subset of $\left[p_{\mu}, p_{\lambda}\right]\left(p_{\mu}\right.$ and $p_{\lambda}$ are fixed points of $f_{\mu}$ and $f_{\lambda}$ respectively similar as (1.5)) when $f_{\lambda}\left(p_{\mu}\right)>f_{\mu}\left(p_{\lambda}\right)(1<\mu<\lambda \leqslant 2)$. Bhattacharya and Majumdar [10] extended the above result to the case $3<\mu<\lambda<1+\sqrt{5}$. Unfortunately, there is a gap in their proof. They use that $\left[\beta_{\mu}, \beta_{\lambda}\right]$ is an invariant interval, where $\beta_{\mu}$ and $\beta_{\lambda}$ are defined similar as (1.6) when $\eta=\mu$ or $\lambda$. Actually, this is not true in general. One needs more conditions for the result to hold. While our main interest here is to study the pathwise stationary solution and the random periodic solutions of the random mapping. We can see naturally both stationary solution and random periodic solution give an invariant measure.

To see the motivation of random stationary solution and random periodic solution, firstly recall some well-known elementary result about the fixed point and its stability of the deterministic quadratic map [8, 30, 32, 22, 38, 39],

$$
f_{\eta}(x)=\eta x(1-x), \quad x \in[0,1] .
$$

At the beginning, this family of quadratic maps arose as a sample models of population biology. This model actually posses very interesting dynamics such as periodic solutions, bifurcations and so on $[1,15,6,41,25]$. When $\eta \in[0,4]$, this function is sometimes called an iterated map function, since it maps $x \in[0,1]$ to another value of $x$ in the same range $[0,1]$. An $x_{\eta}^{\star}$ satisfying

$$
x_{\eta}^{\star}=f_{\eta}\left(x_{\eta}^{\star}\right) .
$$

is called a fixed point of the iterated map. The subscript $\eta$ indicates that $x^{\star}$ depends on the value of $\eta$. For the logistic map (1.4), there are two fixed points generally:

$$
x_{\eta}^{\star}=0, \quad x_{\eta}^{\star}=1-\frac{1}{\eta}=: p_{\eta} .
$$


When $\eta \leqslant 1, x_{\eta}^{\star}=0$ is the only stable fixed point. When $\eta>1,0$ and $p_{\eta}$ are both in the range of interest between 0 and 1 . For $1 \leqslant \eta \leqslant 3$, there is only one stable fixed point $p_{\eta}=1-\frac{1}{\eta}$. Moreover

$$
\begin{cases}p_{\eta} \in\left(0, \frac{1}{2}\right] & \text { when } \eta \in(1,2] \\ p_{\eta} \in\left(\frac{1}{2}, 1\right) & \text { when } \eta \in(2,3]\end{cases}
$$

and for any $x \in(0,1), f^{k}(x) \rightarrow x^{\star}$, as $k \rightarrow \infty$.

Considering inherent uncertainty in many kinds of systems, a randomly perturbed system is a useful model addressing uncertainty as well. Random dynamical systems generated by monotone random maps can be applied in economics field, for example, as model of survival or models of optimal transition of stocks from one period to the next under uncertainty [10]. However, when $1<\mu<\lambda \leqslant 3$, for the random case, it is very different. There is no "fixed point" unless we only apply the same map $f_{\mu}$ or $f_{\lambda}$ on the "fixed point" of $f_{\mu}$ or $f_{\lambda}$ respectively. It is easy to see that starting from any point, when we apply $f_{\lambda}$, the point will move towards the fixed point of $f_{\lambda}$, and when we apply $f_{\mu}$, the point will move towards to the fixed point of $f_{\mu}$. So the situation is very interesting and there is no fixed point in the sense of deterministic dynamical systems. So stationary solution should be understood in the sense of random stationary solution given below.

For $\eta \in(3,1+\sqrt{5}]$, it is known that in this case $f_{\eta}$ has an unstable, or repelling, fixed point $p_{\eta}=1-\frac{1}{\eta}$ which is encompassed by an attractive, or stable, period-two orbit $\left\{\alpha_{\eta}, \beta_{\eta}\right\}$, such that:

$$
\alpha_{\eta}=\frac{\eta+1-\sqrt{(\eta+1)(\eta-3)}}{2 \eta}<p_{\eta}=1-\frac{1}{\eta}<\beta_{\eta}=\frac{\eta+1+\sqrt{(\eta+1)(\eta-3)}}{2 \eta} .
$$

In other words, the iteration alternates between one point and the other. Starting from one of these points, we must iterate twice to return to the same point. The two points constitute an attractor of period two, also called a 2-cycle, given that

$$
\begin{aligned}
& \beta_{\eta}=f\left(\alpha_{\eta}\right)=f\left(f\left(\beta_{\eta}\right)\right), \\
& \alpha_{\eta}=f\left(\beta_{\eta}\right)=f\left(f\left(\alpha_{\eta}\right)\right) .
\end{aligned}
$$

These two points, which are not fixed points of $f$, are fixed points of the function:

$$
F(x)=f(f(x))=f^{2}(x)
$$

Needless to say, the situation in random case is more complicated than the deterministic case and also more complex than the stationary solution for random mapping when $1<$ $\mu<\lambda \leqslant 3$.

Of course, for the deterministic quadratic mapping, further increases in $\eta$ will lead to period $-2^{2}$, period $-2^{3}$ and so on, Occurring at even smaller and smaller increments of $\eta$. When $\eta>3.5699 \ldots$, a region where aperiodic and periodic attractors alternate [22]. Accordingly the random case will also be more and more complicated as the periodicity increases and deserved to be studied in the future. 


\subsection{Random stationary solution}

The main result of this thesis is Theorem 1.2.2 and Theorem 1.3.2 blew. First, we recall the definition of stationary solution.

Definition 1.2.1 A random invariant point (stationary solution) for the random dynamical system $\Phi$ is a random variable $Y^{\star}: \Omega \rightarrow X$ with

$$
\Phi(k, \omega) Y^{\star}(\omega)=Y^{\star}\left(\theta^{k} \omega\right) \mathbb{P}-a . s,
$$

for every $k \in \mathbb{Z}$.

Theorem 1.2.2 Assume $1<\mu<\lambda \leqslant 3$. Then for any $\omega=\left(\cdots, \epsilon_{-k+1}, \cdots, \epsilon_{-1} ; \epsilon_{0}, \epsilon_{1}, \cdots\right)$, there exists $Y^{\star}(\omega) \in(0,1)$, such that $\Phi_{k}(\omega) Y^{\star}(\omega)=Y^{\star}\left(\theta^{k} \omega\right)$ for all $k$. And for any $x \in\left[1-\frac{1}{\mu}, 1-\frac{1}{\lambda}\right]$, we have

$$
\lim _{n \rightarrow \infty} \Phi_{n}\left(\theta^{-n} \omega\right)(x)=Y^{\star}(\omega) .
$$

In order to prove this theorem, we need to use the following Proposition 2.1.6, Proposition 2.1.8 and Proposition 2.1.16. According to the result of these propositions, we have proved that as $n \rightarrow \infty$, the limit of $f_{\epsilon_{-1}} f_{\epsilon_{-2}} \cdots f_{\epsilon_{-n+1}} f_{\epsilon_{-n}}(x)$ exists, denoted by $Y^{\star}(\omega)$. We will prove that $Y^{\star}(\omega)$ gives the stationary solution of the random mapping in Chapter 3 .

\subsection{Random periodic solution of period 2}

The study of periodic solutions of dynamical systems began with Poincare's fundamental work [34]. Poincare-Bendixson Theorem played key role in these studies [9]. Since then there have been a lot of research about the periodic solutions in real world problem, such as Van der Pol equations [35] and Liénard equations [27]. Now, this topic is still one of the most interesting phenomena for deterministic dynamical systems. The current understanding of periodic solutions in a random environment is minimal. Needless to say, to understand random periodic solution is a key to understand many interesting phenomenon in complex systems. In this thesis, we study the random periodic solution for the random quadratic mapping. Inspired by the definition of the random periodic solution of cocycles on a cylinder in Zhao and Zheng's [44] and random periodic solutions for random semi-flow in Feng, Zhao and Zhao [21], we give our definition as follows, for the discrete time case.

\section{Definition 1.3.1 Consider}

$$
\Omega=\left\{\left(\cdots, \epsilon_{-2}, \epsilon_{-1} ; \epsilon_{0}, \epsilon_{1}, \epsilon_{2}, \cdots \epsilon_{k}, \cdots\right): \epsilon_{k}=\mu \text { or } \lambda, k=\cdots,-2,-1,0,1,2, \cdots\right\} .
$$

A random dynamical system

$$
\Phi: \quad(n, \omega, x) \in \mathbb{Z} \times \Omega \times X \rightarrow \Phi(n, \omega)(x) \in X
$$

given in Definition 1.1.1. A random periodic solution of period 2 is a pair of the random variable $\{\alpha(\omega), \beta(\omega)\}$ such that when $n$ is even

$$
\Phi_{n}(\omega, \alpha(\omega))=\alpha\left(\theta^{n}(\omega)\right)
$$




$$
\Phi_{n}(\omega, \beta(\omega))=\beta\left(\theta^{n}(\omega)\right)
$$

and when $n$ is odd,

$$
\begin{aligned}
& \Phi_{n}(\omega, \alpha(\omega))=\beta\left(\theta^{n}(\omega)\right) \\
& \Phi_{n}(\omega, \beta(\omega))=\alpha\left(\theta^{n}(\omega)\right) .
\end{aligned}
$$

When we compose the condition $\epsilon_{2 i}=\epsilon_{2 i+1}, i \in \mathbb{Z}$ or $\epsilon_{2 i}=\epsilon_{2 i-1}, i \in \mathbb{Z}$ on $\omega$. Now we consider $\Omega^{\star}=\left\{\omega=\left(\cdots, \epsilon_{-2}, \epsilon_{-1} ; \epsilon_{0}, \epsilon_{1}, \cdots\right)\right.$ with $\epsilon_{2 i}=\epsilon_{2 i+1}$ for all $i=0, \pm 1, \pm 2$. $\cdots$ or $\epsilon_{2 i}=$ $\epsilon_{2 i-1}$ for all $\left.i=0, \pm 1, \pm 2 . \cdots\right\}$. Define $\theta$ as before. Then $\theta: \Omega^{\star} \rightarrow \Omega^{\star}$.

Theorem 1.3.2 Assume $3.00547 \leqslant \mu<\lambda \leqslant 1+\sqrt{5}$. For $\omega \in \Omega^{\star}$, there exist random periodic solution of period $2, \alpha(\omega)$ and $\beta(\omega)$, which satisfy (1.9), (1.10), (1.11) and (1.12).

The proof of this theorem will also be given in Chapter 3 .

\subsection{Invariant measure}

It is well known that stationary solution supports corresponding invariant measure. Since we have proved the existence of pathwise stationary solution and random periodic solution, we can prove there is an invariant measure for this random dynamical system $[7,10,11,12]$. According to the definition of invariant measure [4], we will give the following remark.

Remark 1.4.1 It is well known that the random dynamical systems $\Phi_{n}$ has an invariant measure $\nu$ iff the random measure $\nu_{\omega}$ in factorization of the measure $\nu$ satisfies

$$
\Phi(n, \omega) \nu_{\omega}=\nu_{\theta^{n} \omega} \quad \mathbb{P}-\text { a.s. }
$$

When $1<\mu<\lambda \leqslant 3$, we denote the stationary solution by $Y^{\star}(\omega)$. Let $\nu_{\omega}=\delta_{Y^{\star}(\omega)}$, Dirac measure. Then it is easy to see (1.13) holds as

$$
\Phi(n, \omega) \nu_{\omega}=\Phi(n, \omega) \delta_{Y^{\star}(\omega)}=\delta_{\Phi(n, \omega) Y^{\star}(\omega)}=\delta_{Y^{\star}\left(\theta^{n} \omega\right)}=\nu_{\theta^{n} \omega} .
$$

When $3.00547 \leqslant \mu<\lambda \leqslant 1+\sqrt{5}$, for the periodic solution $\{\alpha(\omega), \beta(\omega)\}$ of period 2 , let $\nu_{\omega}=\frac{1}{2} \delta_{\alpha(\omega)}+\frac{1}{2} \delta_{\beta(\omega)}$. Then we can see that

(i) When $n=1$,

$$
\begin{aligned}
\Phi(1, \omega) \nu_{\omega} & =\frac{1}{2} \Phi(1, \omega) \delta_{\alpha(\omega)}+\frac{1}{2} \Phi(1, \omega) \delta_{\beta(\omega)} \\
& =\frac{1}{2} \delta_{\Phi(1, \omega) \alpha(\omega)}+\frac{1}{2} \delta_{\Phi(1, \omega) \beta(\omega)} \\
& =\frac{1}{2} \delta_{\beta\left(\theta^{1} \omega\right)}+\frac{1}{2} \delta_{\alpha\left(\theta^{1} \omega\right)}=\nu_{\theta^{1} \omega} .
\end{aligned}
$$

(ii) When $n=2$,

$$
\begin{aligned}
\Phi(2, \omega) \nu_{\omega} & =\frac{1}{2} \Phi(2, \omega) \delta_{\alpha(\omega)}+\frac{1}{2} \Phi(2, \omega) \delta_{\beta(\omega)} \\
& =\frac{1}{2} \delta_{\alpha\left(\theta^{2} \omega\right)}+\frac{1}{2} \delta_{\beta\left(\theta^{2} \omega\right)}=\nu_{\theta^{2} \omega} .
\end{aligned}
$$

That is to say (1.13) also holds for $\omega \in \Omega^{\star}$. 


\section{Chapter 2}

\section{Limit of the pullback random mappings}

In order to get the existence of the stationary solution for the random mapping, we will prove that pullback $\Phi\left(n, \theta^{-n} \omega\right) x$ converges to a limit as $n \rightarrow \infty$, and the limit does not depend on $x$ for any $1<\mu<\lambda \leqslant 3$.

\subsection{The existence of the limit when $\mu, \lambda \in(1,3]$}

For any $\omega \in \Omega$ and $x \in\left[1-\frac{1}{\mu}, 1-\frac{1}{\lambda}\right]$, we will check whether or not the limit of $f_{\epsilon_{-1}} f_{\epsilon_{-2}} \cdots f_{\epsilon_{-k}}(x)$ exists as $k \rightarrow \infty$.

The proof is surprisingly complicated and difficult, We break it into 3 cases: $1<\mu<$ $\lambda \leqslant 2,2<\mu<\lambda \leqslant 3,1<\mu \leqslant 2<\lambda \leqslant 3$. Note when $1<\mu<\lambda \leqslant 3, p_{\mu}=1-\frac{1}{\mu}$ and $p_{\lambda}=1-\frac{1}{\lambda}$ are two attracting points of $f_{\mu}$ and $f_{\lambda}$ respectively. Moreover, we can notice

$$
\begin{cases}f_{\mu}^{\prime}(x)<1 & \text { when } x \in\left(\frac{1}{2}-\frac{1}{2 \mu}, \frac{1}{2}+\frac{1}{2 \mu}\right), \\ f_{\lambda}^{\prime}(x)<1 & \text { when } x \in\left(\frac{1}{2}-\frac{1}{2 \lambda}, \frac{1}{2}+\frac{1}{2 \lambda}\right) .\end{cases}
$$

Since $\left[\frac{1}{2}-\frac{1}{2 \lambda}, \frac{1}{2}+\frac{1}{2 \lambda}\right] \subset\left[\frac{1}{2}-\frac{1}{2 \mu}, \frac{1}{2}+\frac{1}{2 \mu}\right]$, so for any $x \in\left[\frac{1}{2}-\frac{1}{2 \lambda}, \frac{1}{2}+\frac{1}{2 \lambda}\right], f_{\mu}^{\prime}(x)<1$ and $f_{\lambda}^{\prime}(x) \leqslant 1$. The key in the deterministic case is that the modular derivative of each map is less than 1 in the neighborhood of the fixed point. The dynamical system will definitely move into the neighborhood of the fixed point. However, in the random case, $f_{\lambda}$ makes the process moving towards its fixed point $p_{\lambda}$ and $f_{\mu}$ makes the process moving towards $p_{\mu}$. As a result, the process may move out of the interval $\left[\frac{1}{2}-\frac{1}{2 \lambda}, \frac{1}{2}+\frac{1}{2 \lambda}\right]$. So the derivative of one step map may not have modular smaller than 1 . The idea is to consider several steps together to make the modular derivative of the composited map smaller than 1 . We start with the following easy case.

Proposition 2.1.1 Assume $1<\mu<\lambda \leqslant 3$. If there are only finitely many $\lambda^{\prime} s$ in $\left(\epsilon_{-1} \epsilon_{-2} \cdots\right)$, then for any $x$,

$$
\lim _{k \rightarrow \infty} f_{\epsilon_{-1}} f_{\epsilon_{-2}} \cdots f_{\epsilon_{-k}}(x)=f_{\epsilon_{-1}} f_{\epsilon_{-2}} \cdots f_{\epsilon_{-m}}\left(p_{\mu}\right) .
$$


Here $m$ is an integer such that $\epsilon_{-j}=\mu$ for all $j>m$. If there are only finitely many $\mu^{\prime} s$ in $\left(\epsilon_{-1} \epsilon_{-2} \cdots\right)$, then

$$
\lim _{k \rightarrow \infty} f_{\epsilon_{-1}} f_{\epsilon_{-2}} \cdots f_{\epsilon_{-k}}(x)=f_{\epsilon_{-1}} f_{\epsilon_{-2}} \cdots f_{\epsilon_{-\tilde{m}}}\left(p_{\lambda}\right) .
$$

Here $\tilde{m}$ is an integer such that $\epsilon_{-j}=\lambda$ for all $j>\tilde{m}$.

The proof of this lemma is obvious, following the result for the deterministic case we mentioned in the above section 1.1.

If there are infinitely many $\mu$ and infinitely many $\lambda$ in $\left(\epsilon_{-1} \epsilon_{-2} \cdots\right)$, the situation will be much more complicated than the above case.

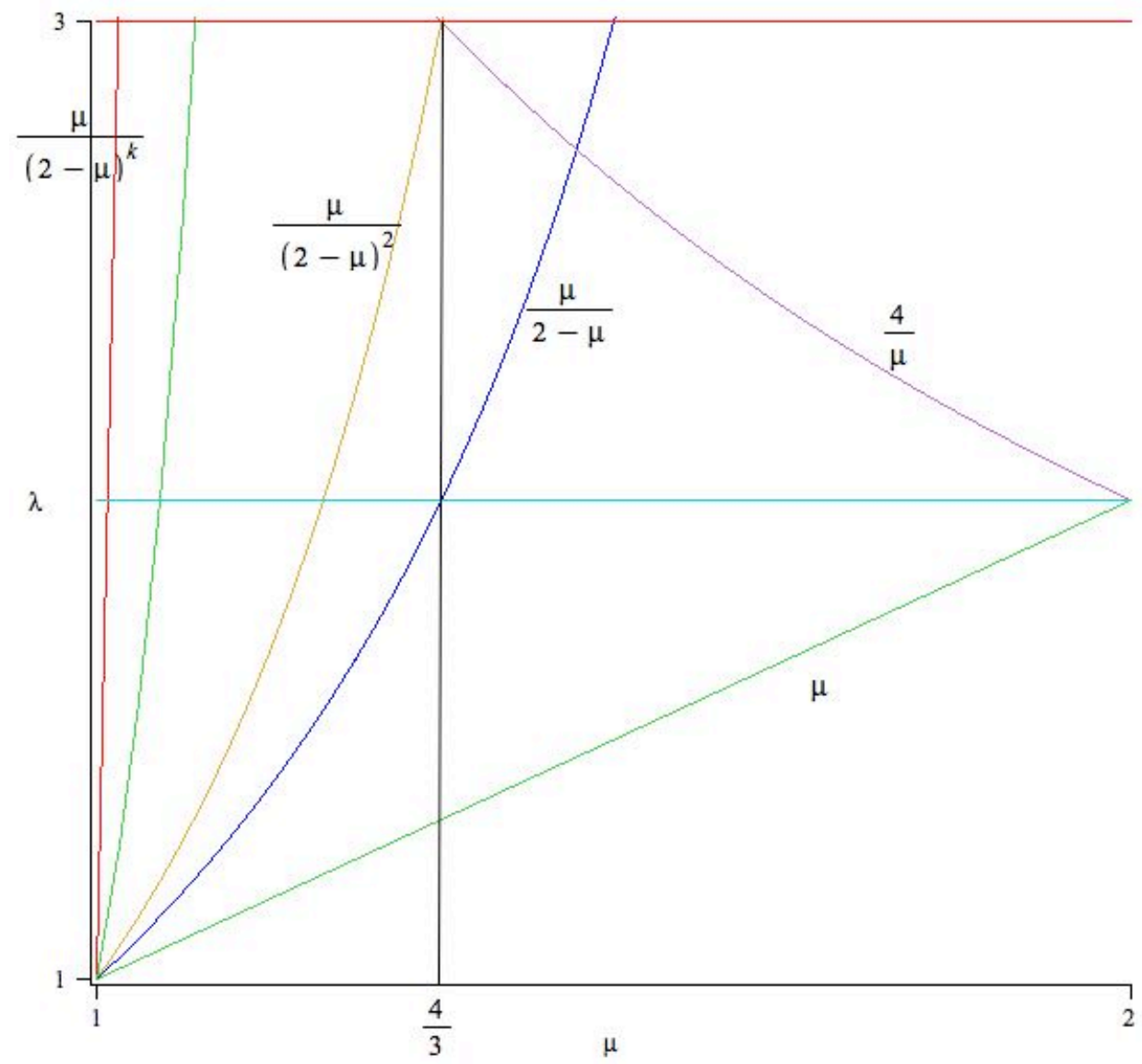

Figure 2.1: The parameter diagram for $1<\mu<\lambda \leqslant 3$

Note $\frac{1}{2}-\frac{1}{2 \mu}<\cdots<\frac{1}{2}-\frac{1}{2 \sqrt[k]{\mu^{k-1} \lambda}}<\frac{1}{2}-\frac{1}{2 \sqrt[k-1]{\mu^{k-2} \lambda}}<\frac{1}{2}-\frac{1}{2 \sqrt{\mu \lambda}}<\frac{1}{2}-\frac{1}{2 \lambda}<1-\frac{1}{\lambda}$ and $f_{\lambda}^{\prime}\left(\frac{1}{2}-\frac{1}{2 \sqrt[k]{\mu^{k-1} \lambda}}\right)\left(f_{\mu}^{\prime}\left(\frac{1}{2}-\frac{1}{2 \sqrt[k]{\mu^{k-1} \lambda}}\right)\right)^{k-1}=1$, for $k \geqslant 1$. So for any $1<\mu<\lambda \leqslant 2$, either $1-\frac{1}{\mu} \in\left(\frac{1}{2}-\frac{1}{2 \lambda}, 1-\frac{1}{\lambda}\right]$ or there exists $k \geqslant 2$ such that $1-\frac{1}{\mu} \in\left(\frac{1}{2}-\frac{1}{2 \sqrt[k]{\mu^{k-1} \lambda}}, \frac{1}{2}-\frac{1}{2 \sqrt[k-1]{\mu^{k-2} \lambda}}\right]$. We consider these cases respectively. They are equivalent to the following cases: $1<\mu<$ $\lambda<\frac{\mu}{2-\mu} ; \frac{\mu}{2-\mu} \leqslant \lambda<\frac{\mu}{(2-\mu)^{2}} ; \cdots ; \frac{\mu}{(2-\mu)^{k-1}} \leqslant \lambda<\frac{\mu}{(2-\mu)^{k}}$ respectively. See Figure 2.1. In the following, we will discuss the general cases. We need a series of lemmas. The proof of these lemmas are quite complicated and technical. We defer them to later sections. 
Lemma 2.1.2 For any $k \geqslant 1,1<\mu<\lambda \leqslant 2$, the following inequality holds,

$$
f_{\mu}\left(\frac{1}{2}-\frac{1}{2 \sqrt[k]{\mu^{k-1} \lambda}}\right)>\frac{1}{2}-\frac{1}{2 \sqrt[k+1]{\mu^{k} \lambda}} .
$$

For any $k \geqslant 2,1<\mu \leqslant 2<\lambda \leqslant 3$, the above inequality (2.1) also holds, while for $k=1$ and $1<\mu \leqslant 2<\lambda \leqslant 3$, we have

$$
f_{\lambda}^{\prime}\left(f_{\mu}\left(\frac{1}{2}-\frac{1}{2 \lambda}\right)\right) f_{\mu}^{\prime}\left(\frac{1}{2}-\frac{1}{2 \lambda}\right)<1 .
$$

Lemma 2.1.3 If $\lambda \geqslant \frac{\mu}{2-\mu}$, then for any $x \in\left[1-\frac{1}{\mu}, \frac{1}{2}-\frac{1}{2 \lambda}\right]$,

$$
f_{\lambda}\left(f_{\mu}(x)\right)>x .
$$

See Figure 2.2.

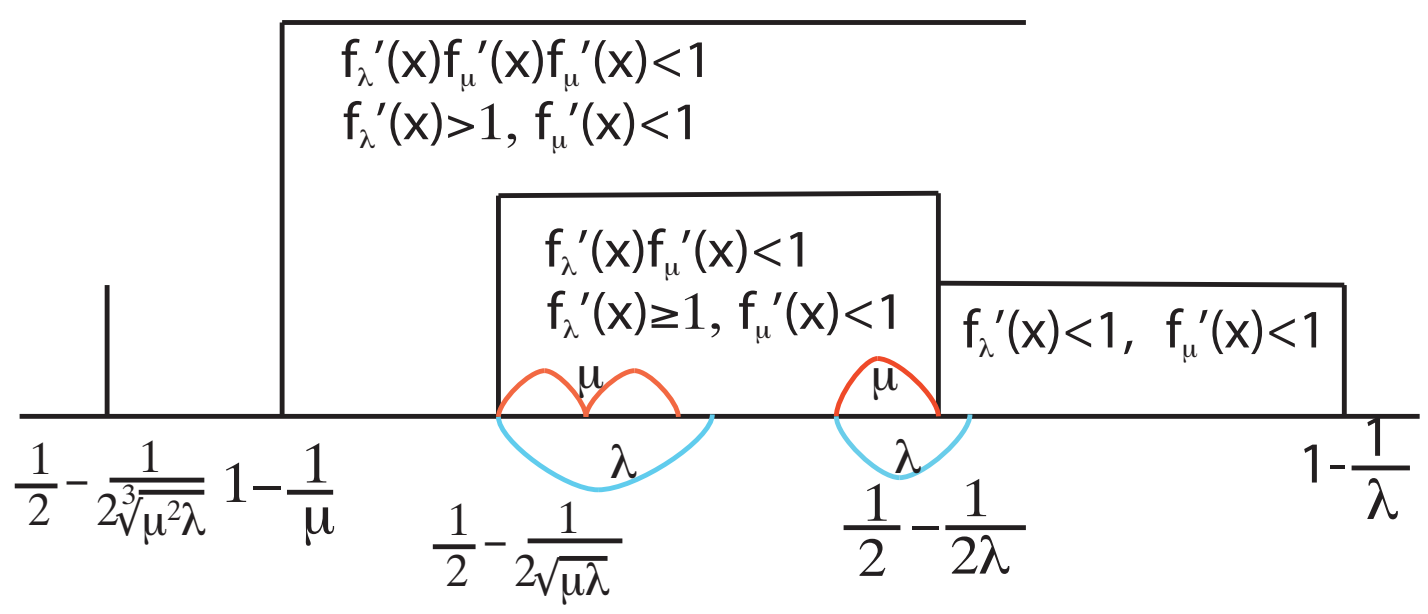

Figure 2.2: $\frac{1}{2}-\frac{1}{2 \sqrt[3]{\mu^{2} \lambda}}<1-\frac{1}{\mu} \leqslant \frac{1}{2}-\frac{1}{2 \sqrt{\mu \lambda}}$

Lemma 2.1.4 Assume $\lambda \geqslant \frac{\mu}{(2-\mu)^{2}}$. Define $a_{2}$ by $f_{\mu}\left(f_{\mu}\left(a_{2}\right)\right)=\frac{1}{2}-\frac{1}{2 \sqrt{\mu \lambda}}$, then for any $x \in\left[1-\frac{1}{\mu}, a_{2}\right]$, we have

$$
f_{\lambda}\left(f_{\mu}\left(f_{\mu}(x)\right)\right)>x
$$

See Figure 2.2. The above result holds in a more general case.

Lemma 2.1.5 For any given $k \geqslant 3$, if $\frac{\mu}{(2-\mu)^{k}} \leqslant \lambda \leqslant 2$, then for any $x \in\left[1-\frac{1}{\mu}, a_{k}\right]$,

$$
f_{\lambda} \underbrace{f_{\mu} \cdots f_{\mu}}_{k}(x)>x
$$

Here $a_{k}$ is defined by $\underbrace{f_{\mu} \cdots f_{\mu}}_{k}\left(a_{k}\right)=\frac{1}{2}-\frac{1}{2 \sqrt[k]{\mu^{k-1} \lambda}}$.

With these lemmas, we can prove proposition 2.1.6. 


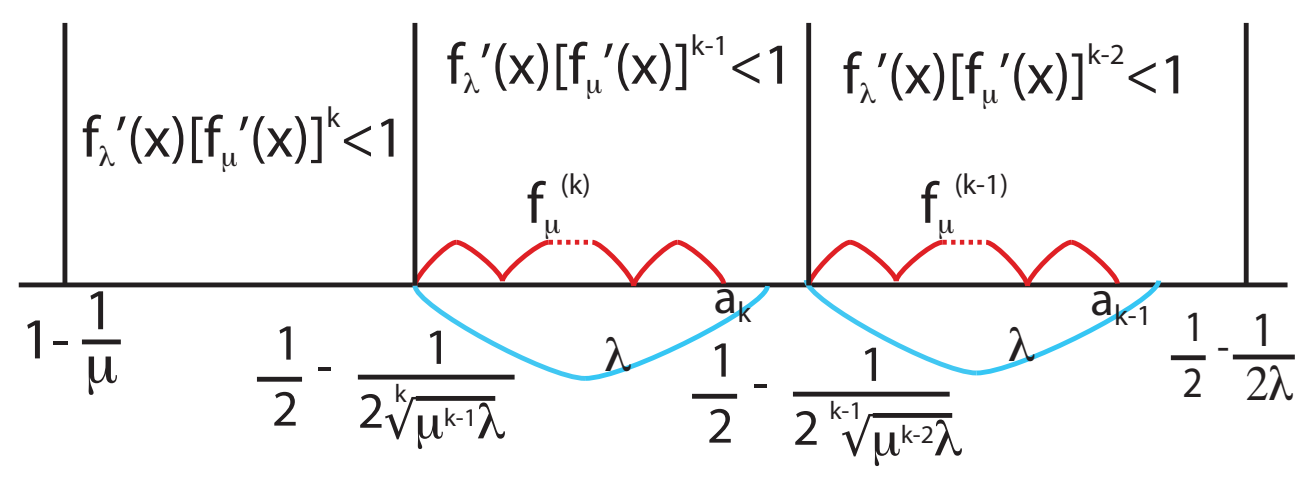

Figure 2.3: $\lambda>\frac{\mu}{(2-\mu)^{k}}$

Proposition 2.1.6 Assume $1<\mu<\lambda<2$. Then for any $x \in\left[1-\frac{1}{\mu}, 1-\frac{1}{\lambda}\right], \lim _{k \rightarrow \infty} f_{\epsilon_{-1}} f_{\epsilon_{-2}} \cdots f_{\epsilon_{-k}}(x)$ exists and the limit is independent of $x$.

Proof. For any $1-\frac{1}{\mu} \leqslant x<y \leqslant 1-\frac{1}{\lambda}$, by mean value theorem, we know that there exists $\xi \in[x, y]$,

$$
\left|f_{\epsilon_{-1}} f_{\epsilon_{-2}} \cdots f_{\epsilon_{-n}}(x)-f_{\epsilon_{-1}} f_{\epsilon_{-2}} \cdots f_{\epsilon_{-n}}(y)\right|=\frac{\partial\left(f_{\epsilon_{-1}} f_{\epsilon_{-2}} \cdots f_{\epsilon_{-n}}(\xi)\right)}{\partial \xi}|x-y| .
$$

The key is to estimate $\left|\frac{\partial\left(f_{\epsilon_{-1}} f_{\epsilon_{-}} \cdots f_{\epsilon_{-n}}(\xi)\right)}{\partial \xi}\right|$ and to prove $\left|\frac{\partial\left(f_{\epsilon_{-1}} f_{\epsilon_{-}} \cdots f_{\epsilon_{-n}}(\xi)\right)}{\partial \xi}\right| \rightarrow 0$ as $n \rightarrow \infty$. We consider different cases.

Case (I). $\xi \in\left[\frac{1}{2}-\frac{1}{2 \lambda}, 1-\frac{1}{\lambda}\right]$. We consider four cases.

1. For any $\xi \in\left[\frac{1}{2}-\frac{1}{2 \lambda}, 1-\frac{1}{\lambda}\right]$, consider $\omega$ such that the process does not leave the interval $\left[\frac{1}{2}-\frac{1}{2 \lambda}, 1-\frac{1}{\lambda}\right]$. Then $f_{\epsilon_{k}}^{\prime} \leqslant 1$ for each $f_{\epsilon_{k}}$ in these maps. However, notice for any $\epsilon_{1}, \epsilon_{2}$, if $f_{\epsilon_{1}}(x) \in\left[\frac{1}{2}-\frac{1}{2 \lambda}, 1-\frac{1}{\lambda}\right]$, then we always have

$$
f_{\epsilon_{2}}^{\prime}\left(f_{\epsilon_{1}}(x)\right) f_{\epsilon_{1}}^{\prime}(x) \leqslant \max \left\{f_{\mu}^{\prime}\left(a_{1}\right) f_{\lambda}^{\prime}\left(\frac{1}{2}-\frac{1}{2 \lambda}\right), f_{\lambda}^{\prime}\left(\frac{1}{2}-\frac{1}{2 \lambda}\right) f_{\lambda}^{\prime}\left(f_{\lambda}\left(\frac{1}{2}-\frac{1}{2 \lambda}\right)\right)\right\}<1,
$$

where $a_{1}$ is defined by $f_{\mu}\left(a_{1}\right)=\frac{1}{2}-\frac{1}{2 \lambda}$. Consequently we can get that

$$
\frac{\partial\left(f_{\epsilon_{-1}} f_{\epsilon_{-2}} \cdots f_{\epsilon_{-n}}(\xi)\right)}{\partial \xi} \leqslant L_{1}^{\left[\frac{n}{2}\right]}
$$

where we choose $L_{1}=\max \left\{f_{\mu}^{\prime}\left(a_{1}\right), f_{\lambda}^{\prime}\left(f_{\lambda}\left(\frac{1}{2}-\frac{1}{2 \lambda}\right)\right)\right\}$.

2. For $\xi \in\left[\frac{1}{2}-\frac{1}{2 \lambda}, 1-\frac{1}{\lambda}\right]$, consider such $\omega$ such that the process is mapped by $f_{\mu}$ to $\left(\frac{1}{2}-\frac{1}{2 \sqrt{\mu \lambda}}, \frac{1}{2}-\frac{1}{2 \lambda}\right)$ at certain time, but never to $\left[1-\frac{1}{\mu}, \frac{1}{2}-\frac{1}{2 \sqrt{\mu \lambda}}\right]$. There are two cases again. The first case is the process will come back to $\left[\frac{1}{2}-\frac{1}{2 \lambda}, 1-\frac{1}{\lambda}\right]$. The second case is to stay in $\left(\frac{1}{2}-\frac{1}{2 \sqrt{\mu \lambda}}, \frac{1}{2}-\frac{1}{2 \lambda}\right)$ forever. 
(a) In the first case, by Lemma 2.1.2 and Lemma 2.1.3, in the sequence from the time it leaves the interval $\left[\frac{1}{2}-\frac{1}{2 \lambda}, 1-\frac{1}{\lambda}\right]$ to the first time it comes back to the interval, it is obvious that the number of $f_{\mu}^{\prime} s$ is more than or equal to the number of $f_{\lambda}^{\prime} s$ as Figure 2.4. But note also any pair of $f_{\mu}$ and $f_{\lambda}$ makes the multiplication of their derivative less than 1 . The largest of these multiplication is

$$
f_{\lambda}^{\prime}\left(\frac{1}{2}-\frac{1}{2 \sqrt{\mu \lambda}}\right) f_{\mu}^{\prime}\left(f_{\mu}\left(a_{2}\right)\right)<1
$$

After that, from the first time it comes back to $\left[\frac{1}{2}-\frac{1}{2 \lambda}, 1-\frac{1}{\lambda}\right]$, the computation of derivative is the same as the case (I) 1 .

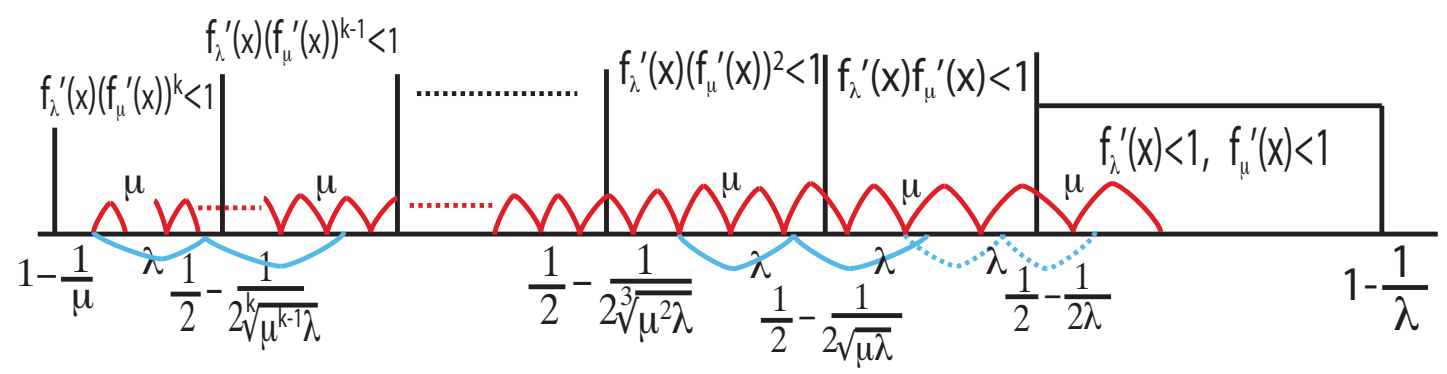

Figure 2.4:

(b) However, if $\xi \in\left[\frac{1}{2}-\frac{1}{2 \lambda}, 1-\frac{1}{\lambda}\right]$ is mapped by $f_{\mu}$ to $\left(\frac{1}{2}-\frac{1}{2 \sqrt{\mu \lambda}}, \frac{1}{2}-\frac{1}{2 \lambda}\right)$ and stays in the interval forever. Then the number of $f_{\mu}^{\prime} s$ is more than the number of $f_{\lambda}^{\prime} s$ in the sequence. As before, any pair of $f_{\mu}$ and $f_{\lambda}$ makes the multiplication of their derivatives less than 1 . The largest it is again

$$
f_{\lambda}^{\prime}\left(\frac{1}{2}-\frac{1}{2 \sqrt{\mu \lambda}}\right) f_{\mu}^{\prime}\left(f_{\mu}\left(a_{2}\right)\right)<1
$$

Therefore, we can see that in case 2 , for any $\xi \in\left[\frac{1}{2}-\frac{1}{2 \lambda}, 1-\frac{1}{\lambda}\right]$,

$$
\frac{\partial\left(f_{\epsilon_{-1}} f_{\epsilon_{-2}} \cdots f_{\epsilon_{-n}}(\xi)\right)}{\partial \xi} \leqslant L_{2}^{\left[\frac{n}{2}\right]}
$$

where $L_{2}=\max \left\{f_{\lambda}^{\prime}\left(\frac{1}{2}-\frac{1}{2 \sqrt{\mu \lambda}}\right) f_{\mu}^{\prime}\left(f_{\mu}\left(a_{2}\right)\right), L_{1}\right\}<1$.

3. For $\xi \in\left[\frac{1}{2}-\frac{1}{2 \lambda}, 1-\frac{1}{\lambda}\right]$, consider the case that the process is mapped by $f_{\mu}$ to $\left(\frac{1}{2}-\frac{1}{2 \sqrt{\mu \lambda}}, \frac{1}{2}-\frac{1}{2 \lambda}\right)$ and then goes to the interval $\left(\frac{1}{2}-\frac{1}{2 \sqrt[3]{\mu^{2} \lambda}}, \frac{1}{2}-\frac{1}{2 \sqrt{\mu \lambda}}\right]$ for certain steps, but never goes to $\left[1-\frac{1}{\mu}, \frac{1}{2}-\frac{1}{2 \sqrt[3]{\mu^{2} \lambda}}\right]$. After that, we consider two subcases.

(a) The process will stay in $\left(\frac{1}{2}-\frac{1}{2 \sqrt[3]{\mu^{2} \lambda}}, \frac{1}{2}-\frac{1}{2 \sqrt{\mu \lambda}}\right]$ forever. Then in the sequence from the time it enters the interval $\left(\frac{1}{2}-\frac{1}{2 \sqrt[3]{\mu^{2} \lambda}}, \frac{1}{2}-\frac{1}{2 \sqrt{\mu \lambda}}\right]$, by Lemma 2.1.4, we know that the number of $f_{\mu}^{\prime} s$ is at least twice as many as the number of $f_{\lambda}^{\prime} s$. So we have

$$
\frac{\partial\left(f_{\epsilon_{-1}} f_{\epsilon_{-2}} \cdots f_{\epsilon_{-n}}(\xi)\right)}{\partial \xi} \leqslant L_{3}^{\left[\frac{n}{3}\right]}
$$


where

$$
L_{3}=\max \left\{f_{\lambda}^{\prime}\left(\frac{1}{2}-\frac{1}{2 \sqrt[3]{\mu^{2} \lambda}}\right)\left(f_{\mu}^{\prime}\left(f_{\mu}\left(f_{\mu}\left(a_{3}\right)\right)\right)\right)^{2}, L_{2}\right\}<1
$$

(b) The process will come back to the interval $\left(\frac{1}{2}-\frac{1}{2 \sqrt{\mu \lambda}}, \frac{1}{2}-\frac{1}{2 \lambda}\right)$. Let $\xi_{1} \in\left(\frac{1}{2}-\right.$ $\left.\frac{1}{2 \sqrt[3]{\mu^{2} \lambda}}, \frac{1}{2}-\frac{1}{2 \sqrt{\mu \lambda}}\right]$ be the point for the first time the process enters $\left(\frac{1}{2}-\frac{1}{2 \sqrt[3]{\mu^{2} \lambda}}, \frac{1}{2}-\right.$ $\left.\frac{1}{2 \sqrt{\mu \lambda}}\right]$. Consider the case which is followed by the map $f_{\lambda}$ that takes the process back to $\left(\frac{1}{2}-\frac{1}{2 \sqrt{\mu \lambda}}, \frac{1}{2}-\frac{1}{2 \lambda}\right)$. Set $\xi_{1}^{\prime}$ by $f_{\mu}\left(f_{\mu}\left(\xi_{1}^{\prime}\right)\right)=\xi_{1}$. Since the start point $\xi \in\left[\frac{1}{2}-\frac{1}{2 \lambda}, 1-\frac{1}{\lambda}\right]$ and by Lemma 2.1.2, $\xi_{1}^{\prime}<1-\frac{1}{\lambda}$ and $f_{\mu}\left(\xi_{1}^{\prime}\right)>\frac{1}{2}-\frac{1}{2 \sqrt{\mu \lambda}}$. In this sequence, from the time that the process at $\xi_{1}^{\prime}$ to the time it leaves that interval and reaches $f_{\lambda}\left(\xi_{1}\right)>\xi_{1}^{\prime}$, there are two $f_{\mu}^{\prime} s$ and one $f_{\lambda}^{\prime} s$.

While if after reaching $\xi_{1}$, the process does not leave the interval immediately but later. There are two cases. From the time at $\xi_{1}$ to the time the process back to the interval $\left(\frac{1}{2}-\frac{1}{2 \sqrt{\mu \lambda}}, \frac{1}{2}-\frac{1}{2 \lambda}\right)$, if there are $2 l(l \in N) f_{\mu}^{\prime} s$, then from the time the process at $\xi_{1}^{\prime}$ to the first time it goes back to $\left(\frac{1}{2}-\frac{1}{2 \sqrt{\mu \lambda}}, \frac{1}{2}-\frac{1}{2 \lambda}\right)$, the number of $f_{\mu}^{\prime} s$ is at least twice as many as the number of $f_{\lambda}^{\prime} s$. Otherwise, if there are $(2 l+1) f_{\mu}$, then from the time the process at $f_{\mu}\left(\xi_{1}^{\prime}\right)$ to the first time it goes back to $\left(\frac{1}{2}-\frac{1}{2 \sqrt{\mu \lambda}}, \frac{1}{2}-\frac{1}{2 \lambda}\right)$, the number of $f_{\mu}^{\prime} s$ is at least twice as many as the number of $f_{\lambda}^{\prime} s$.

In other words, we can also have (2.8) in any case of $(\mathrm{I})(3)(\mathrm{b})$.

4. Consider the case that the point from $\left[\frac{1}{2}-\frac{1}{2 \lambda}, 1-\frac{1}{\lambda}\right]$ continue to be mapped into the interval $\left[1-\frac{1}{\mu}, \frac{1}{2}-\frac{1}{2 \sqrt[k]{\mu^{k-1} \lambda}}\right]$ after it enters the interval $\left(\frac{1}{2}-\frac{1}{2 \sqrt[k]{\mu^{k-1} \lambda}}, \frac{1}{2}-\frac{1}{2 \sqrt[k-1]{\mu^{k-2} \lambda}}\right]$. Note in this interval $\left[1-\frac{1}{\mu}, \frac{1}{2}-\frac{1}{2 \sqrt[k]{\mu^{k-1} \lambda}}\right]$,

$$
f_{\lambda}^{\prime}(x)\left[f_{\mu}^{\prime}(x)\right]^{k}<1 .
$$

There are two possibilities.

(a) The process will remain in $\left[1-\frac{1}{\mu}, \frac{1}{2}-\frac{1}{2 \sqrt[k]{\mu^{k-1} \lambda}}\right]$ forever. Then by Lemma 2.1.2 and Lemma 2.1.5, in the sequence, from the first time the process enters the interval $\left[1-\frac{1}{\mu}, \frac{1}{2}-\frac{1}{2 \sqrt[k]{\mu^{k-1} \lambda}}\right]$, the number of $f_{\mu}^{\prime} s$ is at least $k$ times as many as the number of $f_{\lambda}^{\prime} s$. Again any $k f_{\mu}^{\prime} s$ and one $f_{\lambda}$ make the multiplication of their derivatives less than 1 , the largest of these is

$$
f_{\lambda}^{\prime}\left(1-\frac{1}{\mu}\right)\left(f_{\mu}^{\prime}\left(1-\frac{1}{\mu}\right)\right)^{k}<1
$$

Hence we can get

$$
\frac{\partial\left(f_{\epsilon_{-1}} f_{\epsilon_{-2}} \cdots f_{\epsilon_{-n}}(\xi)\right)}{\partial \xi} \leqslant L_{k+1}^{\left[\frac{n}{k+1}\right]}
$$

where

$$
L_{k+1}=\max \left\{f_{\lambda}^{\prime}\left(1-\frac{1}{\mu}\right)\left(f_{\mu}^{\prime}\left(1-\frac{1}{\mu}\right)\right)^{k}, L_{k}\right\}<1,
$$




$$
\text { and } L_{k}=\max \left\{f_{\lambda}^{\prime}\left(\frac{1}{2}-\frac{1}{2 \sqrt[k]{\mu^{k-1} \lambda}}\right)\left[f_{\mu}^{\prime}(\underbrace{f_{\mu} \cdots f_{\mu}}_{k-1}\left(a_{k}\right))\right]^{k-1}, L_{k-1}\right\} \text {. }
$$

(b) The process will leave $\left[1-\frac{1}{\mu}, \frac{1}{2}-\frac{1}{2 \sqrt[k]{\mu^{k-1} \lambda}}\right]$, to $\left(\frac{1}{2}-\frac{1}{2 \sqrt[k]{\mu^{k-1} \lambda}}, \frac{1}{2}-\frac{1}{2 \sqrt[k-1]{\mu^{k-2} \lambda}}\right]$ and assume it stays in it forever. Then by Lemma 2.1.5, the number of $f_{\mu}^{\prime} s$ is at least $k-1$ times as many as the number of $f_{\lambda}^{\prime} s$. Again any $k-1 f_{\mu}^{\prime} s$ and one $f_{\lambda}$ make the multiplication of their derivatives less than 1 , the largest of these is

$$
f_{\lambda}^{\prime}\left(\frac{1}{2}-\frac{1}{2 \sqrt[k]{\mu^{k-1} \lambda}}\right)\left[f_{\mu}^{\prime}(\underbrace{f_{\mu} \cdots f_{\mu}}_{k-1}\left(a_{k}\right))\right]^{k-1}<1 .
$$

So we have

$$
\frac{\partial\left(f_{\epsilon_{-1}} f_{\epsilon_{-2}} \cdots f_{\epsilon_{-n}}(\xi)\right)}{\partial \xi} \leqslant L_{k}^{\left[\frac{n}{k}\right]}
$$

where $L_{k}=\max \left\{f_{\lambda}^{\prime}\left(\frac{1}{2}-\frac{1}{2 \sqrt[k]{\mu^{k-1} \lambda}}\right)\left[f_{\mu}^{\prime}(\underbrace{f_{\mu} \cdots f_{\mu}}_{k-1}\left(a_{k}\right))\right]^{k-1}, L_{k-1}\right\}$.

(c) In case (b), if the point will not stay in $\left(\frac{1}{2}-\frac{1}{2 \sqrt[k]{\mu^{k-1} \lambda}}, \frac{1}{2}-\frac{1}{2 \sqrt[k-1]{\mu^{k-2} \lambda}}\right]$ forever and it is mapped into $\left[1-\frac{1}{\mu}, \frac{1}{2}-\frac{1}{2 \sqrt[k]{\mu^{k-1} \lambda}}\right]$ again. After that it can be case (a) or the point will be mapped into $\left(\frac{1}{2}-\frac{1}{2 \sqrt[k]{\mu^{k-1} \lambda}}, \frac{1}{2}-\frac{1}{2 \sqrt[k-1]{\mu^{k-2} \lambda}}\right]$ again. Let $\xi_{1} \in\left[1-\frac{1}{\mu}, \frac{1}{2}-\frac{1}{2 \sqrt[k]{\mu^{k-1} \lambda}}\right]$ be the point for the first time the process enters $\left[1-\frac{1}{\mu}, \frac{1}{2}-\frac{1}{2 \sqrt[k]{\mu^{k-1} \lambda}}\right]$. Consider the case above is immediately followed by the map $f_{\lambda}$ that takes the process back to $\left(\frac{1}{2}-\frac{1}{2 \sqrt[k]{\mu^{k-1} \lambda}}, \frac{1}{2}-\frac{1}{2 \sqrt[k-1]{\mu^{k-2} \lambda}}\right]$. Set $\xi_{1}^{\prime}$ by $f_{\mu}(\underbrace{f_{\mu} \cdots f_{\mu}}_{k-1}\left(\xi_{1}^{\prime}\right))=\xi_{1}$. Since the starting point $\xi \in\left[\frac{1}{2}-\frac{1}{2 \lambda}, 1-\frac{1}{\lambda}\right]$, so by Lemma $2.1 .2, \xi_{1}^{\prime}<1-\frac{1}{\lambda}$. In this sequence, from the time that the process at $\xi_{1}^{\prime}$ to the time it leaves that interval and reaches $f_{\lambda}\left(\xi_{1}\right)>\xi_{1}^{\prime}$, there are $k f_{\mu}^{\prime} s$ and one $f_{\lambda}^{\prime} s$.

While if after the process reaches $\xi_{1}$, it does not leave the interval immediately but later. Let $\xi_{i} \in\left[1-\frac{1}{\mu}, \frac{1}{2}-\frac{1}{2 \sqrt[k]{\mu^{k-1} \lambda}}\right], \xi_{i}=f_{\mu}\left(\xi_{i-1}\right), i=2, \cdots, k$. It is easy to see that $\xi_{i}>\underbrace{f_{\mu} \cdots f_{\mu}}_{i}\left(\frac{1}{2}-\frac{1}{2 \sqrt[k]{\mu^{k-1} \lambda}}\right)$ and $\xi_{k}<\xi_{k-1}<\cdots<\xi_{i}<\cdots<\xi_{2}<\xi_{1}$.

We set that $\xi_{i}$ is the point which starts to move to the right and into the interval $\left(\frac{1}{2}-\frac{1}{2 \sqrt[k]{\mu^{k-1} \lambda}}, \frac{1}{2}-\frac{1}{2 \sqrt[k-1]{\mu^{k-2} \lambda}}\right]$ by $f_{\lambda}$. Set $\xi_{i}^{\prime}$ by $f_{\mu}(\underbrace{f_{\mu} \cdots f_{\mu}}_{k-1}\left(\xi_{i}^{\prime}\right))=\xi_{i}$ and obviously, $\xi_{i}^{\prime}>\frac{1}{2}-\frac{1}{2 \sqrt[k]{\mu^{k-1} \lambda}}$ and $f_{\mu}\left(\xi_{k}^{\prime}\right)=\xi_{1}$ as Figure 2.5 shows. From the time that the process at $\xi_{i}^{\prime}$ to the time it leaves that interval and reaches $f_{\lambda}\left(\xi_{i}\right)>\xi_{i}^{\prime}$, there are $k f_{\mu}^{\prime} s$ and one $f_{\lambda}^{\prime} s$.

Furthermore, if after the process reaches $\xi_{k}$, but it does not leave the interval immediately but later. With the same reason as $(\mathrm{I})(3)(\mathrm{b})$, there are $k$ cases. 


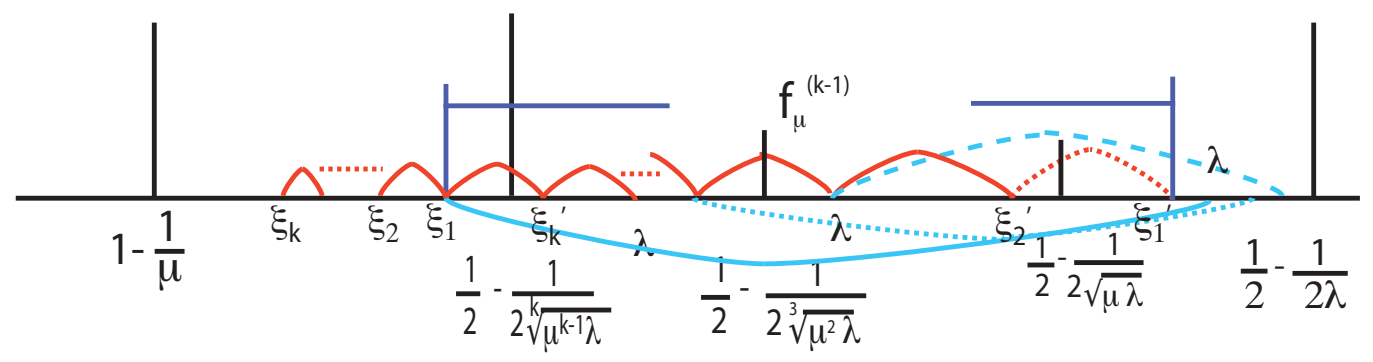

Figure 2.5: $\xi_{1}^{\prime}<\frac{1}{2}-\frac{1}{2 \lambda}$

Generally speaking, we will describe the $i-t h$ case as follows. From the time at $\xi_{1}$ to the time the process back to the interval $\left(\frac{1}{2}-\frac{1}{2 \sqrt[k]{\mu^{k-1} \lambda}}, \frac{1}{2}-\frac{1}{2 \sqrt[k-1]{\mu^{k-2} \lambda}}\right]$, if there are $l k+i(l \in N, i \in N) f_{\mu}$. From the time the process at $\xi_{i}^{\prime}$ to the first time it goes back to $\left(\frac{1}{2}-\frac{1}{2 \sqrt[k]{\mu^{k-1} \lambda}}, \frac{1}{2}-\frac{1}{2 \sqrt[k-1]{\mu^{k-2} \lambda}}\right]$, the number of $f_{\mu}^{\prime} s$ is at least $k$ times as many as the number of $f_{\lambda}^{\prime} s$. So we can also get (2.9) as well.

After that, it will be the previous cases.

Summarizing above, we can get for any $\xi \in\left[\frac{1}{2}-\frac{1}{2 \lambda}, 1-\frac{1}{\lambda}\right]$,

$$
\frac{\partial\left(f_{\epsilon_{-1}} f_{\epsilon_{-2}} \cdots f_{\epsilon_{-n}}(\xi)\right)}{\partial \xi} \leqslant L_{k+1}^{\left[\frac{n}{k+1}\right]} .
$$

Case (II). $\xi \in\left(\frac{1}{2}-\frac{1}{2 \sqrt{\mu \lambda}}, \frac{1}{2}-\frac{1}{2 \lambda}\right)$.

1. If for any $\xi \in\left(\frac{1}{2}-\frac{1}{2 \sqrt{\mu \lambda}}, \frac{1}{2}-\frac{1}{2 \lambda}\right)$, the process can enter the interval $\left[\frac{1}{2}-\frac{1}{2 \lambda}, 1-\frac{1}{\lambda}\right]$ as Figure 2.5 shows. Consider that $f_{\lambda}^{\prime}(\xi)>1$ and $f_{\lambda}^{\prime}(\xi) f_{\mu}\left({ }^{\prime} \xi\right)<1$ for $\xi \in\left(\frac{1}{2}-\frac{1}{2 \sqrt{\mu \lambda}}, \frac{1}{2}-\right.$ $\left.\frac{1}{2 \lambda}\right)$. It is easy to see that there exists $n_{1}$ such that when $f_{\lambda}\left(f_{\lambda} \cdots f_{\lambda}\left(\frac{1}{2}-\frac{1}{2 \sqrt{\mu \lambda}}\right)\right)>$ $\frac{1}{2}-\frac{1}{2 \lambda}$, where $n_{1}$ is the minimum number of $f_{\lambda}$ in this sequence. From time 0 till the first time that it enters $\left[\frac{1}{2}-\frac{1}{2 \lambda}, 1-\frac{1}{\lambda}\right]$, there are at most $n_{1}$ more $f_{\lambda}^{\prime} s$ than the number of $f_{\mu}^{\prime} s$. After that, it will be case (I). By Lemma 2.1.3, then for any $\xi \in\left(\frac{1}{2}-\frac{1}{2 \sqrt{\mu \lambda}}, \frac{1}{2}-\frac{1}{2 \lambda}\right)$, we have

$$
\frac{\partial\left(f_{\epsilon_{-1}} f_{\epsilon_{-2}} \cdots f_{\epsilon_{-n}}(\xi)\right)}{\partial \xi} \leqslant M_{1}^{m_{1}} L_{k+1}^{\left[\frac{n-m_{1}}{k+1}\right]}
$$

here $M_{1}=f_{\lambda}^{\prime}\left(\frac{1}{2}-\frac{1}{2 \sqrt{\mu \lambda}}\right)$ and $m_{1}=n_{1}$.

2. If $\xi \in\left(\frac{1}{2}-\frac{1}{2 \sqrt{\mu \lambda}}, \frac{1}{2}-\frac{1}{2 \lambda}\right)$, the process stays in this interval forever, then for any $\xi \in\left(\frac{1}{2}-\frac{1}{2 \sqrt{\mu \lambda}}, \frac{1}{2}-\frac{1}{2 \lambda}\right)$, it will be same as case $(\mathrm{I})(2)(\mathrm{b})$. 
3. If $\xi \in\left(\frac{1}{2}-\frac{1}{2 \sqrt{\mu \lambda}}, \frac{1}{2}-\frac{1}{2 \lambda}\right)$ is mapped into $\left(\frac{1}{2}-\frac{1}{2 \sqrt[3]{\mu^{2} \lambda}}, \frac{1}{2}-\frac{1}{2 \sqrt{\mu \lambda}}\right]$ and never to $[1-$ $\left.\frac{1}{\mu}, \frac{1}{2}-\frac{1}{2 \sqrt[3]{\mu^{2} \lambda}}\right]$

(a) After that, if the process stays in $\left(\frac{1}{2}-\frac{1}{2 \sqrt[3]{\mu^{2} \lambda}}, \frac{1}{2}-\frac{1}{2 \sqrt{\mu \lambda}}\right]$ forever, we have discussed this case in case (I)(3)(a).

(b) However if the process can go back to $\left(\frac{1}{2}-\frac{1}{2 \sqrt{\mu \lambda}}, \frac{1}{2}-\frac{1}{2 \lambda}\right)$ as Figure 2.6 shows. Assume the process enters $\left(\frac{1}{2}-\frac{1}{2 \sqrt[3]{\mu^{2} \lambda}}, \frac{1}{2}-\frac{1}{2 \sqrt{\mu \lambda}}\right]$ at the first time from a point $d_{2} \in\left(\frac{1}{2}-\frac{1}{2 \sqrt{\mu \lambda}}, \frac{1}{2}-\frac{1}{2 \lambda}\right)$. Consider the case that the process goes back to $\left(\frac{1}{2}-\frac{1}{2 \sqrt{\mu \lambda}}, \frac{1}{2}-\frac{1}{2 \lambda}\right)$ immediately as Figure 2.6 shows. As $d_{2} \in\left(f_{\mu}\left(a_{2}\right), \frac{1}{2}-\frac{1}{2 \sqrt{\mu \lambda}}\right)$, then by Lemma 2.1.4, we have

$$
d_{2}^{\prime}:=f_{\lambda}\left(f_{\mu}\left(d_{2}\right)\right)>f_{\lambda}\left(\frac{1}{2}-\frac{1}{2 \sqrt{\mu \lambda}}\right), \quad f_{\mu}\left(d_{2}^{\prime}\right)>d_{2}>\frac{1}{2}-\frac{1}{2 \sqrt{\mu \lambda}} .
$$

After that, if $d_{2}^{\prime}$ keeps in the interval $\left(\frac{1}{2}-\frac{1}{2 \sqrt{\mu \lambda}}, \frac{1}{2}-\frac{1}{2 \lambda}\right)$, that will be case 2. However if case (3)(b) happens again, then by (2.13), Lemma 2.1.4 and Lemma 2.1.2, from the time at $d_{2}^{\prime}$ to the time that the process comes back to $\left(\frac{1}{2}-\frac{1}{2 \sqrt{\mu \lambda}}, \frac{1}{2}-\frac{1}{2 \lambda}\right)$, the number of $f_{\mu}^{\prime} s$ is at least twice as many as the number of $f_{\lambda}^{\prime} s$. In this case, we can get the largest derivative of this multiplication as follows,

$$
f_{\lambda}^{\prime}\left(f_{\mu}\left(d_{2}\right)\right) f_{\mu}^{\prime}\left(d_{2}\right)<\max \left\{f_{\lambda}^{\prime}\left(f_{\mu}\left(\frac{1}{2}-\frac{1}{2 \sqrt{\mu \lambda}}\right)\right) f_{\mu}^{\prime}\left(\frac{1}{2}-\frac{1}{2 \sqrt{\mu \lambda}}\right)\right\}:=N_{2} .
$$

Obviously, $N_{2}>1$. After that it will be case 2 again or repeat case 3 . In this

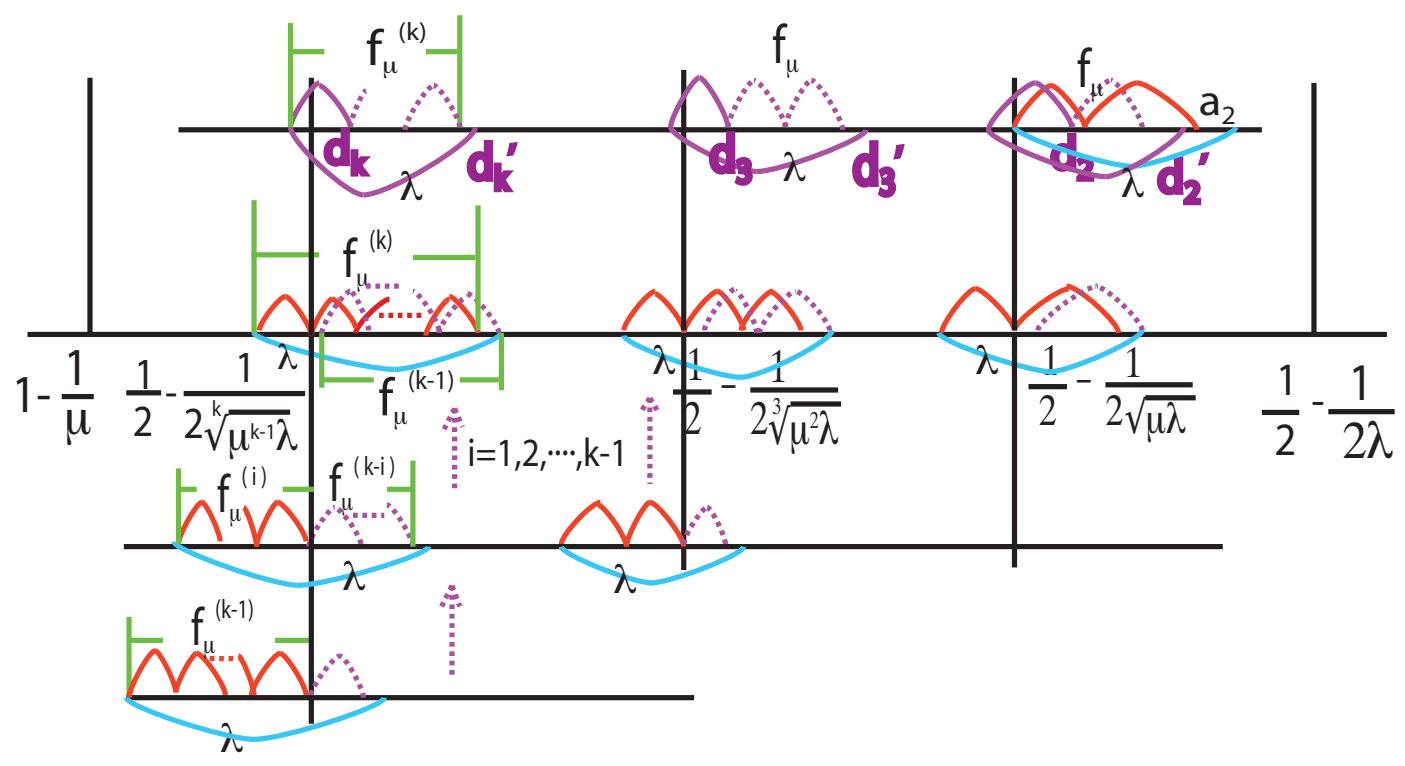

Figure 2.6: case (b) 
case, one can easily obtain the following inequality:

$$
\frac{\partial\left(f_{\epsilon_{-1}} f_{\epsilon_{-2}} \cdots f_{\epsilon_{-n}}(\xi)\right)}{\partial \xi} \leqslant N_{2} L_{3}^{\left[\frac{n-2}{3}\right]} .
$$

4. Consider the case that if $\xi \in\left(\frac{1}{2}-\frac{1}{2 \sqrt{\mu \lambda}}, \frac{1}{2}-\frac{1}{2 \lambda}\right)$ is mapped into the interval [1$\left.\frac{1}{\mu}, \frac{1}{2}-\frac{1}{2 \sqrt[k]{\mu^{k-1} \lambda}}\right]$ after the process enters $\left(\frac{1}{2}-\frac{1}{2 \sqrt[k]{\mu^{k-1} \lambda}}, \frac{1}{2}-\frac{1}{2 \sqrt[k-1]{\mu^{k-2} \lambda}}\right]$.

(a) After that, if the process stays in $\left[1-\frac{1}{\mu}, \frac{1}{2}-\frac{1}{2 \sqrt[k]{\mu^{k-1} \lambda}}\right]$ forever, that is case $(\mathrm{I})(4)(\mathrm{a})$. However, if the process goes back to $\left(\frac{1}{2}-\frac{1}{2 \sqrt[k]{\mu^{k-1} \lambda}}, \frac{1}{2}-\frac{1}{2 \sqrt[k-1]{\mu^{k-2} \lambda}}\right]$ and stays in this interval forever, it is case (I)(4)(b).

(b) Otherwise, if the process is mapped back to the interval $\left(\frac{1}{2}-\frac{1}{2 \sqrt[k]{\mu^{k-1} \lambda}}, \frac{1}{2}-\right.$ $\left.\frac{1}{2 \sqrt[k-1]{\mu^{k-2} \lambda}}\right]$ again after it enters $\left[1-\frac{1}{\mu}, \frac{1}{2}-\frac{1}{2 \sqrt[k]{\mu^{k-1} \lambda}}\right]$. Let $\xi_{1} \in\left[1-\frac{1}{\mu}, \frac{1}{2}-\right.$ $\left.\frac{1}{2 \sqrt[k]{\mu^{k-1} \lambda}}\right]$ be the point for the first time the process enters $\left[1-\frac{1}{\mu}, \frac{1}{2}-\frac{1}{2 \sqrt[k]{\mu^{k-1} \lambda}}\right]$. Consider the case that above process is immediately followed by the map $f_{\lambda}$ that takes the process out of $\left(\frac{1}{2}-\frac{1}{2 \sqrt[k]{\mu^{k-1} \lambda}}, \frac{1}{2}-\frac{1}{2 \sqrt[k-1]{\mu^{k-2} \lambda}}\right]$. Set $\xi_{1}^{\prime}$ by $(\underbrace{f_{\mu} \cdots f_{\mu}}_{k}\left(\xi_{1}^{\prime}\right))=$ $\xi_{1}$. So by Lemma 2.1.2, $f_{\mu}\left(\xi_{1}^{\prime}\right)<\frac{1}{2}-\frac{1}{2 \lambda}$. Then in this sequence, from the time that the process at $f_{\mu}\left(\xi_{1}^{\prime}\right)$ to the time it leaves that interval and reaches $f_{\lambda}\left(\xi_{1}\right)>\xi_{1}^{\prime}$, there are $k-1 f_{\mu}^{\prime} s$ and one $f_{\lambda}^{\prime} s$ as Figure 2.5 shows. After that, if this process is repeated, from the time that the process at $f_{\lambda}\left(\xi_{1}\right)$ to the time it leaves that interval and reaches $f_{\lambda}(\underbrace{f_{\mu} \cdots f_{\mu}}_{k}\left(f_{\lambda}\left(\xi_{1}\right)\right))>f_{\lambda}\left(\xi_{1}\right)$, there are $k f_{\mu}^{\prime} s$ and one $f_{\lambda}^{\prime} s$. The largest derivative of this multiplication is $\frac{d}{d x}[f_{\lambda} \underbrace{f_{\mu} \cdots f_{\mu}}_{k-1}\left(\frac{1}{2}-\right.$ $\left.\left.\frac{1}{2 \sqrt[k]{\mu^{k-1} \lambda}}\right)\right]:=N_{k k}$

On the other hand, if we have $\xi_{1}^{\prime}<\frac{1}{2}-\frac{1}{2 \lambda}$, then in this sequence, from the time that the process at $\xi_{1}^{\prime}$ to the time it leaves that interval and reaches $f_{\lambda}\left(\xi_{1}\right)>\xi_{1}^{\prime}$, there are $k f_{\mu}^{\prime} s$ and one $f_{\lambda}^{\prime} s$,

$$
\frac{\partial\left(f_{\epsilon_{-1}} f_{\epsilon_{-2}} \cdots f_{\epsilon_{-n}}(\xi)\right)}{\partial \xi} \leqslant N_{k k} L_{k+1}^{\left[\frac{n-k}{k+1}\right]}
$$

In fact, if the above process is immediately followed by case (II)(3)(b), then we can let $\xi_{1} \in\left(\frac{1}{2}-\frac{1}{2 \sqrt[i]{\mu^{i-1} \lambda}}, \frac{1}{2}-\frac{1}{2 \sqrt[i-1]{\mu^{i-2} \lambda}}\right](i=3, \cdots, k+1)$ be the point for the first time the process enters $\left(\frac{1}{2}-\frac{1}{2 \sqrt[i]{\mu^{i-1} \lambda}}, \frac{1}{2}-\frac{1}{2 \sqrt[i-1]{\mu^{i-2} \lambda}}\right]$, then we consider the case above is immediately followed by the map $f_{\lambda}$ that takes the process out of $\left(\frac{1}{2}-\frac{1}{2 \sqrt[i]{\mu^{i-1} \lambda}}, \frac{1}{2}-\frac{1}{2 \sqrt[i-1]{\mu^{i-2} \lambda}}\right]$. Set $\xi_{1}^{\prime}$ by $f_{\mu}(\underbrace{f_{\mu} \cdots f_{\mu}}_{i}\left(\xi_{1}^{\prime}\right))=\xi_{1}$. Since the starting point $\xi \in\left(\frac{1}{2}-\frac{1}{2 \sqrt{\mu \lambda}}, \frac{1}{2}-\frac{1}{2 \lambda}\right)$, so by Lemma 2.1.2, $f_{\mu}\left(\xi_{1}^{\prime}\right)<\frac{1}{2}-\frac{1}{2 \lambda}$. Therefore, in this sequence, from the time that the process at $f_{\mu}\left(\xi_{1}^{\prime}\right)$ to the time it leaves that interval and reaches $f_{\lambda}\left(\xi_{1}\right)>\xi_{1}^{\prime}$, there are $i-1 f_{\mu}^{\prime} s$ and 
one $f_{\lambda}^{\prime} s$ with the same reason as above. After that, if this process repeated, from the time that the process at $f_{\lambda}\left(\xi_{1}\right)$ to the time it leaves that interval and reaches $f_{\lambda}(\underbrace{f_{\mu} \cdots f_{\mu}}_{i}\left(f_{\lambda}\left(\xi_{1}\right)\right))>f_{\lambda}\left(\xi_{1}\right)$, there are $i f_{\mu}^{\prime} s$ and one $f_{\lambda}^{\prime} s$. The largest derivative of this multiplication is $\frac{d}{d x}[f_{\lambda} \underbrace{f_{\mu} \cdots f_{\mu}}_{i-1}\left(\frac{1}{2}-\frac{1}{2 \sqrt[i]{\mu^{i-1} \lambda}}\right)]:=N_{i i}$. If $\xi_{1} \in\left[1-\frac{1}{\mu}, \frac{1}{2}-\frac{1}{2 \sqrt[k]{\mu^{k-1} \lambda}}\right]$, it will be case (II)(4)(b). Obviously, $N_{k k}=\max \left\{N_{i i}\right\}$.

Concluding the above four cases, for any $\xi \in\left(\frac{1}{2}-\frac{1}{2 \sqrt{\mu \lambda}}, \frac{1}{2}-\frac{1}{2 \lambda}\right)$, we have

$$
\frac{\partial\left(f_{\epsilon_{-1}} f_{\epsilon_{-2}} \cdots f_{\epsilon_{-n}}(\xi)\right)}{\partial \xi} \leqslant M_{1}^{m_{1}} N_{k k} L_{k+1}^{\left[\frac{n-m_{1}-k}{k+1}\right]} .
$$

Case (III). $\xi \in\left(\frac{1}{2}-\frac{1}{2 \sqrt[k]{\mu^{k-1} \lambda}}, \frac{1}{2}-\frac{1}{2 \sqrt[k-1]{\mu^{k-2} \lambda}}\right]$.

1. If $\omega$ makes the process stay in $\left(\frac{1}{2}-\frac{1}{2 \sqrt[k]{\mu^{k-1} \lambda}}, \frac{1}{2}-\frac{1}{2 \sqrt[k-1]{\mu^{k-2} \lambda}}\right]$ forever, then it is similar to case $(\mathrm{I})(4)(\mathrm{b})$.

2. If the process enters $\left[1-\frac{1}{\mu}, \frac{1}{2}-\frac{1}{2 \sqrt[k]{\mu^{k-1} \lambda}}\right]$ and stays in it forever, then it is the case (I) $(4)(\mathrm{a})$.

3. If $\xi \in\left(\frac{1}{2}-\frac{1}{2 \sqrt[k]{\mu^{k-1} \lambda}}, \frac{1}{2}-\frac{1}{2 \sqrt[k-1]{\mu^{k-2} \lambda}}\right]$ is mapped into $\left[1-\frac{1}{\mu}, \frac{1}{2}-\frac{1}{2 \sqrt[k]{\mu^{k-1} \lambda}}\right]$ and then enters $\left(\frac{1}{2}-\frac{1}{2 \sqrt[k]{\mu^{k-1} \lambda}}, \frac{1}{2}-\frac{1}{2 \sqrt[k-1]{\mu^{k-2} \lambda}}\right]$ again by the map $f_{\lambda}$ at some stage, then from the first time the process leaves the interval $\left(\frac{1}{2}-\frac{1}{2 \sqrt[k]{\mu^{k-1} \lambda}}, \frac{1}{2}-\frac{1}{2 \sqrt[k-1]{\mu^{k-2} \lambda}}\right]$ to the first time the process enters $\left(\frac{1}{2}-\frac{1}{2 \sqrt[k]{\mu^{k-1} \lambda}}, \frac{1}{2}-\frac{1}{2 \sqrt[k-1]{\mu^{k-2} \lambda}}\right]$ again, with the same reason as case (II)(3)(b), by Lemma 2.1.5, see Figure 2.6, it is easy to see the last point $d_{k}$ at which the process leaves $\left(\frac{1}{2}-\frac{1}{2 \sqrt[k]{\mu^{k-1} \lambda}}, \frac{1}{2}-\frac{1}{2 \sqrt[k-1]{\mu^{k-2} \lambda}}\right]$ should satisfy $d_{k} \in(\underbrace{f_{\mu} \cdots f_{\mu}}_{k-1}\left(a_{k}\right), \frac{1}{2}-\frac{1}{2 \sqrt[k]{\mu^{k-1} \lambda}})$. From Figure 2.6, since $f_{\lambda}^{\prime}(\tilde{x})\left(f_{\mu}^{\prime}(\tilde{x})\right)^{i-1}>1$ for any $\tilde{x} \in\left[1-\frac{1}{\mu}, \frac{1}{2}-\frac{1}{2 \sqrt[k]{\mu^{k-1} \lambda}}\right]$ and $i=2, \cdots, k$, then for the process like (III)(3). It is easy to see that the largest derivative of the multiplication can be computed as follows.

So for any integer $i \in[2, k]$, we have

$$
\frac{d}{d x}\left[f_{\lambda}(\underbrace{f_{\mu} \cdots f_{\mu}}_{i-1}\left(d_{k}\right))\right]<\max \left\{\frac{d}{d x}\left[f_{\lambda}(\underbrace{f_{\mu} \cdots f_{\mu}}_{i-1}\left(\frac{1}{2}-\frac{1}{2 \sqrt[k]{\mu^{k-1} \lambda}}\right))\right]\right\}=N_{k i} .
$$

Obviously $N_{k i}>1$ is possible. For the process $f_{\lambda}(\underbrace{f_{\mu} \cdots f_{\mu}}_{i-1}\left(d_{k}\right))$, there are $i$ steps and

$$
\sum_{i=2}^{k}(i)=\frac{(k-1)(k+2)}{2}:=\tilde{k} .
$$


By Lemma 2.1.5,

$d_{k}^{\prime}:=f_{\lambda}\left(f_{\mu}\left(d_{k}\right)\right)>f_{\lambda}\left(f_{\mu}\left(\frac{1}{2}-\frac{1}{2 \sqrt[k]{\mu^{k-1} \lambda}}\right)\right), f_{\mu}(\underbrace{f_{\mu} \cdots f_{\mu}}_{k-2}\left(d_{k}^{\prime}\right))>d_{k}>\frac{1}{2}-\frac{1}{2 \sqrt[k]{\mu^{k-1} \lambda}}$.

After that, if case (III) 3 continues to happen till the process reaches $d_{k}^{\prime}$, then the largest derivative will be $M_{k}^{\prime}=\prod_{i=2}^{k} N_{k i}$. After that if the process keeps in the interval $\left(\frac{1}{2}-\frac{1}{2 \sqrt[k]{\mu^{k-1} \lambda}}, \frac{1}{2}-\frac{1}{2 \sqrt[k-1]{\mu^{k-2} \lambda}}\right]$, it will be case $(\mathrm{I})(4)(\mathrm{b})$. However, if case (III)(4) is repeated till the last point which leaves the interval $\left[1-\frac{1}{\mu}, \frac{1}{2}-\frac{1}{2 \sqrt[k]{\mu^{k-1} \lambda}}\right]$ is $f_{\mu}\left(d_{k}\right)$, see Figure 2.6. After that, for this same process, by Lemma 2.1.5, the number of $f_{\mu}^{\prime} s$ is at least $k$ times as many as the number of $f_{\lambda}^{\prime} s$. Again any $k f_{\mu}^{\prime} s$ and one $f_{\lambda}$ make the multiplication of their derivatives less than 1 , the largest of these is

$$
f_{\lambda}^{\prime}\left(1-\frac{1}{\mu}\right)\left(f_{\mu}^{\prime}\left(1-\frac{1}{\mu}\right)\right)^{k}<1
$$

So we have

$$
\frac{\partial\left(f_{\epsilon_{-1}} f_{\epsilon_{-2}} \cdots f_{\epsilon_{-n}}(\xi)\right)}{\partial \xi} \leqslant M_{k}^{\prime} L_{k+1}^{\left[\frac{n-\tilde{k}}{k+1}\right]}
$$

4. Consider $\omega$ such that the process enters $\left(\frac{1}{2}-\frac{1}{2 \sqrt[k-1]{\mu^{k-2} \lambda}}, \frac{1}{2}-\frac{1}{2 \sqrt[k-2]{\mu^{k-3} \lambda}}\right]$. Since $f_{\lambda}^{\prime}(x)\left(f_{\mu}^{\prime}(x)\right)^{k-2} \geqslant 1$ and $f_{\lambda}^{\prime}(x)\left(f_{\mu}^{\prime}(x)\right)^{k-1}<1$ for any $x \in\left(\frac{1}{2}-\frac{1}{2 \sqrt[k]{\mu^{k-1} \lambda}}, \frac{1}{2}-\right.$ $\left.\frac{1}{2 \sqrt[k-1]{\mu^{k-2} \lambda}}\right]$. So we can let $n_{k-1}$ denote the minimum number of $f_{\lambda}$ in the sequence

$$
f_{\lambda}(\underbrace{f_{\mu} \cdots f_{\mu}}_{k-2} f_{\lambda} \cdots \underbrace{f_{\mu} \cdots f_{\mu}}_{k-2} f_{\lambda}\left(\frac{1}{2}-\frac{1}{2 \sqrt[k]{\mu^{k-1} \lambda}}\right))>\frac{1}{2}-\frac{1}{2 \sqrt[k-1]{\mu^{k-2} \lambda}} .
$$

Then from time 0 to the first time it enters the interval $\left(\frac{1}{2}-\frac{1}{2 \sqrt[k-1]{\mu^{k-2} \lambda}}, \frac{1}{2}-\right.$ $\left.\frac{1}{2 \sqrt[k-1]{\mu^{k-2} \lambda}}\right]$, the number of $f_{\lambda}^{\prime} s$ is at most $n_{k-1}$ more than $\frac{1}{k-2}-t h$ of the number of $f_{\mu}^{\prime} s$. The derivative of this multiplication is $M_{k-1}^{n_{k-1}}$ and $M_{k-1}=f_{\lambda}^{\prime}\left(\frac{1}{2}-\frac{1}{2 \sqrt[k-1]{\mu^{k-2} \lambda}}\right)$.

5. If the process continue to jump along the $\mathrm{x}$-axis direction till it is into the interval $\left[\frac{1}{2}-\frac{1}{2 \lambda}, 1-\frac{1}{\lambda}\right]$ after it enters $\left(\frac{1}{2}-\frac{1}{2 \sqrt[k-1]{\mu^{k-2} \lambda}}, \frac{1}{2}-\frac{1}{2 \sqrt[k-2]{\mu^{k-3} \lambda}}\right]$, with the same reason as (III) 4 , we have

$$
\frac{\partial\left(f_{\epsilon_{-1}} f_{\epsilon_{-2}} \cdots f_{\epsilon_{-n}}(\xi)\right)}{\partial \xi} \leqslant M_{k-1}^{m_{k-1}} L_{k+1}^{\left[\frac{n-m_{k-1}}{k+1}\right]}
$$

where $m_{k-1}=n_{1}+n_{2} \cdots+n_{i}+\cdots+n_{k-1}$ and for $i=2, \cdots, k-1, n_{i}$ denote the minimum number of $f_{\lambda}$ in the following sequence

$$
f_{\lambda}(\underbrace{f_{\mu} \cdots f_{\mu}}_{i-1} f_{\lambda} \cdots \underbrace{f_{\mu} \cdots f_{\mu}}_{i-1} f_{\lambda}\left(\frac{1}{2}-\frac{1}{2 \sqrt[i+1]{\mu^{i} \lambda}}\right)>\frac{1}{2}-\frac{1}{2 \sqrt[i]{\mu^{i-1} \lambda}} .
$$


As a result, considering above five cases, for any $\xi \in\left(\frac{1}{2}-\frac{1}{2 \sqrt[k]{\mu^{k-1} \lambda}}, \frac{1}{2}-\frac{1}{2 \sqrt[k-1]{\mu^{k-2} \lambda}}\right]$, we have

$$
\frac{\partial\left(f_{\epsilon_{-1}} f_{\epsilon_{-2}} \cdots f_{\epsilon_{-n}}(\xi)\right)}{\partial \xi} \leqslant M_{k-1}^{m_{k-1}} \prod_{j=2}^{k} M_{j}^{\prime} L_{k+1}^{\left[\frac{n-n_{k-1}-\tilde{m}}{k+1}\right]}
$$

Here $M_{j}^{\prime}=\prod_{i=2}^{j} N_{j i}, N_{j i}=\frac{d}{d x}\left[f_{\lambda}(\underbrace{f_{\mu} \cdots f_{\mu}}_{i-1}\left(\frac{1}{2}-\frac{1}{2 \sqrt[j]{\mu^{j-1} \lambda}}\right))\right]$ and $\tilde{m}=\sum_{m=2}^{k}\left(\sum_{i=2}^{m} i\right)$.

Case (IV). $\xi \in\left[1-\frac{1}{\mu}, \frac{1}{2}-\frac{1}{2 \sqrt[k]{\mu^{k-1} \lambda}}\right]$

1. Consider $\omega$ such that the process starting at $\xi \in\left[1-\frac{1}{\mu}, \frac{1}{2}-\frac{1}{2 \sqrt[k]{\mu^{k-1} \lambda}}\right]$ stays in the interval forever. This case is same as the case (I)(4)(a).

2. consider the case the process starts at $\xi$. The process enters $\left(\frac{1}{2}-\frac{1}{2 \sqrt[k]{\mu^{k-1} \lambda}}, \frac{1}{2}-\right.$ $\left.\frac{1}{2 \sqrt[k-1]{\mu^{k-2} \lambda}}\right]$. Since $f_{\lambda}^{\prime}(x)\left(f_{\mu}^{\prime}(x)\right)^{k-1} \geqslant 1$ and $f_{\lambda}^{\prime}(x)\left(f_{\mu}^{\prime}(x)\right)^{k}<1$ for $\left[1-\frac{1}{\mu}, \frac{1}{2}-\right.$ $\left.\frac{1}{2 \sqrt[k]{\mu^{k-1} \lambda}}\right]$. So we can let $n_{k}$ denote the minimum number of $f_{\lambda}$ in the sequence

$$
f_{\lambda}(\underbrace{f_{\mu} \cdots f_{\mu}}_{k-1} f_{\lambda} \cdots \underbrace{f_{\mu} \cdots f_{\mu}}_{k-1} f_{\lambda}\left(1-\frac{1}{\mu}\right))>\frac{1}{2}-\frac{1}{2 \sqrt[k]{\mu^{k-1} \lambda}} .
$$

Then from time 0 to the first time it enters the interval $\left(\frac{1}{2}-\frac{1}{2 \sqrt[k]{\mu^{k-1} \lambda}}, \frac{1}{2}-\frac{1}{2 \sqrt[k-1]{\mu^{k-2} \lambda}}\right]$, the number of $f_{\lambda}^{\prime} s$ is at most $n_{k}$ more than $\frac{1}{k-1}-t h$ of the number of $f_{\mu}^{\prime} s$. After that, it will repeat case $(\mathrm{I})(4)(\mathrm{b})$ and $(\mathrm{I})(4)(\mathrm{c})$. So we have

$$
\frac{\partial\left(f_{\epsilon_{-1}} f_{\epsilon_{-2}} \cdots f_{\epsilon_{-n}}(\xi)\right)}{\partial \xi} \leqslant M_{k}^{n_{k}} L_{k+1}^{\left[\frac{n-n_{k}}{k+1}\right]}
$$

where $M_{k}=f_{\lambda}^{\prime}\left(1-\frac{1}{\mu}\right)$.

3. If the process $\xi \in\left[1-\frac{1}{\mu}, \frac{1}{2}-\frac{1}{2 \sqrt[k]{\mu^{k-1} \lambda}}\right]$ can be mapped into $\left(\frac{1}{2}-\frac{1}{2 \sqrt[k]{\mu^{k-1} \lambda}}, \frac{1}{2}-\right.$ $\left.\frac{1}{2 \sqrt[k-1]{\mu^{k-2} \lambda}}\right]$ and then continue to move towards the right direction till it reaches the internal $\left[\frac{1}{2}-\frac{1}{2 \lambda}, 1-\frac{1}{\lambda}\right]$ as Figure 2.5 shows, with the same reason as case (III) 3 , by Lemma 2.1.5, we have

$$
\frac{\partial\left(f_{\epsilon_{-1}} f_{\epsilon_{-2}} \cdots f_{\epsilon_{-n}}(\xi)\right)}{\partial \xi} \leqslant M_{k}^{m_{k}} \prod_{j=2}^{k} M_{j}^{\prime} L_{k+1}^{\left[\frac{n-m_{k}-\tilde{m}}{k+1}\right]},
$$

where $m_{k}=n_{1}+n_{2} \cdots+n_{i}+\cdots+n_{k}$ and for $i=2, \cdots, k-1$.

Concluding above three cases, we can conclude that for any $\xi \in\left[1-\frac{1}{\mu}, \frac{1}{2}-\frac{1}{2 \sqrt[k]{\mu^{k-1} \lambda}}\right]$, inequality (2.22) always holds. 
In conclusion Case (I) to Case (IV), when $1<\mu<\lambda \leqslant 2$, for any $x, y \in\left[1-\frac{1}{\mu}, 1-\frac{1}{\lambda}\right]$, we can always have the following inequality:

$$
\begin{aligned}
\left|f_{\epsilon_{-1}} \cdots f_{\epsilon_{-n}}(x)-f_{\epsilon_{-1}} \cdots f_{\epsilon_{-n}}(y)\right| & \leqslant \frac{\partial\left(f_{\epsilon_{-1}} \cdots f_{\epsilon_{-n}}(\xi)\right)}{\partial \xi}|x-y| \\
& \leqslant M_{k}^{m_{k}} \prod_{j=2}^{k} M_{j}^{\prime} L_{k+1}^{\left[\frac{n-m_{k}-\tilde{m}}{k+1}\right]}|x-y| .
\end{aligned}
$$

Limit of pullback when $1<\mu<\lambda \leqslant 2$.

Consequently, when $1<\mu<\lambda \leqslant 2$, if we choose $x=1-\frac{1}{\lambda}$ and $y=f_{\epsilon_{-(n+1)}} \cdots f_{\epsilon_{-(n+m)}}(1-$ $\left.\frac{1}{\lambda}\right)$, in $(2.23)$, we can get

$$
\begin{aligned}
& \left|f_{\epsilon_{-1}} f_{\epsilon_{-2}} \cdots f_{\epsilon_{-n}}\left(1-\frac{1}{\lambda}\right)-f_{\epsilon_{-1}} f_{\epsilon_{-2}} \cdots f_{\epsilon_{-n}}\left(f_{\epsilon_{-(n+1)}} \cdots f_{\epsilon_{-(n+m)}}\left(1-\frac{1}{\lambda}\right)\right)\right| \\
& \leqslant M_{k}^{m} \prod_{j=2}^{k} M_{j}^{\prime} L_{k+1}^{\left[\frac{n-m_{k}-\tilde{m}}{k+1}\right]}\left|1-\frac{1}{\lambda}-f_{\epsilon_{-(n+1)}} \cdots f_{\epsilon_{-(n+m)}}\left(1-\frac{1}{\lambda}\right)\right| \rightarrow 0,
\end{aligned}
$$

as $n \rightarrow \infty$.

In conclusion, $f_{\epsilon_{-1}} f_{\epsilon_{-2}} \cdots f_{\epsilon_{-n}}\left(1-\frac{1}{\lambda}\right)$ is a Cauchy sequence in $\left[1-\frac{1}{\mu}, 1-\frac{1}{\lambda}\right]$. So there is a limit, denoted by $Y^{\star}(\omega)$. Also for

$$
\begin{aligned}
\left|f_{\epsilon_{-1}} f_{\epsilon_{-2}} \cdots f_{\epsilon_{-n}}(x)-Y^{\star}(\omega)\right| \leqslant & \left|f_{\epsilon_{-1}} f_{\epsilon_{-2}} \cdots f_{\epsilon_{-n}}(x)-f_{\epsilon_{-1}} f_{\epsilon_{-2}} \cdots f_{\epsilon_{-n}}\left(1-\frac{1}{\lambda}\right)\right| \\
& +\left|f_{\epsilon_{-1}} f_{\epsilon_{-2}} \cdots f_{\epsilon_{-n}}\left(1-\frac{1}{\lambda}\right)-Y^{\star}(\omega)\right| \rightarrow 0 .
\end{aligned}
$$

Therefore, we can get

$$
\lim _{n \rightarrow \infty} f_{\epsilon_{-1}} f_{\epsilon_{-2}} \cdots f_{\epsilon_{-n}}(x)=Y^{\star}(\omega),
$$

which is independent of $x$. And Proposition 2.1.6 is proved.

We now consider the case $2<\mu<\lambda \leqslant 3$. We need the following lemma and we will leave the proof to section 4 .

Lemma 2.1.7 If $\mu \in(2, \lambda)$ and $\lambda \in[1+\sqrt{3}, 3]$, then for any $\epsilon_{1}$ and $\epsilon_{2}$, we have

$$
\left|f_{\epsilon_{2}}^{\prime}\left(f_{\epsilon_{1}}(x)\right) f_{\epsilon_{1}}^{\prime}(x)\right| \leqslant 1, \quad \forall x \in\left[\frac{\mu \lambda(4-\lambda)}{16}, \frac{\lambda}{4}\right] .
$$

Only when $\lambda=2$ and $x=\frac{2}{3}$, equality in (2.24) holds.

Proposition 2.1.8 Assume $2<\mu<\lambda \leqslant 3$. Then for any $x \in\left[1-\frac{1}{\mu}, 1-\frac{1}{\lambda}\right], \lim _{k \rightarrow \infty} f_{\epsilon_{-1}} \cdots f_{\epsilon_{-k}}(x)$ exists and the limit is independent of $x$.

Proof. It is easy to see that $\left[\frac{1}{2}, \frac{\lambda}{4}\right]$ is an interval on which $f_{\mu}$ and $f_{\lambda}$ are monotone and this interval is left invariant by $f_{\mu}$ and $f_{\lambda}$, i.e. $f_{\mu}\left[\frac{1}{2}, \frac{\lambda}{4}\right] \subset\left[\frac{1}{2}, \frac{\lambda}{4}\right]$ and $f_{\lambda}\left[\frac{1}{2}, \frac{\lambda}{4}\right] \subset\left[\frac{1}{2}, \frac{\lambda}{4}\right]$.

More precisely, for any $2<\mu<\lambda \leqslant 3$, the invariant interval will be $\left[\frac{\mu \lambda(4-\lambda)}{16}, \frac{\lambda}{4}\right]$. This means that once the process enters the interval $\left[\frac{\mu \lambda(4-\lambda)}{16}, \frac{\lambda}{4}\right]$, it will remain in it 
forever under the action of $f_{\mu}$ and $f_{\lambda}$. At the same time, since $2<\mu<\lambda \leqslant 3$, we have $\frac{\mu \lambda(4-\lambda)}{16}>\frac{1}{2}-\frac{1}{2 \lambda}$ since

$$
\frac{\mu \lambda(4-\lambda)}{16}-\left(\frac{1}{2}-\frac{1}{2 \lambda}\right)>\frac{\lambda(4-\lambda)}{8}-\frac{\lambda-1}{2 \lambda}=\frac{4 \lambda^{2}-\lambda^{3}-4 \lambda+4}{8 \lambda}>0 .
$$

We consider the following three cases.

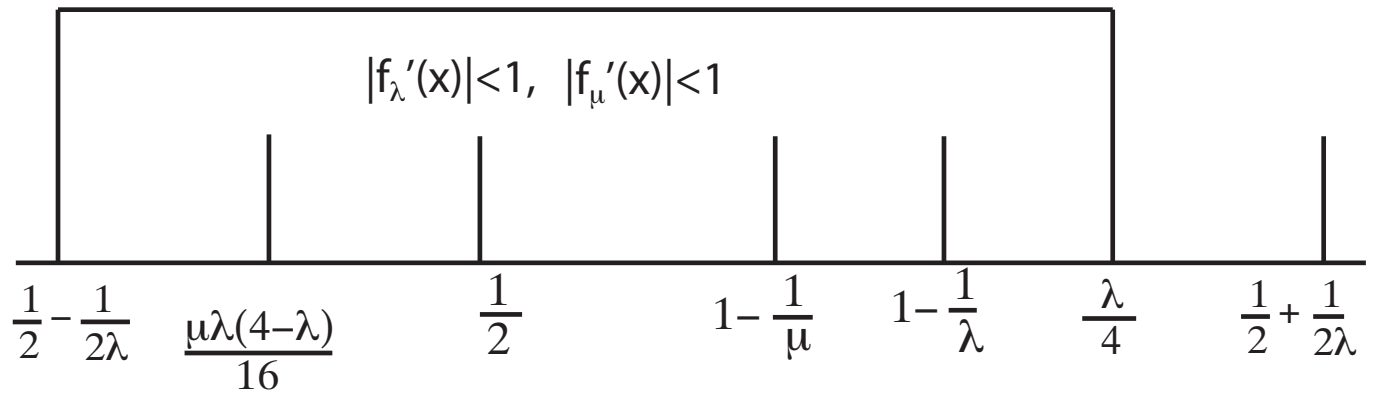

Figure 2.7: $2<\lambda \leqslant 1+\sqrt{3}$

1. When $2<\mu<\lambda<1+\sqrt{3}$ as Figure 2.7 shows, we have $\frac{\lambda}{4}<\frac{1}{2}+\frac{1}{2 \lambda}$. Note $\left|f_{\mu}^{\prime}(x)\right|<1$ and $\left|f_{\lambda}^{\prime}(x)\right|<1$ for any $x \in\left[\frac{\mu \lambda(4-\lambda)}{16}, \frac{\lambda}{4}\right] \subset\left[\frac{\mu \lambda(4-\lambda)}{16}, \frac{1}{2}+\frac{1}{2 \lambda}\right]$. So for any $x, y \in\left[\frac{\mu \lambda(4-\lambda)}{16}, \frac{\lambda}{4}\right]$, it is easy to get

$$
\left|f_{\epsilon_{-1}} f_{\epsilon_{-2}} \cdots f_{\epsilon_{-(n-1)}} f_{\epsilon_{-n}}(x)-f_{\epsilon_{-1}} f_{\epsilon_{-2}} \cdots f_{\epsilon_{-(n-1)}} f_{\epsilon_{-n}}(y)\right| \leqslant L^{n}|x-y|,
$$

where $L=\max \left\{\left|f_{\lambda}^{\prime}\left(\frac{\lambda}{4}\right)\right|,\left|f_{\lambda}^{\prime}\left(\frac{\mu \lambda(4-\lambda)}{16}\right)\right|\right\}<1$.

2. When $2<\mu<\lambda$ and $1+\sqrt{3} \leqslant \lambda<3$, from the proof of Lemma 2.1.7, as Figure 2.8 shows, we can get

$$
\left|f_{\epsilon_{-1}} f_{\epsilon_{-2}} \cdots f_{\epsilon_{-(n-1)}} f_{\epsilon_{-n}}(x)-f_{\epsilon_{-1}} f_{\epsilon_{-2}} \cdots f_{\epsilon_{-(n-1)}} f_{\epsilon_{-n}}(y)\right| \leqslant \bar{L}^{\left[\frac{n}{2}\right]}|x-y|,
$$

where

$$
\begin{aligned}
\bar{L}= & \max \left\{\left|f_{\lambda}^{\prime}\left(f_{\lambda}\left(\frac{1}{2}+\frac{\sqrt{3 \lambda^{2}-6 \lambda}}{6 \lambda}\right)\right) f_{\lambda}^{\prime}\left(\frac{1}{2}+\frac{\sqrt{3 \lambda^{2}-6 \lambda}}{6 \lambda}\right)\right|,\left|f_{\lambda}^{\prime}\left(f_{\mu}\left(\frac{\mu \lambda(4-\lambda)}{16}\right)\right) f_{\mu}^{\prime}\left(\frac{\mu \lambda(4-\lambda)}{16}\right)\right|,\right. \\
& \left.\left|f_{\lambda}^{\prime}\left(f_{\mu}\left(\frac{1}{2} \pm \frac{\sqrt{3 \mu^{2}-6 \mu}}{6 \mu}\right)\right) f_{\mu}^{\prime}\left(\frac{1}{2} \pm \frac{\sqrt{3 \mu^{2}-6 \mu}}{6 \mu}\right)\right|,\left|f_{\lambda}^{\prime}\left(f_{\mu}\left(\frac{\lambda}{4}\right)\right) f_{\mu}^{\prime}\left(\frac{\lambda}{4}\right)\right|\right\}<1 .
\end{aligned}
$$

3. When $2<\mu<\lambda=3, \frac{1}{2}+\frac{\sqrt{3 \lambda^{2}-6 \lambda}}{6 \lambda}=\frac{2}{3}$ and $\frac{\mu \lambda(4-\lambda)}{16}=\frac{3 \mu}{16}$. We have $\left|f_{\lambda}^{\prime}\left(\frac{2}{3}\right)\right|=1$ and $\left|f_{\lambda}^{\prime}\left(f_{\lambda}\left(\frac{2}{3}\right)\right) f_{\lambda}^{\prime}\left(\frac{2}{3}\right)\right|=1$, and $\left|f_{\epsilon_{1}}^{\prime}\left(f_{\epsilon_{2}}(x)\right) f_{\epsilon_{2}}^{\prime}(x)\right|<1$ for any $x \in\left[\frac{3 \mu}{16}, \frac{2}{3}\right) \cup\left(\frac{2}{3}, \frac{\lambda}{4}\right]$. In the sequence $f_{\epsilon_{-1}} f_{\epsilon_{-2}} \cdots f_{\epsilon_{-(n-1)}} f_{\epsilon_{-n}}(x)$, the number of terms $f_{\lambda}\left(f_{\lambda}(x)\right)$ is denoted by $k_{1}$. Then obviously, $k_{1}<\frac{n}{2}$. Moreover, for the other $\left[\frac{n}{2}\right]-k_{1}$ terms, in every pair $f_{\epsilon_{1}}\left(f_{\epsilon_{2}}(x)\right)$, we have $\epsilon_{1}=\mu$ or $\epsilon_{2}=\mu$. Since there are infinite $f_{\mu}^{\prime} s$ in the sequence $f_{\epsilon_{-1}} f_{\epsilon_{-2}} \cdots f_{\epsilon_{-(n-1)}} f_{\epsilon_{-n}}(x)$ as $n \rightarrow \infty$, so $\left[\frac{n}{2}-k_{1}\right]$ must go to infinity. Then it is obvious that

$$
\left|f_{\epsilon_{-1}} f_{\epsilon_{-2}} \cdots f_{\epsilon_{-(n-1)}} f_{\epsilon_{-n}}(x)-f_{\epsilon_{-1}} f_{\epsilon_{-2}} \cdots f_{\epsilon_{-(n-1)}} f_{\epsilon_{-n}}(y)\right| \leqslant L_{1}^{k_{1}} L_{2}^{\left[\frac{n}{2}\right]-k_{1}}|x-y|,
$$




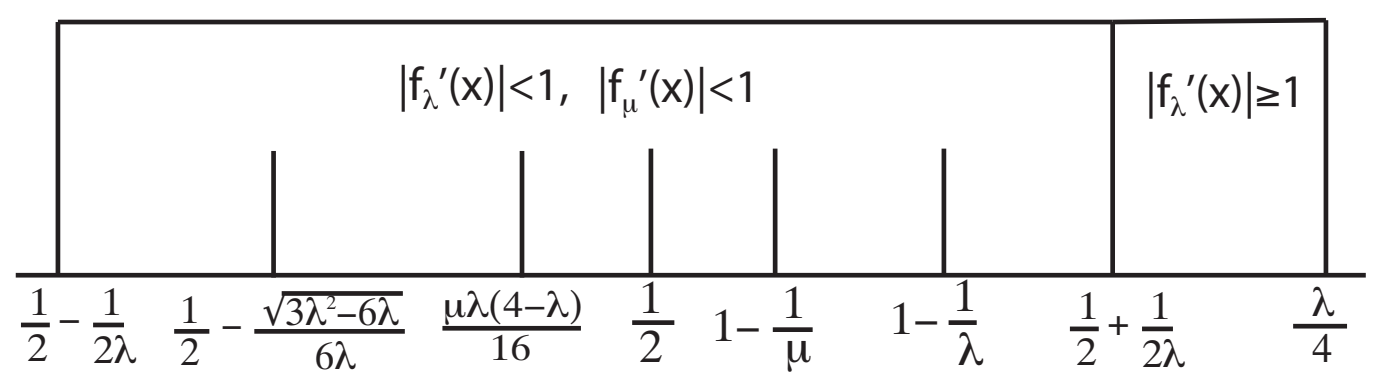

Figure 2.8: $1+\sqrt{3}<\lambda \leqslant 3$

where $L_{1}=\max \left\{\left|f_{\lambda}^{\prime}\left(f_{\lambda}(x)\right) f_{\lambda}^{\prime}(x)\right|\right\} \leqslant 1$. Because it is easy to see that

$$
\left|f_{\mu}^{\prime}\left(f_{\mu}(x)\right) f_{\mu}^{\prime}(x)\right|<\left|f_{\lambda}^{\prime}\left(f_{\mu}(x)\right) f_{\mu}^{\prime}(x)\right|,
$$

so according to (4.16), we can take

$$
\begin{aligned}
L_{2}= & \max \left\{\left|f_{\lambda}^{\prime}\left(f_{\mu}\left(\frac{1}{2} \pm \frac{\sqrt{3 \mu^{2}-6 \mu}}{6 \mu}\right)\right) f_{\mu}^{\prime}\left(\frac{1}{2} \pm \frac{\sqrt{3 \mu^{2}-6 \mu}}{6 \mu}\right)\right|,\left|f_{\lambda}^{\prime}\left(f_{\mu}\left(\frac{\mu \lambda(4-\lambda)}{16}\right)\right) f_{\mu}^{\prime}\left(\frac{\mu \lambda(4-\lambda)}{16}\right)\right|,\right. \\
& \left.\left|f_{\lambda}^{\prime}\left(f_{\mu}\left(\frac{\lambda}{4}\right)\right) f_{\mu}^{\prime}\left(\frac{\lambda}{4}\right)\right|,\left|f_{\mu}^{\prime}\left(f_{\lambda}\left(\frac{1}{2}+\frac{\sqrt{3 \lambda^{2}-6 \lambda}}{6 \lambda}\right)\right) f_{\lambda}^{\prime}\left(\frac{1}{2}+\frac{\sqrt{3 \lambda^{2}-6 \lambda}}{6 \lambda}\right)\right|\right\}<1 .
\end{aligned}
$$

In conclusion, with the same reason as Proposition 2.1.6, we can conclude that the limit of $f_{\epsilon_{-1}} f_{\epsilon_{-2}} \cdots f_{\epsilon_{-k}}(x)$ exists as $k \rightarrow \infty$ for $x \in\left[1-\frac{1}{\mu}, 1-\frac{1}{\lambda}\right] \in\left[\frac{\mu \lambda(4-\lambda)}{16}, \frac{\lambda}{4}\right]$.

Now we consider the case $1<\mu \leqslant 2<\lambda \leqslant 3$, which can be divided into two cases: $\frac{4}{\lambda}<\mu \leqslant 2$ and $1<\mu \leqslant \frac{4}{\lambda}$. For the former case, we need Lemma 2.1.9.

Lemma 2.1.9 For $\frac{4}{\lambda}<\mu \leqslant 2,2<\lambda \leqslant 3$, we have

$$
\left|f_{\epsilon_{2}}^{\prime}\left(f_{\epsilon_{1}}(x)\right) f_{\epsilon_{1}}^{\prime}(x)\right| \leqslant 1, \quad \forall x \in\left[\frac{\mu \lambda(4-\lambda)}{16}, \frac{\lambda}{4}\right] .
$$

As for the later case, it is easy to see that for any $1<\mu \leqslant \frac{4}{\lambda}, 2<\lambda \leqslant 3$, there exists an integer $k>0$ such that $\frac{\mu}{(2-\mu)^{k}} \leqslant \lambda<\frac{\mu}{(2-\mu)^{k+1}}$. We need the following lemmas.

Lemma 2.1.10 If $1<\mu \leqslant \frac{4}{\lambda}$ and $2<\lambda \leqslant 3$, then for any $x \in\left[1-\frac{1}{\mu}, \frac{1}{2}-\frac{1}{2 \lambda}\right]$,

$$
f_{\lambda}\left(f_{\mu}(x)\right)>x .
$$

See Figure 2.9.

Lemma 2.1.11 If $1<\mu<\frac{4}{\lambda}$ and $2<\lambda<\frac{7}{3}$, then for any $x \in\left[1-\frac{1}{\mu}, a_{2}\right], f_{\lambda}\left(f_{\mu}\left(f_{\mu}(x)\right)\right)>$ $x$, where $a_{2}$ is defined by $f_{\mu}\left(f_{\mu}\left(a_{2}\right)\right)=\frac{1}{2}-\frac{1}{2 \sqrt{\mu \lambda}}$.

If $1<\mu<\frac{4}{\lambda}$ and $\frac{7}{3} \leqslant \lambda \leqslant 3$,

$$
f_{\lambda}\left(f_{\mu}\left(\frac{1}{2}-\frac{1}{2 \sqrt{\mu \lambda}}\right)\right)>\frac{1}{2}-\frac{1}{2 \lambda} .
$$

See Figure 2.9. 


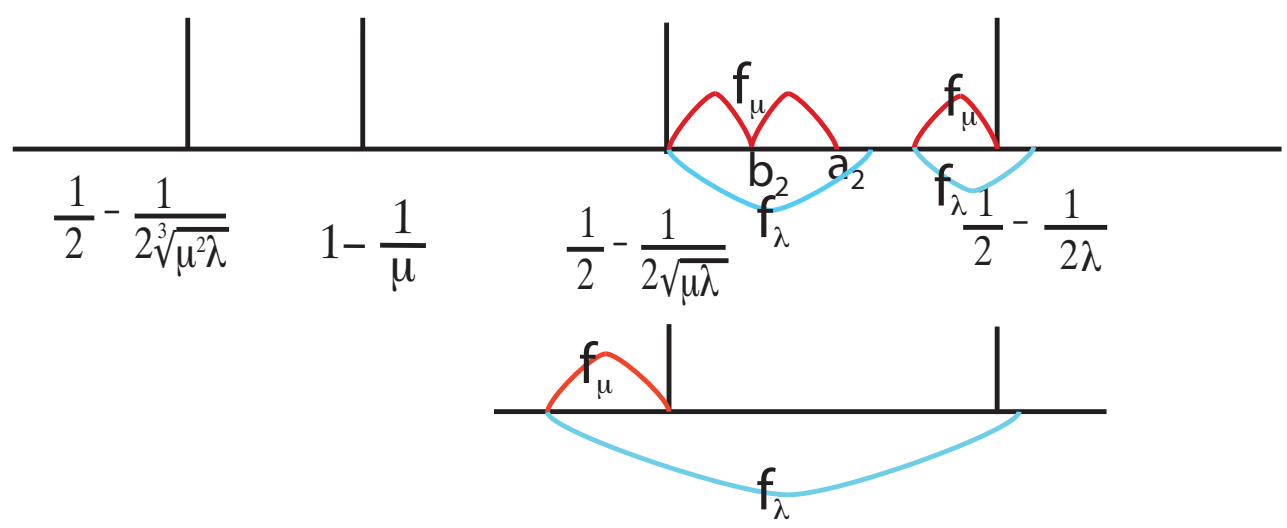

Figure 2.9: $\lambda \geqslant \frac{\mu}{(2-\mu)^{2}}$

Lemma 2.1.12 Assume $\lambda \geqslant \frac{\mu}{(2-\mu)^{2}}$, and $b_{2}$ is defined by $f_{\mu}\left(b_{2}\right)=\frac{1}{2}-\frac{1}{2 \sqrt{\mu \lambda}}$. Then for $x \in\left[1-\frac{1}{\mu}, b_{2}\right]$, the following inequality holds:

$$
f_{\lambda}\left(f_{\mu}\left(f_{\mu}(x)\right)\right)>x
$$

See Figure 2.10.

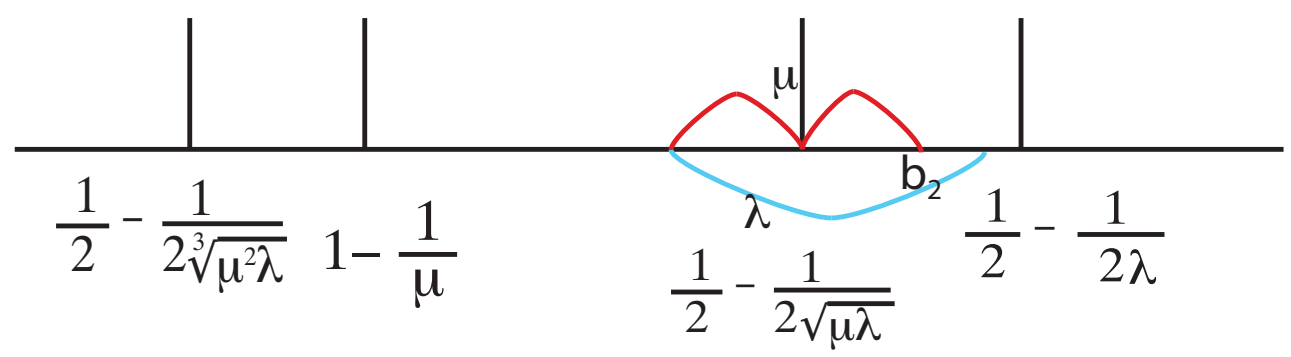

Figure 2.10: $f_{\lambda}\left(f_{\mu}\left(f_{\mu}\left(b_{2}\right)\right)\right)>b_{2}$

Lemma 2.1.13 Assume $1<\mu \leqslant \frac{4}{\lambda}, 2<\lambda \leqslant 3$, and $\lambda \geqslant \frac{\mu}{(2-\mu)^{k}}$. Then for any $x \in$ $\left[1-\frac{1}{\mu}, b_{k}\right]$ and $k \geqslant 2$,

$$
f_{\lambda}(\underbrace{f_{\mu} \cdots f_{\mu}}_{k}(x))>x
$$

where $\underbrace{f_{\mu} \cdots f_{\mu}}_{k-1}\left(b_{k}\right)=\frac{1}{2}-\frac{1}{2 \sqrt[k]{\mu^{k-1} \lambda}}$. See Figure 2.11.

Lemma 2.1.14 When $k \geqslant 3, \frac{d}{d x}[f_{\lambda} \underbrace{f_{\mu} \cdots f_{\mu}}_{k-1}(x)]<1$ for $x \in\left[c_{k}, b_{k}\right]$, where $b_{k}$ is defined in the same way as in Lemma 2.1.13 and $c_{k}=f_{\mu}\left(b_{k}\right)$.

See Figure 2.11. 


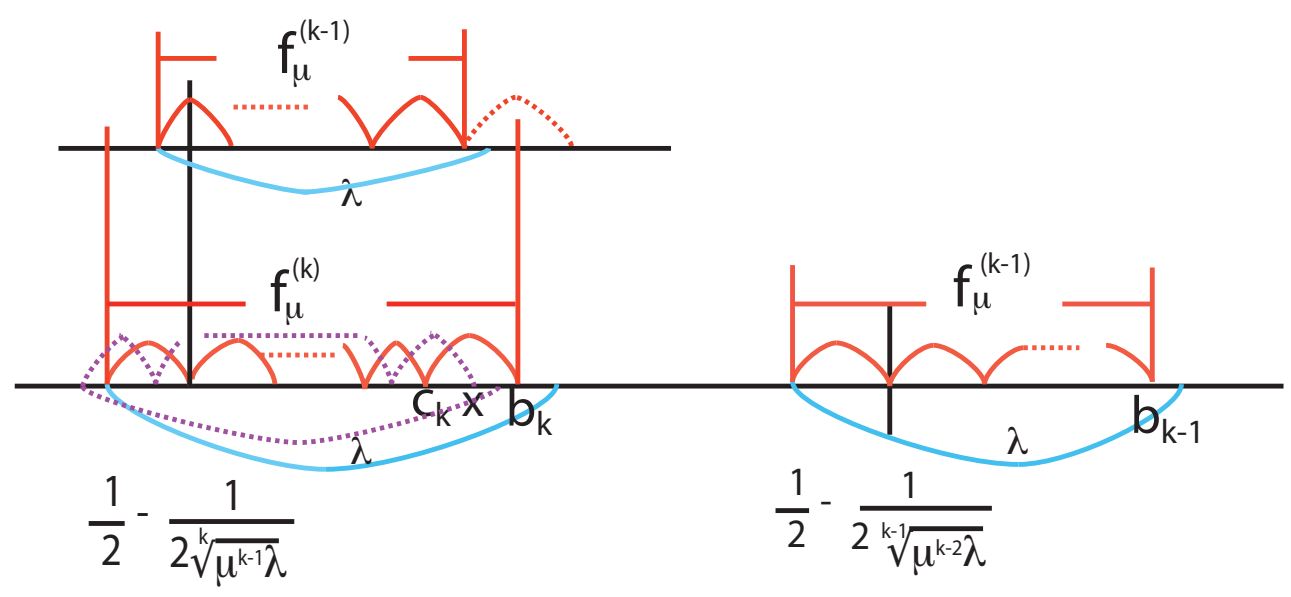

Figure 2.11: $\lambda \geqslant \frac{\mu}{(2-\mu)^{k}}$ when $1<\mu \leqslant \frac{4}{\lambda}, 2<\lambda \leqslant 3$

Lemma 2.1.15 When $1<\mu \leqslant 2<\lambda \leqslant 3$, for any $k \geqslant 2$, we have the following inequality

$$
f_{\lambda}\left(\frac{1}{2}-\frac{1}{2 \sqrt[k]{\mu^{k-1} \lambda}}\right)>\frac{1}{2}-\frac{1}{2 \sqrt[k-1]{\mu^{k-2} \lambda}} .
$$

Proposition 2.1.16 Assume $1<\mu \leqslant 2<\lambda \leqslant 3$. Then for any $x \in\left[1-\frac{1}{\mu}, 1-\frac{1}{\lambda}\right]$, $\lim _{k \rightarrow \infty} f_{\epsilon_{-1}} f_{\epsilon_{-2}} \cdots f_{\epsilon_{-k}}(x)$ exists and the limit is independent of $x$.

Proof.

(1). When $\mu>\frac{4}{\lambda}$, it is obvious that $1-\frac{1}{\mu}>\frac{\mu \lambda(4-\lambda)}{16}$. This case is similar to the case $2<\mu<\lambda \leqslant 3$. According to (4.18), (4.19) and (4.20) in the proof of Lemma 2.1.9, which can be seen in section 4.4.1, when $\mu \in\left(\frac{4}{\lambda}, \lambda\right)$ and $\lambda \in(2,3)$, for any $x, y \in\left[\frac{\mu \lambda(4-\lambda)}{16}, \frac{\lambda}{4}\right]$, we can get

$$
\left|f_{\epsilon_{-1}} f_{\epsilon_{-2}} \cdots f_{\epsilon_{-(n-1)}} f_{\epsilon_{-n}}(x)-f_{\epsilon_{-1}} f_{\epsilon_{-2}} \cdots f_{\epsilon_{-(n-1)}} f_{\epsilon_{-n}}(y)\right| \leqslant \tilde{L}_{1}^{\left[\frac{n}{2}\right]}|x-y|,
$$

where $\tilde{L_{1}}=\max \left\{L_{1}^{\prime}, L_{2}^{\prime}\right\}<1$ and $L_{1}^{\prime}, L_{2}^{\prime}$ are defined in section 4.4.1.

While when $\lambda=3$, with the same reason as the case $2<\mu<\lambda=3$, we can get

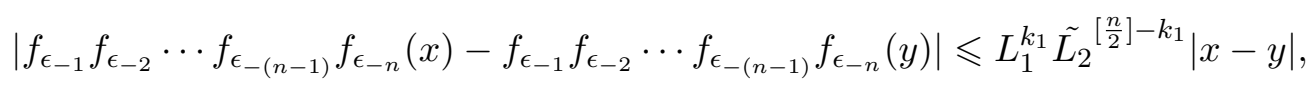

where $L_{1}$ is the same as in (2.27) and

$$
\tilde{L_{2}}=\max \left\{L_{2}^{\prime}, L_{3}^{\prime}\right\}<1 .
$$

The contents $L_{2}^{\prime}, L_{3}^{\prime}$ will be given in 4.4.1.

(2). On the other hand, when $\mu \leqslant \frac{4}{\lambda}$, the proof is similar to the case $1<\mu<\lambda \leqslant 2$. We need the following lemmas. Proof is same as the case $1<\mu<\lambda \leqslant 2$, we will first discuss $\xi \in\left[\frac{1}{2}-\frac{1}{2 \lambda}, 1-\frac{1}{\lambda}\right]$. 


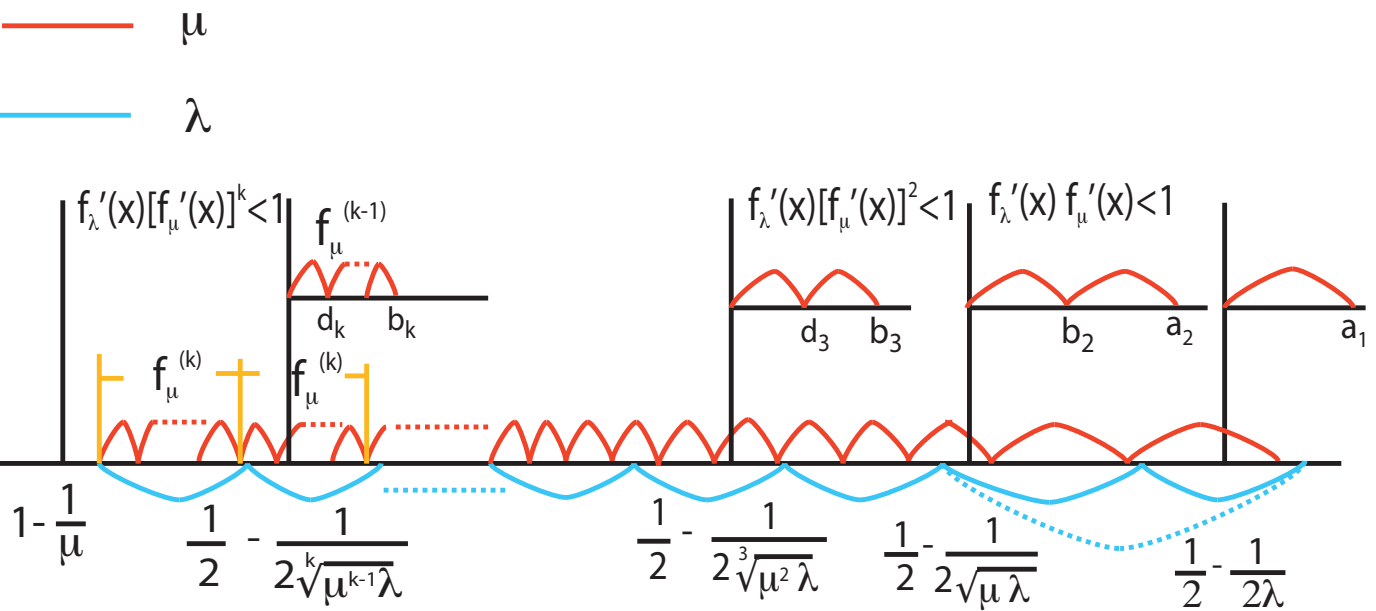

Figure 2.12: Contracted process

(I). $\xi \in\left[\frac{1}{2}-\frac{1}{2 \lambda}, 1-\frac{1}{\lambda}\right]$.

(i) For any $\xi \in\left[\frac{1}{2}-\frac{1}{2 \lambda}, 1-\frac{1}{\lambda}\right]$, if the process stays in the interval, then we have $\left|f_{\epsilon_{k}}^{\prime}(x)\right| \leqslant 1$. The multiplication of the derivatives of any pair of function is less than or equal to

$$
L_{1}=\max \left\{\left|f_{\mu}^{\prime}\left(a_{1}\right)\right|,\left|f_{\lambda}^{\prime}\left(f_{\lambda}\left(\frac{1}{2}-\frac{1}{2 \lambda}\right)\right)\right|\right\}<1,
$$

where $a_{1}$ is defined by $f_{\mu}\left(a_{1}\right)=\frac{1}{2}-\frac{1}{2 \lambda}$. Then for any $\xi \in\left[\frac{1}{2}-\frac{1}{2 \lambda}, 1-\frac{1}{\lambda}\right]$, we can get

$$
\left|\frac{d}{d \xi}\left[f_{\epsilon_{-1}} f_{\epsilon_{-2}} \cdots f_{\epsilon_{-n}}(\xi)\right]\right| \leqslant L_{1}^{\left[\frac{n}{2}\right]} .
$$

(ii) For $\xi \in\left[\frac{1}{2}-\frac{1}{2 \lambda}, 1-\frac{1}{\lambda}\right]$, if the process is mapped by $f_{\mu}$ to $\left(\frac{1}{2}-\frac{1}{2 \sqrt{\mu \lambda}}, \frac{1}{2}-\frac{1}{2 \lambda}\right)$ and then comes back to $\left[\frac{1}{2}-\frac{1}{2 \lambda}, 1-\frac{1}{\lambda}\right]$ without going into $\left[1-\frac{1}{\mu}, \frac{1}{2}-\frac{1}{2 \sqrt{\mu \lambda}}\right]$. By Lemma 2.1.2 and Lemma 2.1.3, in the sequence from the time it leaves the interval $\left[\frac{1}{2}-\frac{1}{2 \lambda}, 1-\frac{1}{\lambda}\right]$ to the first time it comes back to the interval, it is obvious that the number of $f_{\mu}^{\prime} s$ is more than or equal to the number of $f_{\lambda}^{\prime} s$. But note also any pair of $f_{\mu}$ and $f_{\lambda}$ makes the multiplication of their derivative less than 1 . The largest of these multiplication is

$$
L_{2}^{\prime}=f_{\lambda}^{\prime}\left(\frac{1}{2}-\frac{1}{2 \sqrt{\mu \lambda}}\right) f_{\mu}^{\prime}\left(b_{2}\right)<1 .
$$

After that, it will be the case (1) again.

However, if $\xi \in\left[\frac{1}{2}-\frac{1}{2 \lambda}, 1-\frac{1}{\lambda}\right]$ is mapped by $f_{\mu}$ to $\left(\frac{1}{2}-\frac{1}{2 \sqrt{\mu \lambda}}, \frac{1}{2}-\frac{1}{2 \lambda}\right)$ and stays in the interval forever. Then the number of $f_{\mu}^{\prime} s$ is more than the number of $f_{\lambda}^{\prime} s$ in the sequence. As before, any pair of $f_{\mu}$ and $f_{\lambda}$ makes the multiplication of their derivatives less than 1 . The largest of them is again $L_{2}^{\prime}$. 
Therefore, in this case, we have for any $\xi \in\left[\frac{1}{2}-\frac{1}{2 \lambda}, 1-\frac{1}{\lambda}\right]$,

$$
\left|\frac{d}{d \xi}\left[f_{\epsilon_{-1}} f_{\epsilon_{-2}} \cdots f_{\epsilon_{-n}}(\xi)\right]\right| \leqslant L_{2}^{\left[\frac{n}{2}\right]}
$$

where $L_{2}=\max \left\{L_{2}^{\prime}, L_{1}\right\}<1$.

(iii) Starting from $\xi \in\left[\frac{1}{2}-\frac{1}{2 \lambda}, 1-\frac{1}{\lambda}\right]$, if the process is mapped by $f_{\mu}$ to $\left(\frac{1}{2}-\frac{1}{2 \sqrt{\mu \lambda}}, \frac{1}{2}-\frac{1}{2 \lambda}\right)$ and then goes to the interval $\left(\frac{1}{2}-\frac{1}{2 \sqrt[3]{\mu^{2} \lambda}}, \frac{1}{2}-\frac{1}{2 \sqrt{\mu \lambda}}\right]$. There are two subcases.

(a) If the above step is followed immediately by the map $f_{\lambda}$, we consider cases $2<\lambda<\frac{7}{3}$ and $\frac{7}{3} \leqslant \lambda \leqslant 3$ respectively.

By Lemma 2.1.10 and Lemma 2.1.11, the case $2<\lambda<\frac{7}{3}$ is the same as the case (I)(3)(b) when $1<\mu<\lambda \leqslant 2$. The largest derivative is $f_{\lambda}^{\prime}\left(\frac{1}{2}-\right.$ $\left.\frac{1}{2 \sqrt[3]{\mu^{2} \lambda}}\right)\left(f_{\mu}^{\prime}\left(d_{3}\right)\right)^{2}<1$, where $f_{\mu}\left(d_{3}\right)=\frac{1}{2}-\frac{1}{2 \sqrt[3]{\mu^{2} \lambda}}$.

In the second case $\frac{7}{3} \leqslant \lambda \leqslant 3$, from (2.30), as Figure 2.9 shows, we know the map $f_{\lambda}$ will directly take a point $x$ to the interval $\left[\frac{1}{2}-\frac{1}{2 \lambda}, 1-\frac{1}{\lambda}\right]$ when $x \geqslant f_{\mu}\left(\frac{1}{2}-\frac{1}{2 \sqrt{\mu \lambda}}\right)$ as Figure 2.9 shows. From the first time that the process starting at $\xi \in\left[\frac{1}{2}-\frac{1}{2 \lambda}, 1-\frac{1}{\lambda}\right]$ enters $\left[f_{\mu}\left(\frac{1}{2}-\frac{1}{2 \sqrt{\mu \lambda}}\right), \frac{1}{2}-\frac{1}{2 \sqrt{\mu \lambda}}\right]$ to the time that the process comes back to $\left[\frac{1}{2}-\frac{1}{2 \lambda}, 1-\frac{1}{\lambda}\right]$, the largest derivative is

$$
\max \left\{f_{\lambda}^{\prime}\left(f_{\mu}\left(\frac{1}{2}-\frac{1}{2 \lambda}\right)\right) f_{\mu}^{\prime}\left(\frac{1}{2}-\frac{1}{2 \lambda}\right), f_{\lambda}^{\prime}\left(f_{\mu}\left(\frac{1}{2}-\frac{1}{2 \sqrt{\mu \lambda}}\right)\right) f_{\mu}^{\prime}\left(\frac{1}{2}-\frac{1}{2 \sqrt{\mu \lambda}}\right) f_{\mu}^{\prime}\left(b_{2}\right)\right\} .
$$

According to Lemma 2.1.2, we have

$f_{\lambda}^{\prime}\left(f_{\mu}\left(\frac{1}{2}-\frac{1}{2 \sqrt{\mu \lambda}}\right)\right) f_{\mu}^{\prime}\left(\frac{1}{2}-\frac{1}{2 \sqrt{\mu \lambda}}\right) f_{\mu}^{\prime}\left(b_{2}\right)<f_{\lambda}^{\prime}\left(\frac{1}{2}-\frac{1}{2 \sqrt[3]{\mu^{2} \lambda}}\right)\left(f_{\mu}^{\prime}\left(\frac{1}{2}-\frac{1}{2 \sqrt{\mu \lambda}}\right)\right)^{2}<1$,

and $f_{\lambda}^{\prime}\left(f_{\mu}\left(\frac{1}{2}-\frac{1}{2 \lambda}\right)\right) f_{\mu}^{\prime}\left(\frac{1}{2}-\frac{1}{2 \lambda}\right)<1$.

While if the process starting at $\xi \in\left[\frac{1}{2}-\frac{1}{2 \lambda}, 1-\frac{1}{\lambda}\right]$ enters $\left(\frac{1}{2}-\frac{1}{2 \sqrt[3]{\mu^{2} \lambda}}, f_{\mu}\left(\frac{1}{2}-\frac{1}{2 \sqrt{\mu \lambda}}\right)\right)$ and then comes back to $\left[\frac{1}{2}-\frac{1}{2 \lambda}, 1-\frac{1}{\lambda}\right]$, by Lemma 2.1.12 and Lemma 2.1.14, in the sequence, the number of $f_{\mu}^{\prime} s$ is at least twice as many as the number of $f_{\lambda}^{\prime} s$ and the largest derivative is $f_{\lambda}^{\prime}\left(\frac{1}{2}-\frac{1}{2 \sqrt[3]{\mu^{2} \lambda}}\right)\left(f_{\mu}^{\prime}\left(d_{3}\right)\right)^{2}$.

(b) If the process will stay in $\left(\frac{1}{2}-\frac{1}{2 \sqrt[3]{\mu^{2} \lambda}}, \frac{1}{2}-\frac{1}{2 \sqrt{\mu \lambda}}\right]$ forever. By Lemma 2.1.12 and Lemma 2.1.14, in the sequence, the number of $f_{\mu}^{\prime} s$ is at least twice as many as the number of $f_{\lambda}^{\prime} s$. But any two $f_{\mu}^{\prime} s$ and one $f_{\lambda}^{\prime} s$ makes the product of the absolute value of their derivatives less than 1 and all of them are controlled by $f_{\lambda}^{\prime}\left(\frac{1}{2}-\frac{1}{2 \sqrt[3]{\mu^{2} \lambda}}\right)\left(f_{\mu}^{\prime}\left(d_{3}\right)\right)^{2}<1$.

In all cases of $(a)$ and $(b)$, one can easily get the following estimate

$$
\left|\frac{d}{d \xi}\left[f_{\epsilon_{-1}} f_{\epsilon_{-2}} \cdots f_{\epsilon_{-n}}(\xi)\right]\right| \leqslant L_{3}^{\left[\frac{n}{3}\right]},
$$


where

$$
\begin{aligned}
L_{3}= & \max \left\{f_{\lambda}^{\prime}\left(f_{\mu}\left(\frac{1}{2}-\frac{1}{2 \lambda}\right)\right) f_{\mu}^{\prime}\left(\frac{1}{2}-\frac{1}{2 \lambda}\right), f_{\lambda}^{\prime}\left(f_{\mu}\left(\frac{1}{2}-\frac{1}{2 \sqrt{\mu \lambda}}\right)\right) f_{\mu}^{\prime}\left(\frac{1}{2}-\frac{1}{2 \sqrt{\mu \lambda}}\right) f_{\mu}^{\prime}\left(b_{2}\right),\right. \\
& \left.f_{\lambda}^{\prime}\left(\frac{1}{2}-\frac{1}{2 \sqrt[3]{\mu^{2} \lambda}}\right)\left(f_{\mu}^{\prime}\left(d_{3}\right)\right)^{2}, L_{2}\right\}<1
\end{aligned}
$$

(iv) Starting from $\xi \in\left[\frac{1}{2}-\frac{1}{2 \lambda}, 1-\frac{1}{\lambda}\right]$, if the process is mapped by $f_{\mu}$ into the interval $\left(\frac{1}{2}-\frac{1}{2 \sqrt[4]{\mu^{3} \lambda}}, \frac{1}{2}-\frac{1}{2 \sqrt[3]{\mu^{2} \lambda}}\right]$, then consider cases

(a) The process will stay in $\left(\frac{1}{2}-\frac{1}{2 \sqrt[4]{\mu^{3} \lambda}}, \frac{1}{2}-\frac{1}{2 \sqrt[3]{\mu^{2} \lambda}}\right]$ forever. By Lemma 2.1.13 and Lemma 2.1.14, then the process from the time that the process enters $\left(\frac{1}{2}-\frac{1}{2 \sqrt[4]{\mu^{3} \lambda}}, \frac{1}{2}-\frac{1}{2 \sqrt[3]{\mu^{2} \lambda}}\right]$, the number of $f_{\mu}^{\prime} s$ is at least three times as many as the number of $f_{\lambda}^{\prime} s$ and the multiplication of the derivatives is controlled by $f_{\lambda}^{\prime}\left(\frac{1}{2}-\frac{1}{2 \sqrt[4]{\mu^{3} \lambda}}\right) f_{\mu}^{\prime}\left(d_{3}\right)^{3}<1$.

(b) The process will come back to the interval $\left(\frac{1}{2}-\frac{1}{2 \sqrt[3]{\mu^{2} \lambda}}, \frac{1}{2}-\frac{1}{2 \sqrt{\mu \lambda}}\right]$. With the same reason as (I)(3)(b) when $1<\mu<\lambda \leqslant 2$, by Lemma 2.1 .13 , the multiplication of the derivatives is controlled by $f_{\lambda}^{\prime}\left(\frac{1}{2}-\frac{1}{2 \sqrt[4]{\mu^{3} \lambda}}\right) f_{\mu}^{\prime}\left(d_{3}\right)^{3}<1$. After that it will be case (iii) again.

If the process enters $\left(\frac{1}{2}-\frac{1}{2 \sqrt{\mu \lambda}}, \frac{1}{2}-\frac{1}{2 \lambda}\right)$ after it enters $\left(\frac{1}{2}-\frac{1}{2 \sqrt[3]{\mu^{2} \lambda}}, \frac{1}{2}-\frac{1}{2 \sqrt{\mu \lambda}}\right]$, then by Lemma 2.1.13, 2.1.14 and Lemma 2.1.12, in the process from the time that the process enters $\left(\frac{1}{2}-\frac{1}{2 \sqrt[4]{\mu^{3} \lambda}}, \frac{1}{2}-\frac{1}{2 \sqrt[3]{\mu^{2} \lambda}}\right]$ to the first time it goes to $\left(\frac{1}{2}-\right.$ $\left.\frac{1}{2 \sqrt{\mu \lambda}}, \frac{1}{2}-\frac{1}{2 \lambda}\right)$, the multiplication of the derivatives is controlled by $\max \left\{f_{\lambda}^{\prime}\left(\frac{1}{2}-\right.\right.$ $\left.\left.\frac{1}{2 \sqrt[4]{\mu^{3} \lambda}}\right) f_{\mu}^{\prime}\left(d_{3}\right)^{3}, f_{\lambda}^{\prime}\left(f_{\mu}\left(\frac{1}{2}-\frac{1}{2 \sqrt[3]{\mu^{2} \lambda}}\right)\right) f_{\mu}^{\prime}\left(\frac{1}{2}-\frac{1}{2 \sqrt[3]{\mu^{2} \lambda}}\right) f_{\mu}^{\prime}\left(d_{3}\right)\right\}$

If the process enters $\left[\frac{1}{2}-\frac{1}{2 \lambda}, 1-\frac{1}{\lambda}\right]$ after it enters $\left(\frac{1}{2}-\frac{1}{2 \sqrt{\mu \lambda}}, \frac{1}{2}-\frac{1}{2 \lambda}\right)$, then by Lemma 2.1.13, 2.1.14, 2.1.12 and Lemma 2.1.2, in the process, from the time that the process enters $\left(\frac{1}{2}-\frac{1}{2 \sqrt[4]{\mu^{3} \lambda}}, \frac{1}{2}-\frac{1}{2 \sqrt[3]{\mu^{2} \lambda}}\right]$ to the first time it goes to $\left[\frac{1}{2}-\frac{1}{2 \lambda}, 1-\frac{1}{\lambda}\right]$, the multiplication of the derivatives is controlled by $\max \left\{f_{\lambda}^{\prime}\left(\frac{1}{2}-\right.\right.$ $\left.\left.\frac{1}{2 \sqrt[4]{\mu^{3} \lambda}}\right) f_{\mu}^{\prime}\left(d_{3}\right)^{3}, f_{\lambda}^{\prime}\left(f_{\mu}\left(\frac{1}{2}-\frac{1}{2 \sqrt[3]{\mu^{2} \lambda}}\right)\right) f_{\mu}^{\prime}\left(\frac{1}{2}-\frac{1}{2 \sqrt[3]{\mu^{2} \lambda}}\right) f_{\mu}^{\prime}\left(d_{3}\right), L_{3}\right\}$

As a result, considering both cases of $(a)$ and $(b)$, one can easily get:

$$
\left|\frac{d}{d \xi}\left[f_{\epsilon_{-1}} f_{\epsilon_{-2}} \cdots f_{\epsilon_{-n}}(\xi)\right]\right| \leqslant L_{4}^{\left[\frac{n}{4}\right]}
$$

where

$L_{4}=\max \left\{f_{\lambda}^{\prime}\left(\frac{1}{2}-\frac{1}{2 \sqrt[4]{\mu^{3} \lambda}}\right) f_{\mu}^{\prime}\left(d_{3}\right)^{3}, f_{\lambda}^{\prime}\left(f_{\mu}\left(\frac{1}{2}-\frac{1}{2 \sqrt[3]{\mu^{2} \lambda}}\right)\right) f_{\mu}^{\prime}\left(\frac{1}{2}-\frac{1}{2 \sqrt[3]{\mu^{2} \lambda}}\right) f_{\mu}^{\prime}\left(d_{3}\right), L_{3}\right\}<1$.

(v) The above argument can continue. Let's say now the point from $\left[\frac{1}{2}-\frac{1}{2 \lambda}, 1-\frac{1}{\lambda}\right]$ is mapped to the interval $\left[1-\frac{1}{\mu}, \frac{1}{2}-\frac{1}{2 \sqrt[k]{\mu^{k-1} \lambda}}\right]$. There are two possibilities: 
(a) The process remains in $\left[1-\frac{1}{\mu}, \frac{1}{2}-\frac{1}{2 \sqrt[k]{\mu^{k-1} \lambda}}\right]$ forever. Then by Lemma 2.1.14 and Lemma 2.1.13, in the sequence from the time $x$ enters $\left[1-\frac{1}{\mu}, \frac{1}{2}-\frac{1}{2 \sqrt[k]{\mu^{k-1} \lambda}}\right]$, the number of $f_{\mu}^{\prime} s$ is at least $k$ times as many as the number of $f_{\lambda}^{\prime} s$. Again any $k f_{\mu}^{\prime} s$ and one $f_{\lambda}$ make the multiplication of their derivatives less than 1 , the largest of these is

$$
f_{\lambda}^{\prime}\left(1-\frac{1}{\mu}\right)\left(f_{\mu}^{\prime}\left(1-\frac{1}{\mu}\right)\right)^{k}<1 .
$$

(b) The process leaves $\left[1-\frac{1}{\mu}, \frac{1}{2}-\frac{1}{2 \sqrt[k]{\mu^{k-1} \lambda}}\right]$ and goes to $\left(\frac{1}{2}-\frac{1}{2 \sqrt[k]{\mu^{k-1} \lambda}}, \frac{1}{2}-\frac{1}{2 \sqrt[k-1]{\mu^{k-2} \lambda}}\right]$. For this case, as the description in the case (I)(4)(c) when $1<\mu<\lambda \leqslant 2$, the largest derivative of the multiplication is

$$
f_{\lambda}^{\prime}\left(1-\frac{1}{\mu}\right)\left(f_{\mu}^{\prime}\left(1-\frac{1}{\mu}\right)\right)^{k}<1
$$

After case (b), it will come to the previous cases.

Summarizing above, we can get

$$
\left|\frac{d}{d \xi}\left[f_{\epsilon_{-1}} f_{\epsilon_{-2}} \cdots f_{\epsilon_{-n}}(\xi)\right]\right| \leqslant L_{k+1}^{\left[\frac{n}{k+1}\right]}
$$

where

$$
\begin{aligned}
L_{k+1}= & \max \left\{f_{\lambda}^{\prime}\left(f_{\mu}\left(\frac{1}{2}-\frac{1}{2 \sqrt[k]{\mu^{k-1} \lambda}}\right)\right) f_{\mu}^{\prime}\left(\frac{1}{2}-\frac{1}{2 \sqrt[k]{\mu^{k-1} \lambda}}\right)\left(f_{\mu}^{\prime}\left(d_{k}\right)\right)^{k-2},\right. \\
& \left.f_{\lambda}^{\prime}\left(1-\frac{1}{\mu}\right)\left(f_{\mu}^{\prime}\left(1-\frac{1}{\mu}\right)\right)^{k}, L_{k}\right\}<1
\end{aligned}
$$

and $d_{k}$ is defined by $f_{\mu}\left(d_{k}\right)=\frac{1}{2}-\frac{1}{2 \sqrt[k]{\mu^{k-1} \lambda}}$ and

$$
\begin{aligned}
L_{k}= & \max \left\{f_{\lambda}^{\prime}\left(\frac{1}{2}-\frac{1}{2 \sqrt[k]{\mu^{k-1} \lambda}}\right)\left(f_{\mu}^{\prime}\left(d_{k}\right)\right)^{k-1}\right. \\
& \left.f_{\lambda}^{\prime}\left(f_{\mu}\left(\frac{1}{2}-\frac{1}{2 \sqrt[k-1]{\mu^{k-2} \lambda}}\right)\right) f_{\mu}^{\prime}\left(\frac{1}{2}-\frac{1}{2 \sqrt[k-1]{\mu^{k-2} \lambda}}\right)\left(f_{\mu}^{\prime}\left(d_{k-1}\right)\right)^{k-3}, L_{k-1}\right\}<1 .
\end{aligned}
$$

(II). $\xi \in\left(\frac{1}{2}-\frac{1}{2 \sqrt{\mu \lambda}}, \frac{1}{2}-\frac{1}{2 \lambda}\right)$.

Consider the following possibilities:

(i) If the process starting at $\xi \in\left(\frac{1}{2}-\frac{1}{2 \sqrt{\mu \lambda}}, \frac{1}{2}-\frac{1}{2 \lambda}\right)$ enters the interval $\left[\frac{1}{2}-\frac{1}{2 \lambda}, 1-\frac{1}{\lambda}\right]$, then by Lemma 2.1.15, $f_{\lambda}\left(\frac{1}{2}-\frac{1}{2 \sqrt{\mu \lambda}}\right)>\frac{1}{2}-\frac{1}{2 \lambda}$. So for any $\xi \in\left(\frac{1}{2}-\frac{1}{2 \sqrt{\mu \lambda}}, \frac{1}{2}-\frac{1}{2 \lambda}\right)$, we have

$$
\left|\frac{d}{d \xi}\left[f_{\epsilon_{-1}} f_{\epsilon_{-2}} \cdots f_{\epsilon_{-n}}(\xi)\right]\right| \leqslant M_{1} L_{k+1}^{\left[\frac{n-1}{k+1}\right]},
$$

where $M_{1}=f_{\lambda}^{\prime}\left(\frac{1}{2}-\frac{1}{2 \sqrt{\mu \lambda}}\right)$. 
(ii) The process starting at $\xi \in\left(\frac{1}{2}-\frac{1}{2 \sqrt{\mu \lambda}}, \frac{1}{2}-\frac{1}{2 \lambda}\right)$ stays in this interval forever, then (2.37) also holds as the case (I)(iii).

(iii) If $\xi \in\left(\frac{1}{2}-\frac{1}{2 \sqrt{\mu \lambda}}, \frac{1}{2}-\frac{1}{2 \lambda}\right)$ is mapped into the interval $\left(\frac{1}{2}-\frac{1}{2 \sqrt[3]{\mu^{2} \lambda}}, \frac{1}{2}-\frac{1}{2 \sqrt{\mu \lambda}}\right]$ and never to $\left[1-\frac{1}{\mu}, \frac{1}{2}-\frac{1}{2 \sqrt[3]{\mu^{2} \lambda}}\right]$. After that, if the process stays in that interval forever, then (2.38) holds. However, if the process can jump out of the interval $\left(\frac{1}{2}-\frac{1}{2 \sqrt[3]{\mu^{2} \lambda}}, \frac{1}{2}-\frac{1}{2 \sqrt{\mu \lambda}}\right]$ again by $f_{\lambda}$, then as the case (II)(3)(b) when $1<\mu<\lambda \leqslant 2$, if this case (II)(iii) repeats, then we have

$$
\left|\frac{d}{d \xi}\left[f_{\epsilon_{-1}} f_{\epsilon_{-2}} \cdots f_{\epsilon_{-n}}(\xi)\right]\right| \leqslant N_{2} L_{3}^{\left[\frac{n-2}{k+1}\right]},
$$

where $N_{2}$ and $L_{3}$ are defined in the same way as in (2.14) and (2.38) respectively.

(iv) The above argument can continue. So if $\xi \in\left(\frac{1}{2}-\frac{1}{2 \sqrt{\mu \lambda}}, \frac{1}{2}-\frac{1}{2 \lambda}\right)$ is mapped into the interval $\left[1-\frac{1}{\mu}, \frac{1}{2}-\frac{1}{2 \sqrt[k]{\mu^{k-1} \lambda}}\right]$ at a point $\xi_{1}$. After that, if it stays in it forever, then (2.40) holds. While it is mapped by $f_{\lambda}$ to the interval $\left[1-\frac{1}{\mu}, \frac{1}{2}-\frac{1}{2 \sqrt[k]{\mu^{k-1} \lambda}}\right]$, it is the same case as $(\mathrm{I})(4)(\mathrm{b})$ when $1<\mu<\lambda \leqslant 2$. We define $\xi_{1}^{\prime}$ as $\underbrace{f_{\mu} \cdots f_{\mu}}_{k}\left(\xi_{1}^{\prime}\right)=\xi_{1}$, then by Lemma 2.1.2, Lemma 2.1.14 and Lemma 2.1.13, $f_{\mu}\left(\xi_{1}^{\prime}\right)<\frac{1}{2}-\frac{1}{2 \sqrt{\mu \lambda}}$ and $f_{\lambda}\left(\xi_{1}\right)>f_{\mu}\left(\xi_{1}^{\prime}\right)$. So we have the following inequality

$$
\left|\frac{d}{d \xi}\left[f_{\epsilon_{-1}} f_{\epsilon_{-2}} \cdots f_{\epsilon_{-n}}(\xi)\right]\right| \leqslant L_{k+1}^{\left[\frac{n}{k+1}\right]}
$$

where $L_{k+1}$ are defined the same as in (2.40). Concluding these four possibilities of case (II), for any $\xi \in\left(\frac{1}{2}-\frac{1}{2 \sqrt{\mu \lambda}}, \frac{1}{2}-\frac{1}{2 \lambda}\right)$, we always have

$$
\left|\frac{d}{d \xi}\left[f_{\epsilon_{-1}} f_{\epsilon_{-2}} \cdots f_{\epsilon_{-n}}(\xi)\right]\right| \leqslant M_{1} N_{2} L_{k+1}^{\left[\frac{n-3}{k+1}\right]} .
$$

We can discuss the cases $\xi \in\left(\frac{1}{2}-\frac{1}{2 \sqrt[3]{\mu^{2} \lambda}}, \frac{1}{2}-\frac{1}{2 \sqrt{\mu \lambda}}\right), \cdots, \xi \in\left[\frac{1}{2}-\frac{1}{2 \sqrt[k]{\mu^{k-1} \lambda}}, \frac{1}{2}-\frac{1}{2 \sqrt[k-1]{\mu^{k-2} \lambda}}\right]$ similarly, but we omit the details here.

(III). We consider the case $\xi \in\left[1-\frac{1}{\mu}, \frac{1}{2}-\frac{1}{2 \sqrt[k]{\mu^{k-1} \lambda}}\right]$.

(i) If $\xi \in\left[1-\frac{1}{\mu}, \frac{1}{2}-\frac{1}{2 \sqrt[k]{\mu^{k-1} \lambda}}\right]$ and the process stays in this interval forever, then (2.40) holds.

(ii) If the process moves to the right and eventually enters the interval $\left[\frac{1}{2}-\frac{1}{2 \lambda}, 1-\right.$ $\left.\frac{1}{\lambda}\right]$. During this process, when the process enters $\left[\frac{1}{2}-\frac{1}{2 \sqrt[i]{\mu^{i-1} \lambda}}, \frac{1}{2}-\frac{1}{2 \sqrt[i-1]{\mu^{i-2} \lambda}}\right)$, the following (case $\star$ ) is possible to happen for each $i=2,3, \cdots, k$. While for $i=2$, we have discussed in case (II)(iii), now we will consider other $i$. 
(Case $\star$ ) the process is mapped into the interval $\left[\frac{1}{2}-\frac{1}{2 \sqrt[i+1]{\mu^{i} \lambda}}, \frac{1}{2}-\frac{1}{2 \sqrt[i]{\mu^{i-1} \lambda}}\right)$ and then comes back to the interval $\left[\frac{1}{2}-\frac{1}{2 \sqrt[i]{\mu^{i-1} \lambda}}, \frac{1}{2}-\frac{1}{2 \sqrt[i-1]{\mu^{i-2} \lambda}}\right)$ with a map $f_{\lambda}$. Actually, we have discussed similar case in the case (IV) 3 under the condition $1<\mu<\lambda \leqslant 2$. By Lemma 2.1.13 and Lemma 2.1.14, the largest derivative of the multiplication is $\frac{d}{d x}\left[f_{\lambda}(\underbrace{f_{\mu} \cdots f_{\mu}}_{j-1}\left(\frac{1}{2}-\frac{1}{2 \sqrt[i]{\mu^{i-1} \lambda}}\right))\right]:=N_{i j}^{\prime} ;$ here $j=2,3, \cdots, i$.

Now it follows from Lemma 2.1.15 that

$$
\left|\frac{d}{d \xi}\left[f_{\epsilon_{-1}} f_{\epsilon_{-2}} \cdots f_{\epsilon_{-n}}(\xi)\right]\right| \leqslant \prod_{t=1}^{k} M_{t} \prod_{i=2}^{k} M_{i}^{\prime} L_{k+1}^{\left[\frac{n-k-\bar{k}}{k+1}\right]},
$$

where $M_{k}=f_{\lambda}^{\prime}\left(1-\frac{1}{\mu}\right) M_{t}=f_{\lambda}^{\prime}\left(\frac{1}{2}-\frac{1}{2 \sqrt[t]{\mu^{t-1} \lambda}}\right)$ for $t=1,2, \cdots, k-1, M_{i}^{\prime}=\prod_{j=2}^{i} N_{i j}^{\prime}$ for $i=2, \cdots, k$ and $\bar{k}=\sum_{m=2}^{k}\left(\sum_{i=2}^{m} i\right)$.

Concluding all above cases (I), (II) and (III), we can always get (2.45) for any $\xi \in[1-$ $\left.\frac{1}{\mu}, 1-\frac{1}{\lambda}\right]$ when $1<\mu \leqslant \frac{4}{\lambda}, 2<\lambda \leqslant 3$.

As a result, when $1<\mu \leqslant 2<\lambda \leqslant 3$, for any $x \in\left[1-\frac{1}{\mu}, 1-\frac{1}{\lambda}\right]$, using the same argument as in the proof of Proposition 2.1.6 when $1<\mu<\lambda \leqslant 2$, there is a limit of $f_{\epsilon_{-1}} f_{\epsilon_{-2}} \cdots f_{\epsilon_{-n}}(x)$, denoted by $Y^{\star}(\omega)$. It is also noted that $Y^{\star}(\omega)$ is independent of $x$. \#

\section{$2.23<\mu<\lambda \leqslant 1+\sqrt{5}$}

\subsubsection{Limit of pullback.}

Lemma 2.2.1 For $3.00547 \leqslant \mu<\lambda \leqslant 1+\sqrt{5}$ and $k \geqslant 2$, we have the following inequality:

$$
F_{\mu}\left(x_{k-1}\right)>x_{k},
$$

where $x_{k}$ satisfies $F_{\lambda}^{\prime}\left(x_{k}\right)\left(F_{\mu}^{\prime}\left(x_{k}\right)\right)^{k-1}=1$.

Let $F_{\theta}=f_{\theta}\left(f_{\theta}(x)\right)=\theta^{2} x(1-x)\left(1-\theta x+\theta x^{2}\right)$. Here, the reason we choose $\mu \geqslant 3.00547$ is that we need to guarantee $\beta_{\mu} \geqslant 1-\frac{1}{\lambda} \geqslant 1-\frac{1}{1+\sqrt{5}}$ in order to make sure that $\left[\beta_{\mu}, \beta_{\lambda}\right]$ is an invariant interval. Under this condition, $\left[\alpha_{\mu}, \alpha_{\lambda}\right]$ is also an invariant interval.

Lemma 2.2.2 Assume $F_{\lambda}^{\prime}\left(\beta_{\mu}\right) F_{\mu}^{\prime}\left(\beta_{\mu}\right)<1$, then for any $x \in\left[\beta_{\mu}, x_{1}\right]$, we have

$$
F_{\lambda}\left(F_{\mu}(x)\right)>x .
$$

Lemma 2.2.3 Assume $F_{\lambda}^{\prime}\left(\beta_{\mu}\right)\left(F_{\mu}^{\prime}\left(\beta_{\mu}\right)\right)^{2}<1$ and $3.00547 \leqslant \mu<\lambda \leqslant 1+\sqrt{5}$ and $y_{2}$ is defined by $F_{\mu}\left(F_{\mu}\left(y_{2}\right)\right)=x_{2}$. Then for any $x \in\left[\beta_{\mu}, y_{2}\right]$, we have

$$
F_{\lambda}\left(F_{\mu}\left(F_{\mu}(x)\right)\right)>x .
$$

Lemma 2.2.4 Assume $3.00547 \leqslant \mu<\lambda \leqslant 1+\sqrt{5}$, then we can always find a $k$ such that $x_{k+1}<\beta_{\mu} \leqslant x_{k}$, where $x_{k}$ is defined by $F_{\lambda}^{\prime}\left(x_{k}\right)\left(F_{\mu}^{\prime}\left(x_{k}\right)\right)^{k-1}=1$. Moreover, for any $x \in\left[\beta_{\mu}, y_{k}\right]$,

$$
F_{\lambda}(\underbrace{F_{\mu} \cdots F_{\mu}}_{k}(x))>x
$$


where $y_{k}$ is defined by $\underbrace{F_{\mu} \cdots F_{\mu}}_{k}\left(y_{k}\right)=x_{k}$.

Proposition 2.2.5 Assume $3.00547 \leqslant \mu<\lambda<1+\sqrt{5}$ and $\omega=\left(\cdots, \epsilon_{-2}, \epsilon_{-1} ; \epsilon_{0}, \epsilon_{1}, \cdots\right)$ with $\epsilon_{2 i}=\epsilon_{2 i+1}$ for all $i$, there exists $\alpha(\omega), \beta(\omega)$ such that for any $x \in\left[\beta_{\mu}, \beta_{\lambda}\right]$ and $y \in\left[\alpha_{\lambda}, \alpha_{\mu}\right]$, we can get

$$
\begin{aligned}
& \lim _{n \rightarrow \infty} f_{\epsilon_{-1}} \cdots f_{\epsilon_{-2 n}}(x)=\beta(\omega), \\
& \lim _{n \rightarrow \infty} f_{\epsilon_{-1}} \cdots f_{\epsilon_{-2 n}}(y)=\alpha(\omega) .
\end{aligned}
$$

Proof. $F_{\mu}$ and $F_{\lambda}$ are increasing in these two invariant intervals. For any $x \in(0,1)$, the process will enter either $\left[\alpha_{\lambda}, \alpha_{\mu}\right]$ or $\left[\beta_{\mu}, \beta_{\lambda}\right]$ after finite $F_{\mu}$ and finite $F_{\lambda}$.

If there are infinitely many $\mu$ and finitely many $\lambda$ in $\left(\epsilon_{1} \epsilon_{2} \cdots\right)$, then obviously $\mathrm{x}$ will go towards the attracting point $\alpha_{\mu}$ or $\alpha_{\lambda}$ eventually. i.e.

$$
\lim _{k \rightarrow \infty} F_{\epsilon_{-1}} F_{\epsilon_{-2}} \cdots F_{\epsilon_{-k}}(x)=\left\{\begin{array}{l}
F_{\epsilon_{-1}} F_{\epsilon_{-2}} \cdots F_{\epsilon_{-m_{1}}}\left(\alpha_{\mu}\right) \\
F_{\epsilon_{-1}} F_{\epsilon_{-2}} \cdots F_{\epsilon_{-m_{1}}}\left(\beta_{\mu}\right) \quad \text { when } x \in\left[\alpha_{\lambda}, \alpha_{\mu}\right],
\end{array} \text { when } x \in\left[\beta_{\mu}, \beta_{\lambda}\right] .\right.
$$

Here $m_{1} \in \mathbb{Z}$ such that $\epsilon_{-j}=\mu$ for all $j>m_{1}$.

With the same reason, if there are infinitely many $\lambda$ and finitely many $\mu$ in $\left(\epsilon_{1} \epsilon_{2} \cdots\right)$, then

$$
\lim _{k \rightarrow \infty} F_{\epsilon_{-1}} F_{\epsilon_{-2}} \cdots F_{\epsilon_{-k}}(x)=\left\{\begin{array}{l}
F_{\epsilon_{-1}} F_{\epsilon_{-2}} \cdots F_{\epsilon_{-m_{2}}}\left(\alpha_{\lambda}\right) \\
F_{\epsilon_{-1}} F_{\epsilon_{-2}} \cdots F_{\epsilon_{-m_{2}}}\left(\beta_{\lambda}\right) \quad \text { when } x \in\left[\alpha_{\lambda}, \alpha_{\mu}\right],
\end{array}\right.
$$

Here $m_{2} \in \mathbb{Z}$ such that $\epsilon_{-j}=\lambda$ for all $j>m_{2}$.

However, If there are infinitely many $\mu$ and infinitely many $\lambda$ in $\left(\epsilon_{1} \epsilon_{2} \cdots\right)$, this case will be more complicated than the above one.

Note that $F_{\mu}^{\prime}(x) \leqslant 1$ for $x \in\left[\beta_{\mu}, \beta_{\lambda}\right]$. On the other hand, we can also find a point $x_{1}<\beta_{\mu}$ which satisfies $F_{\lambda}^{\prime}\left(x_{1}\right)=1$ when $F_{\lambda}^{\prime}\left(\beta_{\mu}\right)<1$. Obviously, for any $x \in\left[\beta_{\mu}, \beta_{\lambda}\right]$, $F_{\epsilon_{k}}^{\prime}(x)<1$ for any $\epsilon_{k}=\lambda$ or $\mu$. Then it is obvious that the following limit exists when $n$ approaches infinity.

$$
\lim _{n \rightarrow \infty} F_{\epsilon_{-1}} F_{\epsilon_{-2}} \cdots F_{\epsilon_{-n}}(x)=Y(\omega) .
$$

While when $F_{\lambda}^{\prime}\left(\beta_{\mu}\right) \geqslant 1$, we will consider $F_{\lambda}^{\prime}\left(\beta_{\mu}\right)\left(F_{\mu}^{\prime}\left(\beta_{\mu}\right)\right)_{k}, k \geqslant 1$ respectively. Then we will use the following lemmas 2.2.1, 2.2.2, 2.2.3 and 2.2.4 to prove this proposition as Figure 2.13 shows.

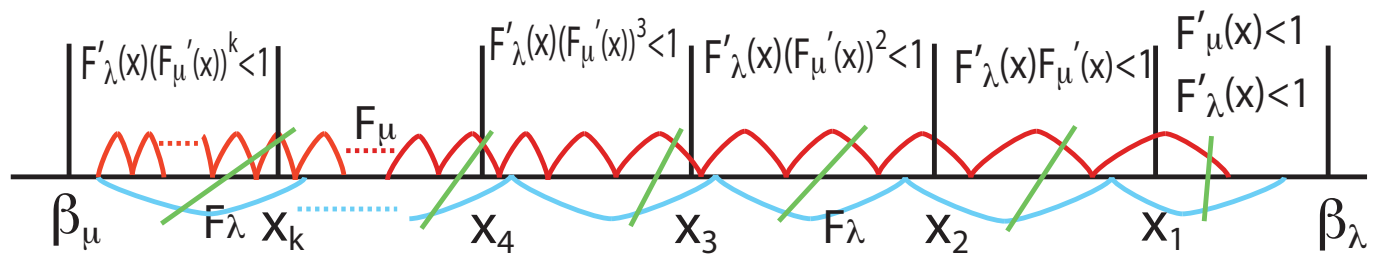

Figure 2.13: Contracted process 
Consider

$$
\omega=\left(\cdots, \epsilon_{-2}, \epsilon_{-1} ; \epsilon_{0}, \epsilon_{1}, \cdots\right),
$$

with $\epsilon_{2 i}=\epsilon_{2 i+1}, i=0, \pm 1, \pm 2, \cdots$. As Figure 2.13 shows, with the same reason as the case $1<\mu<\lambda \leqslant 2$. We have the following inequality for any $x, y \in\left[\beta_{\mu}, \beta_{\lambda}\right]$,

$$
\begin{aligned}
& \left|f_{\epsilon_{-1}} f_{\epsilon_{-2}} \cdots f_{\epsilon_{-(2 n-1)}} f_{\epsilon_{-2 n}}(x)-f_{\epsilon_{-1}} f_{\epsilon_{-2}} \cdots f_{\epsilon_{-(2 n-1)}} f_{\epsilon_{-2 n}}(y)\right| \\
& \leqslant M_{k}^{m_{k}} \prod_{j=2}^{k} M_{j}^{\prime} L_{k+1}^{\left[\frac{2 n-m_{k}-\tilde{m}}{k+1}\right]}|x-y| .
\end{aligned}
$$

Here the parameters are defined similarly as (2.23). $M_{k}=F_{\lambda}^{\prime}\left(\beta_{\mu}\right), M_{j}^{\prime}=\prod_{i=2}^{k} N_{j i}, N_{j i}=$ $\frac{d}{d x}\left[f_{\lambda}(\underbrace{f_{\mu} \cdots f_{\mu}}_{i-1}\left(x_{j}\right))\right], L_{k+1}=\max \left\{F_{\lambda}^{\prime}\left(\beta_{\mu}\right)\left(F_{\mu}^{\prime}\left(\beta_{\mu}\right)\right)^{k}, L_{k}\right\}, L_{k}=\max \left\{F_{\lambda}^{\prime}\left(x_{k}\right)\left(F_{\mu}^{\prime}(\underbrace{F_{\mu} \cdots F_{\mu}}_{k-1}\left(a_{k}\right))\right)^{k-1}\right\}$, $\tilde{m}=\sum_{m=2}^{k}\left(\sum_{i=2}^{k} i\right)$ and $m_{k}=n_{1}+\cdots+n_{i}+\cdots+n_{k}, n_{i}$ denotes the minimum number of $F_{\lambda}^{\prime} s$ in the following sequence

$$
F_{\lambda}(\underbrace{F_{\mu} \cdots F_{\mu}}_{i-1} F_{\lambda} \cdots \underbrace{F_{\mu} \cdots F_{\mu}}_{i-1} F_{\lambda}\left(x_{i+1}\right))>x_{i}, i=2, \cdots, k-1 .
$$

Moreover, $n_{k}$ denotes the minimum number of $F_{\lambda}$ in the following sequence

$$
F_{\lambda}(\underbrace{F_{\mu} \cdots F_{\mu}}_{k-1} F_{\lambda} \cdots \underbrace{F_{\mu} \cdots F_{\mu}}_{k-1} F_{\lambda}\left(\beta_{\mu}\right))>x_{k}
$$

If we choose $x=\beta_{\lambda}$ and $y=f_{\epsilon_{-(2 n+1)}} \cdots f_{\epsilon_{-2(n+m)}}\left(\beta_{\lambda}\right)$, by $(2.52)$, we can get as $n \rightarrow \infty$

$$
\begin{aligned}
& \left|f_{\epsilon_{-1}} f_{\epsilon_{-2}} \cdots f_{\epsilon_{-2 n}}\left(\beta_{\lambda}\right)-f_{\epsilon_{-1}} f_{\epsilon_{-2}} \cdots f_{\epsilon_{-2 n}}\left(f_{\epsilon_{-(2 n+1)}} \cdots f_{\epsilon_{-2(n+m)}}\left(\beta_{\lambda}\right)\right)\right| \\
& \leqslant M_{k}^{m_{k}} \prod_{j=2}^{k} M_{j}^{\prime} L_{k+1}^{\left[\frac{2 n-m_{k}-\tilde{m}}{k+1}\right]}\left|\beta_{\lambda}-f_{\epsilon_{-(2 n+1)}} \cdots f_{\epsilon_{-2(n+m)}}\left(\beta_{\lambda}\right)\right| \rightarrow 0 .
\end{aligned}
$$

As a result, $f_{\epsilon_{-1}} f_{\epsilon_{-2}} \cdots f_{\epsilon_{-n}}\left(\beta_{\lambda}\right)$ is a Cauchy sequence in $\left[\beta_{\mu}, \beta_{\lambda}\right]$. So there is a limit denoted by $\beta(\omega)$. Also for

$$
\begin{aligned}
\left|f_{\epsilon_{-1}} f_{\epsilon_{-2}} \cdots f_{\epsilon_{-2 n}}(x)-\beta(\omega)\right| \leqslant & \left|f_{\epsilon_{-1}} f_{\epsilon_{-2}} \cdots f_{\epsilon_{-2 n}}(x)-f_{\epsilon_{-1}} f_{\epsilon_{-2}} \cdots f_{\epsilon_{-2 n}}\left(\beta_{\lambda}\right)\right| \\
& +\left|f_{\epsilon_{-1}} f_{\epsilon_{-2}} \cdots f_{\epsilon_{-2 n}}\left(\beta_{\lambda}\right)-\beta(\omega)\right| \rightarrow 0 .
\end{aligned}
$$

Therefore, we can get

$$
\lim _{n \rightarrow \infty} f_{\epsilon_{-1}} f_{\epsilon_{-2}} \cdots f_{\epsilon_{-2 n}}(x)=\beta(\omega)
$$

which is independent of $x$.

With the same reason, for any $y \in\left[\alpha_{\lambda}, \alpha_{\mu}\right]$, we can get

$$
\lim _{n \rightarrow \infty} f_{\epsilon_{-1}} f_{\epsilon_{-2}} \cdots f_{\epsilon_{-2 n}}(y)=\alpha(\omega)
$$




\section{Chapter 3}

\section{Proof of the two main theorems}

\subsection{Proof of Theorem 1.2.2}

For $\omega=\left(\cdots, \epsilon_{-2}, \epsilon_{-1} ; \epsilon_{0}, \epsilon_{1}, \epsilon_{2}, \cdots, \epsilon_{k_{0}} \cdots\right)$, we define

$$
\begin{aligned}
\theta \omega & =\left(\cdots, \epsilon_{-2}, \epsilon_{-1}, \epsilon_{0} ; \epsilon_{1}, \epsilon_{2}, \cdots, \epsilon_{k_{0}} \cdots\right), \\
\theta^{-1} \omega & =\left(\cdots, \epsilon_{-2} ; \epsilon_{-1}, \epsilon_{0}, \epsilon_{1}, \epsilon_{2}, \cdots, \epsilon_{k_{0}} \cdots\right) .
\end{aligned}
$$

So $\theta^{n} \omega=\left(\cdots, \epsilon_{-2}, \epsilon_{-1}, \epsilon_{0}, \epsilon_{1}, \epsilon_{2} \cdots, \epsilon_{n-1} ; \epsilon_{n}, \cdots \epsilon_{k_{0}} \cdots\right)$.

Moreover, according to Proposition 2.1.6, Proposition 2.1.8 and Proposition 2.1.16, when $1<\mu<\lambda \leqslant 3$ for $x \in\left[1-\frac{1}{\mu}, 1-\frac{1}{\lambda}\right]$, we define

$$
Y^{\star}(\omega)=\lim _{n \rightarrow \infty} f_{\epsilon_{-1}} f_{\epsilon_{-2}} \cdots f_{\epsilon_{-(n-1)}} f_{\epsilon_{-n}}(x) .
$$

Recalling that we have defined $\Phi(\omega, x)=f_{\epsilon_{0}}(x)$, and it is easy to see that

$$
\begin{aligned}
\Phi_{2}(\omega, x) & =\Phi(\theta \omega) \Phi(\omega, x)=f_{\epsilon_{1}} f_{\epsilon_{0}}(x), \\
\Phi_{3}(\omega, x) & =\Phi\left(\theta^{2} \omega\right) \Phi(\theta \omega) \Phi(\omega, x)=f_{\epsilon_{2}} f_{\epsilon_{1}} f_{\epsilon_{0}}(x), \\
& \vdots \\
\Phi_{k}(\omega, x) & =\Phi\left(\theta^{k-1} \omega\right) \Phi\left(\theta^{k-2} \omega\right) \cdots \Phi(\theta \omega) \Phi(\omega, x) \\
& =f_{\epsilon_{k-1}} f_{\epsilon_{k-2}} \cdots f_{\epsilon_{1}} f_{\epsilon_{0}}(x) .
\end{aligned}
$$

So we can get

$$
\begin{aligned}
\Phi_{k}\left(\omega, Y^{\star}(\omega)\right) & =f_{\epsilon_{k-1}} f_{\epsilon_{k-2}} \cdots f_{\epsilon_{1}} f_{\epsilon_{0}} \lim _{n \rightarrow \infty} f_{\epsilon_{-1}} f_{\epsilon_{-2}} \cdots f_{\epsilon_{-(n-1)}} f_{\epsilon_{-n}}(x) \\
& =\lim _{n \rightarrow \infty} f_{\epsilon_{k-1}} f_{\epsilon_{k-2}} \cdots f_{\epsilon_{1}} f_{\epsilon_{0}} f_{\epsilon_{-1}} f_{\epsilon_{-2}} \cdots f_{\epsilon_{-(n-1)}} f_{\epsilon_{-n}}(x) \\
& =Y^{\star}\left(\theta^{k} \omega\right) .
\end{aligned}
$$

Therefore, $Y^{\star}(\omega)$ is a stationary solution of the random dynamical system.

Moreover

$$
\begin{aligned}
\Phi_{n}(\omega) & =\Phi\left(\theta^{n-1} \omega\right) \circ \Phi\left(\theta^{n-2} \omega\right) \circ \cdots \circ \Phi(\theta \omega) \circ \Phi(\omega), \\
\Phi_{n}\left(\theta^{-n} \omega\right)(x) & =\Phi\left(\theta^{-1} \omega\right) \circ \Phi\left(\theta^{-2} \omega\right) \circ \cdots \circ \Phi\left(\theta^{-n+1} \omega\right) \circ \Phi\left(\theta^{-n} \omega\right)(x)
\end{aligned}
$$




$$
=f_{\epsilon_{-1}} f_{\epsilon_{-2}} \cdots f_{\epsilon_{-(n-1)}} f_{\epsilon_{-n}}(x) .
$$

So for $x \in\left[1-\frac{1}{\mu}, 1-\frac{1}{\lambda}\right]$, we can get

$$
\lim _{n \rightarrow \infty} \Phi_{n}\left(\theta^{-n} \omega\right)(x)=\lim _{n \rightarrow \infty} f_{\epsilon_{-1}} f_{\epsilon_{-2}} \cdots f_{\epsilon_{-(n-1)}} f_{\epsilon_{-n}}(x)=Y^{\star}(\omega) .
$$

\subsection{Proof of Theorem 1.3.2}

With the same reason as the proof of theorem 1.2.2, and according to Proposition 2.2.5, we can get (1.9) and (1.10).

we define

$$
\alpha(\theta \omega)=f_{\epsilon_{0}}(\beta(\omega)), \quad \beta(\theta \omega)=f_{\epsilon_{0}}(\alpha(\omega)) .
$$

Then we have

$$
\begin{aligned}
& \Phi_{1}(\omega, \alpha(\omega))=f_{\epsilon_{0}}(\alpha(\omega))=\beta(\theta(\omega)), \\
& \Phi_{1}(\omega, \beta(\omega))=f_{\epsilon_{0}}(\beta(\omega))=\alpha(\theta(\omega)) .
\end{aligned}
$$

Consequently, by the cocycle property of random dynamical systems, we can get (1.11) and (1.12). By Definition 1.3.1, then Theorem 1.3.2 is proved. 


\section{Chapter 4}

\section{Proof of lemmas}

\subsection{Two general lemmas for $\mu, \lambda \in(1,3]$}

Proof of Lemma 2.1.2. To see the inequality (2.1) for $1<\mu<\lambda \leqslant 2$, define $m$ by $f_{\mu}(m)=\frac{1}{2}-\frac{1}{2 \sqrt[k+1]{\mu^{k} \lambda}}$. Then we need to prove that $m<\frac{1}{2}-\frac{1}{2 \sqrt[k]{\mu^{k-1} \lambda}}$, i.e.

$$
\frac{1}{2}-\frac{\sqrt{\mu^{2}-2 \mu+2 \mu^{\frac{1}{k+1}} \lambda^{-\frac{1}{k+1}}}}{2 \mu}<\frac{1}{2}-\frac{1}{2 \sqrt[k]{\mu^{k-1} \lambda}}
$$

It is equivalent to prove that $D(k)=\mu^{2}-2 \mu+2\left(\frac{\mu}{\lambda}\right)^{\frac{1}{k+1}}-\left(\frac{\mu}{\lambda}\right)^{\frac{2}{k}}>0$. Differentiating $D$ with respect to $k$, we have

$$
D^{\prime}(k)=\frac{2}{k^{2}} \ln \left(\frac{\mu}{\lambda}\right)\left(\frac{\mu}{\lambda}\right)^{\frac{1}{k+1}}\left[\left(\frac{\mu}{\lambda}\right)^{\frac{k+2}{k(k+1)}}-\frac{k^{2}}{(k+1)^{2}}\right] .
$$

Now, we will prove that $D^{\prime}(k)<0$. Since $\ln \left(\frac{\mu}{\lambda}\right)<0$, we need to prove that

$$
\left(\frac{\mu}{\lambda}\right)^{\frac{k+2}{k(k+1)}}-\frac{k^{2}}{(k+1)^{2}}>0 .
$$

Because $\left(\frac{\mu}{\lambda}\right)^{\frac{k+2}{k(k+1)}}$ is increasing as $\left(\frac{\mu}{\lambda}\right)$ increases, so $\left(\frac{\mu}{\lambda}\right)^{\frac{k+2}{k(k+1)}}>\left(\frac{1}{2}\right)^{\frac{k+2}{k(k+1)}}$. We will check whether or not $\left[\left(\frac{1}{2}\right)^{\frac{k+2}{k(k+1)}}-\frac{k^{2}}{(k+1)^{2}}\right]>0$. Considering that $\frac{k+2}{k(k+1)} \leqslant \frac{1}{k}+\frac{1}{k+1}<\frac{2}{k}$, we can get $\left(\frac{1}{2}\right)^{\frac{k+2}{k(k+1)}}>\left(\frac{1}{2}\right)^{\frac{2}{k}}$. In order to prove $D_{k}^{\prime}<0$, we need to prove $\left(\left(\frac{1}{2}\right)^{\frac{1}{k}}\right)^{2}>\left(\frac{k}{k+1}\right)^{2}$. So we will check whether or not $2^{\frac{1}{k}}-\frac{k+1}{k}<0$. Let $B(a)=2^{a}-1-a, a \in(0,1]$. Then it is easy to see that

$$
B^{\prime \prime}(a)=2^{a}(\ln 2)^{2}>0 .
$$

Therefore, the maximum point of $B$ should be at the start point or the end point of $a$. But $B(0)=0, B(1)=0$. As a result, $B\left(\frac{1}{k}\right)<0$. So $D^{\prime}(k)<0$. So $D(k)$ decreases as $k$ increases and for any $k \geqslant 1, D(k)>\lim _{k \rightarrow \infty} D(k)=\mu^{2}-2 \mu+1>0$. Thus the inequality (2.1) holds. 
Now, we will prove inequality (2.1) still holds for $\lambda \in(2,3]$ and $k>1$. We will check whether or not $\left[\left(\frac{1}{3}\right)^{\frac{k+2}{k(k+1)}}-\frac{k^{2}}{(k+1)^{2}}\right]>0$. Let $E(k)=3^{\frac{k+2}{2 k(k+1)}}-\frac{k+1}{k}$, then we differentiate $E$ with respect to $k$ to get

$$
E^{\prime}(k)=\frac{1}{k^{2}}\left[3^{\frac{k+2}{2 k(k+1)}}(\ln 3) \frac{-k^{2}-4 k-2}{2(k+1)^{2}}+1\right] .
$$

Here, let $F(k)=\left[3^{\frac{k+2}{2 k(k+1)}}(\ln 3) \frac{-k^{2}-4 k-2}{2(k+1)^{2}}+1\right]$ and we have

$$
F^{\prime}(k)=3^{\frac{k+2}{2 k(k+1)}}(\ln 3)\left[\frac{\left(-k^{2}-4 k-2\right)^{2}}{4 k^{2}(k+1)^{4}}+\frac{k}{(k+1)^{3}}\right]>0 .
$$

Therefore, $F(k)$ increases in $k, \lim _{k \rightarrow \infty} F(k)>0$ and $F(2)<0$. This implies that there exists $k_{0}$, such that

$$
E^{\prime}(k) \leqslant 0 \text { for } k \in\left[2, k_{0}\right], \quad E^{\prime}(k)>0, \text { for } k \in\left(k_{0}, \infty\right) .
$$

and $E(k)$ decreases first and then increase. So $E(k)$ reaches the maximum value at $k=2$ and $k=\infty$ for $k \geqslant 2$ :

$$
\begin{aligned}
& E(2)=3^{\frac{1}{3}}-\frac{3}{2}<0, \\
& \lim _{k \rightarrow \infty} E(k)=0 .
\end{aligned}
$$

It is easy to see now $E(k)<0$ when $k \geqslant 2$ and Lemma 2.1.2 holds. When $k=1$,

$$
\begin{array}{ll} 
& f_{\lambda}^{\prime}\left(f_{\mu}\left(\frac{1}{2}-\frac{1}{2 \lambda}\right)\right) f_{\mu}^{\prime}\left(\frac{1}{2}-\frac{1}{2 \lambda}\right)=\frac{2 \lambda^{2} \mu-\lambda^{2} \mu^{2}+\mu^{2}}{2 \lambda^{2}}, \\
\text { and } \quad 2 \lambda^{2} \mu-\lambda^{2} \mu^{2}+\mu^{2}-2 \lambda^{2}=\lambda^{2}\left(2 \mu-\mu^{2}-2\right)+\mu^{2}
\end{array}
$$

When $\lambda^{2}>\frac{\mu^{2}}{2 \mu-\mu^{2}-2}, f_{\lambda}^{\prime}\left(f_{\mu}\left(\frac{1}{2}-\frac{1}{2 \lambda}\right)\right) f_{\mu}^{\prime}\left(\frac{1}{2}-\frac{1}{2 \lambda}\right)<1$. Because $\left(\frac{\mu^{2}}{\mu^{2}+2-2 \mu}\right)^{\prime}=\frac{2 \mu(2-\mu)}{\left(\mu^{2}+2-2 \mu\right)^{2}}>0$ and $\frac{\mu^{2}}{\mu^{2}+2-2 \mu}<2$, it is easy to get $\lambda^{2}>\frac{\mu^{2}}{2 \mu-\mu^{2}-2}$ and

$$
f_{\lambda}^{\prime}\left(f_{\mu}\left(\frac{1}{2}-\frac{1}{2 \lambda}\right)\right) f_{\mu}^{\prime}\left(\frac{1}{2}-\frac{1}{2 \lambda}\right)<1 .
$$

Proof of Lemma 2.1.15. In order to prove (2.33), we just need to prove

$$
\lambda \frac{\mu^{2}-\left(\frac{\mu}{\lambda}\right)^{\frac{2}{k}}}{4 \mu^{2}}>\frac{\mu-\left(\frac{\mu}{\lambda}\right)^{\frac{1}{k-1}}}{2 \mu} .
$$

We let $A(\mu, \lambda, k)=\lambda \mu^{2}-\lambda\left(\frac{\mu}{\lambda}\right)^{\frac{2}{k}}-2 \mu^{2}+2 \mu\left(\frac{\mu}{\lambda}\right)^{\frac{1}{k-1}}$ and we differentiate $A(\mu, \lambda, k)$ with respect to $\mu$ to get

$$
\begin{aligned}
\frac{\partial A(\mu, \lambda, k)}{\partial \mu} & =2 \lambda \mu-4 \mu+2\left(\frac{\mu}{\lambda}\right)^{\frac{1}{k-1}}+\frac{2}{k-1}\left(\frac{\mu}{\lambda}\right)^{\frac{1}{k-1}}-\frac{2 \mu}{k \lambda}\left(\frac{\mu}{\lambda}\right)^{\frac{2}{k}} \\
& >2\left[\frac{1}{k-1}\left(\frac{\mu}{\lambda}\right)^{\frac{1}{k-1}}+\left(\frac{\mu}{\lambda}\right)^{\frac{1}{k-1}}-\frac{3}{k \mu}\left(\frac{\mu}{\lambda}\right)^{\frac{2}{k}}\right] .
\end{aligned}
$$


Since $\lambda \in(2,3]$, we can get $\left(\frac{\mu}{\lambda}\right)^{\frac{1}{k-1}}>\left(\frac{\mu}{\lambda}\right)^{\frac{2}{k}}$ and $\left(\frac{\mu}{\lambda}\right)^{\frac{1}{k-1}}>\frac{3}{k \mu}\left(\frac{\mu}{\lambda}\right)^{\frac{2}{k}}$ when $k \geqslant 3$. However, when $k=2$, we have

$$
\frac{\partial A(\mu, \lambda, k)}{\partial \mu}>2\left[\frac{2 \mu}{\lambda}-\frac{3}{2 \lambda}\right]>0 .
$$

As a result, $\frac{\partial A(\mu, \lambda, k)}{\partial \mu}>0$ and $A(\mu, \lambda, k)$ increases as $\mu$ increases. So we can get

$$
A(\mu, \lambda, k)>A(1, \lambda, k)=\lambda-\lambda^{\frac{k-2}{k}}-2+2 \lambda^{-\frac{1}{k-1}} .
$$

When $k \geqslant 2$,

$$
\begin{aligned}
\frac{\partial A(1, \lambda, k)}{\partial \lambda} & =1-\lambda^{-\frac{2}{k}}+\frac{2}{k} \lambda^{-\frac{2}{k}}-\frac{2}{(k-1) \lambda} \lambda^{-\frac{1}{k-1}} \\
& >\frac{2}{k} \lambda^{-\frac{2}{k}}-\frac{1}{k-1} \lambda^{-\frac{1}{k-1}}=\frac{\lambda^{-\frac{2}{k}}}{k}\left(2-\frac{k}{k-1} \lambda^{\frac{k-2}{k(k-1)}}\right) .
\end{aligned}
$$

Since $\lambda^{\frac{k-2}{k(k-1)}} \leqslant 3^{\frac{1}{6}}=1.2$ and $\frac{k}{k-1} \leqslant \frac{3}{2}$ for $k \geqslant 3$, and we also have $2-\frac{k}{k-1} \lambda^{\frac{k-2}{k(k-1)}}=0$ when $k=2$. Therefore, $\frac{\partial A(1, \lambda, k)}{\partial \lambda}>0$ and $A(1, \lambda, k)$ is an increasing function with respect to $\lambda$. So we can get

$$
A(\mu, \lambda, k)>A(1,2, k)=2\left(2^{-\frac{1}{k-1}}-2^{-\frac{2}{k}}\right)>0 .
$$

As a result, (2.33) holds and Lemma 2.1.15 is proved.

\section{$4.21<\mu<\lambda \leqslant 2$}

\subsection{1 $\lambda \geqslant \frac{\mu}{2-\mu}$}

In the case $\frac{\mu}{2-\mu} \leqslant \lambda<\frac{\mu}{(2-\mu)^{2}}$, we have $\frac{1}{2}-\frac{1}{2 \sqrt{\mu \lambda}}<1-\frac{1}{\mu} \leqslant \frac{1}{2}-\frac{1}{2 \lambda}$ as Figure 2.2 shows. Recall that $f_{\mu}^{\prime}(x)$ and $f_{\lambda}^{\prime}(x)$ are both less than 1 in $\left(\frac{1}{2}-\frac{1}{2 \lambda}, 1-\frac{1}{\lambda}\right]$. However, for $x \in\left(\frac{1}{2}-\frac{1}{2 \sqrt{\mu \lambda}}, \frac{1}{2}-\frac{1}{2 \lambda}\right], f_{\mu}^{\prime}(x) f_{\lambda}^{\prime}(x)<$ and $f_{\lambda}^{\prime}(x) \geqslant 1$. We prove the following lemma.

Proof of Lemma 2.1.3. We first prove that (2.2) holds for $x=\frac{1}{2}-\frac{1}{2 \lambda}$. Let $F(x)=$ $f_{\lambda}\left(f_{\mu}(x)\right)-x$ for any $x \in\left[\frac{1}{2}-\frac{1}{2 \sqrt{\mu \lambda}}, 1-\frac{1}{\lambda}\right]$, then we have

$$
F(x)=x\left[\lambda \mu(1-x)\left(1-\mu x+\mu x^{2}\right)-1\right] .
$$

For this, let $G(x)=\lambda \mu(1-x)\left(1-\mu x+\mu x^{2}\right)-1$. Then

$$
G^{\prime}(x)=\lambda \mu\left(-3 \mu x^{2}+4 \mu x-\mu-1\right)=-3 \lambda \mu^{2}\left(x-\frac{2}{3}\right)^{2}+\mu \lambda\left(\frac{\mu}{3}-1\right)<0 .
$$

So $G(x)$ is decreasing with respect to $x, G(x) \geqslant G\left(\frac{1}{2}-\frac{1}{2 \lambda}\right)$ for any $x \in\left[1-\frac{1}{\mu}, \frac{1}{2}-\frac{1}{2 \lambda}\right]$. Simple computation shows that

$$
G\left(\frac{1}{2}-\frac{1}{2 \lambda}\right)=\frac{\mu(\lambda+1)\left(4 \lambda^{2}-\mu \lambda^{2}+\mu\right)-8 \lambda^{2}}{8 \lambda^{2}} .
$$


But

$$
\begin{aligned}
A(\mu, \lambda) & =\mu(\lambda+1)\left(4 \lambda^{2}-\mu \lambda^{2}+\mu\right)-8 \lambda^{2} \\
& =-(\lambda+1)\left(\lambda^{2}-1\right)\left(\mu-\frac{2 \lambda^{2}}{\lambda^{2}-1}\right)^{2}+\frac{4 \lambda^{4}}{\lambda-1}-8 \lambda^{2} .
\end{aligned}
$$

It is trivial to see that $\frac{2 \lambda^{2}}{\lambda^{2}-1}>2$ and so $\mu<\frac{2 \lambda^{2}}{\lambda^{2}-1}$. Therefore, $A(\mu, \lambda)$ is monotonically increasing when $\mu$ increases in the interval $(1,2)$.

But when $\mu=1, A(1, \lambda)=3 \lambda^{3}-5 \lambda^{2}+\lambda+1>0$ for $\lambda>1$. Therefore $A(\mu, \lambda)>$ $A(1, \lambda)>0$. So $G\left(\frac{1}{2}-\frac{1}{2 \lambda}\right)=\frac{A}{8 \lambda^{2}}>0$ and $F\left(\frac{1}{2}-\frac{1}{2 \lambda}\right)>0$. Consequently, (2.2) holds for $x=\frac{1}{2}-\frac{1}{2 \lambda}$.

On the other hand, for $x \in\left[1-\frac{1}{\mu}, \frac{1}{2}-\frac{1}{2 \lambda}\right], f_{\mu}^{\prime}(x)>0, f_{\lambda}^{\prime}(x)>0, f_{\mu}^{\prime \prime}(x)=-2 \mu<0$ and $f_{\lambda}^{\prime \prime}(x)=-2 \lambda<0$, so we have

$$
\begin{aligned}
& F^{\prime}(x)=f_{\lambda}^{\prime}\left(f_{\mu}(x)\right) \cdot f_{\mu}^{\prime}(x)-1, \\
& F^{\prime \prime}(x)=f_{\lambda}^{\prime \prime}\left(f_{\mu}(x)\right)\left(f_{\mu}^{\prime}\right)^{2}+f_{\lambda}^{\prime}\left(f_{\mu}(x)\right) \cdot f_{\mu}^{\prime \prime}(x)=-2 \lambda\left(f_{\mu}^{\prime}\right)^{2}-2 \mu f_{\lambda}^{\prime}\left(f_{\mu}(x)\right)<0
\end{aligned}
$$

As a result, $F(x)$ gets the minimum value when $x=1-\frac{1}{\mu}$ or $x=\frac{1}{2}-\frac{1}{2 \lambda}$. It is easy to see that $F\left(1-\frac{1}{\mu}\right)>0$ and we recall that $F\left(\frac{1}{2}-\frac{1}{2 \lambda}\right)>0$, so we can get $F(x)>0$ for $x \in\left[\frac{1}{2}-\frac{1}{2 \sqrt{\mu \lambda}}, \frac{1}{2}-\frac{1}{2 \lambda}\right]$. This lemma is proved.

For $x \in\left(\frac{1}{2}-\frac{1}{2 \lambda}, 1-\frac{1}{\lambda}\right)$, note $f_{\mu}(x)>f_{\mu}\left(\frac{1}{2}-\frac{1}{2 \lambda}\right)$ and $f_{\lambda}\left(f_{\mu}\left(\frac{1}{2}-\frac{1}{2 \lambda}\right)\right)>\frac{1}{2}-\frac{1}{2 \lambda}$. So for $x \in\left(\frac{1}{2}-\frac{1}{2 \lambda}, 1-\frac{1}{\lambda}\right)$, we have

$$
f_{\lambda}\left(f_{\mu}(x)\right)>f_{\lambda}\left(f_{\mu}\left(\frac{1}{2}-\frac{1}{2 \lambda}\right)\right)>\frac{1}{2}-\frac{1}{2 \lambda} .
$$

That is to say, when $x \in\left(\frac{1}{2}-\frac{1}{2 \lambda}, 1-\frac{1}{\lambda}\right)$, then $f_{\lambda}\left(f_{\mu}(x)\right) \in\left(\frac{1}{2}-\frac{1}{2 \lambda}, 1-\frac{1}{\lambda}\right)$.

\subsection{2 $\lambda \geqslant \frac{\mu}{(2-\mu)^{2}}$}

In this case, $\frac{1}{2}-\frac{1}{2 \sqrt{\mu \lambda}} \geqslant 1-\frac{1}{\mu}$ i.e. $\frac{\mu}{(2-\mu)^{2}} \leqslant \lambda$. Note $f_{\lambda}^{\prime}(x) f_{\mu}^{\prime}(x) \geqslant 1$ for any $x \in$ $\left[1-\frac{1}{\mu}, \frac{1}{2}-\frac{1}{2 \sqrt{\mu \lambda}}\right]$. So we can not claim that $\left|f_{\lambda}\left(f_{\mu}(x)\right)-f_{\lambda}\left(f_{\mu}(y)\right)\right|<|x-y|$ for any $x, y \in\left[1-\frac{1}{\mu}, \frac{1}{2}-\frac{1}{2 \sqrt{\mu \lambda}}\right]$ and then we can not guarantee the process mapped out of the interval $\left(\frac{1}{2}-\frac{1}{2 \lambda}, 1-\frac{1}{\lambda}\right]$ and then back to this interval is contracting by what we have proved so far.

Now we consider the process that $\mathrm{x}$ is mapped by $f_{\mu}$ twice and then by $f_{\lambda}$. First note $f_{\lambda}^{\prime}(x) f_{\mu}^{\prime}(x) f_{\mu}^{\prime}(x)=\lambda \mu^{2}(1-2 x)^{3} \leqslant 1$ when $x>\frac{1}{2}\left(1-\frac{1}{\sqrt[3]{\mu^{2} \lambda}}\right)$. Therefore, when $\frac{1}{2}(1-$ $\left.\frac{1}{\sqrt[3]{\mu^{2} \lambda}}\right)<1-\frac{1}{\mu}$, i.e $\lambda<\frac{\mu}{(2-\mu)^{3}}$, we have for any $x \in\left(1-\frac{1}{\mu}, \frac{1}{2}-\frac{1}{2 \sqrt{\mu \lambda}}\right], f_{\lambda}^{\prime}(x) f_{\mu}(x)^{\prime} f_{\mu}^{\prime}(x)<1$ and $\frac{d}{d x}\left[f_{\lambda}\left(f_{\mu} f_{\mu}(x)\right)\right]<1$. So if two $f_{\mu}$ at first and then one $f_{\lambda}$ is applied, the process will be contracted.

Proof of Lemma 2.1.4. First, we prove (2.3) holds when $x=a_{2}$, i.e.

$$
f_{\lambda}\left(\frac{1}{2}-\frac{1}{2 \sqrt{\mu \lambda}}\right)>a_{2} .
$$




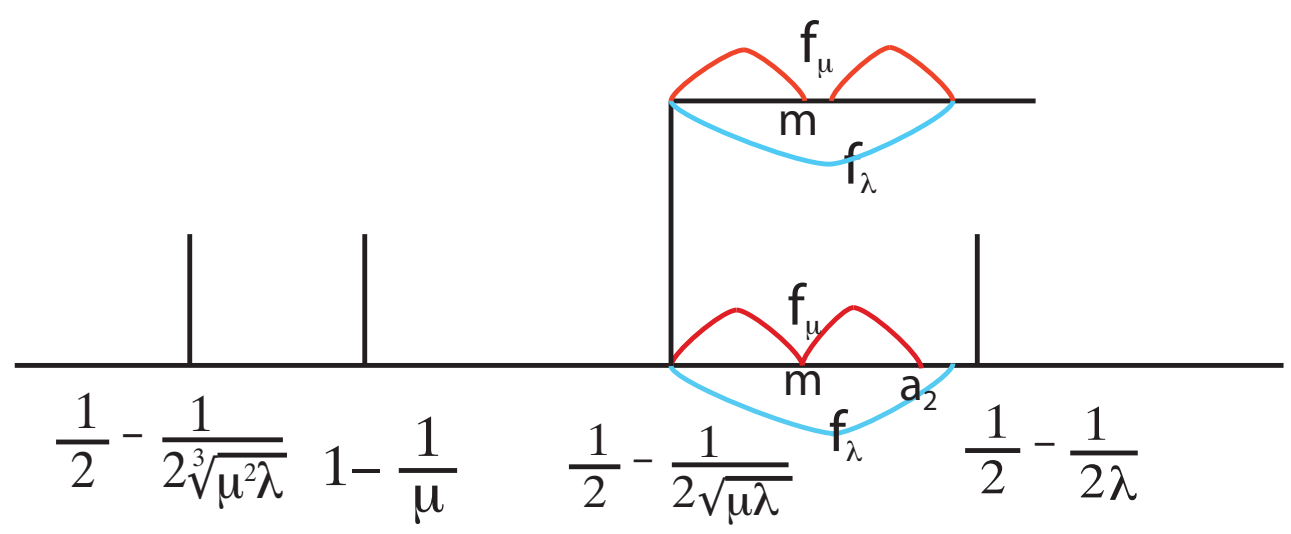

Figure 4.1: $f_{\mu}\left(f_{\lambda}\left(\frac{1}{2}-\frac{1}{2 \sqrt{\mu \lambda}}\right)\right)>m$

For this, we only need to prove the following inequality is true as Figure 4.1 shows

$$
f_{\mu}\left(f_{\lambda}\left(\frac{1}{2}-\frac{1}{2 \sqrt{\mu \lambda}}\right)\right)>m
$$

where $f_{\mu}(m)=\frac{1}{2}-\frac{1}{2 \sqrt{\mu \lambda}}$.

Simple computations give $f_{\mu}\left(f_{\lambda}\left(\frac{1}{2}-\frac{1}{2 \sqrt{\mu \lambda}}\right)\right)=\frac{(\mu \lambda-1)(4 \mu-\mu \lambda+1)}{16 \mu}$ and $m=\frac{\mu-\sqrt{\mu^{2}-2 \mu+2 \sqrt{\frac{\mu}{\lambda}}}}{2 \mu}$, so to prove (4.2) is reduced to prove

$$
A=\frac{(\mu \lambda-1)(4 \mu-\mu \lambda+1)-8 \mu+8 \sqrt{\mu^{2}-2 \mu+2 \sqrt{\frac{\mu}{\lambda}}}}{16 \mu}>0 .
$$

Denote $B(\mu, \lambda)=(\mu \lambda-1)(4 \mu-\mu \lambda+1)-8 \mu+8 \sqrt{\mu^{2}-2 \mu+2 \sqrt{\frac{\mu}{\lambda}}}$ and we differentiate B with respect to $\mu$ to have

$$
\frac{\partial B}{\partial \mu}=8 \mu \lambda-2 \mu \lambda^{2}+2 \lambda-12+4 \frac{2 \mu-2+\frac{1}{\sqrt{\mu \lambda}}}{\sqrt{\mu^{2}-2 \mu+2 \sqrt{\frac{\mu}{\lambda}}}} .
$$

Denote $D=\left(2 \mu-2+\frac{1}{\sqrt{\mu \lambda}}\right)^{2}-\left(\mu^{2}-2 \mu+2 \sqrt{\frac{\mu}{\lambda}}\right)$, then

$$
D=3(\mu-1)^{2}+\frac{\mu \lambda+\sqrt{\mu}(2 \mu-4) \sqrt{\lambda}+1}{\mu \lambda} .
$$

Let $h(\lambda)=\mu \lambda+\sqrt{\mu}(2 \mu-4) \sqrt{\lambda}+1$. It is easy to see that

$$
h^{\prime}(\lambda)=\sqrt{\frac{\mu}{\lambda}}(\sqrt{\mu \lambda}-(2-\mu))>0 .
$$

Then $h(\lambda)$ is an increasing function of $\lambda$ and

$$
h(\lambda)>h(\mu)=3 \mu^{2}-4 \mu+1>0 .
$$


It follows that $D>0$ and then

$$
\frac{\partial B}{\partial \mu}>8 \mu \lambda-2 \mu \lambda^{2}+2 \lambda-8=(8-2 \lambda)(\mu \lambda-1)>0 .
$$

Therefore, $B(\mu, \lambda)$ increases as $\mu$ increases and then $B(\mu, \lambda)>B(1, \lambda)$. But

$$
B(1, \lambda)=-\lambda^{2}+6 \lambda-13+8 \sqrt{\frac{2}{\sqrt{\lambda}}-1} .
$$

Note $\frac{\partial B(1, \lambda)}{\partial \lambda}=\frac{(-2 \lambda+6) \lambda \sqrt{2 \sqrt{\lambda}-\lambda}-4}{\lambda \sqrt{2 \sqrt{\lambda}-\lambda}}$.

Denote $F(\lambda)=(-2 \lambda+6) \lambda \sqrt{2 \sqrt{\lambda}-\lambda}-4$, then

$$
\begin{aligned}
F(\lambda) & =-4 \lambda \sqrt{2 \sqrt{\lambda}-\lambda}-\lambda^{2} \frac{\frac{1}{\sqrt{\lambda}}-1}{\sqrt{2 \sqrt{\lambda}-\lambda}}+6 \sqrt{2 \sqrt{\lambda}-\lambda}+3 \lambda \frac{\frac{1}{\sqrt{\lambda}}-1}{\sqrt{2 \sqrt{\lambda}-\lambda}} \\
& =\frac{5 \lambda^{2}-9 \lambda \sqrt{\lambda}+15 \sqrt{\lambda}-9 \lambda}{\sqrt{2 \sqrt{\lambda}-\lambda}} .
\end{aligned}
$$

Let $E(\lambda)=5 \lambda^{2}-9 \lambda \sqrt{\lambda}+15 \sqrt{\lambda}-9 \lambda$. Then it is easy to see that

$$
E_{\lambda}^{\prime}=10 \lambda-\frac{27}{2} \sqrt{\lambda}-\frac{3}{2}+\frac{15}{2}\left(\frac{1}{\sqrt{\lambda}}-1\right) .
$$

Obviously, $10 \lambda-\frac{27}{2} \sqrt{\lambda}-\frac{3}{2}$ and $\frac{1}{\sqrt{\lambda}}-1$ are both less than 0 when $1<\lambda \leqslant 2$, so $E_{\lambda}^{\prime}<0$ and then $E$ is a decreasing function in $\lambda$. Since $E(1)=2>0$ and $E(2)=2-3 \sqrt{2}<0$, we can find a point a which satisfies $E(a)=0$. As a result, we can have

$$
\begin{cases}F^{\prime}(\lambda)>0 & \text { when } \lambda \in(1, a) \\ F^{\prime}(\lambda)<0 & \text { when } \lambda \in[a, 2]\end{cases}
$$

That means $F(\lambda)$ increases when $\lambda \in(1, a)$ and decreases when $\lambda \in[a, 2]$. Since $F(1)=0$ and $F(2)=4(\sqrt{2 \sqrt{2}-2}-1)<0$, we can find $b>a$ such that $F(b)=0$. This implies

$$
\begin{cases}\frac{\partial B(1, \lambda)}{\partial \lambda}>0, & \text { when } \lambda \in(1, b), \\ \frac{\partial B(1, \lambda)}{\partial \lambda}<0, & \text { when } \lambda \in[b, 2] .\end{cases}
$$

This means $B(1, \lambda)$ is an increasing function of $\lambda$ when $\lambda \in(1, b)$ and a decreasing function when $\lambda \in[b, 2]$. Because $B(1,1)=0$ and $B(1,2)=8 \sqrt{\sqrt{2}-1}-5>0$, so $B(1, \lambda)>$ $0, \quad \forall \lambda \in(1,2]$. Since we have proved that $\mathrm{B}$ is an increasing function with respect to $\mu, B(\mu, \lambda)>B(1, \lambda)>0$ for any $1<\mu<\lambda \leqslant 2$. Therefore, we can get that $A>0$ and (4.1) holds.

To sum up all above, if $f_{\mu}\left(f_{\mu}\left(a_{2}\right)\right)=\frac{1}{2}-\frac{1}{2 \sqrt{\mu \lambda}}, f_{\lambda}\left(\frac{1}{2}-\frac{1}{2 \sqrt{\mu \lambda}}\right)>a_{2}$ holds.

Second, consider $1-\frac{1}{\mu}<x \leqslant a_{2}$. Let $G(x)=f_{\lambda}\left(f_{\mu}\left(f_{\mu}(x)\right)\right)-x$. Note $f_{\lambda}^{\prime \prime}(x)=-2 \lambda$, $f_{\mu}^{\prime \prime}(x)=-2 \mu, f_{\mu}^{\prime}(x)>0$ and $f_{\mu}^{\prime}(x)>0$ for $x \in\left[1-\frac{1}{\mu}, 1-\frac{1}{\lambda}\right]$, then we differentiate $G(x)$ with respect to $x$ to have

$$
G^{\prime}(x)=f_{\lambda}^{\prime}\left(f_{\mu}\left(f_{\mu}(x)\right)\right) \cdot f_{\mu}^{\prime}\left(f_{\mu}(x)\right) \cdot f_{\mu}^{\prime}(x)-1,
$$




$$
\begin{aligned}
G^{\prime \prime}(x)< & f_{\lambda}^{\prime \prime}\left(f_{\mu}\left(f_{\mu}(x)\right)\right) \cdot\left(f_{\mu}^{\prime}\left(f_{\mu}(x)\right) \cdot f_{\mu}^{\prime}(x)\right)^{2}+f_{\lambda}^{\prime}\left(f_{\mu}\left(f_{\mu}(x)\right)\right) \cdot f_{\mu}^{\prime \prime}\left(f_{\mu}(x)\right) \cdot\left(f_{\mu}^{\prime}(x)\right)^{2} \\
& +f_{\lambda}^{\prime}\left(f_{\mu}\left(f_{\mu}(x)\right)\right) \cdot f_{\mu}^{\prime}\left(f_{\mu}(x)\right) \cdot f_{\mu}^{\prime \prime}(x)<0 .
\end{aligned}
$$

Therefore $G(x)$ reaches the minimum valve at $x=1-\frac{1}{\mu}$ or $x=a_{2}$. Since $G\left(1-\frac{1}{\mu}\right)>1-\frac{1}{\mu}$ and $G\left(a_{2}\right)>a_{2}$, then it follows that $(2.3)$ holds for any $x \in\left[1-\frac{1}{\mu}, a_{2}\right]$.

\subsection{3 $\frac{\mu}{(2-\mu)^{3}} \leqslant \lambda \leqslant 2$}

Proof of Lemma 2.1.5. Notice $\lambda \geqslant \frac{\mu}{(2-\mu)^{k}} \geqslant \frac{\mu}{(2-\mu)^{3}}>\frac{\mu}{(2-\mu)^{2}}$. So for any $x \in\left[1-\frac{1}{\mu}, a_{2}\right]$, where $a_{2}$ is defined in Lemma 2.1.4, it follows from Lemma 2.1.4 that

$$
f_{\lambda}\left(f_{\mu}\left(f_{\mu}(x)\right)\right)>x .
$$

Now we use induction method to prove the lemma. First we assume that for a $3 \leqslant k \leqslant n$ and $\lambda \geqslant \frac{\mu}{(2-\mu)^{k-1}}$,

$$
f_{\lambda} \underbrace{f_{\mu} \cdots f_{\mu}}_{k-1}(x)>x, \quad x \in\left[1-\frac{1}{\mu}, a_{k-1}\right],
$$

where $a_{k-1}$ is defined by $\underbrace{f_{\mu} \cdots f_{\mu}}_{k-1}\left(a_{k-1}\right)=\frac{1}{2}-\frac{1}{2 \sqrt[k-1]{\mu^{k-2} \lambda}}$.

Now, assume $\lambda \geqslant \frac{\mu}{(2-\mu)^{k}}$. First, we prove that

$$
f_{\lambda} \underbrace{f_{\mu} \cdots f_{\mu}}_{k}\left(a_{k}\right)>a_{k}
$$

where $a_{k}$ is defined by $\underbrace{f_{\mu} \cdots f_{\mu}}_{k}\left(a_{k}\right)=\frac{1}{2}-\frac{1}{2 \sqrt[k]{\mu^{k-1} \lambda}}$.

For this, we define some $\lambda_{0}$ such that

$$
\left.f_{\mu}\left(f_{\lambda}\left(\frac{1}{2}-\frac{1}{2 \sqrt[k]{\mu^{k-1} \lambda}}\right)\right)=f_{\lambda_{0}}\left(\frac{1}{2}-\frac{1}{2 \sqrt[k]{\mu^{k-1} \lambda}}\right)\right) .
$$

Obviously, $\lambda_{0}<\lambda$. Moreover, computing both sides of (4.5) and solving equation (4.5), we can get

$$
\lambda_{0}=\lambda \cdot\left(\mu-\frac{\mu \lambda}{4}+\frac{1}{4}\left(\frac{\lambda}{\mu}\right)^{\frac{k-2}{k}}\right) .
$$

(i) We prove

$$
\frac{1}{2}-\frac{1}{2 \sqrt[k]{\mu^{k-1} \lambda}}<\frac{1}{2}-\frac{1}{2 \sqrt[k-1]{\mu^{k-2} \lambda_{0}}}
$$

It is equivalent to prove

$$
A=\left(\mu^{k-1} \lambda\right)^{\frac{1}{k}}-\left(\mu^{k-2} \lambda_{0}\right)^{\frac{1}{k-1}}=\mu^{k^{2}-2 k}\left(\mu \lambda^{k-1}-\lambda_{0}^{k}\right)<0 .
$$

Since $\lambda_{0}=\lambda \cdot\left(\mu-\frac{\mu \lambda}{4}+\frac{1}{4}\left(\frac{\lambda}{\mu}\right)^{\frac{k-2}{k}}\right)$, then we can get

$$
\mu \lambda^{k-1}-\lambda_{0}^{k}=\lambda^{k} \cdot\left[\frac{\mu}{\lambda}-\left(\mu-\frac{\mu \lambda}{4}+\frac{1}{4}\left(\frac{\lambda}{\mu}\right)^{\frac{k-2}{k}}\right)^{k}\right] .
$$




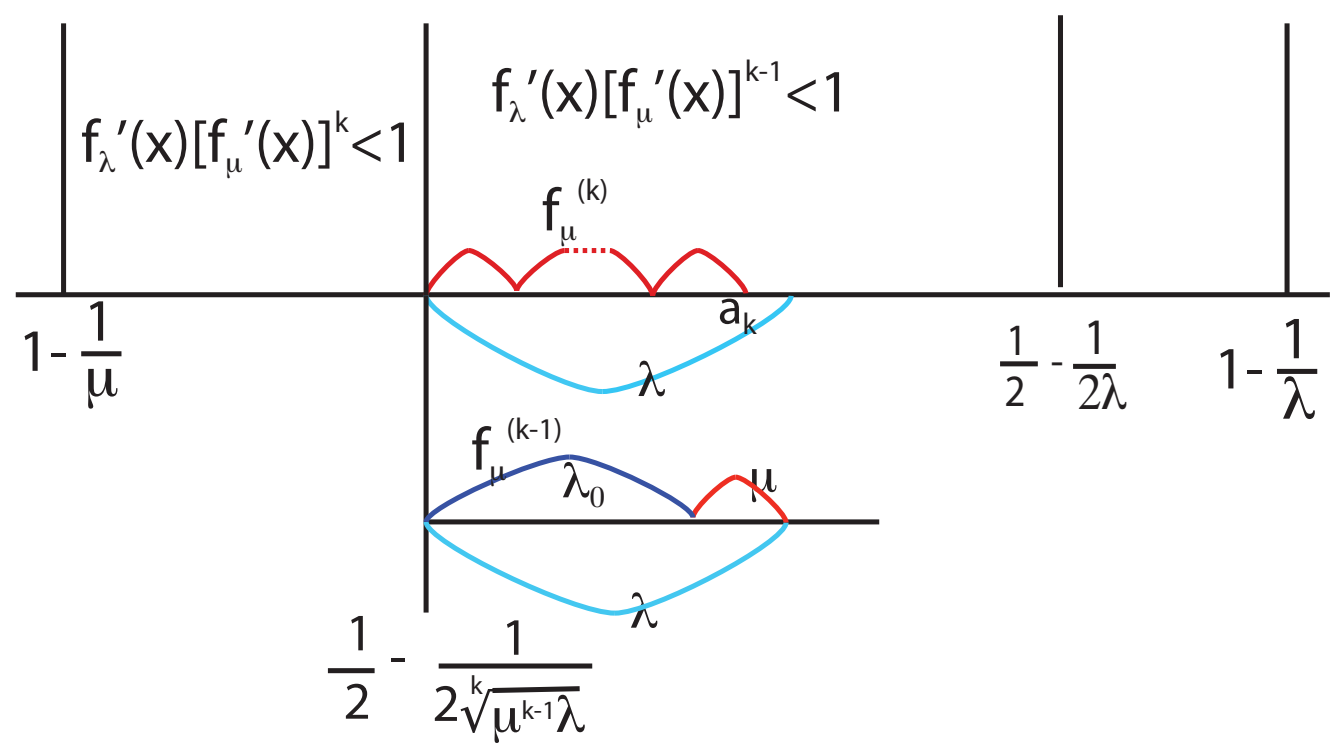

Figure 4.2: $\left.f_{\mu}\left(f_{\lambda}\left(\frac{1}{2}-\frac{1}{2 \sqrt[k]{\mu^{k-1} \lambda}}\right)\right)=f_{\lambda_{0}}\left(\frac{1}{2}-\frac{1}{2 \sqrt[k]{\mu^{k-1} \lambda}}\right)\right)$

Let $B(k)=\mu-\frac{\mu \lambda}{4}+\frac{1}{4}\left(\frac{\lambda}{\mu}\right)^{\frac{k-2}{k}}-\left(\frac{\mu}{\lambda}\right)^{\frac{1}{k}}$. We differentiate $B(k)$ with respect to $k$ to derive that

$$
\begin{aligned}
B^{\prime}(k) & =-\frac{1}{4}\left(\frac{\mu}{\lambda}\right)^{\frac{2-k}{k}} \ln \left(\frac{\mu}{\lambda}\right) \frac{2}{k^{2}}+\left(\frac{\mu}{\lambda}\right)^{\frac{1}{k}} \ln \left(\frac{\mu}{\lambda}\right) \frac{1}{k^{2}} \\
& =\frac{1}{k^{2}}\left(\frac{\mu}{\lambda}\right)^{\frac{1}{k}} \ln \left(\frac{\mu}{\lambda}\right)\left(1-\frac{1}{2}\left(\frac{\mu}{\lambda}\right)^{\frac{1-k}{k}}\right)<0,
\end{aligned}
$$

since $1-\frac{1}{2}\left(\frac{\mu}{\lambda}\right)^{\frac{1-k}{k}}>0$ and $\ln \frac{\mu}{\lambda}<0$. Thus $B$ decreases as $k$ increases. It turns out that for any $k$

$$
B(k)>\lim _{k \rightarrow \infty} B(k)=\mu-\frac{\mu \lambda}{4}+\frac{\lambda}{4 \mu}-1 \geqslant \frac{(\mu-1)^{2}}{2 \mu}>0 .
$$

As a result, $A<0$ and (4.7) holds.

(ii) We prove that

$$
\lambda_{0}>\frac{\mu}{(2-\mu)^{k-1}} .
$$

Differentiating $\lambda_{0}(\lambda)$ with respect to $\lambda$, we can get

$$
\frac{\partial \lambda_{0}(\lambda)}{\partial \lambda}=\mu-\frac{\mu \lambda}{2}+\frac{1}{4}\left(\frac{\lambda}{\mu}\right)^{\frac{k-2}{k}}\left(1+\frac{k-2}{k}\right)>\mu-\frac{\mu \lambda}{2}>0 .
$$

Therefore, $\lambda_{0}(\lambda)$ is an increasing function in $\lambda$ and

$$
\lambda_{0}(\lambda)>\lambda_{0}\left(\frac{\mu}{(2-\mu)^{k}}\right)=\frac{\mu}{(2-\mu)^{k}} \cdot\left[\mu-\frac{\mu^{2}}{4(2-\mu)^{k}}+\frac{(2-\mu)^{2-k}}{4}\right] .
$$


Now, we prove that $\mu-\frac{\mu^{2}}{4(2-\mu)^{k}}+\frac{(2-\mu)^{2-k}}{4}>2-\mu$. Note

$$
D=2 \mu-2-\frac{\mu^{2}}{4(2-\mu)^{k}}+\frac{(2-\mu)^{2-k}}{4}=\frac{1}{4(2-\mu)^{k}} \cdot\left[4(\mu-1)\left(2(2-\mu)^{k}-1\right)\right] .
$$

Notice $\frac{\mu}{(2-\mu)^{k}} \leqslant 2$, so $(2-\mu)^{k} \geqslant \frac{\mu}{2}>\frac{1}{2}$. It follows that $D>0$. Then (4.8) follows easily.

Now it follows from (4.5), (4.7), (4.8), and (4.3) that

$$
\underbrace{f_{\mu} \cdots f_{\mu}}_{k}\left(f_{\lambda}\left(\frac{1}{2}-\frac{1}{2 \sqrt[k]{\mu^{k-1} \lambda}}\right)\right)>\underbrace{f_{\mu} \cdots f_{\mu}}_{k-1}\left(f_{\lambda_{0}}\left(\frac{1}{2}-\frac{1}{2 \sqrt[k]{\mu^{k-1} \lambda}}\right)\right)>\frac{1}{2}-\frac{1}{2 \sqrt[k]{\mu^{k-1} \lambda}} .
$$

Thus, (4.4) holds.

Now considering arbitrary $x \in\left[1-\frac{1}{\mu}, a_{k}\right]$. Note $f_{\lambda}^{\prime \prime}(x)<0, f_{\mu}^{\prime \prime}(x)<0, f_{\mu}^{\prime}(x)>0$,

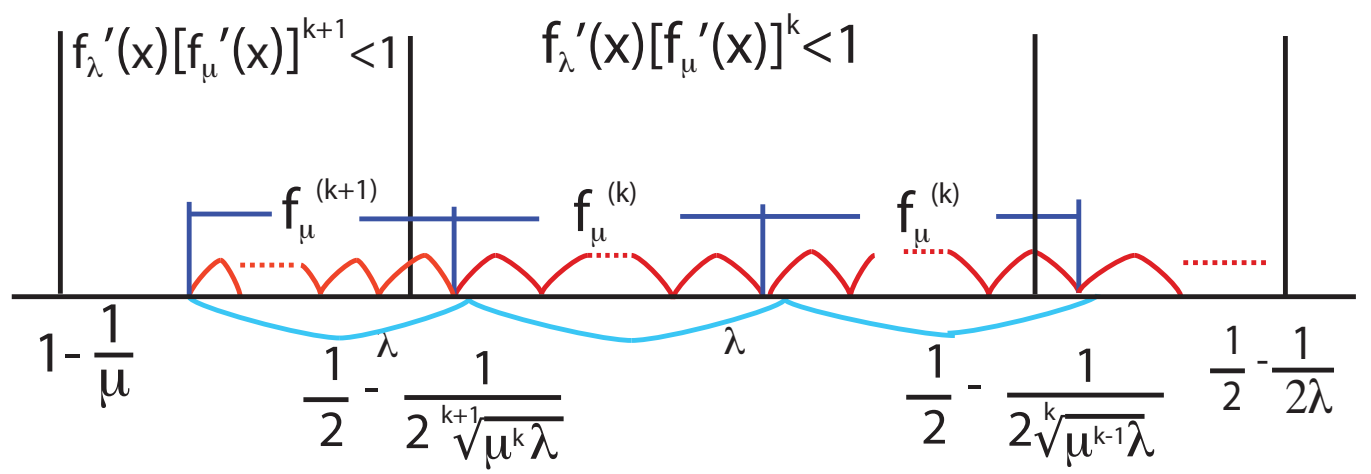

Figure 4.3: Contracted process

$f_{\lambda}^{\prime}(x)>0$ and we have

$$
\begin{aligned}
& \frac{d}{d x}\left[f_{\lambda}(\underbrace{f_{\mu} \cdots f_{\mu}}_{k}(x))-x\right]=f_{\lambda}^{\prime}(\underbrace{f_{\mu} \cdots f_{\mu}}_{k}(x)) \cdot f_{\mu}^{\prime}(\underbrace{f_{\mu} \cdots f_{\mu}}_{k-1}(x)) \cdots f_{\mu}^{\prime}(x)-1, \\
& \frac{d^{2}}{d x^{2}}\left[f_{\lambda}(\underbrace{f_{\mu} \cdots f_{\mu}}_{k}(x))-x\right]=f_{\lambda}^{\prime \prime}(\underbrace{f_{\mu} \cdots f_{\mu}}_{k}(x)) \cdot\left(f_{\mu}^{\prime}(\underbrace{f_{\mu} \cdots f_{\mu}}_{k-1}(x)) \cdots f_{\mu}^{\prime}(x)\right)^{2} \\
& +f_{\lambda}^{\prime}(\underbrace{f_{\mu} \cdots f_{\mu}}_{k}(x)) \cdot f_{\mu}^{\prime \prime}(\underbrace{f_{\mu} \cdots f_{\mu}}_{k-1}(x)) \cdot\left(f_{\mu}^{\prime}(\underbrace{f_{\mu} \cdots f_{\mu}}_{k-2}(x)) \cdots f_{\mu}^{\prime}(x)\right)^{2} \\
& +\cdots+f_{\mu}^{\prime \prime}(x) \cdot f_{\lambda}^{\prime}(\underbrace{f_{\mu} \cdots f_{\mu}}_{k}(x)) \cdot f_{\mu}^{\prime}(\underbrace{f_{\mu} \cdots f_{\mu}}_{k-1}(x)) \cdots f_{\mu}^{\prime}\left(f_{\mu}(x)\right) \\
& <0 \text {. }
\end{aligned}
$$


Therefore $\left(f_{\lambda}(\underbrace{f_{\mu} \cdots f_{\mu}}_{k}(x))-x\right)$ obtains the minimum value at $x=1-\frac{1}{\mu}$ or $x=a_{k}$. Since it is easy to see that $f_{\lambda}(\underbrace{f_{\mu} \cdots f_{\mu}}_{k}\left(1-\frac{1}{\mu}\right))>1-\frac{1}{\mu}$ and we have proved that $f_{\lambda}(\underbrace{f_{\mu} \cdots f_{\mu}}_{k}\left(a_{k}\right))>$ $a_{k}$, it turns out that for any $x \in\left[1-\frac{1}{\mu}, a_{k}\right],(2.4)$ holds.

\section{$4.32<\mu<\lambda \leqslant 3$}

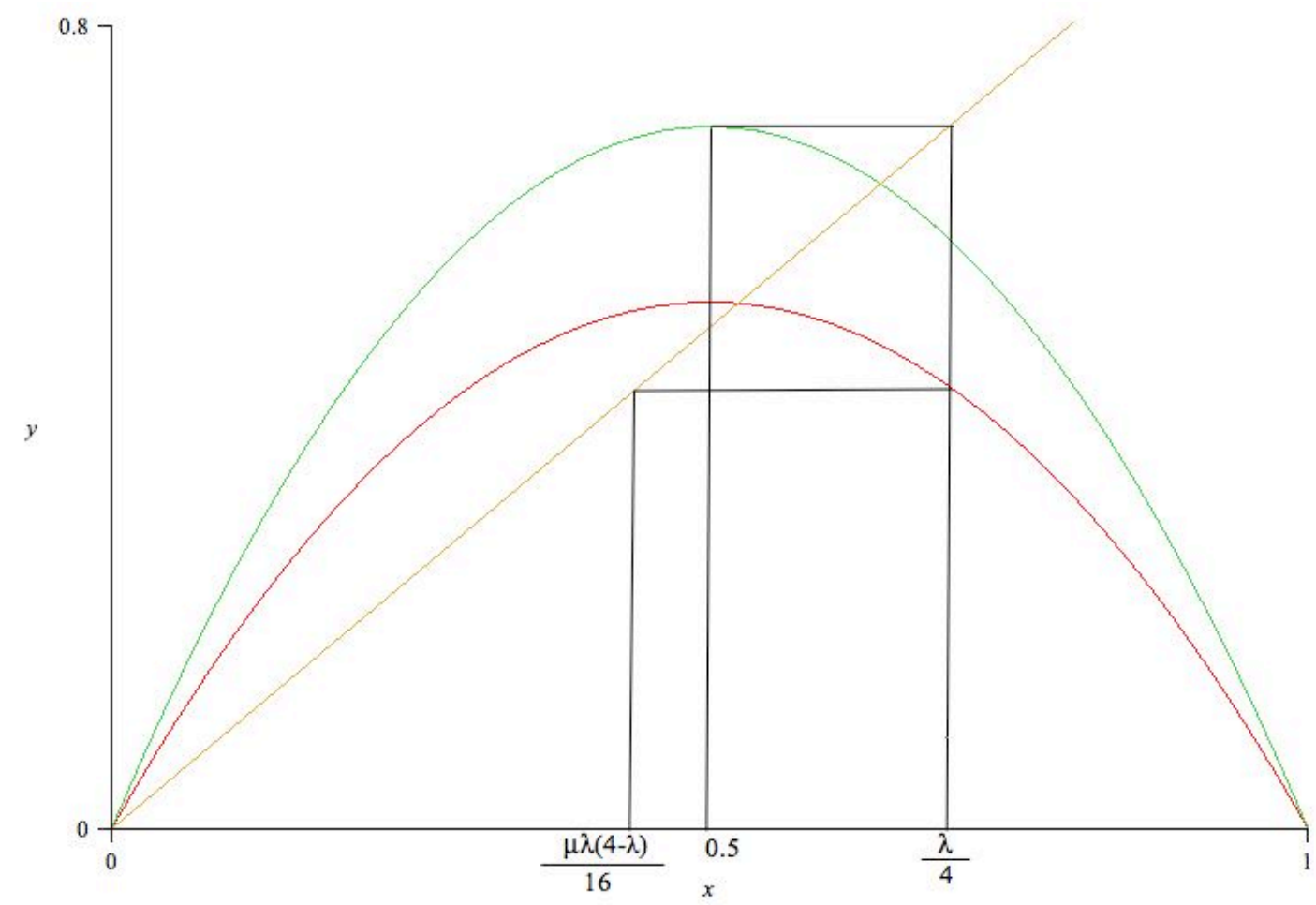

Figure 4.4: $2<\mu<\lambda \leqslant 3$

For any $2<\mu<\lambda \leqslant 3$, it is easy to see that $\left[f_{\mu}\left(\frac{\lambda}{4}\right), \frac{\lambda}{4}\right]$ is an invariant interval. This means that once $x$ enters the interval $\left[\frac{\mu \lambda(4-\lambda)}{16}, \frac{\lambda}{4}\right]$, it will remain there forever under the random action of $f_{\mu}$ and $f_{\lambda}$.

\subsection{1 $2<\lambda<1+\sqrt{3}$}

Considering that

$$
\frac{\mu \lambda(4-\lambda)}{16}>\frac{\lambda(4-\lambda)}{8}>\frac{1}{2}-\frac{1}{2 \lambda}>\frac{1}{2}-\frac{1}{2 \mu}
$$

we can easily get $\left|f_{\epsilon}^{\prime}(x)\right|<1$ for $x \in\left[\frac{\mu \lambda(4-\lambda)}{16}, \frac{1}{2}\right]$ and $\epsilon=\mu, \lambda \in(2,3]$. As Figure 2.7 shows, note when $2<\lambda<1+\sqrt{3}$, we can see that $\frac{\lambda}{4}<\frac{1}{2}+\frac{1}{2 \lambda}$. So $\left|f_{\mu}^{\prime}(x)\right|<1$ and $\left|f_{\lambda}^{\prime}(x)\right|<1$ are satisfied for any $x \in\left[\frac{1}{2}, \frac{\lambda}{4}\right] \subset\left[\frac{1}{2}, \frac{1}{2}+\frac{1}{2 \lambda}\right]$. So it is easy to see that the limit of $f_{\epsilon_{-1}} f_{\epsilon_{-2}} \cdots f_{\epsilon_{-k}}(x)$ exists as $k \rightarrow \infty$ for $x \in\left[\frac{\mu \lambda(4-\lambda)}{16}, \frac{\lambda}{4}\right]$. 
4.3.2 $1+\sqrt{3} \leqslant \lambda \leqslant 3$

Proof of Lemma 2.1.7. From Figure 2.8, we can see that $\left|f_{\lambda}^{\prime}(x)\right| \geqslant 1$ for $x \in\left[\frac{1}{2}+\frac{1}{2 \lambda}, \frac{\lambda}{4}\right]$. So we need to check the sign of $\left|f_{\mu}^{\prime}(x) f_{\lambda}^{\prime}\left(f_{\mu}(x)\right)\right|-1,\left|f_{\mu}^{\prime}(x) f_{\mu}^{\prime}\left(f_{\mu}(x)\right)\right|-1,\left|f_{\lambda}^{\prime}(x) f_{\lambda}^{\prime}\left(f_{\lambda}(x)\right)\right|-$ 1 and $\left|f_{\lambda}^{\prime}(x) f_{\mu}^{\prime}\left(f_{\lambda}(x)\right)\right|-1$ for $x \in\left[\frac{\mu \lambda(4-\lambda)}{16}, \frac{\lambda}{4}\right]$.

Recall the following facts for any $x \in\left[\frac{\mu \lambda(4-\lambda)}{16}, \frac{\lambda}{4}\right]$ :

$$
\begin{aligned}
* & & \left|f_{\lambda}^{\prime}(x)\right|>\left|f_{\mu}^{\prime}(x)\right|, \\
* & & \left|f_{\lambda}(x)\right|>\left|f_{\mu}(x)\right|, \\
* & & \left|f_{\lambda}^{\prime}\left(f_{\lambda}(x)\right)\right|>\left|f_{\mu}^{\prime}\left(f_{\lambda}(x)\right)\right| .
\end{aligned}
$$

First, we will prove for any $x \in\left[\frac{\mu \lambda(4-\lambda)}{16}, \frac{\lambda}{4}\right]$

$$
\begin{aligned}
& \left|f_{\lambda}^{\prime}\left(f_{\lambda}(x)\right) \cdot f_{\lambda}^{\prime}(x)\right| \leqslant 1, \\
& \left|f_{\mu}^{\prime}\left(f_{\lambda}(x)\right) \cdot f_{\lambda}^{\prime}(x)\right| \leqslant 1 .
\end{aligned}
$$

Note $f_{\lambda}^{\prime}\left(f_{\lambda}(x)\right) \cdot f_{\lambda}^{\prime}(x)=\lambda^{2}(1-2 x)\left(1-2 \lambda x+2 \lambda x^{2}\right)$.

Let $g(x)=(1-2 x)\left(1-2 \lambda x+2 \lambda x^{2}\right)$. Differentiate $g(x)$ with respect to $x$ to get

$$
g^{\prime}(x)=-2\left[1-2 \lambda x+2 \lambda x^{2}+\lambda(1-2 x)^{2}\right] .
$$

Solve $g^{\prime}(x)=0$ to get $x=\frac{1}{2} \pm \frac{\sqrt{3 \lambda^{2}-6 \lambda}}{6 \lambda}$. Obviously, when $x \in\left[\frac{1}{2}-\frac{\sqrt{3 \lambda^{2}-6 \lambda}}{6 \lambda}, \frac{1}{2}+\frac{\sqrt{3 \lambda^{2}-6 \lambda}}{6 \lambda}\right)$, $g^{\prime}(x)>0$. When $x \in\left[\frac{1}{2}+\frac{\sqrt{3 \lambda^{2}-6 \lambda}}{6 \lambda}, \frac{\lambda}{4}\right], g^{\prime}(x) \leqslant 0$. It is easy to see that $|g(x)|$ takes maximum valve at $x=\frac{1}{2} \pm \frac{\sqrt{3 \lambda^{2}-6 \lambda}}{6 \lambda}, x=\frac{\mu \lambda(4-\lambda)}{16}$ or $x=\frac{\lambda}{4}$. Moreover, if we Solve $|g(x)|=0$, we can get $x=\frac{1}{2}$ and $x=\frac{1}{2} \pm \frac{\sqrt{\lambda^{2}-2 \lambda}}{2 \lambda}$.

Since $2 \leqslant \lambda^{2}-2 \lambda \leqslant 3$ for $1+\sqrt{3} \leqslant \lambda \leqslant 3$, so we can have $\sqrt{6} \leqslant \sqrt{3 \lambda(\lambda-2)} \leqslant 3$ and

$$
\begin{aligned}
& \left|f_{\lambda}^{\prime}\left(f_{\lambda}\left(\frac{1}{2}+\frac{\sqrt{3 \lambda^{2}-6 \lambda}}{6 \lambda}\right)\right) \cdot f_{\lambda}^{\prime}\left(\frac{1}{2}+\frac{\sqrt{3 \lambda^{2}-6 \lambda}}{6 \lambda}\right)\right| \\
& =\lambda^{2}\left|g\left(\frac{1}{2}+\frac{\sqrt{3 \lambda^{2}-6 \lambda}}{6 \lambda}\right)\right|=\frac{\sqrt{3}\left(\lambda^{2}-2 \lambda\right)^{\frac{3}{2}}}{9} \leqslant 1 .
\end{aligned}
$$

Since $\frac{\mu \lambda(4-\lambda)}{16}>\frac{\lambda(4-\lambda)}{8}$ and $\frac{\sqrt{3 \lambda^{2}-6 \lambda}}{6 \lambda}-\left(\frac{1}{2}-\frac{\lambda(4-\lambda)}{8}\right)=\frac{4-\sqrt{3 \lambda(\lambda-2)^{3}}}{8 \sqrt{3 \lambda}}>0$, i.e. $\frac{\mu \lambda(4-\lambda)}{16}>$ $\frac{1}{2}-\frac{\sqrt{3 \lambda^{2}-6 \lambda}}{6 \lambda}$. So we have

$$
\left|g\left(\frac{\mu \lambda(4-\lambda)}{16}\right)\right|<\left|g\left(\frac{1}{2}-\frac{\sqrt{3 \lambda^{2}-6 \lambda}}{6 \lambda}\right)\right|
$$

It is also easy to see $\frac{\lambda}{4} \in\left(\frac{1}{2}+\frac{\sqrt{3 \lambda^{2}-6 \lambda}}{6 \lambda}, \frac{1}{2}+\frac{\sqrt{\lambda^{2}-2 \lambda}}{2 \lambda}\right)$ from $\frac{1}{2}<\frac{\sqrt{6}}{4} \leqslant \frac{\sqrt{3 \lambda(\lambda-2)}}{4} \leqslant \frac{3}{4}<\frac{\sqrt{3}}{2}$, so

$$
\left|g\left(\frac{\lambda}{4}\right)\right|<g\left(\frac{1}{2}+\frac{\sqrt{3 \lambda^{2}-6 \lambda}}{6 \lambda}\right) .
$$

As a result,

$$
\left|f_{\lambda}^{\prime}\left(f_{\lambda}(x)\right) f_{\lambda}^{\prime}(x)\right|=\lambda^{2}|g(x)| \leqslant \lambda^{2}\left|g\left(\frac{1}{2} \pm \frac{\sqrt{3 \lambda^{2}-6 \lambda}}{6 \lambda}\right)\right| \leqslant 1
$$


That is to say (4.9) holds for $x \in\left[\frac{\mu \lambda(4-\lambda)}{16}, \frac{\lambda}{4}\right]$ and only when $x=\frac{2}{3}$ and $\lambda=3, f_{\lambda}^{\prime}\left(f_{\lambda}(x)\right)$. $f_{\lambda}^{\prime}(x)=1$. Moreover, (4.10) follows easily as

$$
\left|f_{\mu}^{\prime}\left(f_{\lambda}(x)\right) \cdot f_{\lambda}^{\prime}(x)\right|<\left|f_{\lambda}^{\prime}\left(f_{\lambda}(x)\right) \cdot f_{\lambda}^{\prime}(x)\right| .
$$

Second, we prove that for any $x \in\left[\frac{\mu \lambda(4-\lambda)}{16}, \frac{\lambda}{4}\right]$,

$$
\begin{aligned}
& \left.\mid f_{\lambda}^{\prime}\left(f_{\mu}(x)\right)\right] f_{\mu}^{\prime}(x) \mid<1, \\
& \left|f_{\mu}^{\prime}\left(f_{\mu}(x)\right) f_{\mu}^{\prime}(x)\right|<1 .
\end{aligned}
$$

We only need to prove (4.13) as $\left|f_{\mu}^{\prime}\left(f_{\mu}(x)\right)\right|<\left|f_{\lambda}^{\prime}\left(f_{\mu}(x)\right)\right|$.

For this, note $f_{\lambda}^{\prime}\left(f_{\mu}(x)\right) f_{\mu}^{\prime}(x)=\mu \lambda(1-2 x)\left(1-2 \mu x+2 \mu x^{2}\right)$. Let $h(x)=(1-2 x)(1-$ $\left.2 \mu x+2 \mu x^{2}\right)$ and solve $h^{\prime}(x)=0$ to get $x=\frac{1}{2} \pm \frac{\sqrt{3 \mu^{2}-6 \mu}}{6 \mu}$. It is easy to see that $|h(x)|$ attains the maximum value at $x=\frac{1}{2} \pm \frac{\sqrt{3 \mu^{2}-6 \mu}}{6 \mu}, x=\frac{\mu \lambda(4-\lambda)}{16}$ or $x=\frac{\lambda}{4}$. It is obvious to see that

$$
\left|\mu \lambda h\left(\frac{1}{2} \pm \frac{\sqrt{3 \mu^{2}-6 \mu}}{6 \mu}\right)\right|=\mu \lambda \frac{\sqrt{3}\left(\mu^{2}-2 \mu\right)^{\frac{3}{2}}}{9 \mu^{2}}<\frac{\sqrt{3}\left(\mu^{2}-2 \mu\right)^{\frac{3}{2}}}{9}<1 .
$$

We also have

$$
\left|\mu \lambda h\left(\frac{\lambda}{4}\right)\right|=\frac{\lambda(\lambda-2)\left|8 \mu-4 \lambda \mu^{2}+\mu^{2} \lambda^{2}\right|}{16} \leqslant \frac{3\left|\mu^{2}(\lambda-2)^{2}-4 \mu(\mu-2)\right|}{16} .
$$

Denote $E(\lambda)=\frac{3\left|\mu^{2}(\lambda-2)^{2}-4 \mu(\mu-2)\right|}{16}$ and it is easy to see that $E(\lambda)$ increases as $\lambda$ increases and $|E(\lambda)|<\max \{|E(\mu)|,|E(3)|\}<1$. So

$$
\left|f_{\lambda}^{\prime}\left(f_{\mu}\left(\frac{\lambda}{4}\right)\right) f_{\mu}^{\prime}\left(\frac{\lambda}{4}\right)\right|<1
$$

Moreover,

$$
\left|\mu \lambda h\left(\frac{\mu \lambda(4-\lambda)}{16}\right)\right|=\frac{\left|8 \mu \lambda-\mu^{2} \lambda^{2}(4-\lambda)\right|\left|128-16 \mu^{2} \lambda(4-\lambda)+\mu^{3} \lambda^{2}(4-\lambda)^{2}\right|}{1024} .
$$

From (4.15), we can see that $\left|8 \mu \lambda-\mu^{2} \lambda^{2}(4-\lambda)\right|=\lambda\left|8 \mu-4 \lambda \mu^{2}+\mu^{2} \lambda^{2}\right|<16$. Let $\lambda(4-\lambda)=m$, then $m \in[3, \mu(4-\mu))$ and

$$
\begin{aligned}
& \left|128-16 \mu^{2} \lambda(4-\lambda)+\mu^{3} \lambda^{2}(4-\lambda)^{2}\right|=\left|\mu^{3}\left(m-\frac{8}{\mu}\right)^{2}-64 \mu+128\right| \\
& \leqslant \max \left\{|128-64 \mu|,\left|9 \mu^{3}-48 \mu+128\right|,\left|128-16 \mu^{3}(4-\mu)+\mu^{5}(4-\mu)^{2}\right|\right\}<64 .
\end{aligned}
$$

So

$$
\left|f_{\lambda}^{\prime}\left(f_{\mu}\left(\frac{\mu \lambda(4-\lambda)}{16}\right)\right) f_{\mu}^{\prime}\left(\frac{\lambda \mu(4-\lambda)}{16}\right)\right|<1 .
$$

As a result, we can get

$$
\left|f_{\lambda}^{\prime}\left(f_{\mu}(x)\right) f_{\mu}^{\prime}(x)\right|=\mu \lambda|h(x)| \leqslant \mu \lambda\left(\max \left\{\left|h\left(\frac{1}{2} \pm \frac{\sqrt{3 \mu^{2}-6 \mu}}{6 \mu}\right)\right|,\left|h\left(\frac{\mu \lambda(4-\lambda)}{16}\right)\right|,\left|h\left(\frac{\lambda}{4}\right)\right|\right\}\right)<1,
$$


i.e. (4.13)holds. In conclusion, we can get

$$
\begin{aligned}
\left|f_{\epsilon_{2}}^{\prime}\left(f_{\epsilon_{1}}(x)\right) f_{\epsilon_{1}}^{\prime}(x)\right| \leqslant & \max \left\{\left|f_{\lambda}^{\prime}\left(f_{\lambda}\left(\frac{1}{2}+\frac{\sqrt{3 \lambda^{2}-6 \lambda}}{6 \lambda}\right)\right) f_{\lambda}^{\prime}\left(\frac{1}{2}+\frac{\sqrt{3 \lambda^{2}-6 \lambda}}{6 \lambda}\right)\right|,\right. \\
& \left|f_{\lambda}^{\prime}\left(f_{\mu}\left(\frac{\mu \lambda(4-\lambda)}{16}\right)\right) f_{\mu}^{\prime}\left(\frac{\mu \lambda(4-\lambda)}{16}\right)\right|, \mid f_{\lambda}^{\prime}\left(f_{\mu}\left(\frac{\lambda}{4}\right)\right) f_{\mu}^{\prime}\left(\frac{\lambda}{4}\right), \\
& \left.\left|f_{\lambda}^{\prime}\left(f_{\mu}\left(\frac{1}{2} \pm \frac{\sqrt{3 \mu^{2}-6 \mu}}{6 \mu}\right)\right) f_{\mu}^{\prime}\left(\frac{1}{2} \pm \frac{\sqrt{3 \mu^{2}-6 \mu}}{6 \mu}\right)\right|\right\} .
\end{aligned}
$$

So for any $x \in\left[\frac{\mu \lambda(4-\lambda)}{16}, \frac{\lambda}{4}\right]\left|f_{\epsilon_{2}}^{\prime}\left(f_{\epsilon_{1}}(x)\right) f_{\epsilon_{1}}^{\prime}(x)\right|<1$ for any $\epsilon_{1}, \epsilon_{2}=\lambda, \mu$ except the special case when $\epsilon_{1}=\epsilon_{2}=\lambda=3$ and $x=\frac{2}{3}$.

\section{$4.41<\mu \leqslant 2<\lambda \leqslant 3$}

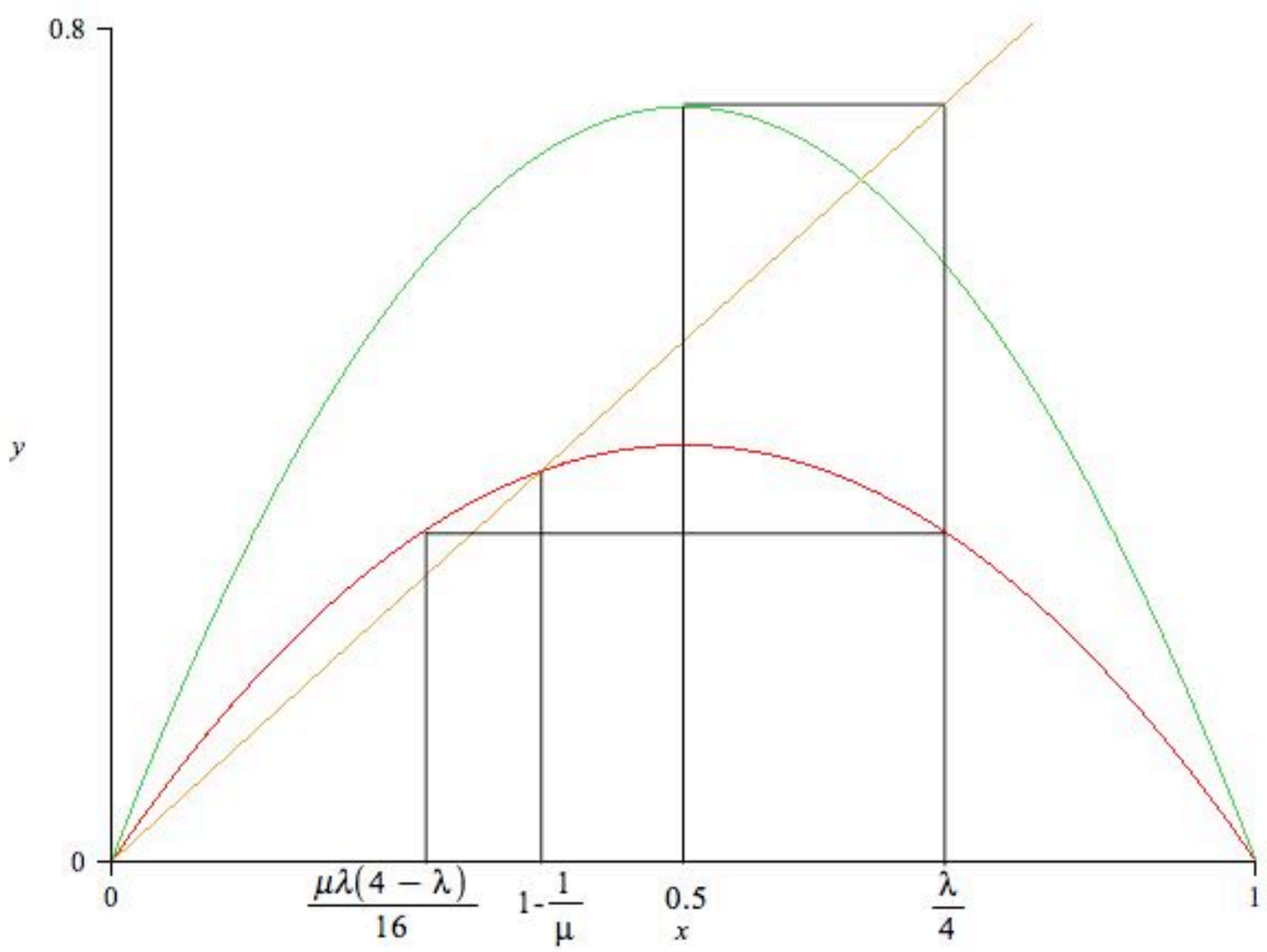

Figure 4.5: $1<\mu \leqslant 2<\lambda \leqslant 3$

We consider

$$
f_{\mu}\left(\frac{\lambda}{4}\right)-\left(\frac{1}{2}-\frac{1}{2 \mu}\right)=\frac{\mu}{16}\left(\lambda(4-\lambda)-\frac{8(\mu-1)}{\mu^{2}}\right) .
$$


Since $\lambda(4-\lambda) \geqslant 3$ for $\lambda \in(2,3]$ and $\frac{8(\mu-1)}{\mu^{2}} \leqslant 2$ for $1<\mu \leqslant 2$, it follows that

$$
f_{\mu}\left(\frac{\lambda}{4}\right)>\left(\frac{1}{2}-\frac{1}{2 \mu}\right)
$$

On the other hand, we consider

$$
f_{\mu}\left(\frac{\lambda}{4}\right)-\left(1-\frac{1}{\mu}\right)=\frac{-\mu^{2}(\lambda-2)^{2}+4(2-\mu)^{2}}{16 \mu}=\frac{[2(2-\mu)+\mu(\lambda-2)](4-\mu \lambda)}{16 \mu} .
$$

Obviously, we can get

$$
\begin{array}{ll}
f_{\mu}\left(\frac{\lambda}{4}\right) \geqslant 1-\frac{1}{\mu}, & \text { if } 1<\mu \leqslant \frac{4}{\lambda}, \\
f_{\mu}\left(\frac{\lambda}{4}\right)<1-\frac{1}{\mu} . & \text { if } \frac{4}{\lambda}<\mu \leqslant 2 .
\end{array}
$$

At first, we will discuss two different cases when $\frac{4}{\lambda}<\mu \leqslant 2$ and when $1<\mu \leqslant \frac{4}{\lambda}$ respectively.

\subsubsection{Case $\frac{4}{\lambda}<\mu \leqslant 2,2<\lambda \leqslant 3$.}

Proof of Lemma 2.1.9. Note $f_{\mu}\left(\frac{\lambda}{4}\right)=\frac{\mu \lambda(4-\lambda)}{16}<1-\frac{1}{\mu}$. Note $\frac{\lambda}{4} \leqslant \frac{1}{2}+\frac{1}{2 \mu}$, so $\left[\frac{\mu \lambda(4-\lambda)}{16}, \frac{\lambda}{4}\right]$ is an invariant interval as Figure 4.5 shows. Then for any combination of $\epsilon_{1}$ and $\epsilon_{2}$ of $\left|f_{\epsilon_{2}}^{\prime}\left(f_{\epsilon_{1}}(x)\right) f_{\epsilon_{1}}^{\prime}(x)\right|$, it is easy to see that $\left|f_{\mu}^{\prime}\left(f_{\mu}(x)\right) f_{\mu}^{\prime}(x)\right|<1$ for any $x \in\left[\frac{\mu \lambda(4-\lambda)}{16}, \frac{\lambda}{4}\right]$ since $\frac{1}{2}-\frac{1}{2 \mu}<\frac{\mu \lambda(4-\lambda)}{16}<\frac{\lambda}{4} \leqslant \frac{1}{2}+\frac{1}{2 \mu}$, Then we need to check the sign of $\left|f_{\lambda}^{\prime}\left(f_{\lambda}(x)\right) f_{\lambda}^{\prime}(x)\right|-1$, $\left|f_{\mu}^{\prime}\left(f_{\lambda}(x)\right) f_{\lambda}^{\prime}(x)\right|-1$ and $\left|f_{\lambda}^{\prime}\left(f_{\mu}(x)\right) f_{\mu}^{\prime}(x)\right|-1$.

(i) We prove $\left|f_{\lambda}^{\prime}\left(f_{\lambda}(x)\right) f_{\lambda}^{\prime}(x)\right|<1 \forall x \in\left[\frac{\mu \lambda(4-\lambda)}{16}, \frac{\lambda}{4}\right]$, recall

$$
f_{\lambda}^{\prime}\left(f_{\lambda}(x)\right) f_{\lambda}^{\prime}(x)=\lambda^{2}(1-2 x)\left(1-2 \lambda x+2 \lambda x^{2}\right):=\lambda^{2} g(x) .
$$

Solve $g^{\prime}(x)=0$ to get $x=\frac{1}{2} \pm \frac{\sqrt{3 \lambda^{2}-6 \lambda}}{6 \lambda}$ and $\frac{\lambda}{4} \in\left[\frac{1}{2}+\frac{\sqrt{3 \lambda^{2}-6 \lambda}}{6 \lambda}, \frac{1}{2}+\frac{\sqrt{\lambda^{2}-2 \lambda}}{2 \lambda}\right]$. It is easy to see that $|g(x)|$ has the maximum value at $x=\frac{1}{2} \pm \frac{\sqrt{3 \lambda^{2}-6 \lambda}}{6 \lambda}$, or $x=\frac{\mu \lambda(4-\lambda)}{16}$. Moreover

$$
\left|\lambda^{2} g\left(\frac{1}{2} \pm \frac{\sqrt{3 \lambda^{2}-6 \lambda}}{6 \lambda}\right)\right|=\lambda^{2}\left|\frac{\sqrt{3}\left(\lambda^{2}-2 \lambda\right)^{\frac{3}{2}}}{9 \lambda^{2}}\right| \leqslant 1
$$

Similar to the case when $2<\mu<\lambda$ and $1+\sqrt{3}<\lambda \leqslant 3$, it is easy to see that $\max \left\{\left|f_{\lambda}^{\prime}\left(f_{\lambda}(x)\right) f_{\lambda}^{\prime}(x)\right|\right\} \leqslant 1$ when $x=\frac{1}{2} \pm \frac{\sqrt{3 \lambda^{2}-6 \lambda}}{6 \lambda}$. On the other hand, we have

$$
\left|\lambda^{2} g\left(\frac{\mu \lambda(4-\lambda)}{16}\right)\right|=\frac{\left|\left(8 \lambda^{2}-\mu \lambda^{3}(4-\lambda)\right)\left(128-16 \mu \lambda^{2}(4-\lambda)+\mu^{2} \lambda^{3}(4-\lambda)^{2}\right)\right|}{1024} .
$$

Let $A(\mu, \lambda)=\left(8 \lambda^{2}-\mu \lambda^{3}(4-\lambda)\right)\left(128-16 \mu \lambda^{2}(4-\lambda)+\mu^{2} \lambda^{3}(4-\lambda)^{2}\right)$, then we differentiate $A(\mu, \lambda)$ with respect to $\mu$ to get

$$
\frac{\partial A(\mu, \lambda)}{\partial \mu}=\lambda^{3}(4-\lambda)\left[-3 \lambda^{3}(4-\lambda)^{2} \mu^{2}+48 \lambda^{2}(4-\lambda) \mu-128(\lambda+1)\right] .
$$


Solving $\frac{\partial A(\mu, \lambda)}{\partial \mu}=0$, we have $\mu=\frac{8}{\lambda(4-\lambda)} \pm \frac{8 \sqrt{3 \lambda^{2}-6 \lambda}}{3 \lambda^{2}(4-\lambda)}$. Since $\frac{8}{\lambda(4-\lambda)}>2$, then for $\mu \in\left(\frac{4}{\lambda}, 2\right],|A(\mu, \lambda)|$ reaches the maximum value at $\mu=\frac{8}{\lambda(4-\lambda)}-\frac{8 \sqrt{3 \lambda^{2}-6 \lambda}}{3 \lambda^{2}(4-\lambda)}$ or $\mu=\frac{4}{\lambda}$ or $\mu=2$. Since $\left(32-8 \lambda^{2}(4-\lambda)+\lambda^{3}(4-\lambda)^{2}\right)^{\prime}=\lambda\left(5 \lambda^{3}-32 \lambda^{2}+72 \lambda-64\right)<0$ for $\lambda \in(2,3]$, then we can get $32-8 \lambda^{2}(4-\lambda)+\lambda^{3}(4-\lambda)^{2} \in[-13,0)$. Moreover,

$$
\begin{aligned}
& A\left(\frac{8}{\lambda(4-\lambda)}-\frac{8 \sqrt{3 \lambda^{2}-6 \lambda}}{3 \lambda^{2}(4-\lambda)}, \lambda\right)=-\frac{64 \cdot 16 \lambda(\lambda-2) \sqrt{3 \lambda^{2}-6 \lambda}}{9} \geqslant-64 \cdot 16=-1024, \\
& A(2, \lambda)=8 \lambda^{2}(\lambda-2)^{2}\left[32-8 \lambda^{2}(4-\lambda)+\lambda^{3}(4-\lambda)^{2}\right] \geqslant 8 \cdot 3^{2} \cdot(-13)>-1024 \\
& A\left(\frac{4}{\lambda}, \lambda\right)=64 \lambda^{2}(\lambda-2)\left(\lambda^{3}-4 \lambda^{2}+8\right)>64 \cdot 3^{2}\left(\left(\frac{8}{3}\right)^{3}-4 \cdot\left(\frac{8}{3}\right)^{2}+8\right)>-1024 .
\end{aligned}
$$

So $\left|f_{\lambda}^{\prime}\left(f_{\lambda}\left(\frac{\mu \lambda(4-\lambda)}{16}\right)\right) f_{\lambda}^{\prime}\left(\frac{\mu \lambda(4-\lambda)}{16}\right)\right| \leqslant 1$. Considering (4.17), we caN get the conclusion that $\left|f_{\lambda}^{\prime}\left(f_{\lambda}(x)\right) f_{\lambda}^{\prime}(x)\right| \leqslant 1 \quad \lambda \in(2,3]$. Note that when $\lambda \in(2,3)$, we have $\left|f_{\lambda}^{\prime}\left(f_{\lambda}(x)\right) f_{\lambda}^{\prime}(x)\right|<1$ and

$$
\begin{aligned}
\left|f_{\lambda}^{\prime}\left(f_{\lambda}(x)\right) f_{\lambda}^{\prime}(x)\right| \leqslant & \max \left\{f_{\lambda}^{\prime}\left(f_{\lambda}\left(\frac{1}{2} \pm \frac{\sqrt{3 \lambda^{2}-6 \lambda}}{6 \lambda}\right)\right) f_{\lambda}^{\prime}\left(\frac{1}{2} \pm \frac{\sqrt{3 \lambda^{2}-6 \lambda}}{6 \lambda}\right)\right. \\
& \left.f_{\lambda}^{\prime}\left(f_{\lambda}\left(\frac{\mu \lambda(4-\lambda)}{16}\right)\right) f_{\lambda}^{\prime}\left(\frac{\mu \lambda(4-\lambda)}{16}\right)\right\}=L_{1}^{\prime} \leqslant 1 .
\end{aligned}
$$

However, when $\lambda=3$, it is possible for $\left|f_{\lambda}^{\prime}\left(f_{\lambda}\left(\frac{1}{3}\right)\right) f_{\lambda}^{\prime}\left(\frac{1}{3}\right)\right|=1$ and $\left|f_{\lambda}^{\prime}\left(f_{\lambda}\left(\frac{2}{3}\right)\right) f_{\lambda}^{\prime}\left(\frac{2}{3}\right)\right|=$ 1, Otherwise, $\left|f_{\lambda}^{\prime}\left(f_{\lambda}(x)\right) f_{\lambda}^{\prime}(x)\right|<1$.

(ii) We prove $\left|f_{\lambda}^{\prime}\left(f_{\mu}(x)\right) f_{\mu}^{\prime}(x)\right|<1$ for $x \in\left[\frac{\mu \lambda(4-\lambda)}{16}, \frac{\lambda}{4}\right]$. Similar to the case when $2<$ $\mu<\lambda \leqslant 3$, we have

$$
\begin{aligned}
f_{\lambda}^{\prime}\left(f_{\mu}(x)\right) f_{\mu}^{\prime}(x) & =\lambda \mu(1-2 x)\left(1-2 \mu x+2 \mu x^{2}\right), \\
\text { Denote } h(x) & =(1-2 x)\left(1-2 \mu x+2 \mu x^{2}\right), \\
\text { then } h^{\prime}(x) & =-12 \mu x^{2}+12 \mu x-2 \mu-2 .
\end{aligned}
$$

Note when $x=\frac{1}{2} \pm \frac{\sqrt{3 \mu^{2}-6 \mu}}{6 \mu}, h^{\prime}(x)=0$ and $\mu \lambda h\left(\frac{1}{2} \pm \frac{\sqrt{3 \mu^{2}-6 \mu}}{6 \mu}\right)<1$. As a result, we only need to check $\left|f_{\lambda}^{\prime}\left(f_{\mu}(x)\right) f_{\mu}^{\prime}(x)\right|<1$ at $x=\frac{\mu \lambda(4-\lambda)}{16}$ and $x=\frac{\lambda}{4}$. Because $\left|\mu\left(8-4 \lambda \mu+\mu \lambda^{2}\right)\right|<\max \{|\mu(8-4 \mu)|,|\mu(8-3 \mu)|\} \leqslant \frac{16}{3}$ and $\lambda-2 \leqslant 1$, we have

$$
\left|f_{\lambda}^{\prime}\left(f_{\mu}\left(\frac{\lambda}{4}\right)\right) f_{\mu}^{\prime}\left(\frac{\lambda}{4}\right)\right|=\frac{\lambda \mu(\lambda-2)\left|8-4 \lambda \mu+\mu \lambda^{2}\right|}{16}<1 .
$$

On the other hand,

$$
\mu \lambda h\left(\frac{\mu \lambda(4-\lambda)}{16}\right)=\frac{\left(8 \mu \lambda-\mu^{2} \lambda^{2}(4-\lambda)\right)\left(128-16 \mu^{2} \lambda(4-\lambda)+\mu^{3} \lambda^{2}(4-\lambda)^{2}\right)}{1024} .
$$

Let $H(\mu)=\left(8 \mu \lambda-\mu^{2} \lambda^{2}(4-\lambda)\right)\left(128-16 \mu^{2} \lambda(4-\lambda)+\mu^{3} \lambda^{2}(4-\lambda)^{2}\right)$ and we differentiate $H$ with respect to $\mu$ to get

$$
\begin{aligned}
H^{\prime}(\mu)= & 2 \lambda(4-\mu \lambda(4-\lambda))\left(128-16 \mu^{2} \lambda(4-\lambda)+\mu^{3} \lambda^{2}(4-\lambda)^{2}\right) \\
& +\mu \lambda(8-\mu \lambda(4-\lambda)) \mu \lambda(4-\lambda)(-32+3 \mu \lambda(4-\lambda)) .
\end{aligned}
$$


Because $4-\mu \lambda(4-\lambda)<4-4(4-\lambda)<0$ and $-32+3 \mu \lambda(4-\lambda)<-32+6 \lambda(4-\lambda)<0$ when $\mu \in\left(\frac{4}{\lambda}, 2\right]$, it is easy to get $H^{\prime}(\mu)<0$ and $H(\mu)$ increases as $\mu$ decreases. So

$$
H(\mu)<H\left(\frac{4}{\lambda}\right)=1024(\lambda-2)^{2} \leqslant 1024 .
$$

By using the same methods in (i), we can show that for any $x \in\left[1-\frac{1}{\mu}, \frac{\lambda}{4}\right]$, $\left|f_{\lambda}^{\prime}\left(f_{\mu}\left(\frac{\mu \lambda(4-\lambda)}{16}\right)\right) f_{\mu}^{\prime}\left(\frac{\lambda \mu(4-\lambda)}{16}\right)\right|<1$.

$$
\begin{aligned}
\left|f_{\lambda}^{\prime}\left(f_{\mu}(x)\right) f_{\mu}^{\prime}(x)\right| \leqslant & \max \left\{\left|f_{\lambda}^{\prime}\left(f_{\mu}\left(\frac{\mu \lambda(4-\lambda)}{16}\right)\right) f_{\mu}^{\prime}\left(\frac{\lambda \mu(4-\lambda)}{16}\right)\right|,\left|f_{\lambda}^{\prime}\left(f_{\mu}\left(\frac{\lambda}{4}\right)\right) f_{\mu}^{\prime}\left(\frac{\lambda}{4}\right)\right|\right. \\
& \left.\left|f_{\lambda}^{\prime}\left(f_{\mu}\left(\frac{1}{2} \pm \frac{\sqrt{3 \mu^{2}-6 \mu}}{6 \mu}\right)\right) f_{\mu}^{\prime}\left(\frac{1}{2} \pm \frac{\sqrt{3 \mu^{2}-6 \mu}}{6 \mu}\right)\right|\right\} \\
= & L_{2}^{\prime}<1 .
\end{aligned}
$$

(iii) We will prove that $\left|f_{\mu}^{\prime}\left(f_{\lambda}(x)\right) f_{\lambda}^{\prime}(x)\right|<1$ for any $x \in\left[\frac{\mu \lambda(4-\lambda)}{16}, \frac{\lambda}{4}\right]$. Note

$$
f_{\mu}^{\prime}\left(f_{\lambda}(x)\right) f_{\lambda}^{\prime}(x)=\mu \lambda(1-2 x)\left(1-2 \lambda x+2 \lambda x^{2}\right)=\mu \lambda g(x) .
$$

Similar as before, it is easy to see that $\left|f_{\mu}^{\prime}\left(f_{\lambda}(x)\right) f_{\lambda}^{\prime}(x)\right|$ reaches the maximum value at $x=\frac{1}{2} \pm \frac{\sqrt{3 \lambda^{2}-6 \lambda}}{6 \lambda}$ or at $x=\frac{\mu \lambda(4-\lambda)}{16}$. Calculations show

$$
\left|\mu \lambda h\left(\frac{1}{2} \pm \frac{\sqrt{3 \lambda^{2}-6 \lambda}}{6 \lambda}\right)\right|=\frac{\mu \sqrt{3}\left(\lambda^{2}-2 \lambda\right)^{\frac{3}{2}}}{9 \lambda}<\frac{\sqrt{3}\left(\lambda^{2}-2 \lambda\right)^{\frac{3}{2}}}{9}<1 .
$$

Moreover,

$$
\left|\mu \lambda h\left(\frac{\mu \lambda(4-\lambda)}{16}\right)\right|=\frac{\left|\left(8 \mu \lambda-\mu^{2} \lambda^{2}(4-\lambda)\right)\left(128-16 \mu \lambda^{2}(4-\lambda)+\mu^{2} \lambda^{3}(4-\lambda)^{2}\right)\right|}{1024} .
$$

Let $A=\left(8 \mu \lambda-\mu^{2} \lambda^{2}(4-\lambda)\right)\left(128-16 \mu \lambda^{2}(4-\lambda)+\mu^{2} \lambda^{3}(4-\lambda)^{2}\right)$ and $B(\mu)=$ $\left.128-16 \mu \lambda^{2}(4-\lambda)+\mu^{2} \lambda^{3}(4-\lambda)^{2}\right)$. We differentiate $B(\mu)$ with respect to $\mu$ to get

$$
B^{\prime}(\mu)=2 \lambda^{2}(4-\lambda)(\mu \lambda(4-\lambda)-8)<0 .
$$

So $B(\mu)$ decreases as $\mu$ increases and $B$ has the maximum value at $\mu=\frac{4}{\lambda}$. Therefore,

$$
\begin{aligned}
& B(\mu)<B\left(\frac{4}{\lambda}\right)=128-64 \lambda(4-\lambda)+16 \lambda(4-\lambda)^{2}=16\left(8-\lambda^{2}(4-\lambda)\right)<0, \\
& \text { and } B(\mu) \geqslant B(2)=4\left(32-8 \lambda^{2}(4-\lambda)+\lambda^{3}(4-\lambda)^{2}\right) .
\end{aligned}
$$

Let $\left.D(\lambda)=-8 \lambda^{2}(4-\lambda)+\lambda^{3}(4-\lambda)^{2}\right)=\lambda^{5}-8 \lambda^{4}+24 \lambda^{3}-32 \lambda^{2}$, then

$$
\begin{array}{ll} 
& D^{\prime}(\lambda)=\lambda\left(5 \lambda^{3}-32 \lambda^{2}+72 \lambda-64\right), \\
\text { and } \quad\left(5 \lambda^{3}-32 \lambda^{2}+72 \lambda-64\right)^{\prime}=15 \lambda^{2}-64 \lambda+72>0 .
\end{array}
$$

So $D^{\prime}(\lambda)<0$. Therefore, we have $D>D(3)=-45$ and $B(2)=4(32+D(\lambda))>-52$. Recalling that $\left(8 \mu \lambda-\mu^{2} \lambda^{2}(4-\lambda)\right)<16$, we can get $|A|<\frac{16 \times 52}{1024}<1$. As a result, for $x \in\left[\frac{\mu \lambda(4-\lambda)}{16}, \frac{\lambda}{4}\right]$, we can get

$$
\begin{aligned}
\left|f_{\mu}^{\prime}\left(f_{\lambda}(x)\right) f_{\lambda}^{\prime}(x)\right| \leqslant & \max \left\{\left|f_{\mu}^{\prime}\left(f_{\lambda}\left(\frac{1}{2} \pm \frac{\sqrt{3 \lambda^{2}-6 \lambda}}{6 \lambda}\right)\right) f_{\lambda}^{\prime}\left(\frac{1}{2} \pm \frac{\sqrt{3 \lambda^{2}-6 \lambda}}{6 \lambda}\right)\right|,\right. \\
& \left.\left|f_{\mu}^{\prime}\left(f_{\lambda}\left(\frac{\mu \lambda(4-\lambda)}{16}\right)\right) f_{\lambda}^{\prime}\left(\frac{\mu \lambda(4-\lambda)}{16}\right)\right|\right\}=L_{3}^{\prime}<1 .
\end{aligned}
$$




\subsubsection{Case $1<\mu<\frac{4}{\lambda}<2<\lambda \leqslant 3$}

In this case, $f_{\mu}\left(\frac{\lambda}{4}\right)>1-\frac{1}{\mu}$, so $\left[\frac{\mu \lambda(4-\lambda)}{16}, \frac{\lambda}{4}\right]$ is no longer an invariant interval. See Figure 4.6 .

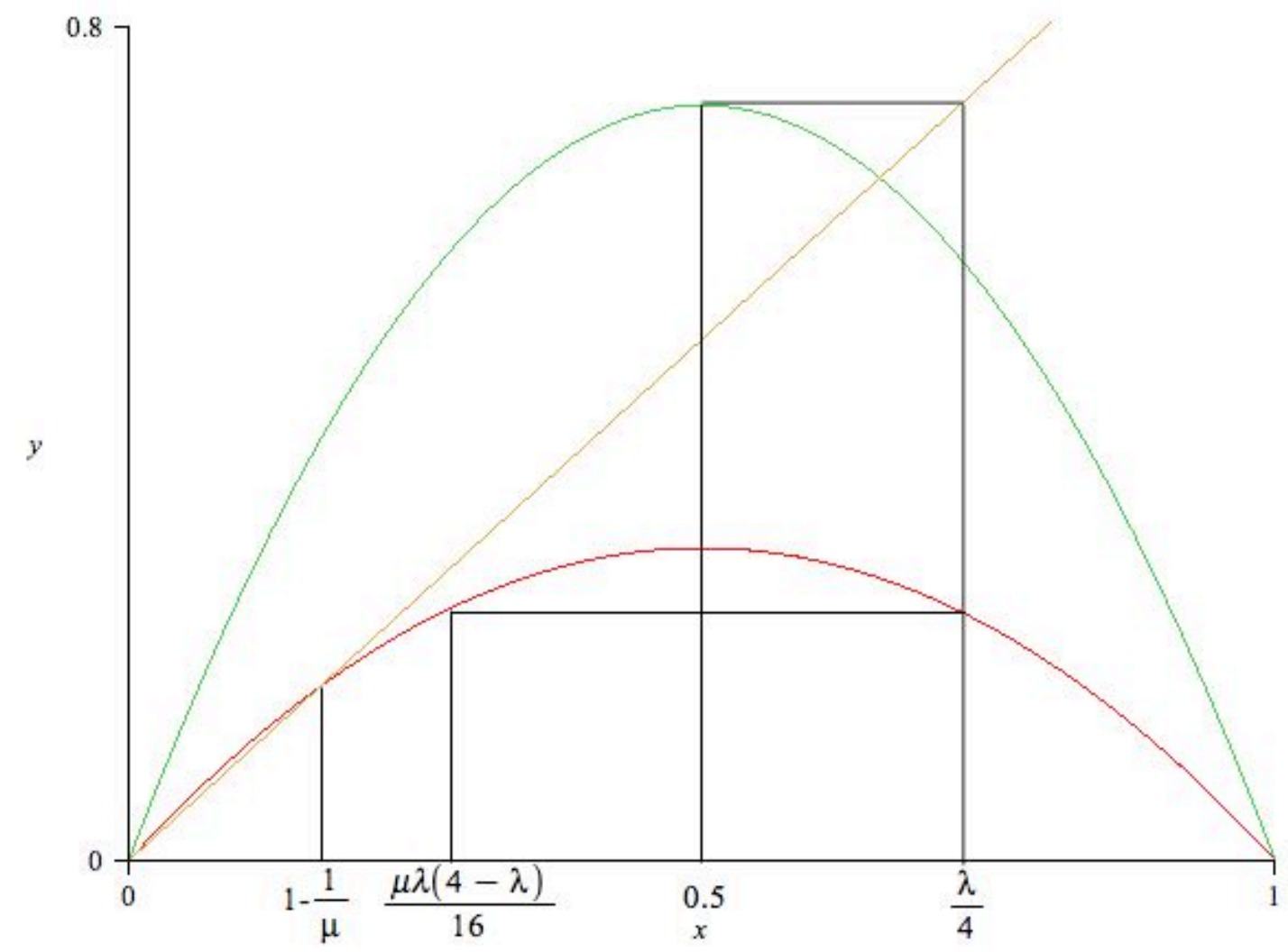

Figure 4.6: $1-\frac{1}{\mu}<\frac{\mu \lambda(4-\lambda)}{16}$

Proof of Lemma 2.1.10. Actually, we just need to prove that Lemma 2.1.3 still holds for $2<\lambda \leqslant 3$. Recalling that in the proof of Lemma 2.1.3, $F(x)=x G(x)$ and $G\left(\frac{1}{2}-\frac{1}{2 \lambda}\right)=$ $\frac{A(\mu, \lambda)}{8 \lambda^{2}}$. Since $A(\mu, \lambda)$ is an increasing function about $\mu$ for $\mu \in(1,2]$ and $A(1, \lambda)=$ $3 \lambda^{3}-5 \lambda^{2}+\lambda+1>0$ for $2<\lambda \leqslant 3$, we can get $F\left(\frac{1}{2}-\frac{1}{2 \lambda}\right)>0$ and Lemma 2.1.10 holds.

Proof of Lemma 2.1.11. First, we will find the condition for the inequality (2.30). Note

$$
\begin{aligned}
& f_{\lambda}\left(f_{\mu}\left(\frac{1}{2}-\frac{1}{2 \sqrt{\mu \lambda}}\right)\right)-\left(\frac{1}{2}-\frac{1}{2 \lambda}\right)=\frac{(\mu \lambda-1)(4 \lambda-\mu \lambda+1)-8 \lambda+8}{16 \lambda}:=\frac{N(\mu)}{16 \lambda} \\
& \frac{\partial N}{\partial \mu}=2 \lambda(2 \lambda-\mu \lambda+1)>0
\end{aligned}
$$

So $N$ increases as $\mu$ increases and $N>N(1)=(3 \lambda-7)(\lambda-1)$. Obviously, when $\lambda \in\left[\frac{7}{3}, 3\right]$, $N>0$. So (2.30) holds. 
However, when $\lambda \in\left(2, \frac{7}{3}\right)$, we will check whether Lemma 2.1 .4 still holds. In the proof of Lemma 2.1.4, $A=f_{\mu}\left(f_{\lambda}\left(\frac{1}{2}-\frac{1}{2 \sqrt{\mu \lambda}}\right)\right)-m=\frac{B(\mu, \lambda)}{16 \mu}$. When $\lambda \in\left(2, \frac{7}{3}\right], h^{\prime}(\lambda)>0, h(\lambda)$ is still an increasing function and $h(\lambda)>0$, then $D>0$ and $\frac{\partial B}{\partial \mu}>8 \mu \lambda-2 \mu \lambda^{2}+2 \lambda+8>0$. So $B$ increases as $\mu$ increases and $B>B(1, \lambda)$. Similar to the proof of Lemma 2.1.4, we also can get $B(1, \lambda)$ increases first and then decrease for $2<\lambda<\frac{7}{3}$. Because $B(1,2)>0$ and $B\left(1, \frac{7}{3}\right)>0$, it is easy to get $B(1, \lambda)>0$ and then Lemma 2.1.4 holds for $2<\lambda<\frac{7}{3}$. So Lemma 2.1.11 is proved.

Proof of Lemma 2.1.12. First, we will prove (2.31) holds when $x=b_{2}$, i.e. $f_{\lambda}\left(f_{\mu}\left(\frac{1}{2}-\right.\right.$ $\left.\left.\frac{1}{2 \sqrt{\mu \lambda}}\right)\right)>b_{2}$. Let $H=f_{\lambda}\left(f_{\mu}\left(\frac{1}{2}-\frac{1}{2 \sqrt{\mu \lambda}}\right)\right)-b_{2}$ and $L(\mu, \lambda)=16 \mu \lambda H$. Then

$$
L(\mu, \lambda)=\mu(\mu \lambda-1)(4 \lambda-\mu \lambda+1)-8 \mu \lambda+8 \lambda \sqrt{\mu^{2}-2 \mu+2 \sqrt{\frac{\mu}{\lambda}}},
$$

and the derivative of $L(\mu, \lambda)$ with respect to $\mu$ is

$$
\frac{\partial L(\mu, \lambda)}{\partial \mu}=(2 \mu \lambda-1)(4 \lambda-\mu \lambda+1)-\lambda\left(\mu^{2} \lambda-\mu\right)-8 \lambda+4 \lambda \frac{2 \mu-2+\frac{1}{\sqrt{\mu \lambda}}}{\sqrt{\mu^{2}-2 \mu+2 \sqrt{\frac{\mu}{\lambda}}}} .
$$

Similar to the proof of Lemma 2.1.4, we can also get $\frac{2 \mu-2+\frac{1}{\sqrt{\mu \lambda}}}{\sqrt{\mu^{2}-2 \mu+2 \sqrt{\frac{\mu}{\lambda}}}}>1$ when $2<\lambda \leqslant 3$. So we have

$$
\frac{\partial L}{\partial \mu}=(2 \mu \lambda-1)(4 \lambda-\mu \lambda+1)-\lambda\left(\mu^{2} \lambda-\mu\right)-4 \lambda=: R(\mu) .
$$

Then

$$
\begin{aligned}
R(\mu) & =-3 \mu^{2} \lambda^{2}+8 \mu \lambda^{2}+4 \mu \lambda-8 \lambda-1 \\
& =-3 \lambda^{2}\left(\mu-\frac{4 \lambda+2}{3 \lambda}\right)^{2}+\frac{16 \lambda^{2}-8 \lambda+1}{3} .
\end{aligned}
$$

Because $\frac{4 \lambda+2}{3 \lambda}>1$, we need to prove $R(1)>0$ and $R\left(\frac{4}{\lambda}\right)>0$ in order to prove $R>0$ for all $1<\mu<\frac{4}{\lambda}$. But this is trivial to see as

$$
\begin{aligned}
& R(1)=(5 \lambda+1)(\lambda-1)>0, \\
& R\left(\frac{4}{\lambda}\right)=24 \lambda-33>0 .
\end{aligned}
$$

Therefore, $\frac{\partial L}{\partial \mu}>0$.So $L(\mu, \lambda)$ is an increasing function with respect to $\mu$. It turns out that

$$
L>L(1, \lambda)=3 \lambda^{2}-10 \lambda-1+8 \lambda \sqrt{-1+\frac{2}{\sqrt{\lambda}}} .
$$

To check the sign of $L(1, \lambda)$, we compute the derivative

$$
\frac{\partial L(1, \lambda)}{\partial \lambda}=6 \lambda-10+\frac{\frac{12}{\sqrt{\lambda}}-8}{\sqrt{-1+\frac{2}{\sqrt{\lambda}}}}
$$


Obviously, when $\frac{12}{\sqrt{\lambda}}-8 \geqslant 0$, so $\frac{\partial L(1, \lambda)}{\partial \lambda}>0$. But when $\lambda \in\left(\frac{9}{4}, 3\right), \frac{12}{\sqrt{\lambda}}-8>-1.072$, $\sqrt{-1+\frac{2}{\sqrt{\lambda}}}>0.39$ and $6 \lambda-10>\frac{7}{2}$, we can get $\frac{\partial L(1, \lambda)}{\partial \lambda}>\frac{7}{2}-\frac{1.072}{0.39}>0$. As a result, $L(1, \lambda)$ increases as $\lambda$ increases and we have

$$
L(\mu, \lambda)>L(1, \lambda)>L(1,2)>0 .
$$

So $H>0$. Therefore (2.31) holds for $x=b_{2}$. Now let $G(x)=f_{\lambda}\left(f_{\mu}\left(f_{\mu}(x)\right)\right)-x$ and because $f_{\eta}^{\prime}(x)=\eta(1-2 x)>0$ for $x \in\left(0, \frac{1}{2}\right)$ and $f_{\eta}^{\prime \prime}(x)=-2 \eta<0$, then we have

$$
\begin{aligned}
G^{\prime}(x)= & f_{\lambda}^{\prime}\left(f_{\mu}\left(f_{\mu}(x)\right)\right) \cdot f_{\mu}^{\prime}\left(f_{\mu}(x)\right) \cdot f_{\mu}^{\prime}(x)-1, \\
G^{\prime \prime}(x)= & f_{\lambda}^{\prime \prime}\left(f_{\mu}\left(f_{\mu}(x)\right)\right) \cdot\left(f_{\mu}^{\prime}\left(f_{\mu}(x)\right) \cdot f_{\mu}^{\prime}(x)\right)^{2}+f_{\mu}^{\prime \prime}\left(f_{\mu}(x)\right) \cdot f_{\lambda}^{\prime}\left(f_{\mu}\left(f_{\mu}(x)\right) \cdot\left(f_{\mu}^{\prime}(x)\right)^{2}\right. \\
& +\left(f_{\lambda}^{\prime}\left(f_{\mu}\left(f_{\mu}(x)\right)\right) \cdot f_{\mu}^{\prime}\left(f_{\mu}(x)\right)\right) \cdot f_{\mu}^{\prime \prime}(x) \\
= & -2 \lambda\left(f_{\mu}^{\prime}\left(f_{\mu}(x)\right) \cdot f_{\mu}^{\prime}(x)\right)^{2}-2 \mu f_{\lambda}^{\prime}\left(f_{\mu}\left(f_{\mu}(x)\right) \cdot\left(f_{\mu}^{\prime}(x)\right)^{2}\right. \\
& -2 \mu f_{\lambda}^{\prime}\left(f_{\mu}\left(f_{\mu}(x)\right)\right) \cdot f_{\mu}^{\prime}\left(f_{\mu}(x)\right)<0 .
\end{aligned}
$$

Therefore, $G(x)$ increases first and then decreases as $x$ increase. Considering that $G(1-$ $\left.\frac{1}{\mu}\right)>0$ and $G\left(b_{2}\right)>0$, we can get $(2.31)$ holds for $x \in\left[1-\frac{1}{\mu}, b_{2}\right]$.

Proof of Lemma 2.1.14. Let $f_{\mu}(m)=\frac{1}{2}-\frac{1}{2 \sqrt[k]{\mu^{k-1} \lambda}}$, then $m=\frac{1}{2}-\frac{\sqrt{\mu^{2}-2 \mu+2\left(\frac{\mu}{\lambda}\right)^{\frac{1}{k}}}}{2 \mu}$. Let $c=f_{\mu}\left(\frac{1}{2}-\frac{1}{2 \sqrt[k]{\mu^{k-1} \lambda}}\right)=\frac{\mu^{\frac{2 k-2}{k}} \lambda^{\frac{2}{k}}-1}{4 \mu^{\frac{k-2}{k}} \lambda^{\frac{2}{k}}}$. Then we have for any $x \in\left[c_{k}, b_{k}\right]$,

$$
\begin{aligned}
{[f_{\lambda} \underbrace{f_{\mu} \cdots f_{\mu}}_{k-1}(x)]_{x}^{\prime} } & <\left(f_{\mu}^{\prime}(m)\right)^{k-2} f_{\mu}^{\prime}\left(\frac{1}{2}-\frac{1}{2 \sqrt[k]{\mu^{k-1} \lambda}}\right) f_{\lambda}^{\prime}(c) \\
& =\left(\mu^{2}-2 \mu+2\left(\frac{\mu}{\lambda}\right)^{\frac{1}{k}}\right)^{\frac{k-2}{2}}\left(\frac{\mu}{\lambda}\right)^{\frac{1}{k}} \lambda\left(1-\frac{\mu^{\frac{2 k-2}{k}} \lambda^{\frac{2}{k}}-1}{2 \mu^{\frac{k-2}{k}} \lambda^{\frac{2}{k}}}\right) \\
& =\left(\mu^{2}-2 \mu+2\left(\frac{\mu}{\lambda}\right)^{\frac{1}{k}}\right)^{\frac{k-2}{2}}\left(\mu^{\frac{1}{k}} \lambda^{\frac{k-1}{k}}-\frac{1}{2} \mu^{\frac{k+1}{k}} \lambda^{\frac{k-1}{k}}+\frac{1}{2}\left(\frac{\lambda}{\mu}\right)^{\frac{k-3}{k}}\right) .
\end{aligned}
$$

Let $E(\lambda)=\left(\mu^{2}-2 \mu+2\left(\frac{\mu}{\lambda}\right)^{\frac{1}{k}}\right)^{\frac{k-2}{2}}\left(\mu^{\frac{1}{k}} \lambda^{\frac{k-1}{k}}-\frac{1}{2} \mu^{\frac{k+1}{k}} \lambda^{\frac{k-1}{k}}+\frac{1}{2}\left(\frac{\lambda}{\mu}\right)^{\frac{k-3}{k}}\right)$ and differentiate $E(\lambda)$ with respect to $\lambda$ to get

$$
E^{\prime}(\lambda)=\left(\mu^{2}-2 \mu+2\left(\frac{\mu}{\lambda}\right)^{\frac{1}{k}}\right)^{\frac{k-2}{2}-1}\left(\frac{\mu}{\lambda}\right)^{\frac{1}{k}} F(\lambda),
$$

where $F(\lambda)=-\frac{k-2}{k} \frac{1}{\lambda}\left(\mu^{\frac{1}{k}} \lambda^{\frac{k-1}{k}}-\frac{1}{2} \mu^{\frac{k+1}{k}} \lambda^{\frac{k-1}{k}}+\frac{1}{2}\left(\frac{\lambda}{\mu}\right)^{\frac{k-3}{k}}\right)+\left(\mu^{2}-2 \mu+2\left(\frac{\mu}{\lambda}\right)^{\frac{1}{k}}\right)\left(\frac{k-1}{k}-\frac{k-1}{2 k} \mu+\right.$ $\left.\frac{k-3}{2 k}\left(\frac{\mu}{\lambda}\right)^{\frac{2}{k}}\right)$, then we have

$$
\begin{aligned}
F(\lambda) & =\left(\mu^{2}-2 \mu\right)\left(\frac{k-1}{k}-\frac{k-1}{2 k} \mu\right)+\left(\frac{\mu}{\lambda}\right)^{\frac{1}{k}} \frac{2-\mu}{2}+\left(\frac{\mu}{\lambda}\right)^{\frac{2}{k}} \frac{k-3}{2 k}(\mu-2)+\frac{k-4}{2 k \mu}\left(\frac{\mu}{\lambda}\right)^{\frac{3}{k}}, \\
F^{\prime}(\lambda) & =-\frac{\mu}{k \lambda^{2}}\left(\frac{\mu}{\lambda}\right)^{\frac{1}{k}-1} G(\lambda),
\end{aligned}
$$

where

$$
G(\lambda)=\frac{2-\mu}{2}+\frac{k-3}{k}(\mu-2)\left(\frac{\mu}{\lambda}\right)^{\frac{1}{k}}+\frac{3(k-4)}{2 k \mu}\left(\frac{\mu}{\lambda}\right)^{\frac{2}{k}}
$$


(i) When $k=3$, since $\lambda \geqslant \frac{\mu}{(2-\mu)^{3}}, \frac{\mu}{\lambda} \leqslant(2-\mu)^{3}$. So we have

$$
G(\lambda)=1-\frac{\mu}{2}-\frac{1}{2 \mu}\left(\frac{\mu}{\lambda}\right)^{\frac{2}{3}} \geqslant 1-\frac{\mu}{2}-\frac{(2-\mu)^{2}}{2 \mu}>0 .
$$

(ii) When $k=4,\left(\frac{\mu}{\lambda}\right)^{\frac{1}{4}}>0.7598$, so

$$
G(\lambda)=1-\frac{\mu}{2}+\frac{1}{4}\left(\frac{\mu}{\lambda}\right)^{\frac{1}{4}}>1-\frac{\mu}{2}+0.1899(\mu-2)>0 .
$$

(iii) When $k \geqslant 5,\left(\frac{\mu}{\lambda}\right)^{\frac{1}{k}} \geqslant\left(\frac{\mu}{\lambda}\right)^{\frac{1}{5}}>0.8$ and $\mu(2-\mu)<1$ for $\mu \in(1,2]$, so

$$
\begin{aligned}
G^{\prime}(\lambda) & =-\frac{\mu}{k^{2} \lambda}\left(\frac{1}{\lambda}\right)^{\frac{1}{k}-1}\left[(k-3) \mu(\mu-2)+3(k-4)\left(\frac{\mu}{\lambda}\right)^{\frac{1}{k}}\right] \\
& <-\frac{\mu}{k^{2} \lambda}\left(\frac{1}{\lambda}\right)^{\frac{1}{k}-1}[\mu(\mu-2)(k-3)+2.4(k-4)] \\
& <-\frac{\mu}{k^{2} \lambda}\left(\frac{1}{\lambda}\right)^{\frac{1}{k}-1}(k-3+2.4 k-9.6)<0 .
\end{aligned}
$$

So when $k \geqslant 5, G(\lambda)$ decreases as $\lambda$ increases. Because $\frac{1.18}{(2-1.18)^{3}}>3$ and $\frac{\mu}{(2-\mu)^{k}} \leqslant \lambda \leqslant 3$, so $\mu<1.18$ when $k \geqslant 3$ and so $\left(\frac{\mu}{3}\right)^{\frac{1}{k}-1}>\left(\frac{1.18}{3}\right)^{\frac{1}{5}-1}>2.1$. Thus we have

$$
\begin{aligned}
G & \geqslant G(3)=\frac{2-\mu}{2}+\frac{k-3}{k}(\mu-2)\left(\frac{\mu}{3}\right)^{\frac{1}{k}}+\frac{3(k-4)}{2 k \mu}\left(\frac{\mu}{3}\right)^{\frac{2}{k}} \\
& =\frac{2-\mu}{2}+\frac{k-3}{2 k}(\mu-2)\left(\frac{\mu}{3}\right)^{\frac{1}{k}}+\frac{2-\mu}{2}+\frac{k-3}{k}(\mu-2)\left(\frac{\mu}{3}\right)^{\frac{1}{k}}+\frac{3(k-4)}{2 k \mu}\left(\frac{\mu}{3}\right)^{\frac{2}{k}} \\
& =\frac{2-\mu}{2}\left[1+\frac{k-3}{k}\left(\frac{\mu}{3}\right)^{\frac{1}{k}}\right]+\left(\frac{\mu}{3}\right)^{\frac{1}{k}} \frac{1}{2 k}\left[(k-3)(\mu-2)+(k-4)\left(\frac{\mu}{3}\right)^{\frac{1}{k}-1}\right] \\
& >\left(\frac{\mu}{3}\right)^{\frac{1}{k}} \frac{1}{2 k}[3-k+2.1(k-4)]>0 .
\end{aligned}
$$

As a result, $G(\lambda)>0$ when $k \geqslant 3$ and so $F^{\prime}(\lambda)<0$. $F(\lambda)$ decreases as $\lambda$ increases.

$$
\begin{aligned}
F(\lambda) & \leqslant F\left(\frac{\mu}{(2-\mu)^{k}}\right)=\frac{(2-\mu)^{2}}{2 k}\left[-(k-1) \mu+k-(k-3)(2-\mu)+\frac{(k-4)(2-\mu)}{\mu}\right] \\
& <\frac{(2-\mu)^{2}}{2 k}[-(k-1) \mu+k+(2-\mu)(k-4-k+3)]<0 .
\end{aligned}
$$

So $F(\lambda)<0$ and $E^{\prime}(\lambda)<0$. $E(\lambda)$ decreases as $\lambda$ increases.

$$
\begin{aligned}
E(\lambda) & \leqslant E\left(\frac{\mu}{(2-\mu)^{k}}\right)=\left(\mu^{2}-2 \mu+2(2-\mu)\right)^{\frac{k-2}{2}}\left(\frac{\mu}{(2-\mu)^{k-1}}-\frac{1}{2} \frac{\mu}{(2-\mu)^{k-1}}+\frac{1}{2}(2-\mu)^{3-k}\right) \\
& =(2-\mu)^{k-2} \frac{1}{(2-\mu)^{k-2}}=1 .
\end{aligned}
$$

Therefore, $[f_{\lambda} \underbrace{f_{\mu} \cdots f_{\mu}}_{k-1}(x)]_{x}^{\prime}<E \leqslant 1$ and Lemma 2.1 .14 is proved. 
Proof of Lemma 2.1.13. We consider two cases $k \in[2,23]$ and $k>23$.

Case (I), $k \in[2,23]$. For this case, there are two subcases: $\lambda \in(2,2.8]$ and $\lambda \in(2.8,3]$. Case(i), $\lambda \in(2,2.8]$. We will prove $(2.32)$ by induction method.

Consider $\lambda \geqslant \frac{\mu}{(2-\mu)^{2}}$. Set $b_{2}$ satisfying $f_{\mu}\left(b_{2}\right)=\frac{1}{2}-\frac{1}{2 \sqrt{\mu \lambda}}$. Then from Lemma 2.1.12, for any $x \in\left[1-\frac{1}{\mu}, b_{2}\right]$,

$$
f_{\lambda}\left(f_{\mu}\left(f_{\mu}(x)\right)\right)>x
$$

Now we assume for any $k \geqslant 3$ and $\lambda \geqslant \frac{\mu}{(2-\mu)^{k-1}}$,

$$
f_{\lambda} \underbrace{f_{\mu} \cdots f_{\mu}}_{k-1}(x)>x, \quad \forall x \in\left[\frac{1}{2}-\frac{1}{2 \sqrt[k]{\mu^{k-1} \lambda}}, b_{k-1}\right],
$$

where $b_{k-1}$ is defined by $\underbrace{f_{\mu} \cdots f_{\mu}}_{k-2}\left(b_{k-1}\right)=\frac{1}{2}-\frac{1}{2 \sqrt[k-1]{\mu^{k-2} \lambda}}$.

First, we will prove the following inequality

$$
f_{\lambda} \underbrace{f_{\mu} \cdots f_{\mu}}_{k}\left(b_{k}\right)>b_{k},
$$

where $b_{k}$ is defined by $\underbrace{f_{\mu} \cdots f_{\mu}}_{k-1}\left(b_{k}\right)=\frac{1}{2}-\frac{1}{2 \sqrt[k]{\mu^{k-2} \lambda}}$.
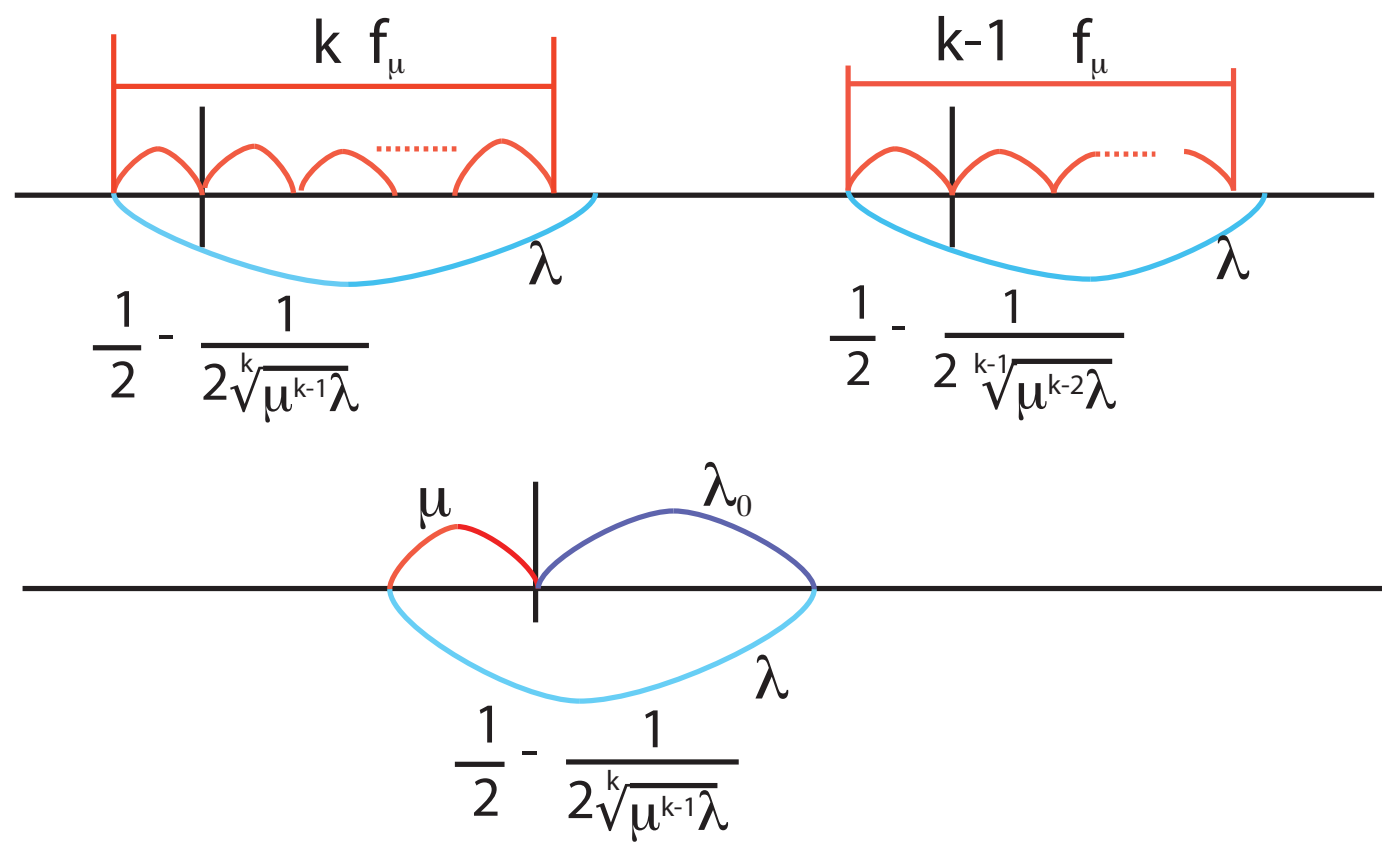

Figure 4.7: $k \in[3,23]$ and $\lambda \in(2,2.8]$ 
When $\lambda \geqslant \frac{\mu}{(2-\mu)^{3}}$, we choose some $\lambda_{0}$ such that

$$
f_{\lambda_{0}}\left(\frac{1}{2}-\frac{1}{2 \sqrt[k]{\mu^{k-1} \lambda}}\right)=f_{\lambda}\left(f_{\mu}\left(\frac{1}{2}-\frac{1}{2 \sqrt[k]{\mu^{k-1} \lambda}}\right)\right) .
$$

It is easy to solve this equation and get

$$
\lambda_{0}=\lambda\left(\mu-\frac{1}{4} \mu^{2}+\frac{1}{4}\left(\frac{\mu}{\lambda}\right)^{\frac{2}{k}}\right) .
$$

Now we will prove

$$
\frac{1}{2}-\frac{1}{2 \sqrt[k]{\mu^{k-1} \lambda}}<f_{\mu}\left(\frac{1}{2}-\frac{1}{2 \sqrt{k-1} \mu^{k-2} \lambda_{0}}\right) .
$$

(4.24) can be simplified into the following inequality,

$$
\left(\lambda-\frac{\mu \lambda}{4}+\frac{1}{4}\left(\frac{\mu}{\lambda}\right)^{\frac{2}{k}-1}\right)\left(\mu^{2}-2 \mu+2\left(\frac{\mu}{\lambda}\right)^{\frac{1}{k}}\right)^{\frac{k-1}{2}}>1 .
$$

Let $A(\mu, \lambda)=\left(\lambda-\frac{\mu \lambda}{4}+\frac{1}{4}\left(\frac{\mu}{\lambda}\right)^{\frac{2}{k}-1}\right)\left(\mu^{2}-2 \mu+2\left(\frac{\mu}{\lambda}\right)^{\frac{1}{k}}\right)^{\frac{k-1}{2}}-1$ and differentiate $A$ with respect to $\mu$ to get

$$
\begin{aligned}
\frac{\partial A}{\partial \mu}= & \left.\left(-\frac{\lambda}{4}+\frac{1}{4}\left(\frac{2}{k}-1\right)\left(\frac{\mu}{\lambda}\right)^{\frac{2}{k}-2}\right) \frac{1}{\lambda}\right)\left(\mu^{2}-2 \mu+2\left(\frac{\mu}{\lambda}\right)^{\frac{1}{k}}\right)^{\frac{k-1}{2}} \\
& +\frac{k-1}{2}\left(\mu^{2}-2 \mu+2\left(\frac{\mu}{\lambda}\right)^{\frac{1}{k}}\right)^{\frac{k-1}{2}-1}\left(2 \mu-2+\frac{2}{k}\left(\frac{\mu}{\lambda}\right)^{\frac{1}{k}-1} \frac{1}{\lambda}\right)\left(\lambda-\frac{\mu \lambda}{4}+\frac{1}{4}\left(\frac{\mu}{\lambda}\right)^{\frac{2}{k}-1}\right) \\
= & \left(\mu^{2}-2 \mu+2\left(\frac{\mu}{\lambda}\right)^{\frac{1}{k}}\right)^{\frac{k-1}{2}-1}\left[\left(-\frac{\lambda}{4}-\frac{1}{4}\left(1-\frac{2}{k}\right)\left(\frac{\mu}{\lambda}\right)^{\frac{2}{k}-1}\right) \frac{1}{\mu}\right)\left(\mu^{2}-2 \mu+2\left(\frac{\mu}{\lambda}\right)^{\frac{1}{k}}\right) \\
& \left.+\left(\lambda-\frac{\mu \lambda}{4}+\frac{1}{4}\left(\frac{\mu}{\lambda}\right)^{\frac{2}{k}-1}\right)\left((k-1)(\mu-1)+\frac{k-1}{k} \frac{1}{\mu}\left(\frac{\mu}{\lambda}\right)^{\frac{1}{k}}\right)\right] .
\end{aligned}
$$

Since

$$
\begin{array}{ll}
\lambda-\frac{\mu \lambda}{4}+\frac{1}{4}\left(\frac{\mu}{\lambda}\right)^{\frac{2}{k}-1}-2\left[\frac{\lambda}{4}+\frac{1}{4 \mu}\left(1-\frac{2}{k}\right)\left(\frac{\mu}{\lambda}\right)^{\frac{2}{k}-1}\right]>\frac{\lambda}{4}\left(2-\mu-\frac{1}{\mu^{2}}\right)>0, \\
\text { and } \left.\quad 2(k-1)(\mu-1)+2 \frac{k-1}{k \mu}\left(\frac{\mu}{\lambda}\right)^{\frac{1}{k}}-\left(\mu^{2}-2 \mu+2\left(\frac{\mu}{\lambda}\right)^{\frac{1}{k}}\right)\right) \\
=(\mu-1)(2(k-1)-(\mu-1))+2\left(\frac{\mu}{\lambda}\right)^{\frac{1}{k}}\left(\frac{k-1}{k \mu}-1\right)+1>-\frac{2}{k}+1>0 .
\end{array}
$$

So $\frac{\partial A}{\partial \mu}>0$ and $A(\mu, \lambda)$ increases as $\mu$ increases. Therefore,

$$
A(\mu, \lambda)>A(1, \lambda)=\left(\frac{3}{4} \lambda+\frac{1}{4} \lambda^{1-\frac{2}{k}}\right)\left(-1+2 \lambda^{-\frac{1}{k}}\right)^{\frac{k-1}{2}}-1=: B(\lambda, k) .
$$

Differentiate $B$ with respect to $\lambda$ to get

$$
\frac{\partial B(\lambda, k)}{\partial \lambda}=\left(-1+2 \lambda^{-\frac{1}{k}}\right)^{\frac{k-1}{2}-1} D(\lambda, k),
$$


where $D(\lambda, k)=-\frac{3}{4}+\frac{3 k+3}{4 k} \lambda^{-\frac{1}{k}}-\frac{k-2}{4 k} \lambda^{-\frac{2}{k}}+\frac{k-3}{4 k} \lambda^{-\frac{3}{k}}$. Now it is obvious that

$$
\frac{\partial D}{\partial \lambda}=\frac{\lambda^{-\frac{1}{k}-1}}{4 k^{2}}\left[-(3 k+3)+(2 k-4) \lambda^{-\frac{1}{k}}-3(k-3) \lambda^{-\frac{2}{k}}\right]<0 .
$$

So $D(\lambda, k)$ decreases as $\lambda$ increases and $D(\lambda, k)<D(2, k)$. Moreover,

$$
\frac{\partial D(2, k)}{\partial k}=\frac{\lambda^{-\frac{3}{k}}}{4 k^{3}}\left[2^{\frac{1}{k}}(-3.4 k+2.8)+2^{\frac{2}{k}}(-0.9 k+2.1)+5.1 k-2.1\right]>0, \quad k \in[5,23] .
$$

So $D(2, k)<D(2,23)<0$. Because $D(2,3)<0$ and $D(2,4)<0$, we can get $D(\lambda, k)<0$ for $k \in[3,23]$. Therefore, $\frac{\partial B(\lambda, k)}{\partial \lambda}<0$ and $B>B(2.8, k)$.

$$
\begin{aligned}
\frac{\partial B(2.8, k)}{\partial k}= & \frac{\left(-1+2 \cdot 2.8^{-\frac{1}{k}}\right)^{\frac{k-1}{2}-1}}{2 k^{2}}\left[2.8^{1-\frac{2}{k}}\left(-1+2 \cdot 2.8^{-\frac{1}{k}}\right) \ln 2.8\right. \\
& \left.+k^{2}\left(2.1+\frac{2.8^{1-\frac{2}{k}}}{4}\right)\left(-1+2 \cdot 2.8^{-\frac{1}{k}}\right) \ln \left(-1+2 \cdot 2.8^{-\frac{1}{k}}\right)+2 \ln 2.8(k-1) \cdot 2.8^{-\frac{1}{k}}\right] \\
:= & \frac{\left(-1+2 \cdot 2.8^{-\frac{1}{k}}\right)^{\frac{k-1}{2}-1}}{2 k^{2}} E,
\end{aligned}
$$

where

$$
\begin{aligned}
E= & \left(-1+2 \cdot 2.8^{-\frac{1}{k}}\right) 2.8^{1-\frac{2}{k}}\left(1.03+0.13 k^{2} \frac{\left.\left(2.1+\frac{2.8^{1-\frac{2}{k}}}{4}\right) \ln \left(-1+2 \cdot 2.8^{-\frac{1}{k}}\right)\right)}{2.8^{1-\frac{2}{k}}}\right. \\
& +2.8^{-\frac{1}{k}}\left[0.87 k^{2} \frac{\left.\left(2.1+\frac{2.8^{1-\frac{2}{k}}}{4}\right)\left(-1+2 \cdot 2.8^{-\frac{1}{k}}\right) \ln \left(-1+2 \cdot 2.8^{-\frac{1}{k}}\right)\right)}{2.8^{-\frac{1}{k}}}+2 \ln 2.8(k-1)\right] .
\end{aligned}
$$

Let $a=2.8^{-\frac{1}{k}}$, then we have $a \leqslant 2.8^{-\frac{1}{23}}$ and

$$
\begin{aligned}
\left(\frac{\left(2.1+0.7 a^{2}\right)(-1+2 a) \ln (-1+2 a)}{a}\right)_{a}^{\prime}>0, & \text { so } \frac{\left(2.1+0.7 a^{2}\right)(-1+2 a) \ln (-1+2 a)}{a}<-0.239, \\
\left(\frac{\left(2.1+0.7 a^{2}\right) \ln (-1+2 a)}{2.8 a^{2}}\right)_{a}^{\prime}>0, & \text { so } \frac{\left(2.1+0.7 a^{2}\right) \ln (-1+2 a)}{2.8 a^{2}}<-0.098 .
\end{aligned}
$$

Now we will prove for $k \in[3,23], E<0$. First, when $k \in[9,23]$,

$$
E<\left(-1+2 \cdot 2.8^{-\frac{1}{k}}\right) 2.8^{1-\frac{2}{k}}\left(1.03-0.01274 k^{2}\right)+2.8^{-\frac{1}{k}}\left(0.20793 k^{2}+2.06 k-2.06\right)<0 .
$$

Second, when $k \in[4,8], \frac{\left(2.1+0.7 a^{2}\right)(-1+2 a) \ln (-1+2 a)}{a}<-0.6298$ and $\frac{\left(2.1+0.7 a^{2}\right) \ln (-1+2 a)}{2.8 a^{2}}<$ -0.337 . We have

$$
E<\left(-1+2 \cdot 2.8^{-\frac{1}{k}}\right) 2.8^{1-\frac{2}{k}}\left(1.03-0.35 \cdot 0.337 k^{2}\right)+2.8^{-\frac{1}{k}}\left(0.65 \cdot 0.6298 k^{2}+2.06 k-2.06\right)<0 .
$$

Finally, when $k=3$, we can also get $E<0$.

So $E<0$ for $k \in[3,23]$. Then we have $\frac{\partial B(2.8, k)}{\partial k}<0$ and

$$
B(\lambda, k)>B(2.8,23)>0
$$


As a result, for $k \in[3,23]$ and $\lambda \in(2,2.8]$, we have $A(\mu, \lambda)>0$ and (4.22) holds.

On the other hand, we will prove (4.21) always holds for $x \in\left[1-\frac{1}{\mu}, b_{k}\right]$.

$$
\begin{aligned}
{[f_{\lambda} \underbrace{f_{\mu} \cdots f_{\mu}}_{k}(x)-x]_{x}^{\prime}=} & f_{\lambda}^{\prime}(\underbrace{f_{\mu} \cdots f_{\mu}}_{k}(x)) \cdot f_{\mu}^{\prime}(\underbrace{f_{\mu} \cdots f_{\mu}}_{k-1}(x)) \cdots f_{\mu}^{\prime}\left(f_{\mu}(x)\right) \cdot f_{\mu}^{\prime}(x)-1, \\
{[f_{\lambda} \underbrace{f_{\mu} \cdots f_{\mu}}_{k}(x)-x]_{x}^{\prime \prime}=} & -2 \lambda \cdot\left[f_{\mu}^{\prime}(\underbrace{f_{\mu} \cdots f_{\mu}}_{k-1}(x)) \cdots f_{\mu}^{\prime}\left(f_{\mu}(x)\right) \cdot f_{\mu}^{\prime}(x)\right]^{2} \\
& -2 \mu f_{\lambda}^{\prime}(\underbrace{f_{\mu} \cdots f_{\mu}}_{k}(x))\left[f_{\mu}^{\prime}(\underbrace{f_{\mu} \cdots f_{\mu}}_{k-1}(x)) \cdots f_{\mu}^{\prime}\left(f_{\mu}(x)\right) \cdot f_{\mu}^{\prime}(x)\right]^{2} \cdots \\
& -2 \mu f_{\lambda}^{\prime}(\underbrace{f_{\mu} \cdots f_{\mu}}_{k}(x)) \cdot f_{\mu}^{\prime}(\underbrace{f_{\mu} \cdots f_{\mu}}_{k-1}(x)) \cdots f_{\mu}^{\prime}\left(f_{\mu}(x)\right) \\
< & 0 .
\end{aligned}
$$

Therefore, $f_{\lambda} \underbrace{f_{\mu} \cdots f_{\mu}}_{k}(x)-x$ gets the minimum value at $x=1-\frac{1}{\mu}$ and $x=b_{k}$. But it is evident that $f_{\lambda} \underbrace{f_{\mu} \cdots f_{\mu}}_{k}\left(1-\frac{1}{\mu}\right)>1-\frac{1}{\mu},(2.32)$ holds for any $x \in\left[1-\frac{1}{\mu}, b_{k}\right], k \in[2,23]$.

Case (ii), $\lambda \in(2.8,3]$. If we let divide $k$ into cases $3 m, 3 m+1$ and $3 m+2$, so $m \in[1,7]$. Firstly, we will prove the following lemma.

Lemma 4.4.1 For any $k \geqslant 3$ and $\lambda \in[2.5,3]$, we have the following inequality

$$
f_{\mu}\left(f_{\mu}\left(f_{\mu}\left(\frac{1}{2}+\frac{1}{2 \sqrt[k]{\mu^{k-1} \lambda}}\right)\right)\right)>\frac{1}{2}-\frac{1}{2 \sqrt[k+2]{\mu^{k+1} \lambda}} .
$$

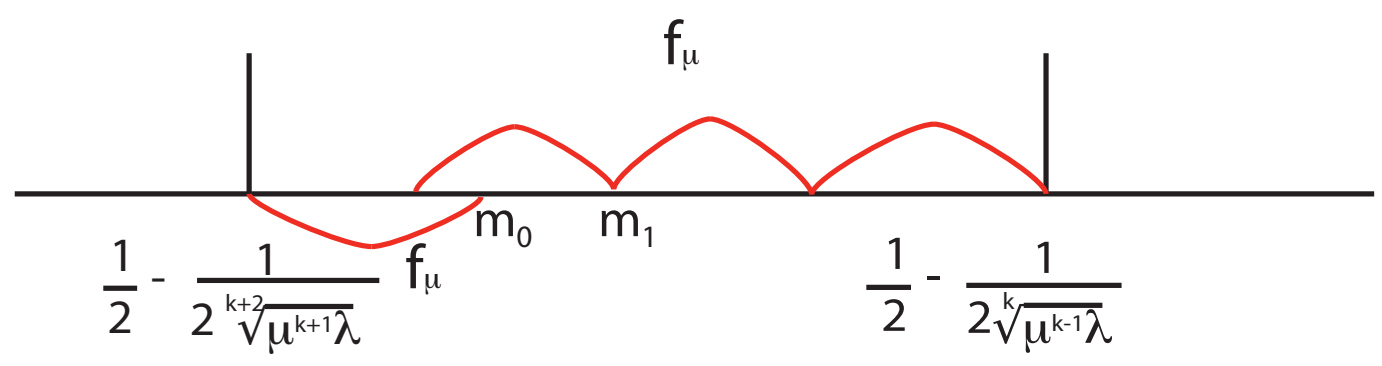

Figure 4.8: $m_{0}<m_{1}$

Proof. Set $m_{0}$ satisfying $f_{\mu}\left(m_{0}\right)=\frac{1}{2}-\frac{1}{2 \sqrt[k+2]{\mu^{k+1} \lambda}}$ and $m_{1}=f_{\mu}\left(f_{\mu}\left(\frac{1}{2}-\frac{1}{2 \sqrt[k]{\mu^{k-1} \lambda}}\right)\right)$. In order to get (4.26), we will prove $m_{0}<m_{1}$, i.e

$$
\frac{\left(\mu^{2}-\left(\frac{\mu}{\lambda}\right)^{\frac{2}{k}}\right)\left(4 \mu-\mu^{2}+\left(\frac{\mu}{\lambda}\right)^{\frac{2}{k}}\right)}{16 \mu}>\frac{\mu-\sqrt{\mu^{2}-2 \mu+2\left(\frac{\mu}{\lambda}\right)^{\frac{1}{k+2}}}}{2 \mu} .
$$

Let $A(\mu)=\left(\mu^{2}-\left(\frac{\mu}{\lambda}\right)^{\frac{2}{k}}\right)\left(4 \mu-\mu^{2}+\left(\frac{\mu}{\lambda}\right)^{\frac{2}{k}}\right)-8 \mu+8 \sqrt{\mu^{2}-2 \mu+2\left(\frac{\mu}{\lambda}\right)^{\frac{1}{k+2}}}$ and differentiate $A(\mu)$ with respect to $\mu$ to get

$$
\frac{1}{4} A^{\prime}(\mu)=3 \mu^{2}-\mu^{3}-2+B(\mu)
$$


where $B(\mu)=(\mu-1)\left(\frac{\mu}{\lambda}\right)^{\frac{2}{k}}+\frac{\mu-2}{k}\left(\frac{\mu}{\lambda}\right)^{\frac{2}{k}}-\frac{1}{k \mu}\left(\frac{\mu}{\lambda}\right)^{\frac{4}{k}}+2 \frac{\mu-1+\frac{1}{(k-2) \mu}\left(\frac{\mu}{\lambda}\right)^{\frac{1}{k+2}}}{\sqrt{\mu^{2}-2 \mu+2\left(\frac{\mu}{\lambda}\right)^{\frac{1}{k+2}}}}$. Firstly, it is easy to see $3 \mu^{2}-\mu^{3}-2>0$ for $\mu \in(1,2]$ and we let. Secondly, we differentiate $B(\mu)$ with respect to $\mu$ and get

$$
\begin{aligned}
& B^{\prime}(\mu)=\left(\frac{\mu}{\lambda}\right)^{\frac{2}{k}}+\frac{2(\mu-1)}{k \mu}\left(\frac{\mu}{\lambda}\right)^{\frac{2}{k}}+\frac{1}{k}\left(\frac{\mu}{\lambda}\right)^{\frac{2}{k}}+\frac{2(\mu-2)}{k^{2} \mu}\left(\frac{\mu}{\lambda}\right)^{\frac{2}{k}}+\frac{1}{k \mu^{2}}\left(\frac{\mu}{\lambda}\right)^{\frac{4}{k}}-\frac{4}{k^{2} \mu^{2}}\left(\frac{\mu}{\lambda}\right)^{\frac{4}{k}} \\
& +2 \frac{\left[1-\frac{1}{(k+2) \mu^{2}}\left(\frac{\mu}{\lambda}\right)^{\frac{1}{k+2}}+\frac{1}{(k+2)^{2} \mu^{2}}\left(\frac{\mu}{\lambda}\right)^{\frac{1}{k+2}}\right]\left(\mu^{2}-2 \mu+2\left(\frac{\mu}{\lambda}\right)^{\frac{1}{k+2}}\right)-\left(\mu-1+\frac{1}{(k+2) \mu}\left(\frac{\mu}{\lambda}\right)^{\frac{1}{k+2}}\right)^{2}}{\left(\mu^{2}-2 \mu+2\left(\frac{\mu}{\lambda}\right)^{\frac{1}{k+2}}\right) \sqrt{\mu^{2}-2 \mu+2\left(\frac{\mu}{\lambda}\right)^{\frac{1}{k+2}}}} .
\end{aligned}
$$

Since $\frac{1.253}{(2-1.253)^{3}}>3$, so we have $\mu<1.253$. Note the following facts for $k \geqslant 3$ :

(i). $\quad\left(\frac{\mu}{\lambda}\right)^{\frac{2}{k}}-\frac{4}{k^{2} \mu^{2}}\left(\frac{\mu}{\lambda}\right)^{\frac{4}{k}}>\left(\frac{\mu}{\lambda}\right)^{\frac{2}{k}}\left(1-\frac{4}{k^{2}}\right)>0$,

(ii). $\quad \frac{1}{k}\left(\frac{\mu}{\lambda}\right)^{\frac{2}{k}}+\frac{2(\mu-2)}{k^{2} \mu}\left(\frac{\mu}{\lambda}\right)^{\frac{2}{k}}>\frac{1}{k^{2}}\left(\frac{\mu}{\lambda}\right)^{\frac{2}{k}}(k-2)>0$,

(iii). $\quad \mu^{2}-2 \mu+2\left(\frac{\mu}{\lambda}\right)^{\frac{1}{k+2}}-\left(\mu-1+\frac{1}{(k+2) \mu}\left(\frac{\mu}{\lambda}\right)^{\frac{1}{k+1}}\right)=(\mu-1)^{2}+\left(2-\frac{1}{(k+2) \mu}\right)^{\left(\frac{\mu}{\lambda}\right)^{\frac{1}{k+2}}-\mu}$

$$
>\left(2-\frac{1}{5}\right) \times 3.0^{-\frac{1}{5}}-1.253>0,
$$

(iv). $\quad 1-\frac{1}{(k+2) \mu^{2}}\left(\frac{\mu}{\lambda}\right)^{\frac{1}{k+2}}-\left(\mu-1+\frac{1}{(k+2) \mu}\left(\frac{\mu}{\lambda}\right)^{\frac{1}{k+1}}\right)>2-\mu-\frac{2}{k+2}>2-1.253-\frac{2}{5}>0$.

Then we can get $B^{\prime}(\mu)>0$ and we have

$$
B(\mu)>B(\mu=1)=\frac{2}{k+2} \frac{\lambda^{-\frac{1}{k+2}}}{\sqrt{-1+2 \lambda^{-\frac{1}{k+2}}}}-\frac{1}{k}\left(\lambda^{-\frac{2}{k}}+\lambda^{-\frac{4}{k}}\right) .
$$

Let $D(k)=2 \lambda^{-\frac{1}{k+2}}-\frac{k+2}{k}\left(\lambda^{-\frac{2}{k}}+\lambda^{-\frac{4}{k}}\right) \sqrt{-1+2 \lambda^{-\frac{1}{k+2}}}$ and we differentiate $D(k)$ as to $k$ to get

$$
\begin{aligned}
D^{\prime}(k)= & \frac{1}{(k+2)^{2}} \lambda^{-\frac{1}{k+2}}\left(2-\frac{k+2}{k}\left(\lambda^{-\frac{2}{k}}+\lambda^{-\frac{4}{k}}\right) \frac{1}{\sqrt{-1+2 \lambda^{-\frac{1}{k+2}}}}\right) \ln \lambda \\
& +\frac{2}{k^{2}}\left(\lambda^{-\frac{2}{k}}+\lambda^{-\frac{4}{k}} \sqrt{-1+2 \lambda^{-\frac{1}{k+2}}}\right)\left(1-\frac{k+2}{k} \ln \lambda\right)-\frac{k+2}{k^{3}} \lambda^{-\frac{4}{k}} \sqrt{-1+2 \lambda^{-\frac{1}{k+2}}} \ln \lambda \\
= & \frac{\lambda^{-\frac{1}{k+2}} \ln \lambda}{(k+2)^{2}}\left[2-\frac{k+2}{k} \frac{\lambda^{-\frac{2}{k}}+\lambda^{-\frac{4}{k}}}{\sqrt{-1+2 \lambda^{-\frac{1}{k+2}}}}-1.5 \frac{(k+2)^{3}}{k^{3}} \lambda^{\frac{-3 k-8}{k(k+2)}} \sqrt{-1+2 \lambda^{-\frac{1}{k+2}}}\right] \\
& +\frac{\lambda^{-\frac{4}{k}}}{k^{2}} \sqrt{-1+2 \lambda^{-\frac{1}{k+2}}}\left[2\left(1+\lambda^{\frac{2}{k}}\right)\left(1-\frac{k+2}{k} \ln \lambda\right)-0.5 \frac{k+2}{k} \ln \lambda\right] .
\end{aligned}
$$

Since $\ln \lambda \leqslant \ln 3<1.1$, so for $k \geqslant 4$, we have

$$
\left(\frac{(k+2)^{3}}{k^{3}} \lambda^{-\frac{3 k+8}{k(k+2)}}\right)_{k}^{\prime}=\frac{k+2}{k^{4}} \lambda^{-\frac{3 k+8}{k(k+2)}}\left(-6 k-12+\ln \lambda\left(3 k+16+\frac{16}{k}\right)\right)
$$




$$
<\frac{k+2}{k^{4}} \lambda^{-\frac{3 k+8}{k(k+2)}}(-2.7 k+5.6+4.4)<0
$$

So $\frac{(k+2)^{3}}{k^{3}} \lambda^{-\frac{3 k+8}{k(k+2)}}$ decreases as $k$ increases and $\frac{(k+2)^{3}}{k^{3}} \lambda^{-\frac{3 k+8}{k(k+2)}}>1$ when $k=4$. However, even when $k=3, \frac{(k+2)^{3}}{k^{3}} \lambda^{-\frac{3 k+8}{k(k+2)}}>1$. So we have

$$
\frac{(k+2)^{3}}{k^{3}} \lambda^{-\frac{3 k+8}{k(k+2)}}>1, \quad(k \geqslant 3)
$$

Considering the following facts for $\lambda \in[2.5,3]$ :

$$
\begin{aligned}
\left(\frac{k+2}{k}\left(\lambda^{-\frac{2}{k}}+\lambda^{-\frac{4}{k}}\right)\right)_{k}^{\prime} & =\frac{2}{k^{2}}\left[-\left(\lambda^{-\frac{2}{k}}+\lambda^{-\frac{4}{k}}\right)+\frac{k+2}{k} \ln \lambda\left(\lambda^{-\frac{2}{k}}+2 \lambda^{-\frac{4}{k}}\right)\right] \\
& >\frac{2}{k^{2}} \lambda^{-\frac{4}{k}}\left[(\ln 2.5-1) \lambda^{\frac{2}{k}}+(2 \ln 2.5-1)\right]>0 .
\end{aligned}
$$

So $\frac{k+2}{k}\left(\lambda^{-\frac{2}{k}}+\lambda^{-\frac{4}{k}}\right)$ increases as $k$ increases and we have

$$
\frac{k+2}{k}\left(\lambda^{-\frac{2}{k}}+\lambda^{-\frac{4}{k}}\right)>\frac{5}{3}\left(3^{-\frac{2}{3}}+3^{-\frac{4}{3}}\right)>1.186 .
$$

when $k \geqslant 5$,

$$
2\left(1+\lambda^{\frac{2}{k}}\right)\left(1-\frac{k+2}{k} \ln \lambda\right)-0.5 \frac{k+2}{k} \ln \lambda<2\left(1+3^{\frac{2}{5}}\right)(1-\ln \lambda)-0.5 \ln \lambda<0 .
$$

While when $k \in[3,4]$,

$$
\begin{aligned}
2\left(1+\lambda^{\frac{2}{k}}\right)\left(1-\frac{k+2}{k} \ln \lambda\right)-0.5 \frac{k+2}{k} \ln \lambda & <2\left(1+3^{\frac{2}{3}}\right)\left(1-\frac{6}{4} \ln \lambda\right)-0.5 \times \frac{6}{4} \ln \lambda \\
& <6.2-10.05 \ln \lambda<0 .
\end{aligned}
$$

As a result of above facts, we can get

$$
D^{\prime}(k)<\frac{\lambda^{-\frac{1}{k+2}} \ln \lambda}{(k+2)^{2}}\left(2-1.186-1.5 \sqrt{-1+2 \times 3^{-\frac{1}{5}}}\right)<0 .
$$

So $D(k)$ decreases as $k$ increases and

$$
D(k)>\lim _{k \rightarrow \infty} D(k)=0 .
$$

Therefore, $B>0$ and $A^{\prime}(\mu)>0 . A(\mu)$ is an increasing function as to $\mu$ and

$$
A(\mu)>A(1)=8 \sqrt{-1+2 \lambda^{-\frac{1}{k+2}}}-2 \lambda^{-\frac{2}{k}}-\lambda^{-\frac{4}{k}}-5=: E(k, \lambda) .
$$

Differentiate $E(k, \lambda)$ with respect to $k$ to get

$$
\frac{\partial E}{\partial k}=\frac{4}{(k+2)^{2} \sqrt{-1+2 \lambda^{-\frac{1}{k+2}}}}\left(2 \lambda^{-\frac{1}{k+2}}-\frac{(k+2)^{2}}{k^{2}}\left(\lambda^{-\frac{2}{k}}+\lambda^{-\frac{4}{k}}\right) \sqrt{-1+2 \lambda^{-\frac{1}{k+2}}}\right) .
$$


Let $F(k)=2 \lambda^{-\frac{1}{k+2}}-\frac{(k+2)^{2}}{k^{2}}\left(\lambda^{-\frac{2}{k}}+\lambda^{-\frac{4}{k}}\right) \sqrt{-1+2 \lambda^{-\frac{1}{k+2}}}$ and differentiate $F$ with respect to $k$ to get

$$
\begin{aligned}
F^{\prime}(k)= & \frac{1}{(k+2)^{2}} \lambda^{-\frac{1}{k+2}} \ln \lambda\left(2-\frac{(k+2)^{2}}{k^{2}} \frac{\lambda^{-\frac{2}{k}}+\lambda^{-\frac{4}{k}}}{\sqrt{-1+2 \lambda^{-\frac{1}{k+2}}}}\right) \\
& +\frac{k+2}{k^{3}}\left(2 \lambda^{-\frac{2}{k}}+4 \lambda^{-\frac{4}{k}}\right) \sqrt{-1+2 \lambda^{-\frac{1}{k+2}}}\left(1-\frac{k+2}{k} \ln \lambda\right)+\frac{k+2}{k^{3}} 2 \lambda^{-\frac{2}{k}} \sqrt{-1+2 \lambda^{-\frac{1}{k+2}}} \\
= & \frac{\lambda^{-\frac{1}{k+2}}}{(k+2)^{2}}\left[\ln \lambda\left(2-\frac{(k+2)^{2}}{k^{2}} \frac{\lambda^{-\frac{2}{k}}+\lambda^{-\frac{4}{k}}}{\sqrt{-1+2 \lambda^{-\frac{1}{k+2}}}}\right)+0.42 \lambda^{\frac{2}{k}} \frac{(k+2)^{3}}{k^{3}} \lambda^{\frac{-3 k-8}{k(k+2)}} \sqrt{-1+2 \lambda^{-\frac{1}{k+2}}}\right] \\
& +\frac{(k+2)^{3}}{k^{3}} \lambda^{-\frac{2}{k}} \sqrt{-1+2 \lambda^{-\frac{1}{k+2}}}\left[\left(2+4 \lambda^{-\frac{2}{k}}\right)\left(1-\frac{k+2}{k} \ln \lambda\right)+1.58\right] .
\end{aligned}
$$

Note we have the following facts:

$$
\begin{gathered}
(i) . \quad \frac{\lambda^{-\frac{2}{k}}+\lambda^{-\frac{4}{k}}}{\left.\sqrt{-1+2 \lambda^{-\frac{1}{k+2}}}\right)_{\lambda}^{\prime}}=\frac{\lambda^{-1}\left[-2\left(\lambda^{-\frac{2}{k}}+2 \lambda^{-\frac{4}{k}}\right)\left(-1+2 \lambda^{-\frac{1}{k+2}}\right)+\frac{k}{k+2} \lambda^{-\frac{1}{k+2}}\left(\lambda^{-\frac{2}{k}}+\lambda^{-\frac{4}{k}}\right)\right]}{k\left(-1+2 \lambda^{-\frac{1}{k+2}}\right)^{\frac{3}{2}}} \\
<\frac{\lambda^{-1}\left[\left(\lambda^{-\frac{2}{k}}+\lambda^{-\frac{4}{k}}\right)\left(-2+5 \lambda^{-\frac{1}{k+2}}\right)\right]}{k\left(-1+2 \lambda^{-\frac{1}{k+2}}\right)^{\frac{3}{2}}}<0 . \\
\begin{aligned}
(i i) .\left(\frac{(k+2)^{2}}{k^{2}}\left(\lambda^{-\frac{2}{k}}+\lambda^{-\frac{4}{k}}\right)\right)_{k}^{\prime} & =\frac{2(k+2)}{k^{3}}\left[\left(-2+\frac{k+2}{k} \ln \lambda\right)\left(\lambda^{-\frac{2}{k}}+\lambda^{-\frac{4}{k}}\right)+\frac{k+2}{k} \lambda^{-\frac{4}{k}} \ln \lambda\right] \\
& <\frac{2(k+2)}{k^{3}} \lambda^{-\frac{4}{k}}\left[\left(-2+\frac{10}{8} 1.1\right)\left(1+2.5^{\frac{2}{8}}\right)+\frac{10}{8} 1.1\right] \\
& <0, \quad(k \geqslant 8) .
\end{aligned}
\end{gathered}
$$

So when $k \geqslant 11$, we have

$$
\ln \lambda\left(2-\frac{(k+2)^{2}}{k^{2}} \frac{\lambda^{-\frac{2}{k}}+\lambda^{-\frac{4}{k}}}{\sqrt{-1+2 \lambda^{-\frac{1}{k+2}}}}\right)>1.1\left(2-\frac{13^{2}}{11^{2}} \frac{3^{-\frac{2}{11}}+3^{-\frac{4}{11}}}{\sqrt{-1+2 \times 3^{-\frac{1}{13}}}}\right)>-0.417 .
$$

Let $G(\lambda, k)=\left(2+4 \lambda^{-\frac{2}{k}}\right)\left(1-\frac{k+2}{k} \ln \lambda\right)$. When $1-\frac{k+2}{k} \ln \lambda<0$, we have

$$
\begin{aligned}
& \frac{\partial G}{\partial k}=\frac{4 \ln \lambda}{k^{2}}\left(2 \lambda^{-\frac{2}{k}}\left(2-\frac{k+2}{k} \ln \lambda\right)+1\right)>\frac{4 \ln \lambda}{k^{2}}\left(2 \lambda^{-\frac{2}{k}}\left(2-\frac{5.5}{3}\right)\right)>0, \\
& \frac{\partial G}{\partial \lambda}=\frac{2}{k \lambda}\left[2 \lambda^{-\frac{2}{k}}\left(2 \frac{k+2}{k} \ln \lambda-k-4\right)-(k+2)\right]<0 .
\end{aligned}
$$

So when $k \geqslant 11, G(\lambda, k) \geqslant G(3,11)>-1.575$.

$$
\begin{aligned}
(i i i) .\left(\lambda^{\frac{2}{k}} \sqrt{-1+2 \lambda^{-\frac{1}{k+2}}}\right)_{k}^{\prime} & =\frac{\lambda^{\frac{2}{k}} \ln \lambda}{k^{2} \sqrt{-1+2 \lambda^{-\frac{1}{k+2}}}}\left[-2\left(-1+2 \lambda^{-\frac{1}{k+2}}\right)+\lambda^{-\frac{1}{k+2}} \frac{k^{2}}{(k+2)^{2}}\right] \\
& <\frac{\lambda^{\frac{2}{k}} \ln \lambda}{k^{2} \sqrt{-1+2 \lambda^{-\frac{1}{k+2}}}}\left(2-3 \lambda^{-\frac{1}{k+2}}\right)<0 .
\end{aligned}
$$


So $\lambda^{\frac{2}{k}} \sqrt{-1+2 \lambda^{-\frac{1}{k+2}}}$ decreases as $k$ increases and we have

$$
\lambda^{\frac{2}{k}} \sqrt{-1+2 \lambda^{-\frac{1}{k+2}}}>1 .
$$

Considering (4.27), we have the following result for $k \geqslant 11$,

$$
F^{\prime}(k)>\frac{\lambda^{-\frac{1}{k+2}}}{(k+2)^{2}}(-0.417+0.42)+\frac{(k+2)^{3}}{k^{3}} \lambda^{-\frac{2}{k}} \sqrt{-1+2 \lambda^{-\frac{1}{k+2}}}(-1.575+1.58)>0 .
$$

Therefore, when $k \geqslant 11, F$ increases as $k$ increases and we can get $F(k)<\lim _{k \rightarrow \infty} F(k)=$ 0 . So $\frac{\partial E}{\partial k}<0$ when $k \geqslant 1$.

On the other hand, for $k \in[3,10]$,

$$
\frac{\partial E}{\partial \lambda}=\frac{4 \lambda^{-1}}{k+2}\left(-2 \frac{\lambda^{-\frac{1}{k+2}}}{\sqrt{-1+2 \lambda^{-\frac{1}{k+2}}}}+\frac{k+2}{k}\left(\lambda^{-\frac{2}{k}}+\lambda^{-\frac{4}{k}}\right)\right) .
$$

It is easy to see that $\frac{x}{\sqrt{-1+2 x}}$ decreases as $x$ increases when $x=\lambda^{-\frac{1}{k+2}}<1$. So $\frac{\lambda^{-\frac{1}{k+2}}}{\sqrt{-1+2 \lambda^{-\frac{1}{k+2}}}}>$ 1. Because of (4.28), we have $\frac{k+2}{k}\left(\lambda^{-\frac{2}{k}}+\lambda^{-\frac{4}{k}}\right)<\frac{12}{10}\left(2.5^{-\frac{2}{10}}+2.5^{-\frac{4}{10}}\right)<1.74$. Then $\frac{\partial E}{\partial \lambda}<0$ and $E>E(k, 3)$. Moreover, when $\lambda=3$ and $k=5,6,7$,

$$
\begin{aligned}
\left(\frac{(k+2)^{2}}{k^{2}}\left(3^{-\frac{2}{k}}+3^{-\frac{4}{k}}\right)\right)_{k}^{\prime} & =\frac{2(k+2)}{k^{3}} 3^{-\frac{4}{k}}\left[\left(-2+1.1 \frac{k+2}{k}\right) \frac{1+3^{-\frac{2}{k}}}{3^{-\frac{2}{k}}}+1.1 \frac{k+2}{k}\right] \\
& <0 .
\end{aligned}
$$

This means $\frac{(k+2)^{2}}{k^{2}}\left(3^{-\frac{2}{k}}+3^{-\frac{4}{k}}\right)$ decreases as $k$ increases for $k \in[5,10]$.

$$
\begin{aligned}
\frac{F(k)}{\sqrt{-1+2 \times 3^{-\frac{1}{k+2}}}} & =\frac{2 \times 3^{-\frac{1}{k+2}}}{\sqrt{-1+2 \times 3^{-\frac{1}{k+2}}}}-\frac{(k+2)^{2}}{k^{2}}\left(3^{-\frac{2}{k}}+3^{-\frac{4}{k}}\right) \\
& <\frac{2 \times 3^{-\frac{1}{7}}}{\sqrt{-1+2 \times 3^{-\frac{1}{7}}}}-\frac{12^{2}}{10^{2}}\left(3^{-\frac{2}{10}}+3^{-\frac{4}{10}}\right)<0 .
\end{aligned}
$$

It is easy to check $F(3)>0$ and $F(4)>0$, so $F(k)>0$ for $k \geqslant 3$. Thus $\frac{\partial E}{\partial k}<0$ and $E$ decreases as $k$ increases. This leads to

$$
A>E(k, \lambda)>\lim _{k \rightarrow \infty} E(k, \lambda)=0 .
$$

So Lemma 4.4.1 is proved.

Second, we will use Lemma 4.4.1 to finish the proof of Lemma 2.1.13 for $k \in[3,23]$. Consider case (1), $k=3 m$, see Figure 4.9 , we will prove the following inequality in order to prove (4.22),

$$
f_{\lambda}\left(f_{\mu}\left(\frac{1}{2}-\frac{1}{2 \sqrt[3 m]{\mu^{3 m-1} \lambda}}\right)\right)>f_{\mu}\left(\frac{1}{2}-\frac{1}{2 \sqrt[m]{\mu^{m-1} \lambda}}\right)
$$




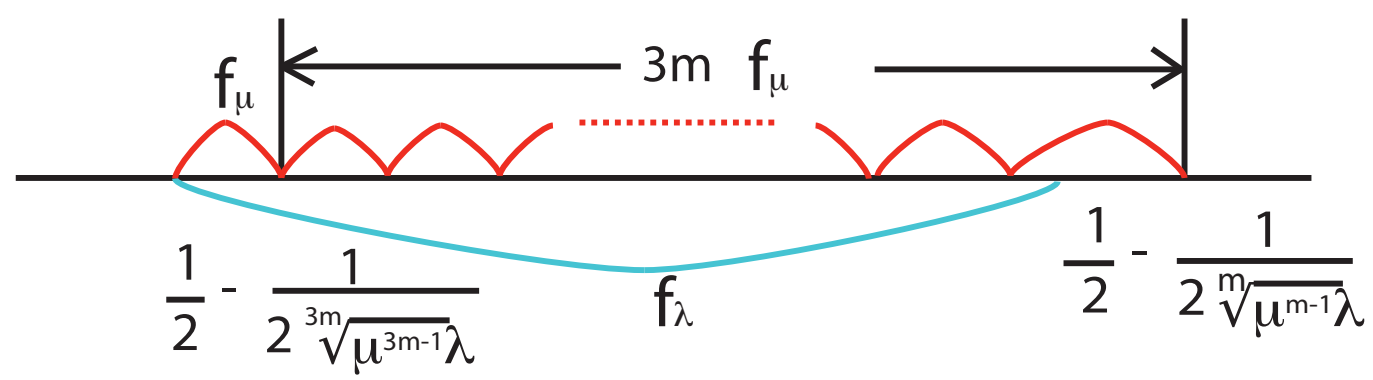

Figure 4.9: $f_{\lambda}\left(f_{\mu}\left(\frac{1}{2}-\frac{1}{2 \sqrt[3 m]{\mu^{3 m-1} \lambda}}\right)\right)>f_{\mu}\left(\frac{1}{2}-\frac{1}{2 \sqrt[m]{\mu^{m-1} \lambda}}\right)$

Which is equivalent to prove

$$
A(\mu, \lambda, m)=\left(\mu^{2}-\left(\frac{\mu}{\lambda}\right)^{\frac{2}{3 m}}\right)\left(4 \mu-\mu^{2}+\left(\frac{\mu}{\lambda}\right)^{\frac{2}{3 m}}\right)-\frac{4 \mu^{3}}{\lambda}+4\left(\frac{\mu}{\lambda}\right)^{1+\frac{2}{m}}>0 .
$$

First note $\frac{1.253}{(2-1.253)^{3}}>3$, so $\mu \in(1,1.253)$. Then we differentiate $A(\mu, \lambda, m)$ with respect to $\mu$ to see that

$$
\begin{aligned}
\frac{1}{2} \frac{\partial A(\mu, \lambda, m)}{\partial \mu}= & 6 \mu^{2}-2 \mu^{3}-\frac{6 \mu^{2}}{\lambda}+(2 \mu-2)\left(\frac{\mu}{\lambda}\right)^{\frac{2}{3 m}} \\
& +\frac{2 \mu}{3 m}\left(\frac{\mu}{\lambda}\right)^{\frac{2}{3 m}}-\frac{2}{3 m \mu}\left(\frac{\mu}{\lambda}\right)^{\frac{4}{3 m}}-\frac{4}{3 m}\left(\frac{\mu}{\lambda}\right)^{\frac{2}{3 m}}+\frac{2(m+2)}{m \lambda}\left(\frac{\mu}{\lambda}\right)^{\frac{2}{m}} \\
> & 2 \mu^{2}\left(3-\frac{3}{2.8}-\mu\right)-\frac{4}{3 m}>2\left(\frac{5.4}{2.8}-1\right)-\frac{4}{3}>0 .
\end{aligned}
$$

So $A(\mu, \lambda, m)$ is an increasing function as to $\mu$ and

$$
\begin{gathered}
A(\mu, \lambda, m)>A(1, \lambda, m)=3-\frac{4}{\lambda}-2 \lambda^{-\frac{2}{3 m}}-\lambda^{-\frac{4}{3 m}}+4 \lambda^{-1-\frac{2}{m}} . \\
\begin{aligned}
\frac{\partial A(1, \lambda, m)}{\partial m} & =\frac{2 \ln \lambda}{3 m^{2}} \lambda^{-1-\frac{2}{m}}\left(\lambda^{\frac{3 m+2}{3 m}}+\lambda^{\frac{3 m+4}{3 m}}-6\right) \\
& >\frac{2 \ln \lambda}{3 m^{2}} \lambda^{-1-\frac{2}{m}}\left(2.8^{\frac{23}{21}}+2.8^{\frac{25}{21}}-6\right)>0 .
\end{aligned}
\end{gathered}
$$

So $A(1, \lambda, m)$ decreases as $m$ increases and we can get

$$
A(\mu, \lambda, m)>A(1, \lambda, 7)=3-\frac{4}{\lambda}-2 \lambda^{-\frac{2}{21}}-\lambda^{-\frac{4}{21}}+4 \lambda^{-\frac{9}{7}} .
$$

On the other hands, we have

$$
\frac{\partial A(1, \lambda, 7)}{\partial \lambda}=\frac{4}{\lambda^{2}}\left(1-\frac{9}{7} \lambda^{-\frac{2}{7}}+\frac{1}{21} \lambda^{\frac{19}{21}}+\frac{1}{21} \lambda^{\frac{17}{21}}\right)>0 .
$$

Therefore, $A(\mu, \lambda, 7)>A(1,2.8,7)$ and (4.29) is proved. So (4.22) holds. Then by (4.25), (4.21) holds for $k \leqslant 23$.

By a similar argument, we can prove (4.21) still holds for $k \leqslant 23$ in the case that $k=3 m+1$ and $k=3 m+2$. 
Case (2). $k=3 m+1$, see Figure 4.10 . We will prove the following inequality:

$$
f_{\lambda}\left(f_{\mu}\left(\frac{1}{2}-\frac{1}{2 \sqrt[3 m+1]{\mu^{3 m} \lambda}}\right)\right)>\frac{1}{2}-\frac{1}{2 \sqrt[m+1]{\mu^{m} \lambda}}
$$

In order to get (4.30), we need to prove

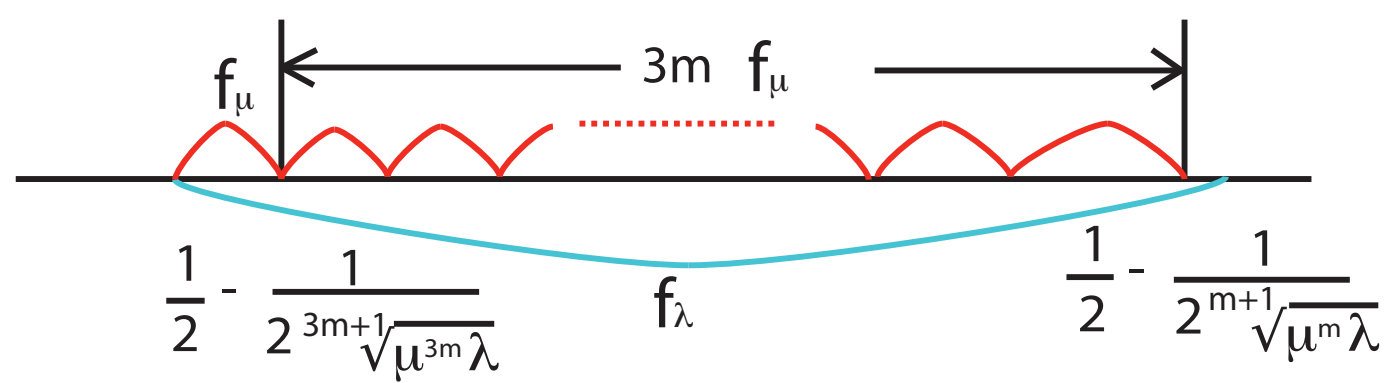

Figure 4.10: $f_{\lambda}\left(f_{\mu}\left(\frac{1}{2}-\frac{1}{2 \sqrt[3 m+1]{\mu^{3 m} \lambda}}\right)\right)>\frac{1}{2}-\frac{1}{2 \sqrt[m+1]{\mu^{m} \lambda}}$

$$
\lambda \frac{\left(\mu^{2}-\left(\frac{\mu}{\lambda}\right)^{\frac{2}{3 m+1}}\right)\left(4 \mu-\mu^{2}+\left(\frac{\mu}{\lambda}\right)^{\frac{2}{3 m+1}}\right)}{16 \mu^{2}}>\frac{1}{2}-\frac{1}{2 \mu}\left(\frac{\mu}{\lambda}\right)^{\frac{1}{m+1}} .
$$

Let $A(\mu, \lambda, m)=\left(\mu^{2}-\left(\frac{\mu}{\lambda}\right)^{\frac{2}{3 m+1}}\right)\left(4 \mu-\mu^{2}+\left(\frac{\mu}{\lambda}\right)^{\frac{2}{3 m+1}}\right)-\frac{8 \mu^{2}}{\lambda}+8\left(\frac{\mu}{\lambda}\right)^{\frac{m+2}{m+1}}$ and differentiate $A(\mu, m)$ with respect to $\mu$ to get

$$
\begin{aligned}
\frac{\partial A(\mu, \lambda, m)}{2 \partial \mu}= & 6 \mu^{2}-2 \mu^{3}-\frac{8 \mu}{\lambda}+(2 \mu-2)\left(\frac{\mu}{\lambda}\right)^{\frac{2}{3 m+1}} \\
& +\frac{2 \mu}{3 m+1}\left(\frac{\mu}{\lambda}\right)^{\frac{2}{3 m+1}}-\frac{2}{(3 m+1) \mu}\left(\frac{\mu}{\lambda}\right)^{\frac{4}{3 m+1}}-\frac{4}{3 m+1}\left(\frac{\mu}{\lambda}\right)^{\frac{2}{3 m+1}}+8 \frac{m+2}{m+1}\left(\frac{\mu}{\lambda}\right)^{\frac{1}{m+1}} \frac{1}{\lambda} .
\end{aligned}
$$

Since $6 \mu^{2}-2 \mu^{3}-\frac{8 \mu}{\lambda}>2 \mu\left(3 \mu-\mu^{2}+2\right)>0$ and $-\frac{4}{3 m+1}\left(\frac{\mu}{\lambda}\right)^{\frac{2}{3 m+1}}+8 \frac{m+2}{m+1}\left(\frac{\mu}{\lambda}\right)^{\frac{1}{m+1}} \frac{1}{\lambda}>$ $8 \cdot 3^{-\frac{3}{2}}-\frac{4}{3}>0, \frac{\partial A(\mu, m)}{\partial \mu}>0$ and we can get

$$
\begin{gathered}
A(\mu, \lambda, m)>A(1, \lambda, m)=3-2 \lambda^{-\frac{2}{3 m+1}}-\lambda^{-\frac{4}{3 m+1}}-\frac{8}{\lambda}+8 \lambda^{-\frac{m+2}{m+1}}, \\
\frac{\partial A(1, \lambda, m)}{\partial m}=-12 \ln \lambda \frac{\lambda^{-\frac{m+2}{m+1}}}{(3 m+1)^{2}}\left(\lambda^{\frac{3 m^{2}+5 m}{(3 m+1)(m+1)}}+\lambda^{\frac{3 m^{2}+3 m-2}{(3 m+1)(m+1)}}-\frac{2(3 m+1)^{2}}{3(m+1)^{2}}\right) .
\end{gathered}
$$

For $m \in[2,7], \frac{2(3 m+1)^{2}}{(m+1)^{2}}<5.042$ and $\lambda^{\frac{3 m^{2}+5 m}{(3 m+1)(m+1)}}+\lambda^{\frac{3 m^{2}+3 m-2}{(3 m+1)(m+1)}}>2.8^{\frac{182}{176}}+2.8^{\frac{166}{176}}>5.54$, so $A(1 . m)$ decreases as $m$ increases and we have

$$
\begin{aligned}
& A(1, \lambda, m)>A(1, \lambda, 7)=3-2 \lambda^{-\frac{2}{22}}-\lambda^{-\frac{4}{22}}-\frac{8}{\lambda}+8 \lambda^{-\frac{9}{8}}, \\
& \frac{\partial A(1, \lambda, 7)}{\partial \lambda}=\lambda^{-2}\left(\frac{4}{22} \lambda^{\frac{20}{22}}+\frac{4}{22} \lambda^{\frac{18}{22}}+8-9 \lambda^{-\frac{1}{8}}\right)>0 .
\end{aligned}
$$


So $A(\mu, \lambda, m)>A(1,2.8,7)>0$ and (4.30) holds. So (4.22) holds and then by (4.25), (4.21) holds for $k \leqslant 23$.

Case (3). $k=3 m+2$, see Figure 4.11, we will prove

$$
f_{\lambda}\left(f_{\mu}\left(\frac{1}{2}-\frac{1}{2 \sqrt[3 m+2]{\mu^{3 m+1} \lambda}}\right)\right)>\frac{1}{2}-\frac{\sqrt{\mu^{2}-2 \mu+2\left(\frac{\mu}{\lambda}\right)^{\frac{1}{m+2}}}}{2 \mu} .
$$

In order to get (4.31), we need to prove

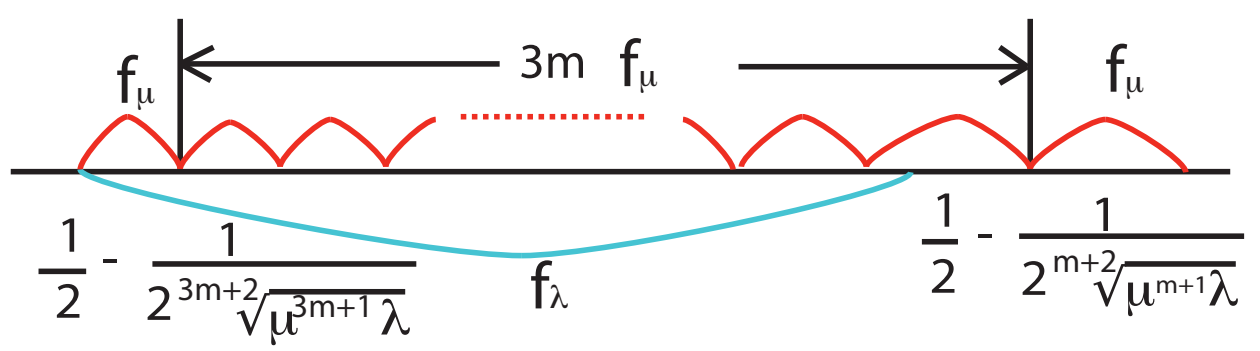

Figure 4.11: $f_{\lambda}\left(f_{\mu}\left(\frac{1}{2}-\frac{1}{2 \sqrt[3 m+2]{\mu^{3 m+1} \lambda}}\right)\right)>\frac{1}{2}-\frac{\sqrt{\mu^{2}-2 \mu+2\left(\frac{\mu}{\lambda}\right)^{\frac{1}{m+2}}}}{2 \mu}$

$$
\lambda \frac{\left(\mu^{2}-\left(\frac{\mu}{\lambda}\right)^{\frac{2}{3 m+2}}\right)\left(4 \mu-\mu^{2}+\left(\frac{\mu}{\lambda}\right)^{\frac{2}{3 m+2}}\right)}{16 \mu^{2}}>\frac{1}{2}-\frac{\sqrt{\mu^{2}-2 \mu+2\left(\frac{\mu}{\lambda}\right)^{\frac{1}{m+2}}}}{2 \mu} .
$$

Let $A(\mu, \lambda, m)=\left(\mu^{2}-\left(\frac{\mu}{\lambda}\right)^{\frac{2}{3 m+2}}\right)\left(4 \mu-\mu^{2}+\left(\frac{\mu}{\lambda}\right)^{\frac{2}{3 m+2}}\right)-\frac{8 \mu^{2}}{\lambda}+\frac{8 \mu}{\lambda} \sqrt{\mu^{2}-2 \mu+2\left(\frac{\mu}{\lambda}\right)^{\frac{1}{m+2}}}$.

$$
\begin{aligned}
\frac{\partial A(\mu, \lambda, m)}{2 \partial \mu}= & 6 \mu^{2}-2 \mu^{3}-\frac{8 \mu}{\lambda}+(2 \mu-2)\left(\frac{\mu}{\lambda}\right)^{\frac{2}{3 m+2}}+\frac{2 \mu}{3 m+2}\left(\frac{\mu}{\lambda}\right)^{\frac{2}{3 m+2}}-\frac{2}{(3 m+2) \mu}\left(\frac{\mu}{\lambda}\right)^{\frac{4}{3 m+2}} \\
& -\frac{4}{3 m+2}\left(\frac{\mu}{\lambda}\right)^{\frac{2}{3 m+2}}+\frac{4}{\lambda} \sqrt{\mu^{2}-2 \mu+2\left(\frac{\mu}{\lambda}\right)^{\frac{1}{m+2}}}+\frac{4 \mu}{\lambda} \cdot \frac{\mu-1+\frac{1}{(m+2) \mu}\left(\frac{\mu}{\lambda}\right)^{\frac{1}{m+2}}}{\sqrt{\mu^{2}-2 \mu+2\left(\frac{\mu}{\lambda}\right)^{\frac{1}{m+2}}}} .
\end{aligned}
$$

Since $\frac{4}{\lambda} \sqrt{\mu^{2}-2 \mu+2\left(\frac{\mu}{\lambda}\right)^{\frac{1}{m+2}}}>\frac{4}{3} \sqrt{-1+2\left(\frac{1}{3}\right)^{\frac{1}{3}}}>0.829>\frac{4}{3 m+2}$ and we recall that $6 \mu^{2}-$ $2 \mu^{3}-\frac{8 \mu}{\lambda}>0$, we can easily get $\frac{\partial A(\mu, \lambda, m)}{\partial \mu}>0$ and

$$
\begin{gathered}
A(\mu, \lambda, m)>A(1, \lambda, m)=3-2 \lambda^{-\frac{2}{3 m+2}}-\lambda^{-\frac{4}{3 m+2}}-\frac{8}{\lambda}+\frac{8}{\lambda} \sqrt{-1+2 \lambda^{-\frac{1}{m+2}}} . \\
\frac{\partial A(1, \lambda, m)}{\partial m}=-\frac{12 \lambda^{-1-\frac{1}{m+2}} \ln \lambda}{(3 m+2)^{2} \sqrt{-1+2 \lambda^{-\frac{1}{m+2}}}}\left[\lambda^{\frac{3 m^{2}+9 m+2}{(3 m+2)(m+2)}} \frac{1+\lambda^{-\frac{2}{3 m+2}}}{\sqrt{-1+2 \lambda^{-\frac{1}{m+2}}}}-\frac{2(3 m+2)^{2}}{3(m+2)^{2}}\right] \\
<-\frac{12 \lambda^{-1-\frac{1}{m+2}} \ln \lambda}{(3 m+2)^{2} \sqrt{-1+2 \lambda^{-\frac{1}{m+2}}}}\left[\lambda\left(1+2.8^{-\frac{2}{5}}\right)-\frac{3 \cdot 23^{2}}{3 \cdot 9^{2}}\right]<0 .
\end{gathered}
$$


So $A(1, \lambda, m)$ decreases as $m$ increases and

$$
\begin{gathered}
A(1, \lambda, m) \geqslant A(1, \lambda, 7)=3-2 \lambda^{-\frac{2}{23}}-\lambda^{-\frac{4}{23}}-\frac{8}{\lambda}+\frac{8}{\lambda} \sqrt{-1+2 \lambda^{-\frac{1}{9}}} \\
\frac{\partial A(1, \lambda, 7)}{\partial \lambda}=\frac{4}{23 \lambda^{2}}\left(\lambda^{\frac{21}{23}}+\lambda^{\frac{19}{23}}\right)+\frac{8}{\lambda^{2} \sqrt{-1+2 \lambda^{-\frac{1}{9}}}}\left(1-\sqrt{-1+2 \lambda^{-\frac{1}{9}}}-\frac{1}{9} \lambda^{-\frac{1}{9}}\right) \\
>\frac{8}{\lambda^{2} \sqrt{-1+2 \lambda^{-\frac{1}{9}}}}\left(1-\sqrt{-1+2 \cdot 2.8^{-\frac{1}{9}}}-\frac{1}{9} \cdot 2.8^{-\frac{1}{9}}\right)>0 .
\end{gathered}
$$

So $A(1, \lambda, 7)$ increases as $\lambda$ increases and

$$
A(\mu, \lambda, m)>A(1,2.8,7)>0 .
$$

So (4.22) holds. With the same reason as the case $\lambda \in(2.2 .8]$. (2.32) can be proved.

Case (II), $k \geqslant 24$. There are two subcases, $\lambda \in(2,2.61]$ and $\lambda \in(2.61,3]$.

Case(i). $\lambda \in(2,2.61]$.

At first, we need to prove the following lemma.

Lemma 4.4.2 When $\lambda \in(2,2.61]$ and $k \geqslant 24$, we have the following inequality

$$
f_{\mu}\left(f_{\mu}\left(\frac{1}{2}-\frac{1}{2 \sqrt[k-1]{\mu^{k-2} \lambda}}\right)\right) \geqslant \frac{1}{2}-\frac{1}{2 \sqrt[k]{\mu^{k-1} \lambda}} .
$$

See Figure 4.12.

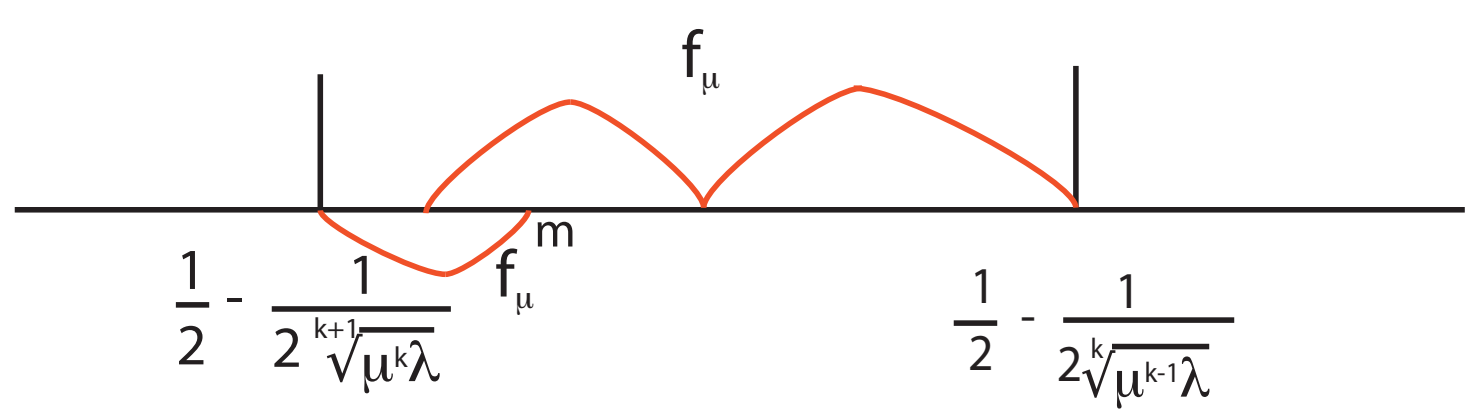

Figure 4.12: $f_{\mu}\left(f_{\mu}\left(\frac{1}{2}-\frac{1}{2 \sqrt[k-1]{\mu^{k-2} \lambda}}\right)\right) \geqslant \frac{1}{2}-\frac{1}{2 \sqrt[k]{\mu^{k-1} \lambda}}$

Proof. Set $m$ satisfying $f_{\mu}(m)=\frac{1}{2}-\frac{1}{2 \sqrt[k+1]{\mu^{k} \lambda}}$. We need to find the condition for $f_{\mu}\left(\frac{1}{2}-\right.$ $\left.\frac{1}{2 \sqrt[k]{\mu^{k-1} \lambda}}\right)>m$. Let $A(\mu, \lambda, k)=\mu^{2}-\left(\frac{\mu}{\lambda}\right)^{\frac{2}{k}}-2 \mu+2 \sqrt{\mu^{2}-2 \mu+2\left(\frac{\mu}{\lambda}\right)^{\frac{1}{k+1}}}$ and then we can get

$$
\frac{\partial A(\mu, \lambda, k)}{\partial \mu}=2\left(\mu-1+\frac{\mu-1+\frac{1}{(k+1) \mu}\left(\frac{\mu}{\lambda}\right)^{\frac{1}{k+1}}}{\sqrt{\mu^{2}-2 \mu+2\left(\frac{\mu}{\lambda}\right)^{\frac{1}{k+1}}}}-\frac{1}{k \mu}\left(\frac{\mu}{\lambda}\right)^{\frac{2}{k}}\right) .
$$


Let $B=\left(\mu-1+\frac{1}{(k+1) \mu}\left(\frac{\mu}{\lambda}\right)^{\frac{1}{k+1}}\right)^{2}-\left(\frac{1}{k \mu}\left(\frac{\mu}{\lambda}\right)^{\frac{2}{k}}\right)^{2}\left(\mu^{2}-2 \mu+2\left(\frac{\mu}{\lambda}\right)^{\frac{1}{k+1}}\right)$, then we have

$$
\begin{aligned}
B=\quad & (\mu-1)^{2}+\frac{\mu-1}{\mu}\left(\frac{\mu}{\lambda}\right)^{\frac{1}{k+1}}\left(\frac{2}{k+1}-\frac{\mu-1}{k^{2} \mu}\left(\frac{\mu}{\lambda}\right)^{\frac{3 k+4}{k(k+1)}}\right) \\
& +\frac{1}{(k+1)^{2} \mu^{2}}\left[\left(\frac{\mu}{\lambda}\right)^{\frac{2}{k+1}}-\frac{(k+1)^{2}}{k^{2}}\left(\frac{\mu}{\lambda}\right)^{\frac{4}{k}}\left(2\left(\frac{\mu}{\lambda}\right)^{\frac{1}{k+1}}-1\right)\right] .
\end{aligned}
$$

Since $\left(\frac{k+1}{k}\left(\frac{\mu}{\lambda}\right)^{\frac{2}{k}}\right)_{k}^{\prime}=\frac{2\left(\frac{\mu}{\lambda}\right)^{\frac{2}{k}}}{k^{2}}\left(-1-2 \frac{k+1}{k} \ln \left(\frac{\mu}{\lambda}\right)\right)>0$, so we have $\frac{k+1}{k}\left(\frac{\mu}{\lambda}\right)^{\frac{2}{k}}<1$. It is also obvious that $\left(\frac{\mu}{\lambda}\right)^{\frac{2}{k+1}}>2\left(\frac{\mu}{\lambda}\right)^{\frac{1}{k+1}}-1$ and $\frac{2}{k+1}-\frac{\mu-1}{k^{2} \mu}\left(\frac{\mu}{\lambda}\right)^{\frac{3 k+4}{k(k+1)}}>0$. It turns over that $B>0$ and $\frac{\partial A(\mu, k)}{\partial \mu}>0$. So $A(\mu, \lambda, k)>A(1, \lambda, k)=2 \sqrt{-1+2 \lambda^{-\frac{1}{k+1}}}-\lambda^{-\frac{2}{k}}-1$.

$$
\begin{aligned}
\left(\frac{k+1}{k}\left(-1+2 \lambda^{-\frac{1}{k+1}}\right)\right)_{k}^{\prime} & =\frac{1}{k^{2}}\left(1-\lambda^{-\frac{1}{k+1}}+2 \frac{k+1}{k} \lambda^{-\frac{1}{k+1}} \ln \lambda\right)>0, \\
\frac{k+1}{k}\left(-1+2 \lambda^{-\frac{1}{k+1}}\right) & <1, \\
\frac{\partial A(1, \lambda, k)}{\partial \lambda} & =\frac{2 \lambda^{-1-\frac{2}{k}}}{k+1}\left(-1+\frac{k+1}{k} \sqrt{-1+2 \lambda^{-\frac{1}{k+1}}}\right)<0 .
\end{aligned}
$$

So $A(1, \lambda, k)$ decreases as $\lambda$ increases. Therefore, for $\lambda \in(2,2.61]$, we have

$$
A(1, \lambda, k)>A(1,2.61, k)
$$

We differentiate $A(1, \lambda, k)$ with respect to $k$ to get

$$
\begin{aligned}
\frac{\partial A(1, \lambda, k)}{\partial k}= & \frac{2 \lambda^{-\frac{2}{k}} \ln \lambda}{(k+1)^{2}}\left(\frac{\lambda^{\frac{k+2}{k(k+1)}}}{\sqrt{-1+2 \lambda^{-\frac{1}{k+1}}}}-\frac{(k+1)^{2}}{k^{2}}\right)=: \frac{2 \lambda^{-\frac{2}{k}} \ln \lambda}{(k+1)^{2}} D(\lambda, k), \\
\frac{\partial D(\lambda, k)}{\partial k}= & \frac{1}{(k+1)^{2}}\left[\frac{\lambda^{\frac{k+2}{k(k+1)}} \ln \lambda\left(1-3 \lambda^{-\frac{1}{k+1}}\right)}{\left(-1+2 \lambda^{-\frac{1}{k+1}}\right)^{\frac{3}{2}}}-\frac{\frac{4 k+2}{k^{2}} \lambda^{\frac{k+2}{k(k+1)}} \ln \lambda}{\sqrt{-1+2 \lambda^{-\frac{1}{k+1}}}}+2 \frac{(k+1)^{3}}{k^{3}}\right] \\
= & : \frac{1}{(k+1)^{2}} E(\lambda, k) . \\
\frac{\partial E(\lambda, k)}{\partial k}= & \frac{\lambda^{\frac{k+2}{k(k+1)}}(\ln \lambda)^{2}}{(k+1)^{2}\left(-1+2 \lambda^{-\frac{1}{k+1}}\right)^{\frac{3}{2}}}\left(\frac{k^{2}+4 k+2}{k^{2}}\left(-1+2 \lambda^{-\frac{1}{k+1}}\right)+\lambda^{-\frac{1}{k+1}}\right) \\
& \left(\frac{-1+3 \lambda^{-\frac{1}{k+1}}}{\left.-1+2 \lambda^{-\frac{1}{k+1}}+\frac{4 k+2}{k^{2}}\right)}\right) \\
& +\frac{\lambda^{\frac{2}{k}}(\ln \lambda)^{2}}{(k+1)^{2}\left(-1+2 \lambda^{-\frac{1}{k+1}}\right)^{\frac{5}{2}}}+\frac{\lambda^{\frac{k+2}{k(k+1)}} \ln \lambda}{\left(-1+2 \lambda^{-\frac{1}{k+1}}\right)^{\frac{1}{2}}} \cdot \frac{4 k+4}{k^{3}}-\frac{6(k+1)^{2}}{k^{4}} \\
> & \frac{1}{k^{2}}\left[\frac{k^{2} \lambda^{\frac{k+2}{k(k+1)}}(\ln \lambda)^{2}}{(k+1)^{2}\left(-1+2 \lambda^{-\frac{1}{k+1}}\right)^{\frac{5}{2}}}\left(\left(3 \lambda^{-\frac{1}{k+1}}-1\right)^{2}+\lambda^{-\frac{1}{k+1}}\right)\right.
\end{aligned}
$$




$$
\left.+\frac{\lambda^{\frac{k+2}{k(k+1)}} \ln \lambda}{\left(-1+2 \lambda^{-\frac{1}{k+1}}\right)^{\frac{1}{2}}} \cdot \frac{4 k+4}{k}-\frac{6(k+1)^{2}}{k^{2}}\right] .
$$

Because $\lim _{k \rightarrow \infty} A(1, k)=0$, so to prove $A(1, k)>0$, we only need to prove $\frac{\partial A(1, k)}{\partial k}<0$, i.e. $D(k)>0$. For $\lambda \in(2,2.61]$ and $k \geqslant 24, \sqrt{-1+2 \lambda^{-\frac{1}{k+1}}}>0.96, \lambda^{\frac{k+2}{k(k+1)}}<1.043$, $\lambda^{-\frac{1}{k+1}}>0.962$ and $\ln \lambda<0.96$. We can verify

$$
\begin{aligned}
& \left(\frac{k+1}{k}\left(-1+2 \lambda^{-\frac{1}{k+1}}\right)\right)_{k}^{\prime}=\frac{1}{k^{2}}\left(1-2 \lambda^{-\frac{1}{k+1}}+\frac{2 k}{k+1} \lambda^{-\frac{1}{k+1}} \ln \lambda\right)>0, \\
& \frac{k+1}{k}\left(-1+2 \lambda^{-\frac{1}{k+1}}\right)<1, \\
& \left(3 \lambda^{-\frac{1}{k+1}}-1\right)^{2}+\lambda^{-\frac{1}{k+1}}=9 \lambda^{-\frac{2}{k+1}}-5 \lambda^{-\frac{1}{k+1}}+1>4.52 .
\end{aligned}
$$

It turns out that

$$
\frac{\partial E(\lambda, k)}{\partial k}>\frac{1}{k^{2}}\left[4.52 \cdot(0.96)^{2}+4-6 \cdot 1.042^{2}\right]>0 .
$$

So $E(2.61, k)$ increases as $k$ increases and it is easy to see

$$
\begin{aligned}
\lim _{k \rightarrow \infty} E(2.61, k) & =-2 \ln \lambda+2>0, \\
A(2.61,24) & =-0.0387<0 .
\end{aligned}
$$

So there exists $k_{1}$ such that $E(2.61, k)<0$ for $k \in\left[24, k_{1}\right]$ and $E(2.61, k)>0$ for $k \geqslant$ $k_{1}$. That is to say $D(2.61, k)$ decreases first and then increases as $k$ increases. Because $\lim _{k \rightarrow \infty} D(2.61, k)=0$ and $D(2.61,24)<0$, we can get $D(2.61, k)<0$ and so $A(1,2.61 . k)$ is a decreasing function as to $k$ when $\lambda \in(2,2.61]$. As a result, we can get

$$
A(\mu, \lambda, k)>\lim _{k \rightarrow \infty} A(1,2.61 . k)=0, \quad \lambda \in(2,2.61], \quad k \geqslant 24 .
$$

Lemma 4.4.2 is proved. Now we will prove Lemma 2.1 .13 when $\lambda \in(2,2.61]$ and $k \geqslant 24$ by induction method.

Consider $\lambda \geqslant \frac{\mu}{(2-\mu)^{22}}$. Set $b_{22}$ satisfying $\underbrace{f_{\mu} \cdots f_{\mu}}_{21}\left(b_{22}\right)=\frac{1}{2}-\frac{1}{2 \sqrt[22]{\mu^{21} \lambda}}$ an set $b_{23}$ satisfying $\underbrace{f_{\mu} \cdots f_{\mu}}_{22}\left(b_{23}\right)=\frac{1}{2}-\frac{1}{2 \sqrt[23]{\mu^{22} \lambda}}$, then we know from the previous section that for any $x \in$ $\left[1-\frac{1}{\mu}, b_{22}\right]$

$$
f_{\lambda}(\underbrace{f_{\mu} \cdots f_{\mu}}_{22}(x))>x,
$$

and for any $x \in\left[1-\frac{1}{\mu}, b_{23}\right]$,

$$
f_{\lambda}(\underbrace{f_{\mu} \cdots f_{\mu}}_{23}(x))>x .
$$

Now we assume for any $k \geqslant 24$, when $\lambda \geqslant \frac{\mu}{(2-\mu)^{k-2}}$,

$$
f_{\lambda} \underbrace{f_{\mu} \cdots f_{\mu}}_{k-2}(x)>x, \quad x \in\left[1-\frac{1}{\mu}, b_{k-2}\right],
$$


where $b_{k-2}$ is defined by $\underbrace{f_{\mu} \cdots f_{\mu}}_{k-3}\left(b_{k-2}\right)=\frac{1}{2}-\frac{1}{2 \sqrt[k-2]{\mu^{k-3} \lambda}}$.

Then we will prove the following inequality as Figure 4.13 shows,

$$
f_{\lambda} \underbrace{f_{\mu} \cdots f_{\mu}}_{k}\left(b_{k}\right)>b_{k}
$$

We can get that $m_{0}=\frac{1}{2}-\frac{\sqrt{\mu^{2}-2 \mu+2\left(\frac{\mu}{\lambda}\right)^{\frac{1}{k}}}}{2 \mu}$ if $f_{\mu}\left(m_{0}\right)=\frac{1}{2}-\frac{1}{2 \sqrt[k]{\mu^{k-1} \lambda}}$, we choose $\lambda_{0}$ such
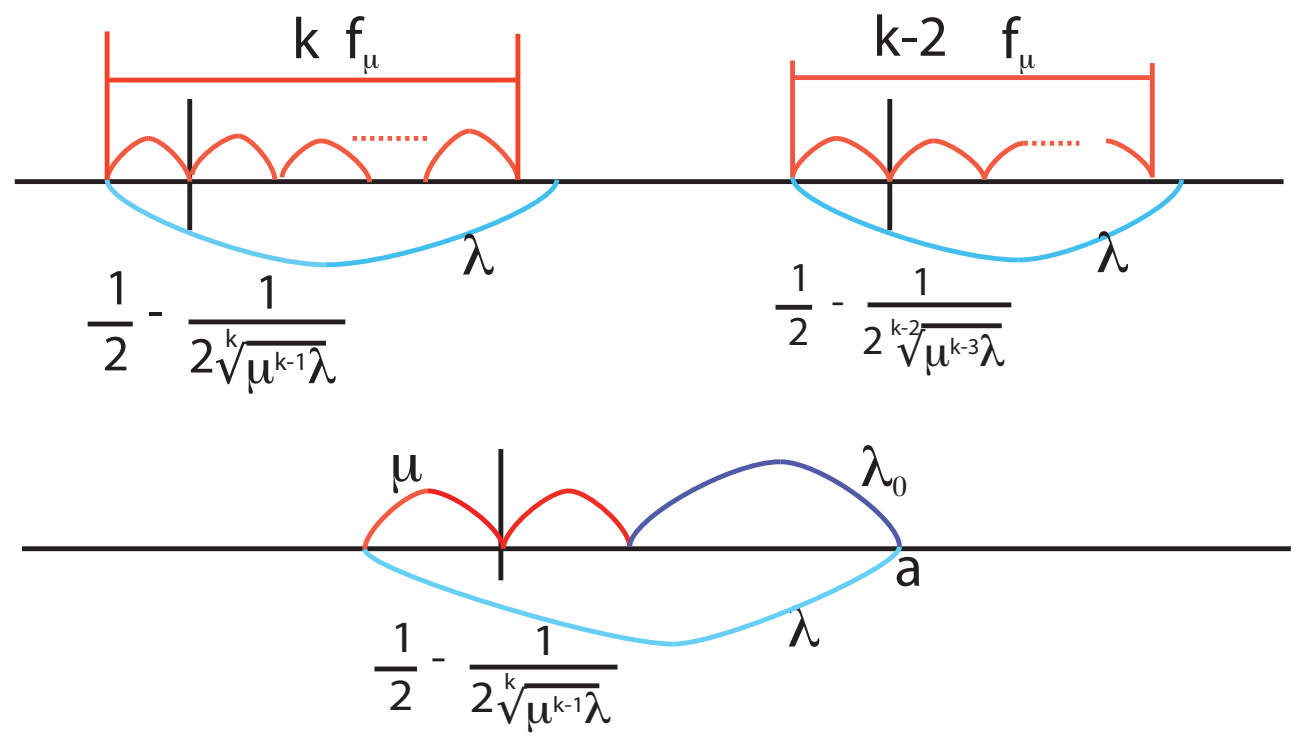

Figure 4.13: $f_{\lambda_{0}}(a)=f_{\lambda}\left(f_{\mu}\left(\frac{1}{2}-\frac{1}{2 \sqrt[k]{\mu^{k-1} \lambda}}\right)\right)$ when $k \geqslant 24$ and $\lambda \in(2,2.61]$ that

$$
f_{\lambda_{0}}(a)=m_{0},
$$

where $a=f_{\lambda}\left(f_{\mu}\left(\frac{1}{2}-\frac{1}{2 \sqrt[k]{\mu^{k-1} \lambda}}\right)\right)$. See Figure 4.13. We can solve $\lambda_{0}$ to get

$$
\lambda_{0}(\lambda)=\frac{\lambda}{8}\left(\frac{\mu}{\lambda}\right)^{\frac{3}{k}}\left(\mu^{\frac{k-1}{k}} \lambda^{\frac{1}{k}}+1\right)\left(4 \mu^{\frac{k-2}{k}} \lambda^{\frac{2}{k}}-\mu^{\frac{2 k-2}{k}} \lambda^{\frac{2}{k}}+1\right) .
$$

(i). We now prove $m_{0}<f_{\mu}\left(\frac{1}{2}-\frac{1}{2 \sqrt[k-2]{\mu^{k-3} \lambda_{0}}}\right)$.

First, we prove

$$
\frac{1}{2}-\frac{1}{2 \sqrt[k]{\mu^{k-1} \lambda}}<\frac{1}{2}-\frac{1}{2 \sqrt[k-1]{\mu^{k-2} \lambda_{0}}}
$$

In order to get (4.35), we need to prove the following inequality,

$$
A(k):=\left(\mu^{\frac{k-1}{k}} \lambda^{\frac{1}{k}}+1\right)\left(4 \mu-\mu^{2}+\left(\frac{\mu}{\lambda}\right)^{\frac{2}{k}}\right)-8>0 .
$$


Differentiate $A$ with respect to $k$ to get

$$
\begin{aligned}
A^{\prime}(k) & \left.\left.\left.=-\frac{1}{k^{2}}\left(\ln \frac{\lambda}{\mu}\right)\left(\frac{\mu}{\lambda}\right)^{\frac{1}{k}}\right)\left[4 \mu^{2}-\mu^{3}-\mu\left(\frac{\mu}{\lambda}\right)^{\frac{2}{k}}\right)-2\left(\frac{\mu}{\lambda}\right)^{\frac{3}{k}}\right)\right] \\
& \left.<-\frac{1}{k^{2}} \ln \frac{\lambda}{\mu}\left(\frac{\mu}{\lambda}\right)^{\frac{1}{k}}\right)\left(4 \mu^{2}-\mu^{3}-\mu-2\right) .
\end{aligned}
$$

Because $\frac{\mu}{(2-\mu)^{2}} \leqslant 3$, we have $\mu \leqslant \frac{4}{3}$ and $\left(4 \mu^{2}-\mu^{3}-\mu-2\right)^{\prime \prime}=8-6 \mu \geqslant 0$. So $4 \mu^{2}-\mu^{3}-\mu-2$ reaches the minimum value at $\mu=1$ and $\mu=\frac{4}{3}$ and thus $4 \mu^{2}-\mu^{3}-\mu-2>0$. As a result, $A^{\prime}(k)<0$ and $A(k)$ decreases as $k$ increases. So

$$
A>\lim _{k \rightarrow \infty} A=(\mu+1)\left(4 \mu-\mu^{2}+1\right)-8=D(\mu) .
$$

Note

$$
D^{\prime}(\mu)=-3 \mu^{2}+6 \mu+5=-3(\mu-1)^{2}+8>0 .
$$

So $D(\mu)$ increases as $\mu$ increases, $D(\mu)>D(1)=0$, so $A>0$. We have proved (4.35). If $f_{\mu}(d)=\frac{1}{2}-\frac{1}{2 \sqrt[k-1]{\mu^{k-2} \lambda_{0}}}$, then $m_{0}<d$. Because $\lambda_{0}<\lambda \in(2,2.61]$, we have $f_{\mu}\left(f_{\mu}\left(\frac{1}{2}-\right.\right.$ $\left.\left.\frac{1}{2 \sqrt[k-2]{\mu^{k-3} \lambda_{0}}}\right)\right)>\frac{1}{2}-\frac{1}{2 \sqrt[k-1]{\mu^{k-2} \lambda_{0}}}$ by Lemma 4.4.2. Therefore,

$$
m_{0}<d<f_{\mu}\left(\frac{1}{2}-\frac{1}{2 \sqrt[k-2]{\mu^{k-3} \lambda}}\right) .
$$

(ii). We now prove $\lambda_{0}>\frac{\mu}{(2-\mu)^{k-2}}$. First note

$$
\begin{aligned}
\lambda_{0}(\lambda) & =\frac{\lambda}{8}\left(\mu+\left(\frac{\mu}{\lambda}\right)^{\frac{1}{k}}\right)\left(4 \mu-\mu^{2}+\left(\frac{\mu}{\lambda}\right)^{\frac{2}{k}}\right), \\
\lambda_{0}^{\prime}(\lambda) & =\frac{1}{8}\left[\left(\mu+\left(\frac{\mu}{\lambda}\right)^{\frac{1}{k}}\right)\left(4 \mu-\mu^{2}+\left(\frac{\mu}{\lambda}\right)^{\frac{2}{k}}\right)-\frac{1}{k}\left(\frac{\mu}{\lambda}\right)^{\frac{1}{k}}\left(4 \mu-\mu^{2}+\left(\frac{\mu}{\lambda}\right)^{\frac{2}{k}}\right)-\frac{2}{k}\left(\frac{\mu}{\lambda}\right)^{\frac{2}{k}}\left(\mu+\left(\frac{\mu}{\lambda}\right)^{\frac{1}{k}}\right)\right] \\
& =\frac{1}{8}\left[\frac{k-1}{k}\left(\frac{\mu}{\lambda}\right)^{\frac{1}{k}}\left(4 \mu-\mu^{2}+\left(\frac{\mu}{\lambda}\right)^{\frac{2}{k}}\right)+\mu\left(4 \mu-\mu^{2}+\left(\frac{\mu}{\lambda}\right)^{\frac{2}{k}}\right)-\frac{2}{k}\left(\frac{\mu}{\lambda}\right)^{\frac{2}{k}}\left(\mu+\left(\frac{\mu}{\lambda}\right)^{\frac{1}{k}}\right)\right] .
\end{aligned}
$$

Because $4 \mu-\mu^{2}+\left(\frac{\mu}{\lambda}\right)^{\frac{2}{k}}-\left(\mu+\left(\frac{\mu}{\lambda}\right)^{\frac{1}{k}}\right)>3 \mu-\mu^{2}-1>0$ and $\mu-\frac{2}{k}\left(\frac{\mu}{\lambda}\right)^{\frac{2}{k}}>\mu-\frac{2}{k}>0, \lambda_{0}(\lambda)$ is an increasing function about $\lambda$ and we have

$$
\lambda_{0} \geqslant \lambda_{0}\left(\frac{\mu}{(2-\mu)^{k}}\right)=\frac{\mu}{(2-\mu)^{k}}>\frac{\mu}{(2-\mu)^{k-2}} .
$$

So (4.34) holds.

(iii). We can see now (2.32) holds for all $x \in\left[1-\frac{1}{\mu}, b_{k}\right]$ by a similar argument as before.

Case (ii). $\lambda \in(2.61,3]$. When $k \geqslant 24$ and $\lambda \in(2.61,3]$, we use the following five cases, Case (1) to Case (5) to prove Lemma 2.1.13.

Firstly, we begin from the following Lemma.

Lemma 4.4.3 For $k \geqslant 10$ and $\lambda \in[2.55,3]$, we have the following inequality,

$$
f_{\mu}\left(f_{\mu}\left(f_{\mu}\left(f_{\mu}\left(f_{\mu}\left(\frac{1}{2}-\frac{1}{2 \sqrt[k]{\mu^{k-1} \lambda}}\right)\right)\right)\right)>\frac{1}{2}-\frac{1}{2 \sqrt[k+3]{\mu^{k+2} \lambda}} .\right.
$$

See Figure 4.14. 


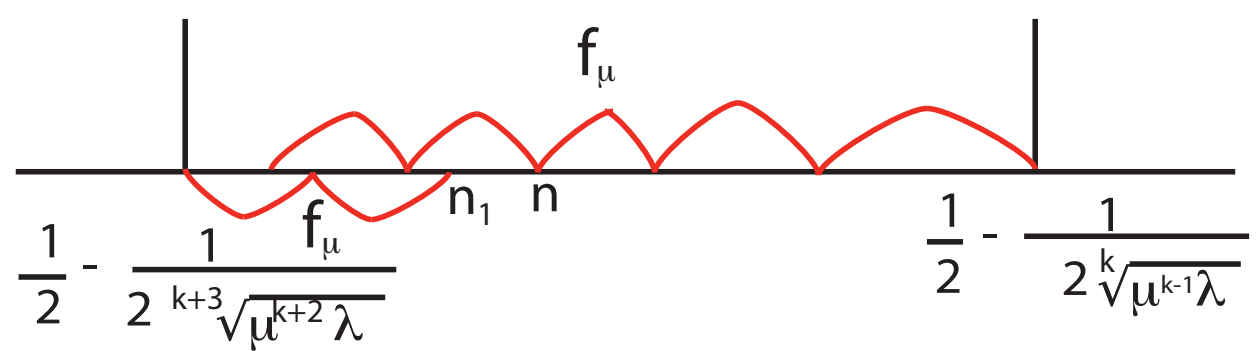

Figure 4.14: $n>n_{1}$

Proof. Firstly, we prove $n>n_{1}$ in order to get (4.37). Here $n=f_{\mu}\left(f_{\mu}\left(f_{\mu}\left(\frac{1}{2}-\frac{1}{2 \sqrt[k]{\mu^{k-1} \lambda}}\right)\right)\right)$ and $f_{\mu}\left(f_{\mu}\left(n_{1}\right)\right)=\frac{1}{2}-\frac{1}{2 \sqrt[k+3]{\mu^{k+2} \lambda}}$. This means we need to prove

$$
\begin{aligned}
& \frac{\left(\mu^{2}-\left(\frac{\mu}{\lambda}\right)^{\frac{2}{k}}\right)\left(4 \mu-\mu^{2}+\left(\frac{\mu}{\lambda}\right)^{\frac{2}{k}}\right)\left[16 \mu-\left(\mu^{2}-\left(\frac{\mu}{\lambda}\right)^{\frac{2}{k}}\right)\left(4 \mu-\mu^{2}+\left(\frac{\mu}{\lambda}\right)^{\frac{2}{k}}\right)\right]}{256 \mu} \\
& >\frac{2 \mu-\sqrt{\mu^{2}-2 \mu+2 \sqrt{\mu^{2}-2 \mu+2\left(\frac{\mu}{\lambda}\right)^{\frac{1}{k+3}}}}}{2 \mu} .
\end{aligned}
$$

For this Let $E(\mu)=\left(\mu^{2}-\left(\frac{\mu}{\lambda}\right)^{\frac{2}{k}}\right)\left(4 \mu-\mu^{2}+\left(\frac{\mu}{\lambda}\right)^{\frac{2}{k}}\right), D(\mu)=\sqrt{\mu^{2}-2 \mu+2 \sqrt{\mu^{2}-2 \mu+2\left(\frac{\mu}{\lambda}\right)^{\frac{1}{k+3}}}}-$ $\mu, B(\mu)=E(16 \mu-E)$. We need to prove $A(\mu, \lambda, k)=B(\mu)+128 D(\mu)>0$. Since $\frac{1.045}{(2-1.045)}>3$, it is easy to see $\mu \in(1,1.045)$.

$$
\begin{aligned}
E^{\prime}(\mu)= & 12 \mu^{2}-4 \mu^{3}+4(\mu-1)\left(\frac{\mu}{\lambda}\right)^{\frac{2}{k}}+\frac{4 \mu}{k}\left(\frac{\mu}{\lambda}\right)^{\frac{2}{k}}-\frac{8}{k}\left(\frac{\mu}{\lambda}\right)^{\frac{2}{k}}-\frac{4}{k \mu}\left(\frac{\mu}{\lambda}\right)^{\frac{4}{k}} \\
> & 12 \mu^{2}-4 \mu^{3}-\frac{8}{k}>0, \\
E(\mu)< & \mu^{2}\left(4 \mu-\mu^{2}+1\right)<8, \\
E^{\prime}(\mu)< & 12 \mu^{2}-4 \mu^{3}+4(\mu-1)<16, \\
E^{\prime \prime}(\mu)= & 24 \mu-12 \mu^{2}+4\left(\frac{\mu}{\lambda}\right)^{\frac{2}{k}}+\frac{8(\mu-1)}{k \mu}\left(\frac{\mu}{\lambda}\right)^{\frac{2}{k}}+\frac{4}{k}\left(\frac{\mu}{\lambda}\right)^{\frac{2}{k}}+\frac{8}{k^{2}}\left(\frac{\mu}{\lambda}\right)^{\frac{2}{k}} \\
& -\frac{16}{k^{2} \mu}\left(\frac{\mu}{\lambda}\right)^{\frac{2}{k}}+\frac{4}{k \mu^{2}}\left(\frac{\mu}{\lambda}\right)^{\frac{4}{k}}-\frac{16}{k^{2} \mu}\left(\frac{\mu}{\lambda}\right)^{\frac{4}{k}} \\
> & \frac{4}{k^{2}}\left(\frac{\mu}{\lambda}\right)^{\frac{2}{k}}\left(k+2-\frac{4}{\mu}\right)+\frac{4}{k^{2} \mu}\left(\frac{\mu}{\lambda}\right)^{\frac{4}{k}}(k-4)>0 .
\end{aligned}
$$

We differentiate $B(\mu)$ with respect to $\mu$ to get

$$
\begin{aligned}
B^{\prime}(\mu) & =E^{\prime}(\mu)(16 \mu-2 E)+16 E, \\
B^{\prime \prime}(\mu) & =E^{\prime \prime}(\mu)(16 \mu-2 E)+2 E^{\prime}(\mu)\left(16-E^{\prime}(\mu)\right)>0 .
\end{aligned}
$$

So $B^{\prime}(\mu)$ is an increasing function with respect to $\mu$. On the other hand, we differentiate 
$D(\mu)$ with respect to $\mu$ to get

$$
\begin{aligned}
& D^{\prime}(\mu)=\frac{\mu-1+\frac{\mu-1+\frac{1}{(k+3) \mu}\left(\frac{\mu}{\lambda}\right)^{\frac{1}{k+3}}}{\sqrt{\mu^{2}-2 \mu+2\left(\frac{\mu}{\lambda}\right)^{\frac{1}{k+3}}}}}{\sqrt{\mu^{2}-2 \mu+2 \sqrt{\mu^{2}-2 \mu+2\left(\frac{\mu}{\lambda}\right)^{\frac{1}{k+3}}}}}-1 \\
& D^{\prime \prime}(\mu)=\frac{1}{\left(\mu^{2}-2 \mu+2 \sqrt{\mu^{2}-2 \mu+2\left(\frac{\mu}{\lambda}\right)^{\frac{1}{k+3}}}\right)^{\frac{3}{2}} \cdot\left(\mu^{2}-2 \mu+2\left(\frac{\mu}{\lambda}\right)^{\frac{1}{k+3}}\right)} \text {. } \\
& {\left[\left(\mu^{2}-2 \mu+2\left(\frac{\mu}{\lambda}\right)^{\frac{1}{k+3}}+\left(1-\frac{k+2}{(k+3)^{2}} \mu^{-\frac{2 k+5}{k+3}} \lambda^{-\frac{1}{k+3}}\right) \sqrt{\mu^{2}-2 \mu+2\left(\frac{\mu}{\lambda}\right)^{\frac{1}{k+3}}}\right.\right.} \\
& \left.-\frac{\left(\mu-1+\frac{1}{k+3} \mu^{-\frac{k+2}{k+3}} \lambda^{-\frac{1}{k+3}}\right)^{2}}{\sqrt{\mu^{2}-2 \mu+2\left(\frac{\mu}{\lambda}\right)^{\frac{1}{k+3}}}}\right) \cdot\left(\mu^{2}-2 \mu+2 \sqrt{\mu^{2}-2 \mu+2\left(\frac{\mu}{\lambda}\right)^{\frac{1}{k+3}}}\right) \\
& \left.-\left((\mu-1) \sqrt{\mu^{2}-2 \mu+2\left(\frac{\mu}{\lambda}\right)^{\frac{1}{k+3}}}+\mu-1+\frac{1}{(k+3) \mu}\left(\frac{\mu}{\lambda}\right)^{\frac{1}{k+3}}\right)^{2}\right] .
\end{aligned}
$$

Let $M_{1}=\left(\mu^{2}-2 \mu+2\left(\frac{\mu}{\lambda}\right)^{\frac{1}{k+3}}\right)\left(\mu^{2}-2 \mu+2 \sqrt{\mu^{2}-2 \mu+2\left(\frac{\mu}{\lambda}\right)^{\frac{1}{k+3}}}\right)-\left((\mu-1) \sqrt{\mu^{2}-2 \mu+2\left(\frac{\mu}{\lambda}\right)^{\frac{1}{k+3}}}\right)^{2}-$

$\left(\mu-1+\frac{1}{(k+3) \mu}\left(\frac{\mu}{\lambda}\right)^{\frac{1}{k+3}}\right)^{2}$ and $M_{2}=\left[\left(1-\frac{k+2}{(k+3)^{2}} \mu^{-\frac{2 k+5}{k+3}} \lambda^{-\frac{1}{k+3}}\right) \sqrt{\mu^{2}-2 \mu+2\left(\frac{\mu}{\lambda}\right)^{\frac{1}{k+3}}}-\frac{\left(\mu-1+\frac{1}{k+3} \mu^{-\frac{k+2}{k+3}} \lambda^{-\frac{1}{k+3}}\right)^{2}}{\sqrt{\mu^{2}-2 \mu+2\left(\frac{\mu}{\lambda}\right)^{\frac{1}{k+3}}}}\right]$.

$\left(\mu^{2}-2 \mu+2 \sqrt{\mu^{2}-2 \mu+2\left(\frac{\mu}{\lambda}\right)^{\frac{1}{k+3}}}\right)-2(\mu-1) \sqrt{\mu^{2}-2 \mu+2\left(\frac{\mu}{\lambda}\right)^{\frac{1}{k+3}}}\left(\mu-1+\frac{1}{(k+3) \mu}\left(\frac{\mu}{\lambda}\right)^{\frac{1}{k+3}}\right)$

and then $D^{\prime \prime}(\mu)=\frac{M_{1}+M_{2}}{\left(\mu^{2}-2 \mu+2 \sqrt{\mu^{2}-2 \mu+2\left(\frac{\mu}{\lambda}\right)^{\frac{1}{k+3}}}\right)^{\frac{3}{2}} \cdot\left(\mu^{2}-2 \mu+2\left(\frac{\mu}{\lambda}\right)^{\frac{1}{k+3}}\right)}$.

Since $k \geqslant 10$ and $\frac{1.1}{(2-1.1)^{10}}>3$, we can easily get $\mu \in(1,1.1)$ and $\left(\frac{\mu}{\lambda}\right)^{\frac{1}{k+3}}>3^{-\frac{1}{13}}>$ 0.9189. Note

(i). $\quad \mu^{2}-2 \mu+2\left(\frac{\mu}{\lambda}\right)^{\frac{1}{k+3}}-\left(\mu-1+\frac{1}{(k+3) \mu}\left(\frac{\mu}{\lambda}\right)^{\frac{1}{k+3}}\right)$
$=(\mu-1)^{2}+2\left(\frac{\mu}{\lambda}\right)^{\frac{1}{k+3}}-\frac{1}{(k+3) \mu}\left(\frac{\mu}{\lambda}\right)^{\frac{1}{k+3}}-\mu>\left(\frac{\mu}{\lambda}\right)^{\frac{1}{k+3}}\left(2-\frac{1}{(k+3) \mu}-\mu\right)>0$,

(ii). $\quad 2 \sqrt{\mu^{2}-2 \mu+2\left(\frac{\mu}{\lambda}\right)^{\frac{1}{k+3}}}-1>2 \sqrt{2\left(\frac{\mu}{\lambda}\right)^{\frac{1}{k+3}}-1}-1>0.83$,

(iii). $\quad \mu-1+\frac{1}{(k+3) \mu}\left(\frac{\mu}{\lambda}\right)^{\frac{1}{k+3}}<1.1-1+\frac{1}{13}<0.83<2 \sqrt{\mu^{2}-2 \mu+2\left(\frac{\mu}{\lambda}\right)^{\frac{1}{k+3}}}-1$.

Hence, $M_{1}>0$.

Second, for $M_{2}$, note

(i). $\quad \frac{2 k^{2}+9 k+10}{(k+3)^{2}}\left(\frac{\mu}{\lambda}\right)^{\frac{1}{k+3}}-1>\frac{2 \cdot 10^{2}+90+10}{13^{2}} \cdot 0.9189-1>0.63$,

(ii). $2(\mu-1)\left(\mu^{2}-2 \mu+2\left(\frac{\mu}{\lambda}\right)^{\frac{1}{k+3}}\right)<2 \cdot 0.1 \cdot\left(\mu^{2}-2 \mu+2 \cdot 0.9189\right)<0.17<0.63$,

(iii). $\quad \mu^{2}-2 \mu+2 \sqrt{\mu^{2}-2 \mu+2\left(\frac{\mu}{\lambda}\right)^{\frac{1}{k+3}}}-\left(\mu-1+\frac{1}{(k+3) \mu}\left(\frac{\mu}{\lambda}\right)^{\frac{1}{k+3}}\right)$ 


$$
\begin{aligned}
& =(\mu-1)^{2}+2 \sqrt{\mu^{2}-2 \mu+2\left(\frac{\mu}{\lambda}\right)^{\frac{1}{k+3}}}-\frac{1}{(k+3) \mu}\left(\frac{\mu}{\lambda}\right)^{\frac{1}{k+3}}-\mu \\
& >2 \sqrt{-1+2 \cdot 0.9189}-\frac{1}{13}-1>0 .
\end{aligned}
$$

Hence, $M_{2}>0$ and then $D^{\prime \prime}(\mu)>0$. Since $\frac{\partial A(\mu, \lambda, k)}{\partial \mu}=B^{\prime}(\mu)+128 D^{\prime}(\mu)$ and $\frac{\partial A(\mu, k)^{2}}{\partial^{2} \mu}=$ $B^{\prime \prime}(\mu)+128 D^{\prime \prime}(\mu)>0, \frac{\partial A(\mu, \lambda, k)}{\partial \mu}$ increases as $\mu$ increases and $\frac{\partial A(\mu, \lambda, k)}{\partial \mu}>\frac{\partial A(1, \lambda, k)}{\partial \mu}$. But

$$
\begin{aligned}
\frac{\partial A(1, \lambda, k)}{\partial \mu}= & E^{\prime}(1)(16-2 E(1))+16 E(1) \\
= & \left(8-\frac{4}{k} \lambda^{-\frac{2}{k}}-\frac{4}{k} \lambda^{-\frac{4}{k}}\right)\left(16-2\left(1-\lambda^{-\frac{2}{k}}\right)\left(3+\lambda^{-\frac{2}{k}}\right)\right)+16\left(1-\lambda^{-\frac{2}{k}}\right)\left(3+\lambda^{-\frac{2}{k}}\right) \\
& +128 \frac{\frac{1}{k+3} \lambda^{-\frac{1}{k+3}}}{\sqrt{-1+2 \sqrt{-1+2 \lambda^{-\frac{1}{k+3}}}} \sqrt{-1+2 \lambda^{-\frac{1}{k+3}}}}-128 \\
= & 8\left(-\frac{5}{k} \lambda^{-\frac{2}{k}}-\frac{7}{k} \lambda^{-\frac{4}{k}}-\frac{3}{k} \lambda^{-\frac{6}{k}}-\frac{1}{k} \lambda^{-\frac{8}{k}}+\frac{16}{k+3} \frac{\lambda^{-\frac{1}{k+3}}}{\sqrt{-1+2 \sqrt{-1+2 \lambda^{-\frac{1}{k+3}}}} \sqrt{-1+2 \lambda^{-\frac{1}{k+3}}}}\right) .
\end{aligned}
$$

Let $F(k)=5 \lambda^{-\frac{2}{k}}+7 \lambda^{-\frac{4}{k}}+3 \lambda^{-\frac{6}{k}}+\lambda^{-\frac{8}{k}}-16 \lambda^{-\frac{3}{k}}$ and differentiate $F(k)$ as to $k$ to get

$$
\begin{aligned}
F^{\prime}(k) & =\frac{2 \ln \lambda}{k^{2}} \lambda^{-\frac{3}{k}}\left(5 \lambda^{\frac{1}{k}}+14 \lambda^{-\frac{1}{k}}+9 \lambda^{-\frac{3}{k}}+4 \lambda^{-\frac{5}{k}}-24\right) \\
& >14 \cdot 3^{-\frac{1}{10}}+9 \cdot 3^{-\frac{3}{10}}+4 \cdot 3^{-\frac{5}{10}}-19>0 .
\end{aligned}
$$

So $F(k)$ increases as $k$ increases and then

$$
F(k)<\lim _{k \rightarrow \infty} F(k)=0 .
$$

This leads to

$$
\frac{\partial A(1, \lambda, k)}{\partial \mu}>128\left(-\frac{1}{k} \lambda^{-\frac{3}{k}}+\frac{1}{k+3} \frac{\lambda^{-\frac{1}{k+3}}}{\sqrt{-1+2 \sqrt{-1+2 \lambda^{-\frac{1}{k+3}}}} \sqrt{-1+2 \lambda^{-\frac{1}{k+3}}}}\right) .
$$

Let $H(k)=\frac{k^{2}}{(k+3)^{2}} \lambda^{\frac{2(2 k+9)}{k(k+3)}}-\left(-1+2 \sqrt{-1+2 \lambda^{-\frac{1}{k+3}}}\right)\left(-1+2 \lambda^{-\frac{1}{k+3}}\right)$. Then

$$
H^{\prime}(k)=\frac{2 \lambda^{-\frac{1}{k+3}}}{(k+3)^{2}} H_{1}(k),
$$

where $H_{1}(k)=\lambda^{\frac{5 k+18}{k(k+3)}}\left(\frac{3 k}{k+3}-3 \ln \lambda+\frac{k^{2}}{(k+3)^{2}} \ln \lambda\right)-\left(3 \sqrt{-1+2 \lambda^{-\frac{1}{k+3}}}-1\right) \ln \lambda$. Then differentiate $H_{1}(k)$ as to $k$ to get

$$
H_{1}^{\prime}(k)=\frac{\lambda^{\frac{5 k+18}{k(k+3)}} \ln \lambda}{(k+3)^{2}}\left[\frac{-5 k^{2}-36 k-54}{k^{2}}\left(\frac{3 k}{k+3}-3 \ln \lambda+\frac{k^{2}}{(k+3)^{2}} \ln \lambda\right)+\frac{9}{\ln \lambda}+\frac{k}{k+3}\right.
$$




$$
\left.+\frac{5 k}{k+3}-\frac{3 \lambda^{-\frac{6}{k}} \ln \lambda}{\sqrt{-1+2 \lambda^{-\frac{1}{k+3}}}}\right] .
$$

Let $a=\ln \lambda$, then $a \in(0.936,1.1)$ for $\lambda \in[2.55,3]$. For $k \geqslant 10, \frac{-5 k^{2}-36 k-54}{k^{2}} \in[-9.14,-5)$. Since $\left(\frac{-5 k^{2}-36 k-54}{k^{2}}\left(\frac{3 k}{k+3}-3 a+\frac{k^{2}}{(k+3)^{2}} a\right)+\frac{9}{a}\right)_{a}^{\prime}=\frac{-5 k^{2}-36 k-54}{k^{2}}\left(-3+\frac{k^{2}}{(k+3)^{2}}\right)-\frac{9}{a^{2}}>(-5) \cdot(-2)-$ $\frac{9}{0.936}>0$, then $\frac{-5 k^{2}-36 k-54}{k^{2}}\left(\frac{3 k}{k+3}-3 \ln \lambda+\frac{k^{2}}{(k+3)^{2}} \ln \lambda\right)+\frac{9}{\ln \lambda}+\frac{k}{k+3}>-9.14(3-2 \cdot \ln 2.55)+$ $\frac{9}{\ln 2.55}+\frac{10}{13}>0$. On the other hand, $\left(2 \lambda^{-\frac{1}{k+3}}-1-2 \lambda^{-\frac{12}{k}}\right)_{k}^{\prime}=\frac{2 \lambda^{-\frac{12}{k}} \ln \lambda}{(k+3)^{2}}\left(\lambda^{\frac{11 k+36}{k(k+3)}}-6 \frac{(k+3)^{2}}{k^{2}}\right)<$ $\frac{2 \lambda^{-\frac{12}{k}} \ln \lambda}{(k+3)^{2}}\left(3^{\frac{110+36}{130}}-6\right)<0$, so we have $\frac{\lambda^{-\frac{6}{k}}}{\sqrt{-1+2 \lambda^{-\frac{1}{k+3}}}}<1$ and $\frac{5 k}{k+3}-\frac{3 \lambda^{-\frac{6}{k}} \ln \lambda}{\sqrt{-1+2 \lambda^{-} \frac{1}{k+3}}}>\frac{50}{13}-3.3>$ 0 . Thus it is obvious that $H_{1}^{\prime}(k)>0$ and we can get

$$
H_{1}(k)<\lim _{k \rightarrow \infty} H_{1}(k)=1-2 \ln \lambda<0 .
$$

So $H^{\prime}(k)<0$ and $H(k)$ decrease as $k$ increases. Then we can get $H(k)>\lim _{k \rightarrow \infty} H(k)>0$ and $\frac{\partial A(\mu, \lambda, k)}{\partial \mu}>\frac{\partial A(1, \lambda, k)}{\partial \mu}>0$. As a result, $A(\mu)$ is an increasing function as to $\mu$. So

$$
\begin{aligned}
A(\mu, \lambda, k)> & A(1, \lambda, k)=\left(3-2 \lambda^{-\frac{2}{k}}-\lambda^{-\frac{4}{k}}\right)\left(13+2 \lambda^{-\frac{2}{k}}+\lambda^{-\frac{4}{k}}\right) \\
& +128 \sqrt{-1+2 \sqrt{-1+2 \lambda^{-\frac{1}{k+3}}}}-128 .
\end{aligned}
$$

But

$$
\begin{aligned}
\frac{\partial A(1, \lambda, k)}{\partial \lambda}= & \frac{8}{\lambda}\left(\frac{5 \lambda^{-\frac{2}{k}}}{k}+\frac{7 \lambda^{-\frac{4}{k}}}{k}+\frac{3 \lambda^{-\frac{6}{k}}}{k}+\frac{\lambda^{-\frac{8}{k}}}{k}\right. \\
& \left.+\frac{16 \lambda^{-\frac{1}{k+3}}}{(k+3) \sqrt{-1+2 \sqrt{-1+2 \lambda^{-\frac{1}{k+3}}}} \cdot \sqrt{-1+2 \lambda^{-\frac{1}{k+3}}}}\right) \\
= & \frac{1}{\lambda}\left(-\frac{A(1, k, \lambda)}{\partial \mu}\right)<0 .
\end{aligned}
$$

So $A(1, \lambda, k)$ is a decreasing function as to $\lambda$ and $A(1, \lambda, k)>A(1,3, k)$.

Let $G(k)=5 \lambda^{-\frac{2}{k}}+7 \lambda^{-\frac{4}{k}}+3 \lambda^{-\frac{6}{k}}+\lambda^{-\frac{8}{k}}-16 \lambda^{-\frac{4}{k}}$, then we differentiate $G$ with respect to $k$ for $k \geqslant 10$,

$$
\begin{aligned}
& G^{\prime}(k)=\frac{2 \lambda^{-\frac{4}{k}}}{k^{2}}\left(5 \lambda^{\frac{2}{k}}+9 \lambda^{-\frac{2}{k}}+4 \lambda^{-\frac{4}{k}}-18\right), \\
& \left(5 \lambda^{\frac{2}{k}}+9 \lambda^{-\frac{2}{k}}+4 \lambda^{-\frac{4}{k}}\right)_{k}^{\prime}=\frac{2 \lambda^{-\frac{2}{k}} \ln \lambda}{k^{2}}\left(-5 \lambda^{\frac{4}{k}}+9+8 \lambda^{-\frac{4}{k}}\right)>0 .
\end{aligned}
$$

So we can get

$$
5 \lambda^{\frac{2}{k}}+9 \lambda^{-\frac{2}{k}}+4 \lambda^{-\frac{4}{k}}-18<0 .
$$

So $G(k)$ is a decreasing function as to $k$ and $G(k)>\lim _{k \rightarrow \infty} G(k)=0$. THen we can get

$$
\frac{\partial A(1, \lambda, k)}{\partial k}=8 \ln \lambda\left(-\frac{5 \lambda^{-\frac{2}{k}}+7 \lambda^{-\frac{4}{k}}+3 \lambda^{-\frac{6}{k}}+\lambda^{-\frac{8}{k}}}{k^{2}}\right.
$$




$$
\begin{gathered}
\left.+\frac{16 \lambda^{-\frac{1}{k+3}}}{(k+3)^{2} \sqrt{-1+2 \sqrt{-1+2 \lambda^{-\frac{1}{k+3}}}} \cdot \sqrt{-1+2 \lambda^{-\frac{1}{k+3}}}}\right) \\
<\ln \lambda\left(-\frac{16 \lambda^{-\frac{4}{k}}}{k^{2}}+\frac{16 \lambda^{-\frac{1}{k+3}}}{(k+3)^{2} \sqrt{-1+2 \sqrt{-1+2 \lambda^{-\frac{1}{k+3}}}} \cdot \sqrt{-1+2 \lambda^{-\frac{1}{k+3}}}}\right) .
\end{gathered}
$$

Let $P(k, \lambda)=\frac{k^{4}}{(k+3)^{4}} \lambda^{\frac{6 k+24}{k(k+3)}}-\left(-1+2 \sqrt{-1+2 \lambda^{-\frac{1}{k+3}}}\right)\left(-1+2 \lambda^{-\frac{1}{k+3}}\right)$, then we differentiate $P(k, \lambda)$ about $k$ to get

$$
\begin{aligned}
\frac{\partial P(k, \lambda)}{\partial \lambda}= & \frac{2 \lambda^{-1-\frac{1}{k+3}}}{k+3}\left[\frac{k^{3}(3 k+12)}{(k+3)^{4}} \lambda^{\frac{7 k+24}{k(k+3)}}+3 \sqrt{-1+2 \lambda^{-\frac{1}{k+3}}}-1\right]>0 \\
\frac{\partial P(k, \lambda)}{\partial k}= & \frac{2 \lambda^{-\frac{1}{k+3}}}{(k+3)^{2}}\left[\lambda^{\frac{7 k+24}{k(k+3)}} \frac{3 k^{2}}{(k+3)^{2}}\left(\frac{2 k}{k+3}-\frac{k^{2}+8 k+12}{(k+3)^{2}} \ln \lambda\right)-\ln \lambda\left(3 \sqrt{-1+2 \lambda^{-\frac{1}{k+3}}}-1\right)\right] \\
:= & \frac{2 \lambda^{-\frac{1}{k+3}}}{(k+3)^{2}} Q(k, \lambda), \\
\frac{\partial Q(k, \lambda)}{\partial k=}= & \frac{3 \lambda^{\frac{7 k+24}{k(k+3)}}}{(k+3)^{2}}\left(\frac{2 k}{k+3}-\frac{k^{2}+8 k+12}{(k+3)^{2}} \ln \lambda\right)\left(\frac{6 k}{k+3}-\frac{7 k^{2}+48 k+72}{(k+3)^{2}} \ln \lambda\right) \\
& +\lambda^{\frac{7 k+24}{k(k+3)}} \frac{3 k^{2}}{(k+3)^{4}}\left(6-\frac{2 k}{k+3} \ln \lambda\right)-\frac{3 \lambda^{-\frac{1}{k+3}(\ln \lambda)^{2}}}{(k+3)^{2} \sqrt{-1+2 \lambda^{-\frac{1}{k+3}}}} \\
> & \frac{3 \lambda^{\frac{7 k+24}{k(k+3)}}}{(k+3)^{2}} \cdot \frac{1.064 k^{2}-1.488 k-11.232}{(k+3)^{2}} \cdot \frac{-1.7 k^{2}-34.8 k-79.2}{(k+3)^{2}} \\
& +\lambda^{\frac{7 k+24}{k(k+3)}} \frac{3 k^{2}}{(k+3)^{4}} \cdot \frac{3.8 k+18}{k+3}-\frac{3 \lambda^{-\frac{1}{k+3}}(\ln \lambda)^{2}}{(k+3)^{2} \sqrt{-1+2 \lambda^{-\frac{1}{k+3}}} .}
\end{aligned}
$$

So $P(k, \lambda)$ increases as $\lambda$ increases and $P(k, \lambda) \leqslant P(k, 3)$. Since it is obvious that $-1+$ $2 \lambda^{-\frac{1}{k+3}}>0.8379$ for $k \geqslant 10$, so

$$
\begin{aligned}
\left(\lambda^{\frac{8}{k}} \frac{k^{2}}{(k+3)^{2}} \sqrt{-1+2 \lambda^{-\frac{1}{k+3}}}\right)_{k}^{\prime}= & \frac{\lambda^{\frac{8}{k}}}{(k+3)^{2} \sqrt{-1+2 \lambda^{-\frac{1}{k+3}}}}\left[\left(-8 \ln \lambda+\frac{6 k}{k+3}\right)\left(-1+2 \lambda^{-\frac{1}{k+3}}\right)\right. \\
& +\frac{k^{2}}{(k+3)^{2}} \lambda^{\left.-\frac{1}{k+3} \ln \lambda\right]} \\
< & \frac{\lambda^{\frac{8}{k}}}{(k+3)^{3} \sqrt{-1+2 \lambda^{-\frac{1}{k+3}}}}(0.8379(-1.488 k-22.464)(k+3)+k)<0 .
\end{aligned}
$$

So $\lambda^{\frac{8}{k}} \frac{k^{2}}{(k+3)^{2}} \sqrt{-1+2 \lambda^{-\frac{1}{k+3}}}>1$. Thus we have

$\frac{\partial Q(k, \lambda)}{\partial k}>\frac{3 \lambda^{\frac{7 k+24}{k(k+3)}}}{(k+3)^{6}}\left[\left(1.064 k^{2}-1.488 k-11.232\right)\left(-1.7 k^{2}-34.8 k-79.2\right)+k^{2}(k+3)(2.59 k+14.37)\right]$ 


$$
\begin{aligned}
+ & \frac{3 \lambda^{-\frac{1}{k+3}}}{(k+3)^{2}}\left[\lambda^{\frac{8}{k}} \frac{k^{2}}{(k+3)^{2}} \sqrt{-1+2 \lambda^{-\frac{1}{k+3}}} 1.21-(\ln \lambda)^{2}\right] \\
> & \frac{3 \lambda^{\frac{7 k+24}{k(k+3)}}}{(k+3)^{6}}\left(0.78 k^{4}-12.36 k^{3}+29.7 k^{2}+508.7 k+889.5\right)>0 .
\end{aligned}
$$

So $Q(k, \lambda)$ increases as $k$ increases. Because $\lim _{k \rightarrow \infty} Q(k, \lambda)=6-5 \ln \lambda>0$ and $Q(10, \lambda)=\lambda^{\frac{94}{130}} \cdot \frac{300}{169}\left(\frac{20}{13}-\frac{192}{169} \ln \lambda\right)-\ln \lambda\left(3 \sqrt{-1+2 \lambda^{-\frac{1}{13}}}-1\right)$. It is easy to see that

$$
\frac{\partial Q(10, \lambda)}{\partial \lambda}=\frac{300}{169} \lambda^{-1+\frac{94}{130}}\left(\frac{94}{130}\left(\frac{30}{13}-\frac{192}{169} \ln \lambda\right)-\frac{192}{169}\right)-\frac{1}{\lambda}\left(3 \sqrt{-1+2 \lambda^{-\frac{1}{13}}}-1-\frac{\lambda^{-\frac{1}{13}} \ln \lambda}{13 \sqrt{-1+2 \lambda^{-\frac{1}{13}}}}\right)<0 .
$$

So $Q(10, \lambda)<Q(10,2.55)<0$. Note that there exists $k_{0}$ such that $Q(k, \lambda)<0$ for $k \in\left[10, k_{0}\right]$ and $Q(k, \lambda)>0$ for $k>k_{0}$, i.e. $P(k, \lambda)$ decreases first and then increases as $k$ increases. Since $\lim _{k \rightarrow \infty} P(k, \lambda)=0$, and $P(13,3)<0$, we can get $P(k, \lambda)<0$ for $k \geqslant 13$ and so $A(1, k)$ decrease as $k$ increases. Therefore,

$$
A(\mu, \lambda, k)>\lim _{k \rightarrow \infty} A(1, \lambda, k)=0 .
$$

While for $k \in[10,12]$, recalling that $A(\mu, \lambda, k)>A(1,3, k)$ and $A(1,3, k)>0$ when $k=10,11,12$. So $A(\mu, \lambda, k)>0$ for all $k \geqslant 10$. Lemma 4.4 .3 is proved.

Now, we prove (4.22) still holds for $\lambda \in(2.61,3]$ when $k \geqslant 24$. We mainly use Lemma 4.4.3 . Since $\frac{1.044}{(2-1.044)^{24}}>3$, it is easy to get $\mu \in(1,1.044)$. When $k \geqslant 24$, we divide $k$ into cases $k=5 m, 5 m+1,5 m+2,5 m+3$ and $5 m+4$.

Case (1). $k=5 m, m \geqslant 5$, see Figure 4.15 , we prove

$$
f_{\lambda}\left(\frac{1}{2}-\frac{1}{2 \sqrt[5 m]{\mu^{5 m-1} \lambda}}\right)>\frac{1}{2}-\frac{1}{2 \sqrt[2 m]{\mu^{2 m-1} \lambda}}
$$

Let $A(m)=\frac{\lambda}{4}-\frac{1}{4 \mu}\left(\frac{\lambda}{\mu}\right)^{\frac{5 m-2}{5 m}}-\frac{1}{2}+\frac{1}{2 \mu}\left(\frac{\mu}{\lambda}\right)^{\frac{1}{2 m}}$ and differentiate $A(m)$ with respect to $m$ to

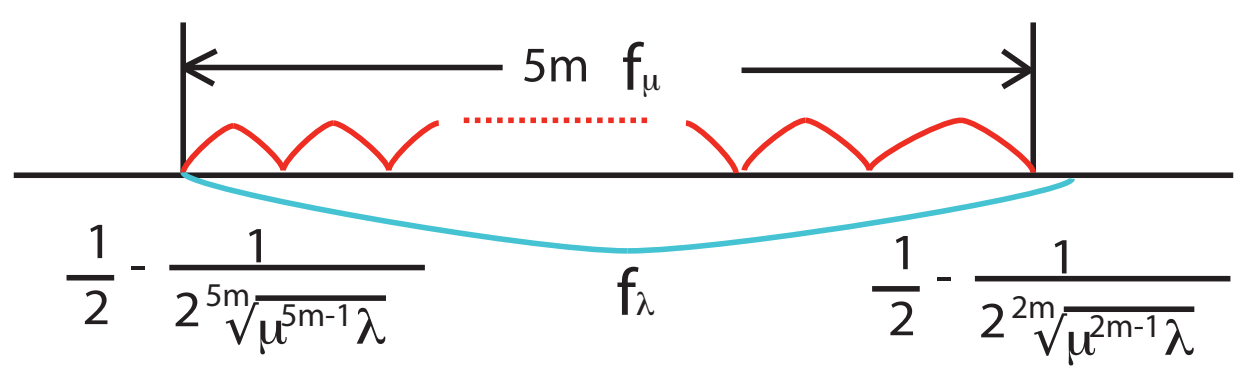

Figure 4.15: $f_{\lambda}\left(\frac{1}{2}-\frac{1}{2 \sqrt[5 m]{\mu^{5 m-1} \lambda}}\right)>\frac{1}{2}-\frac{1}{2 \sqrt[2 m]{\mu^{2 m-1} \lambda}}$

get

$$
\frac{\partial A(m)}{\partial m}=\frac{\left(\frac{\mu}{\lambda}\right)^{\frac{1}{2 m}}}{2 \mu m^{2}} \ln \left(\frac{\lambda}{\mu}\right)\left(-\frac{2}{5}\left(\frac{\lambda}{\mu}\right)^{\frac{10 m+1}{10 m}}+1\right)<\frac{\left(\frac{\mu}{\lambda}\right)^{\frac{1}{2 m}}}{2 \mu m^{2}} \ln \left(\frac{\lambda}{\mu}\right)\left(\frac{2 \cdot 2.61}{5 \cdot 1.044}-1\right)>0 .
$$


That is to say $A(m)$ decreases as $k$ increases and $A(m)>\lim _{m \rightarrow \infty} A(m)=0$. Then (4.39) is proved. By $(4.25), f_{\lambda} \underbrace{f_{\mu} \cdots f_{\mu}}_{k}(x)-x$ reaches the minimum value at $x=1-\frac{1}{\mu}$ and $x=y *$ for $x \in\left[1-\frac{1}{\mu}, y^{\star}\right]$. From (4.39) and $a>y^{\star}$, where $f_{\mu} \underbrace{f_{\mu} \cdots f_{\mu}}_{k-1}(a)=\frac{1}{2}-\frac{1}{2 \sqrt[5 m]{\mu^{5 m-1} \lambda}}$, we know (4.34) holds. Considering that $f_{\lambda} \underbrace{f_{\mu} \cdots f_{\mu}}_{k}\left(1-\frac{1}{\mu}\right)>1-\frac{1}{\mu}$, we can easily see that (2.32) holds.

Case (2). $k=5 m+1, m \geqslant 5$. See Figure 4.16, by Lemma 4.4.3, we just need to prove

$$
f_{\lambda}\left(f_{\mu}\left(\frac{1}{2}-\frac{1}{2 \sqrt[5 m+1]{\mu^{5 m} \lambda}}\right)\right)>\frac{1}{2}-\frac{1}{2 \sqrt[2 m+1]{\mu^{2 m} \lambda}} .
$$

Let $A(\mu, m)=\left(\mu^{2}-\left(\frac{\mu}{\lambda}\right)^{\frac{2}{5 m+1}}\right)\left(4 \mu-\mu^{2}+\left(\frac{\mu}{\lambda}\right)^{\frac{2}{5 m+1}}\right)-\frac{8 \mu^{2}}{\lambda}+8\left(\frac{\mu}{\lambda}\right)^{\frac{2 m+2}{2 m+1}}$ and we differentiate

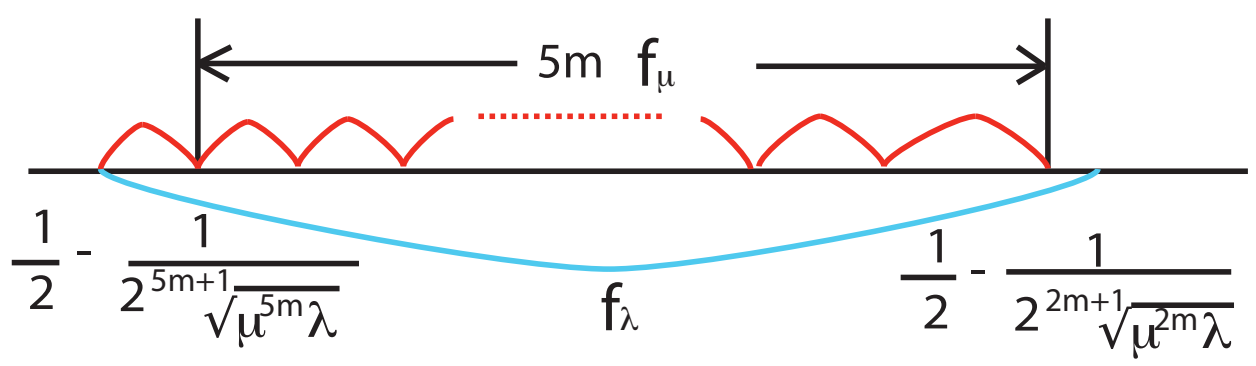

Figure 4.16: $f_{\lambda}\left(f_{\mu}\left(\frac{1}{2}-\frac{1}{2 \sqrt[5 m+1]{\mu^{5 m} \lambda}}\right)\right)>\frac{1}{2}-\frac{1}{2 \sqrt[2 m+1]{\mu^{2 m} \lambda}}$

$A(\mu, m)$ with respect to $\mu$.

$$
\begin{aligned}
\frac{1}{2} \frac{\partial A(\mu, m)}{\partial \mu}= & 6 \mu^{2}-2 \mu^{3}-\frac{8 \mu}{\lambda}+(2 \mu-2)\left(\frac{\mu}{\lambda}\right)^{\frac{2}{5 m+1}}+\frac{2 \mu}{5 m+1}\left(\frac{\mu}{\lambda}\right)^{\frac{2}{5 m+1}}-\frac{2}{(5 m+1) \mu}\left(\frac{\mu}{\lambda}\right)^{\frac{4}{5 m+1}} \\
& -\frac{4}{5 m+1}\left(\frac{\mu}{\lambda}\right)^{\frac{2}{5 m+1}}+4 \frac{2 m+2}{(2 m+1) \lambda}\left(\frac{\mu}{\lambda}\right)^{\frac{1}{2 m+1}}
\end{aligned}
$$

Since $6 \mu^{2}-2 \mu^{3}-\frac{8 \mu}{\lambda}>2 \mu\left(3 \mu-\mu^{2}-1\right)>0,\left(\frac{\lambda}{\mu}\right)^{\frac{m-1}{(2 m+1)(5 m+1)}}<1.02$ and $\frac{\left(\frac{\mu}{\lambda}\right)^{\frac{1}{2 m+1}}}{\lambda}-\frac{\left(\frac{\mu}{\lambda}\right)^{\frac{2}{5 m+1}}}{5 m+1}=$ $\frac{\left(\frac{\mu}{\lambda}\right)^{\frac{1}{2 m+1}}}{\lambda}\left(1-\frac{\lambda\left(\frac{\lambda}{\mu}\right)^{\frac{m-1}{(2 m+1)(5 m+1)}}}{5 m+1}\right)>0$, we can easily get $\frac{\partial A(\mu, m)}{\partial \mu}>0$ and $A(\mu, m)$ increases as $\mu$ increases. Thus

$$
A(\mu, m)>A(1, m)=3-2 \lambda^{-\frac{2}{5 m+1}}-\lambda^{-\frac{4}{5 m+1}}-\frac{8}{\lambda}+8 \lambda^{-\frac{2 m+2}{2 m+1}} .
$$

Note $\frac{24(5 m+1)^{3}}{5(2 m+1)^{3}}>63.38$ for $m \geqslant 5$, we have

$$
\begin{aligned}
\frac{\partial A(1, m)}{\partial m} & =\frac{-5 \lambda^{-\frac{2 m+2}{2 m+1}} \ln \lambda}{(5 m+1)^{2}}\left[\lambda^{\frac{10 m^{2}+8 m}{(5 m+1)(2 m+1)}}\left(1+\lambda^{-\frac{2}{5 m+1}}\right)-\frac{4(5 m+1)^{2}}{5(2 m+1)^{2}}\right]=: \frac{-5 \lambda^{-\frac{2 m+2}{2 m+1}} \ln \lambda}{(5 m+1)^{2}} B(m), \\
B^{\prime}(m) & =\lambda^{\frac{10 m^{2}+8 m}{(5 m+1)(2 m+1)}} \frac{-10 m^{2}+20 m+8}{(2 m+1)^{2}(5 m+1)^{2}} \ln \lambda\left(1+\lambda^{-\frac{2}{5 m+1}}\right)
\end{aligned}
$$




$$
\begin{aligned}
& +\lambda^{\frac{10 m^{2}+4 m-2}{(5 m+1)(2 m+1)}} \frac{10}{(5 m+1)^{2}} \ln \lambda-\frac{24(5 m+1)}{5(2 m+1)^{3}} \\
< & \frac{1}{(5 m+1)^{2}}\left[10 \lambda \ln \lambda-\frac{24(5 m+1)^{3}}{5(2 m+1)^{3}}\right]<\frac{1}{(5 m+1)^{2}}(30 \ln 3-63.38)<0 .
\end{aligned}
$$

So $B(m)$ decreases as $m$ increases and $B(m)>\lim _{m \rightarrow \infty} B(m)=2 \lambda-5>0$ as $\lambda \in(2.61,3]$. Therefore, $\frac{\partial A(1, m)}{\partial m}<0$ and

$$
A(\mu, m)>\lim _{m \rightarrow \infty} A(1, m)=0 .
$$

So (4.34) holds. By (4.25), $f_{\lambda} \underbrace{f_{\mu} \cdots f_{\mu}}_{k}(x)-x$ attains the minimum value at $x=1-\frac{1}{\mu}$ and $x=y *$, then because of $(4.34)$ and $f_{\lambda} \underbrace{f_{\mu} \cdots f_{\mu}}_{k}\left(1-\frac{1}{\mu}\right)>1-\frac{1}{\mu},(2.32)$ holds.

Case (3). $k=5 m+2, m \geqslant 5$. See Figure 4.17, we will prove the following inequality,

$$
f_{\lambda}\left(f_{\mu}\left(\frac{1}{2}-\frac{1}{2 \sqrt[5 m+2]{\mu^{5 m+1} \lambda}}\right)\right)>\frac{1}{2}-\frac{\sqrt{\mu^{2}-2 \mu+2\left(\frac{\mu}{\lambda}\right)^{\frac{1}{2 m+2}}}}{2 \mu} .
$$

Let $A(\mu, m)=\left(\mu^{2}-\left(\frac{\mu}{\lambda}\right)^{\frac{2}{5 m+2}}\right)\left(4 \mu-\mu^{2}+\left(\frac{\mu}{\lambda}\right)^{\frac{2}{5 m+2}}\right)-\frac{8 \mu^{2}}{\lambda}+\frac{8 \mu}{\lambda} \sqrt{\mu^{2}-2 \mu+2\left(\frac{\mu}{\lambda}\right)^{\frac{1}{2 m+2}}}$ and

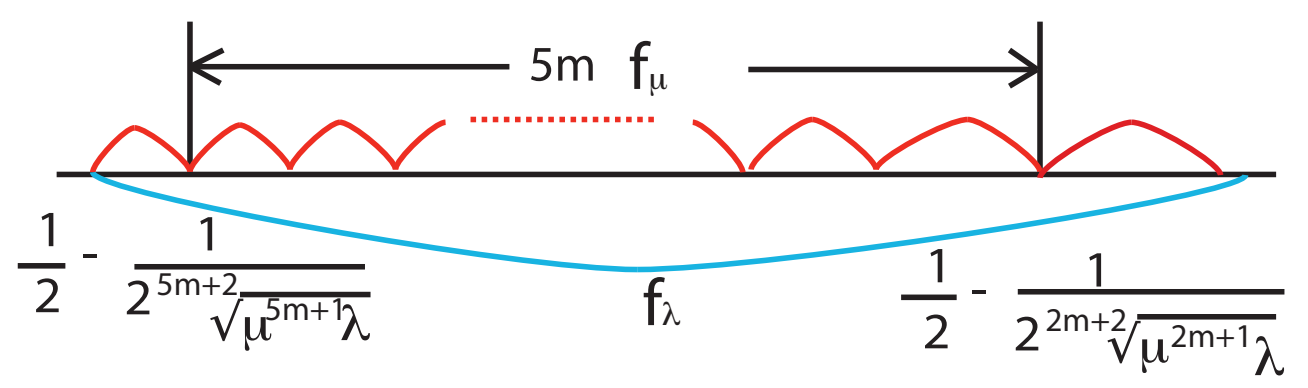

Figure 4.17: $f_{\lambda}\left(f_{\mu}\left(\frac{1}{2}-\frac{1}{2 \sqrt[5 m+2]{\mu^{5 m+1} \lambda}}\right)\right)>\frac{1}{2}-\frac{\sqrt{\mu^{2}-2 \mu+2\left(\frac{\mu}{\lambda}\right)^{\frac{1}{2 m+2}}}}{2 \mu}$ differentiate $A(\mu, m)$ as to $\mu$ to get

$$
\begin{aligned}
\frac{\partial A(\mu, m)}{2 \partial \mu}= & 6 \mu^{2}-2 \mu^{3}-\frac{8 \mu}{\lambda}+(2 \mu-2)\left(\frac{\mu}{\lambda}\right)^{\frac{2}{5 m+2}}+\frac{2 \mu}{5 m+2}\left(\frac{\mu}{\lambda}\right)^{\frac{2}{5 m+2}}-\frac{2}{(5 m+2) \mu}\left(\frac{\mu}{\lambda}\right)^{\frac{4}{5 m+2}} \\
& -\frac{4}{5 m+2}\left(\frac{\mu}{\lambda}\right)^{\frac{2}{5 m+2}}+\frac{4}{\lambda} \sqrt{\mu^{2}-2 \mu+2\left(\frac{\mu}{\lambda}\right)^{\frac{1}{2 m+2}}}+\frac{4 \mu}{\lambda} \cdot \frac{\mu-1+\frac{1}{(2 m+2) \mu}\left(\frac{\mu}{\lambda}\right)^{\frac{1}{2 m+2}}}{\sqrt{\mu^{2}-2 \mu+2\left(\frac{\mu}{\lambda}\right)^{\frac{1}{2 m+2}}}} .
\end{aligned}
$$

For $m \geqslant 5,\left(\frac{\mu}{\lambda}\right)^{\frac{1}{2 m+2}}>0.91, \sqrt{\mu^{2}-2 \mu+2\left(\frac{\mu}{\lambda}\right)^{\frac{1}{2 m+2}}}>0.9$ and $\frac{\sqrt{\mu^{2}-2 \mu+2\left(\frac{\mu}{\lambda}\right)^{\frac{1}{2 m+2}}}}{\lambda}>0.3>$ $\frac{1}{5 m+2}$. So $\frac{\partial A(\mu, m)}{\partial \mu}>0, A(\mu, m)>A(1, m)$ and

$$
A(1, m)=3-2 \lambda^{-\frac{2}{5 m+2}}-\lambda^{-\frac{4}{5 m+2}}-\frac{8}{\lambda}+\frac{8 \sqrt{-1+2 \lambda^{-\frac{1}{2 m+2}}}}{\lambda},
$$




$$
\begin{aligned}
\frac{\partial A(1, m)}{\partial m}= & \frac{-4 \lambda^{-1-\frac{1}{2 m+2}} \ln \lambda}{(5 m+2)^{2} \sqrt{-1+2 \lambda^{-\frac{1}{2 m+2}}}} \cdot\left[\lambda^{\frac{10 m^{2}+15 m+2}{(5 m+2)(2 m+2)}}\left(1+\lambda^{-\frac{2}{5 m+2}}\right) \sqrt{-1+2 \lambda^{-\frac{1}{2 m+2}}}-\frac{4(5 m+2)^{2}}{5(2 m+2)^{2}}\right] \\
:= & \frac{-4 \lambda^{-1-\frac{1}{2 m+2}} \ln \lambda}{(5 m+2)^{2} \sqrt{-1+2 \lambda^{-\frac{1}{2 m+2}}}} B(m) \\
B^{\prime}(m)= & \frac{\lambda^{\frac{10 m^{2}+15 m+2}{(5 m+2)(2 m+2)}} \ln \lambda}{(5 m+2)^{2}} \sqrt{-1+2 \lambda^{-\frac{1}{2 m+2}}}\left[\frac{-10 m^{2}+2}{(2 m+2)^{2}}\left(1+\lambda^{-\frac{2}{5 m+2}}\right)+2 \lambda^{-\frac{2}{5 m+2}}\right]-\frac{48(5 m+2)}{5(2 m+2)^{3}} \\
& +\frac{\ln \lambda}{(5 m+2)^{2}}\left[(10-2) \lambda^{\frac{10 m^{2}+11 m-2}{(5 m+2)(2 m+2)}} \sqrt{-1+2 \lambda^{-\frac{1}{2 m+2}}}\right. \\
& \left.+\lambda^{\frac{5 m}{5 m+2}} \frac{1+\lambda^{-\frac{2}{5 m+2}}}{\sqrt{-1+2 \lambda^{-\frac{1}{2 m+2}}}} \frac{2(5 m+2)^{2}}{(2 m+2)^{2}}\right]
\end{aligned}
$$

For $m \geqslant 5, \frac{48(5 m+2)^{3}}{5(2 m+2)^{3}}>109.35, \frac{(5 m+2)^{2}}{(2 m+2)^{2}}<\frac{25}{4}, \lambda^{\frac{10 m^{2}+11 m-2}{(5 m+2)(2 m+2)}}<2.794, \lambda^{-\frac{1}{2 m+2}}>0.91$ and we have

$$
\begin{aligned}
&\left(\frac{\lambda^{\frac{5 m}{5 m+2}}+\lambda^{\frac{5 m-2}{5 m+2}}}{\left.\sqrt{-1+2 \lambda^{-\frac{1}{2 m+2}}}\right)_{m}^{\prime}=} \frac{10 \ln \lambda}{(5 m+2)^{2}\left(-1+2 \lambda^{\left.-\frac{1}{2 m+2}\right)^{\frac{3}{2}}}\left[\left(-1+2 \lambda^{-\frac{1}{2 m+2}}\right)\left(\lambda^{\frac{5 m}{5 m+2}}+2 \lambda^{\frac{5 m-2}{5 m+2}}\right)\right.\right.}\right. \\
&\left.-\left(\lambda^{\frac{5 m}{5 m+2}}+\lambda^{\frac{5 m-2}{5 m+2}}\right) \frac{(5 m+2)^{2}}{5(2 m+2)^{2}}\right] \\
&> \frac{10 \ln \lambda}{(5 m+2)^{2}\left(-1+2 \lambda^{-\frac{1}{2 m+2}}\right)^{\frac{3}{2}}}\left[\left(\lambda^{\frac{5 m}{5 m+2}}+\lambda^{\frac{5 m-2}{5 m+2}}\right)\left(-1+0.75 \lambda^{-\frac{1}{2 m+2}}\right)\right. \\
&\left.+\lambda^{\frac{5 m-2}{5 m+2}}\left(-1+2 \lambda^{-\frac{1}{2 m+2}}\right)\right] .
\end{aligned}
$$

Since $-1+2 \lambda^{-\frac{1}{2 m+2}}+\frac{7}{3}\left(-1+0.75 \lambda^{-\frac{1}{2 m+2}}\right)=\frac{15}{4} \lambda^{-\frac{1}{2 m+2}}-\frac{10}{3}>0$ and $\frac{7}{3} \lambda^{\frac{5 m-2}{5 m+2}}-\left(\lambda^{\frac{5 m}{5 m+2}}+\right.$ $\left.\lambda^{\frac{5 m-2}{5 m+2}}\right)=\frac{4}{3}\left(\lambda^{\frac{5 m-2}{5 m+2}}-0.75 \lambda^{\frac{5 m}{5 m+2}}\right)>0$, we can get $\left(\frac{\lambda^{\frac{5 m}{5 m+2}}+\lambda^{5 m+2}}{\sqrt{-1+2 \lambda^{-\frac{1}{2 m+2}}}}\right)_{m}^{\prime}>0, \frac{\lambda^{5 m m+2}+\lambda^{5 m-2}}{\sqrt{-1+2 \lambda^{-\frac{1}{2 m+2}}}}<$ $2 \lambda<6$ and

$$
B^{\prime}(m)<\frac{1}{(5 m+2)^{2}}(-109.35+1.1 \cdot(8 \cdot 2.794+6 \cdot 12.5))<0 .
$$

Therefore, $B(m)$ decrease as $m$ increases and $B(m)>\lim _{m \rightarrow \infty} B(m) 2 \lambda-5$. For $\lambda \in$ $(2.61,3], B(m)>0$ and $\frac{\partial A(1, m)}{\partial m}<0$, then $A(1, m)$ decreases as $m$ increases, $A(\mu, m)>$ $\lim _{m \rightarrow \infty} A(1, m)=0$ and (4.41) holds.

Therefore, (4.34) holds. By (4.25), $f_{\lambda} \underbrace{f_{\mu} \cdots f_{\mu}}_{k}(x)-x$ gets the minimum value at $x=1-\frac{1}{\mu}$ and $x=y *$, then because of (4.34) and $f_{\lambda} \underbrace{f_{\mu} \cdots f_{\mu}}_{k}\left(1-\frac{1}{\mu}\right)>1-\frac{1}{\mu},(2.32)$ holds.

Case (4). $k=5 m+3$ for $m \geqslant 5$. See Figure 4.18 , we will prove the following inequality:

$$
f_{\lambda}\left(f_{\mu}\left(\frac{1}{2}-\frac{1}{2 \sqrt[5 m+3]{\mu^{5 m+2} \lambda}}\right)\right)>\frac{\mu-\sqrt{\mu^{2}-2 \mu+2 \sqrt{\mu^{2}-2 \mu+2\left(\frac{\mu}{\lambda}\right)^{\frac{1}{2 m+3}}}}}{2 \mu} .
$$




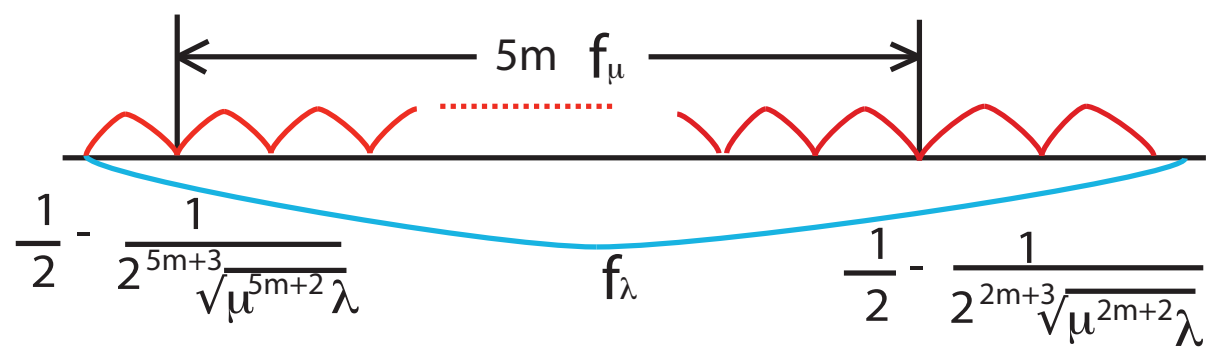

Figure 4.18: $f_{\lambda}\left(f_{\mu}\left(\frac{1}{2}-\frac{1}{2^{5 m+3} \sqrt{\mu^{5 m+2} \lambda}}\right)\right)>\frac{\mu-\sqrt{\mu^{2}-2 \mu+2 \sqrt{\mu^{2}-2 \mu+2\left(\frac{\mu}{\lambda}\right)^{\frac{1}{2 m+3}}}}}{2 \mu}$

Let

$A(\mu, m)=\left(\mu^{2}-\left(\frac{\mu}{\lambda}\right)^{\frac{2}{5 m+3}}\right)\left(4 \mu-\mu^{2}+\left(\frac{\mu}{\lambda}\right)^{\frac{2}{5 m+3}}\right)-\frac{8 \mu^{2}}{\lambda}+\frac{8 \mu}{\lambda} \sqrt{\mu^{2}-2 \mu+2 \sqrt{\mu^{2}-2 \mu+2\left(\frac{\mu}{\lambda}\right)^{\frac{1}{2 m+3}}}}$

and differentiate $A(\mu, m)$ as to $\mu$ to get

$$
\begin{aligned}
\frac{\partial A(\mu, m)}{2 \partial \mu}= & 6 \mu^{2}-2 \mu^{3}-\frac{8 \mu}{\lambda}+(2 \mu-2)\left(\frac{\mu}{\lambda}\right)^{\frac{2}{5 m+3}}+\frac{2 \mu}{5 m+3}\left(\frac{\mu}{\lambda}\right)^{\frac{2}{5 m+3}}-\frac{2}{(5 m+3) \mu}\left(\frac{\mu}{\lambda}\right)^{\frac{4}{5 m+3}} \\
& -\frac{4}{5 m+3}\left(\frac{\mu}{\lambda}\right)^{\frac{2}{5 m+3}}+\frac{4}{\lambda} \sqrt{\mu^{2}-2 \mu+2 \sqrt{\mu^{2}-2 \mu+2\left(\frac{\mu}{\lambda}\right)^{\frac{1}{2 m+3}}}} \\
& +\frac{4 \mu}{\lambda} \frac{\mu-1+\frac{\mu-1+\frac{1}{(2 m+3) \mu}\left(\frac{\mu}{\lambda}\right)^{\frac{1}{2 m+3}}}{\sqrt{\mu^{2}-2 \mu+2\left(\frac{\mu}{\lambda}\right)^{\frac{1}{2 m+3}}}}}{\sqrt{\mu^{2}-2 \mu+2 \sqrt{\mu^{2}-2 \mu+2\left(\frac{\mu}{\lambda}\right)^{\frac{1}{2 m+3}}}}}
\end{aligned}
$$

For $\mu \geqslant 5,\left(\frac{\mu}{\lambda}\right)^{\frac{1}{2 m+3}}>0.9189, \sqrt{\mu^{2}-2 \mu+2 \sqrt{\mu^{2}-2 \mu+2\left(\frac{\mu}{\lambda}\right)^{\frac{1}{2 m+3}}}}>0.911$ and then we can easily get $\frac{\sqrt{\mu^{2}-2 \mu+2 \sqrt{\mu^{2}-2 \mu+2\left(\frac{\mu}{\lambda}\right)^{\frac{1}{2 m+3}}}}}{\lambda}>\frac{0.91}{3}>\frac{1}{5 m+3}$. So $\frac{\partial A(\mu, m)}{\partial \mu}>0, A(\mu, m)>$ $A(1, m)$ and

$$
\begin{aligned}
A(1, m)= & 3-2 \lambda^{-\frac{2}{5 m+3}}-\lambda^{-\frac{4}{5 m+3}}-\frac{8}{\lambda}+\frac{8 \sqrt{-1+2 \sqrt{-1+2 \lambda^{-\frac{1}{2 m+2}}}}}{\lambda} \\
\frac{\partial A(1, m)}{\partial m}= & \frac{-4 \lambda^{-1-\frac{1}{2 m+3}} \ln \lambda}{(5 m+3)^{2} \sqrt{-1+2 \lambda^{-\frac{1}{2 m+3}}} \sqrt{-1+2 \sqrt{-1+2 \lambda^{-\frac{1}{2 m+3}}}}} \\
& \cdot\left[\lambda^{\frac{10 m^{2}+22 m+6}{(5 m+3)(2 m+3)}}\left(1+\lambda^{-\frac{2}{5 m+3}}\right) \sqrt{-1+2 \lambda^{-\frac{1}{2 m+3}}} \sqrt{-1+2 \sqrt{-1+2 \lambda^{-\frac{1}{2 m+3}}}}-\frac{4(5 m+3)^{2}}{5(2 m+3)^{2}}\right]
\end{aligned}
$$




$$
\begin{aligned}
&> \frac{-4 \lambda^{-1-\frac{1}{2 m+3}}(\ln \lambda) \cdot\left[\lambda^{\frac{10 m^{2}+22 m+6}{(5 m+3)(2 m+3)}}\left(1+\lambda^{-\frac{2}{5 m+3}}\right)\left(-1+2 \sqrt{-1+2 \lambda^{-\frac{1}{2 m+3}}}\right)-\frac{4(5 m+3)^{2}}{5(2 m+3)^{2}}\right.}{(5 m+3)^{2} \sqrt{-1+2 \lambda^{-\frac{1}{2 m+3}}} \sqrt{-1+2 \sqrt{-1+2 \lambda^{-\frac{1}{2 m+3}}}}} \\
&:=\frac{-4 \lambda^{-1-\frac{1}{2 m+3}} \cdot(\ln \lambda)}{(5 m+3)^{2} \sqrt{-1+2 \lambda^{-\frac{1}{2 m+3}}} \sqrt{-1+2 \sqrt{-1+2 \lambda^{-\frac{1}{2 m+3}}}}} B(m) .
\end{aligned}
$$

We differentiate $B(m)$ with respect to $m$ to get

$$
\begin{aligned}
B^{\prime}(m)= & \frac{1}{(5 m+3)^{2}}\left[\lambda ^ { \frac { 1 0 m ^ { 2 } + 2 2 m + 6 } { ( 5 m + 3 ) ( 2 m + 3 ) } } \operatorname { l n } \lambda \left(\frac{-10 m^{2}+60 m+72}{(2 m+3)^{2}}\left(1+\lambda^{-\frac{2}{5 m+3}}\right)\left(-1+2 \sqrt{-1+2 \lambda^{-\frac{1}{2 m+3}}}\right)\right.\right. \\
& +10 \lambda^{\frac{-2}{5 m+3}}\left(-1+2 \sqrt{-1+2 \lambda^{-\frac{1}{2 m+3}}}\right) \\
& \left.\left.+\frac{4\left(\lambda^{-\frac{1}{2 m+3}}+\lambda^{-\frac{9 m+9}{(5 m+3)(2 m+3)}}\right)(5 m+3)^{2}}{(2 m+3)^{2} \sqrt{-1+2 \lambda^{-\frac{1}{2 m+3}}}}\right)-\frac{72(5 m+3)^{3}}{5(2 m+3)^{3}}\right] .
\end{aligned}
$$

Since $\frac{-10 m^{2}+60 m+72}{(2 m+3)^{2}}<0.722, \lambda^{\frac{10 m^{2}+2 m+6}{(5 m+3)(2 m+3)}} \ln \lambda<3.32, \sqrt{-1+2 \lambda^{-\frac{1}{2 m+3}}}>0.915$ and $\left(3.32 \frac{8(5 m+3)^{2}}{0.915(2 m+3)^{2}}-\frac{72(5 m+3)^{3}}{5(2 m+3)^{3}}\right)_{m}^{\prime}=\frac{72(5 m+3)}{(2 m+3)^{3}}\left(\frac{6.64}{0.915}-\frac{27(5 m+3)}{5(2 m+3)}\right)<0$, we can get

$$
\begin{aligned}
B^{\prime}(m) & <\frac{1}{(5 m+3)^{2}}\left[3.32 \cdot\left(0.722 \cdot 2+10+\frac{8(5 m+3)^{2}}{0.915(2 m+3)^{2}}\right)-\frac{72(5 m+3)^{3}}{5(2 m+3)^{3}}\right], \\
& <\frac{1}{(5 m+3)^{2}}\left[3.32 \times 10.444+3.32 \frac{50}{0.914}-225\right]<0 .
\end{aligned}
$$

So $B(m)$ decreases as $m$ increases and $B(m)>\lim _{m \rightarrow \infty} B(m)=2 \lambda-5$. When $\lambda \in[2.61,3]$, $B(m)>0, \frac{\partial A(1, m)}{\partial m}<0$ and $A(1, m)$ is a decreasing function as to $m$, so we can get $A(\mu, m)>\lim _{m \rightarrow \infty} A(1, m)=0$ and (4.42) holds.

So (4.34) holds. By (4.25), $f_{\lambda} \underbrace{f_{\mu} \cdots f_{\mu}}_{k}(x)-x$ reaches the minimum value at $x=1-\frac{1}{\mu}$ and $x=y *$, then because of $(4.34)$ and $f_{\lambda} \underbrace{f_{\mu} \cdots f_{\mu}}_{k}\left(1-\frac{1}{\mu}\right)>1-\frac{1}{\mu},(2.32)$ holds.

Case (5), $k=5 m+4$ for $m \geqslant 4$. See Figure 4.19, we need to prove the following inequality

$$
f_{\lambda}\left(f_{\mu}\left(\frac{1}{2}-\frac{1}{2 \sqrt[5 m+4]{\mu^{5 m+3} \lambda}}\right)\right)>\frac{1}{2}-\frac{1}{2 \sqrt[2 m+2]{\mu^{2 m+1} \lambda}} .
$$

Let $A(\mu, m)=\left(\mu^{2}-\left(\frac{\mu}{\lambda}\right)^{\frac{2}{5 m+4}}\right)\left(4 \mu-\mu^{2}+\left(\frac{\mu}{\lambda}\right)^{\frac{2}{5 m+4}}\right)-\frac{8 \mu^{2}}{\lambda}+8\left(\frac{\mu}{\lambda}\right)^{\frac{2 m+3}{2 m+2}}$ and differentiate $A(\mu, m)$ as to $\mu$ to get

$$
\begin{aligned}
\frac{\partial A(\mu, m)}{2 \partial \mu}= & 6 \mu^{2}-2 \mu^{3}-\frac{8 \mu}{\lambda}+(2 \mu-2)\left(\frac{\mu}{\lambda}\right)^{\frac{2}{5 m+4}}+\frac{2 \mu}{5 m+4}\left(\frac{\mu}{\lambda}\right)^{\frac{2}{5 m+4}}-\frac{2}{(5 m+4) \mu}\left(\frac{\mu}{\lambda}\right)^{\frac{4}{5 m+4}} \\
& -\frac{4}{5 m+4}\left(\frac{\mu}{\lambda}\right)^{\frac{2}{5 m+4}}+4 \frac{2 m+3}{(2 m+2) \lambda}\left(\frac{\mu}{\lambda}\right)^{\frac{1}{2 m+2}} .
\end{aligned}
$$




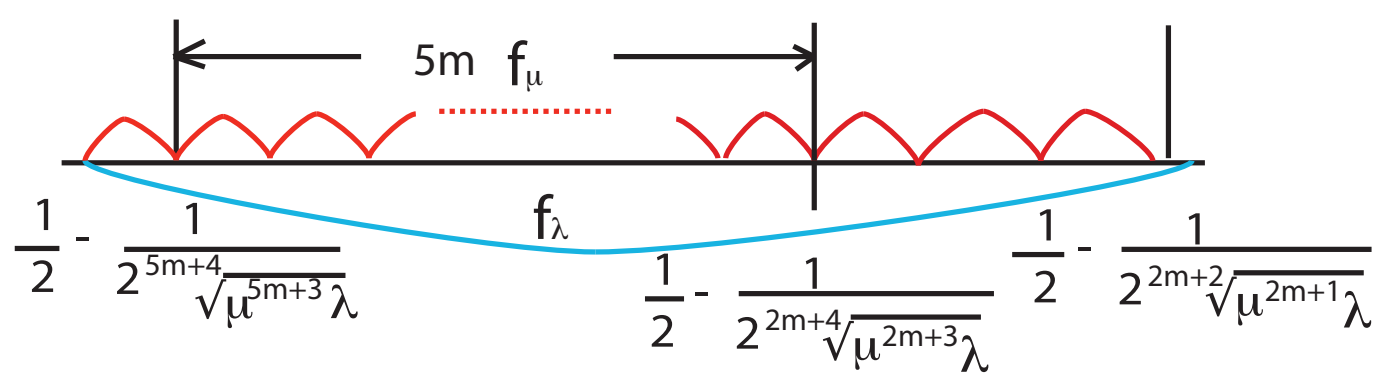

Figure 4.19: $f_{\lambda}\left(f_{\mu}\left(\frac{1}{2}-\frac{1}{2 \sqrt[5 m+4]{\mu^{5 m+3} \lambda}}\right)\right)>\frac{1}{2}-\frac{1}{2 \sqrt[2 m+2]{\mu^{2 m+1} \lambda}}$

Since $6 \mu^{2}-2 \mu^{3}-\frac{8 \mu}{\lambda}>2 \mu\left(3 \mu-\mu^{2}-1\right)>0,\left(\frac{\lambda}{\mu}\right)^{\frac{m}{(2 m+2)(5 m+4)}}<1.02$ and $\frac{\left(\frac{\mu}{\lambda}\right)^{\frac{1}{2 m+2}}}{\lambda}-\frac{\left(\frac{\mu}{\lambda}\right)^{\frac{2}{5 m+4}}}{5 m+4}=$ $\frac{\left(\frac{\mu}{\lambda}\right)^{\frac{1}{2 m+2}}}{\lambda}\left(1-\frac{\lambda\left(\frac{\lambda}{\mu}\right)^{\frac{m}{(2 m+2)(5 m+4)}}}{5 m+4}\right)>0$, we can easily get $\frac{\partial A(\mu, m)}{\partial \mu}>0$ and $A(\mu, m)$ increases as $\mu$ increases,

$$
A(\mu, m)>A(1, m)=3-2 \lambda^{-\frac{2}{5 m+4}}-\lambda^{-\frac{4}{5 m+4}}-\frac{8}{\lambda}+8 \lambda^{-\frac{2 m+3}{2 m+2}} .
$$

Since $\frac{16(5 m+4)^{3}}{5(2 m+2)^{3}}>44.2$ for $m \geqslant 5$, we have

$\frac{\partial A(1, m)}{\partial m}=\frac{-5 \lambda^{-\frac{2 m+3}{2 m+2}} \ln \lambda}{(5 m+4)^{2}}\left[\lambda^{\frac{10 m^{2}+19 m+8}{(5 m+4)(2 m+2)}}\left(1+\lambda^{-\frac{2}{5 m+4}}\right)-\frac{4(5 m+4)^{2}}{5(2 m+2)^{2}}\right]=\frac{-5 \lambda^{-\frac{2 m+3}{2 m+2}} \ln \lambda}{(5 m+4)^{2}} B(m)$.

We differentiate $B(m)$ with respect to $m$ to get

$$
\begin{aligned}
B^{\prime}(m)= & \lambda^{\frac{10 m^{2}+19 m+8}{(5 m+4)(2 m+2)}} \frac{-10 m^{2}+8}{(2 m+2)^{2}(5 m+4)^{2}} \ln \lambda\left(1+\lambda^{-\frac{2}{5 m+4}}\right) \\
& +\lambda^{\frac{10 m^{2}+15 m+4}{(5 m+4)(2 m+2)}} \frac{10}{(5 m+4)^{2}} \ln \lambda-\frac{16(5 m+4)}{5(2 m+2)^{3}} \\
< & \frac{1}{(5 m+4)^{2}}\left[10 \lambda \ln \lambda-\frac{16(5 m+4)^{3}}{5(2 m+2)^{3}}\right]<\frac{1}{(5 m+4)^{2}}(30 \ln 3-44.2)<0 .
\end{aligned}
$$

So $B(m)$ decreases as $m$ increases and $B(m)>\lim _{m \rightarrow \infty}=2 \lambda-5>0$ for $\lambda \in(2.61,3]$. Therefore, $\frac{\partial A(1, m)}{\partial m}<0$ and we can get

$$
A(\mu, m)>\lim _{m \rightarrow \infty} A(1, m)=0 .
$$

So (4.22) holds. By (4.25), $f_{\lambda} \underbrace{f_{\mu} \cdots f_{\mu}}_{k}(x)-x$ attains the minimum value at $x=1-\frac{1}{\mu}$ and $x=b_{k}$, then because of $(4.34)$ and $f_{\lambda} \underbrace{f_{\mu} \cdots f_{\mu}}_{k}\left(1-\frac{1}{\mu}\right)>1-\frac{1}{\mu},(2.32)$ holds.

Concluding Case (I) and Case (II), Lemma 2.1.13 holds for all $\mu \in\left(1, \frac{4}{\lambda}\right], \lambda \in(2,3]$ and $k \geqslant 2$. 


\section{$4.53<\mu<\lambda \leqslant 1+\sqrt{5}$}

Proof of Lemma 2.2.1. Since $\mu \geqslant 3.00547$, so $\beta_{\mu} \geqslant 0.690988$. Under this condition, $\beta_{\mu} \geqslant 1-\frac{1}{1+\sqrt{5}} \geqslant 1-\frac{1}{\lambda}$ holds for any $\lambda \leqslant 1+\sqrt{5}$. Because $F_{\mu}\left(x_{1}\right)>x_{2}$ is obviously true when $\beta_{\mu} \geqslant x_{2}$, i.e. $\mu \geqslant 3.05018$. So we just need to discuss the case when $\mu \in$ $[3.00547,3.05018]$. In this case, $F_{\lambda}^{\prime}\left(\beta_{\mu}\right)\left(F_{\mu}^{\prime}\left(\beta_{\mu}\right)\right)^{20}>1+\sqrt{5}$ when $\mu=3.00547$, hence $k-1 \leqslant 20$, so $k \leqslant 21$.

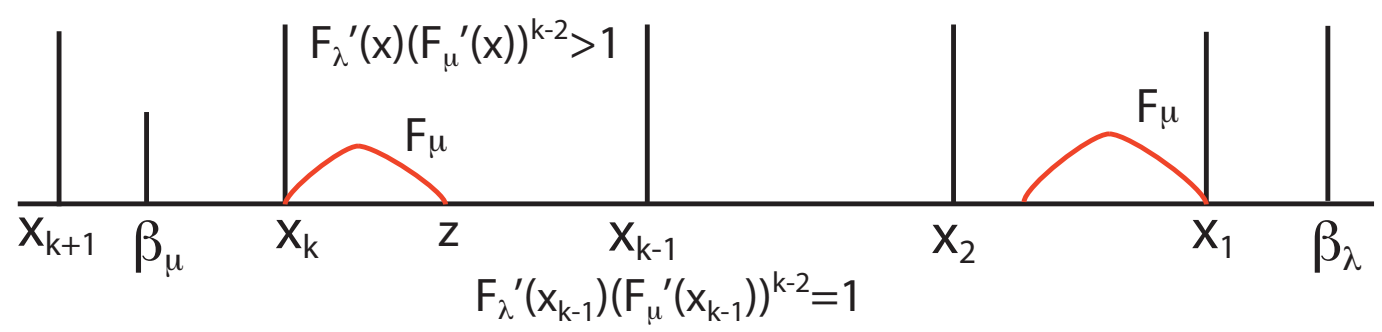

Figure 4.20: $F_{\mu}\left(x_{k-1}\right)>x_{k}$

If $F_{\mu}(z)=x_{k}$, then $z=\frac{\mu+\sqrt{\mu^{2}-2 \mu-2 \sqrt{\mu^{2}-4 \mu x_{k}}}}{2 \mu}$. In order to prove (2.46), we just need to prove the following inequality as Figure 4.20 shows,

$$
F_{\lambda}^{\prime}(z)\left(F_{\mu}^{\prime}(z)\right)^{k-2}>1 .
$$

This means that we will prove the following inequality

$$
\left(\sqrt{\mu^{2}-2 \mu-2 \sqrt{\mu^{2}-4 \mu x_{k}}} \sqrt{\mu^{2}-4 \mu x_{k}}\right)^{k-1}>\frac{\mu \sqrt{\mu^{2}-4 \mu x_{k}}}{\lambda^{3}\left(\mu+\sqrt{\mu^{2}-4 \mu x_{k}}\right)-\lambda^{2} \mu^{2}} .
$$

Because $\frac{\mu \sqrt{\mu^{2}-4 \mu x_{k}}}{\lambda^{3}\left(\mu+\sqrt{\mu^{2}-4 \mu x_{k}}\right)-\lambda^{2} \mu^{2}}<\frac{\mu}{\lambda^{3}}<\frac{1}{\mu^{2}}$, we just need to prove the following inequality

$$
A\left(\mu, x_{k}, k\right)=\left(\sqrt{\mu^{2}-2 \mu-2 \sqrt{\mu^{2}-4 \mu x_{k}}} \sqrt{\mu^{2}-4 \mu x_{k}}\right)^{k-1}-\frac{1}{\mu^{2}}>0 .
$$

Note

$$
\begin{aligned}
\frac{\partial A\left(\mu, x_{k}, k\right)}{\partial x_{k}}= & -(k-1)\left(\sqrt{\mu^{2}-2 \mu-2 \sqrt{\mu^{2}-4 \mu x_{k}}} \sqrt{\mu^{2}-4 \mu x_{k}}\right)^{k-2} \\
& \frac{2 \mu}{\sqrt{\mu^{2}-2 \mu-2 \sqrt{\mu^{2}-4 \mu x_{k}}} \sqrt{\mu^{2}-4 \mu x_{k}}}\left(\mu^{2}-2 \mu-3 \sqrt{\mu^{2}-4 \mu x_{k}}\right), \\
\frac{\partial A\left(\mu, x_{k}, k\right)}{\partial \mu}= & \frac{(k-1)\left(\sqrt{\mu^{2}-2 \mu-2 \sqrt{\mu^{2}-4 \mu x_{k}}} \sqrt{\mu^{2}-4 \mu x_{k}}\right)^{k-2}}{\sqrt{\mu^{2}-2 \mu-2 \sqrt{\mu^{2}-4 \mu x_{k}}} \sqrt{\mu^{2}-4 \mu x_{k}}} \\
& {\left[(\mu-1)\left(\mu^{2}-4 \mu x_{k}\right)+\left(\mu-2 x_{k}\right)\left(\mu^{2}-2 \mu-3 \sqrt{\mu^{2}-4 \mu x_{k}}\right)\right]+\frac{2}{\mu^{3}} . }
\end{aligned}
$$


If $\sqrt{\mu^{2}-2 \mu-2 \sqrt{\mu^{2}-4 \mu x_{k}}} \sqrt{\mu^{2}-4 \mu x_{k}}>1$, then (4.45) is evident. Otherwise, $A\left(\mu, x_{k}, k\right)$ is decreasing with respect to $k$. Meanwhile, since $\sqrt{\mu^{2}-4 \mu x_{k}}<\sqrt{3.05018^{2}-4 \cdot 3.05 \cdot 0.690988}<$ 1 and $\mu^{2}-2 \mu-3 \sqrt{\mu^{2}-4 \mu x_{k}}=(\mu-1)^{2}-1-3 \sqrt{\mu^{2}-4 \mu x_{k}}>0$ for $\mu \in[3.00547,3.05018]$ and $x_{k} \in[0.690988,0.7378252]$, we can get that $\frac{\partial A\left(\mu, x_{k}\right)}{\partial x_{k}}<0$ and $\frac{\partial A\left(\mu, x_{k}\right)}{\partial \mu}>0$. Therefore, $A\left(\mu, x_{k}\right)$ increases as $\mu$ increases and $x_{k}$ decreases. So we have the following conclusions:

(i) When $k \leqslant 5, x_{k} \leqslant 0.7378252, A\left(\mu, x_{k}, k\right)>A(3.00547,0.7378252,5)>0$;

(ii) When $k \in[6,15], \mu \leqslant 3.02166, x_{k} \leqslant 0.71431$,

$$
A\left(\mu, x_{k}, k\right)>A(3.00547,0.71431,15)>0
$$

(iii) When $k \in[16,21], \mu \leqslant 3.00603$ and $x_{k} \leqslant 0.6922$,

$$
A\left(\mu, x_{k}, k\right)>A(3.00547,0.6922,21)>0 .
$$

As a result, (2.46) holds and Lemma 2.2.1 is proved.

Proof of Lemma 2.2.2. First, solving $F_{\lambda}^{\prime}\left(x_{1}\right)=1$, we can get

$$
x_{1}(\lambda)=\frac{1}{2}+\sqrt{\frac{\lambda-2}{3 \lambda}} \cos \left(\frac{1}{3} \arccos \frac{3}{\lambda(2-\lambda)} \sqrt{\frac{3}{\lambda(\lambda-2)}}\right) .
$$

Set $A(\lambda)=F_{\lambda}\left(F_{\mu}\left(x_{1}(\lambda)\right)\right)-x_{1}(\lambda)$. We only consider the case when $\mu=3$. If this is true for $A(\lambda)>0$, then $A(\lambda)>0$ for all $\mu \geqslant 3.00547$.

$$
\begin{aligned}
A(\lambda)= & 9 x_{1}(\lambda)\left[\lambda^{2}\left(1-x_{1}(\lambda)\right)\left(1-3 x_{1}(\lambda)+3 x_{1}(\lambda)^{2}\right)\left(1-9 x_{1}(\lambda)\left(1-x_{1}(\lambda)\right)\left(1-3 x_{1}(\lambda)+3 x_{1}^{2}(\lambda)\right)\right)\right. \\
& \left(1-9 \lambda x_{1}(\lambda)\left(1-x_{1}(\lambda)\right)\left(1-3 x_{1}(\lambda)+3 x_{1}^{2}(\lambda)\right)\right. \\
& \left.\left.+81 \lambda x_{1}^{2}(\lambda)\left(1-x_{1}(\lambda)\right)^{2}\left(1-3 x_{1}(\lambda)+3 x_{1}^{2}(\lambda)\right)^{2}\right)-\frac{1}{9}\right] .
\end{aligned}
$$

We use maple to differentiate $A(\lambda)$ with respect to $\lambda$ and observe $A^{\prime}(\lambda)>0$. We don't include the computation as it is quite lengthy. So we have

$$
A(\lambda)>A(3)=0 .
$$

So (2.47) holds for $x=x_{1}$. Note when for $x \in\left[\beta_{\mu}, x_{1}\right], F_{\lambda}^{\prime}(x) F_{\mu}^{\prime}(x)<1$. Let $B(x)=$ $F_{\lambda}\left(F_{\mu}(x)\right)-x$. Then

$$
\begin{aligned}
B_{x}^{\prime} & =F_{\lambda}^{\prime}\left(F_{\mu}(x)\right) F_{\mu}^{\prime}(x)-1 \quad\left(F_{\mu}(x)<x\right) \\
& <F_{\lambda}^{\prime}\left(F_{\mu}(x)\right) F_{\mu}^{\prime}\left(F_{\mu}(x)\right)-1 \quad\left(F_{\mu}(x) \geqslant \beta_{\mu}>x_{2}\right) \\
& <0 .
\end{aligned}
$$

So $B(x)$ is a decreasing function as to $\mu$ and $B(x)>B\left(x_{1}\right)>0$. Therefore, Lemma 2.2.2 holds. 


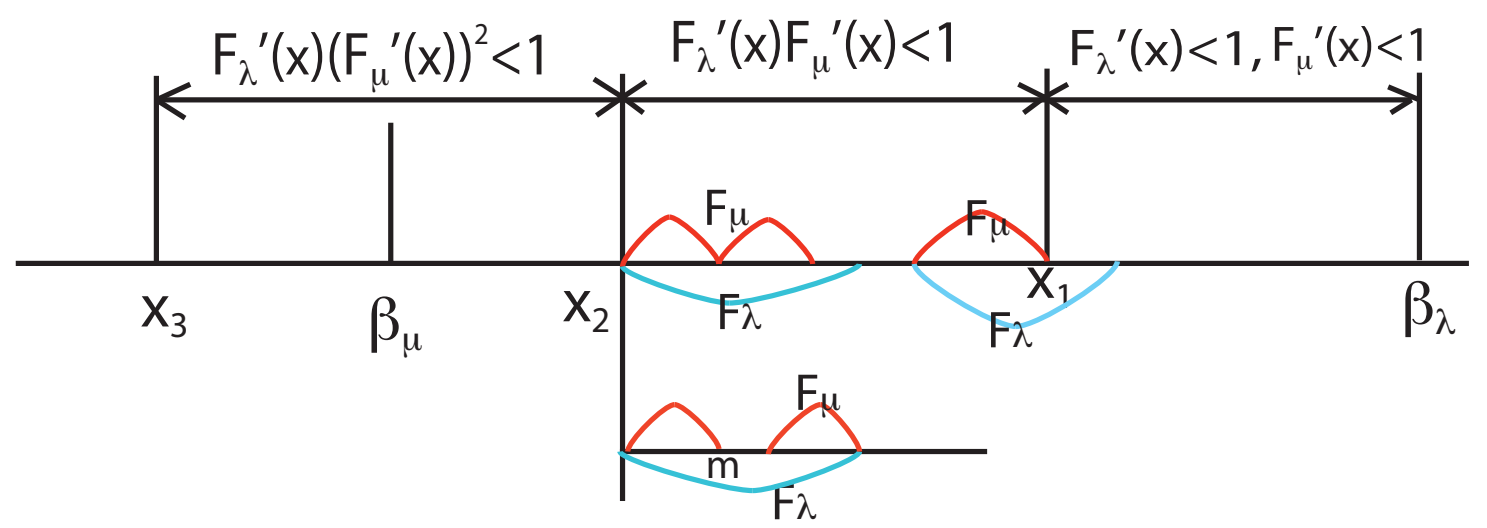

Figure 4.21: $\beta_{\mu} \in\left(x_{3}, x_{2}\right]$

Proof of Lemma 2.2.3. First, we will prove (2.48) holds for $x=y_{2}$. For this, we first need to prove $F_{\mu}\left(F_{\lambda}\left(x_{2}\right)\right)-m>0$, where $F_{\mu}(m)=x_{2}$. Note $m=\frac{\mu+\sqrt{\mu^{2}-2 \mu-2 \sqrt{\mu^{2}-4 \mu x_{2}}}}{2 \mu}$ as Figure 4.21 shows. Let $A\left(\mu, \lambda, x_{2}\right)=F_{\mu}\left(F_{\lambda}\left(x_{2}\right)\right)-m$. Then we get

$$
\begin{aligned}
A\left(\mu, \lambda, x_{2}\right)= & \mu^{2} \lambda^{2} x_{2}\left(1-x_{2}\right)\left(1-\lambda x_{2}+\lambda x_{2}^{2}\right)\left(1-\lambda^{2} x_{2}\left(1-x_{2}\right)\left(1-\lambda x_{2}+\lambda x_{2}^{2}\right)\right) \\
& \left(1-\mu \lambda^{2} x_{2}\left(1-x_{2}\right)\left(1-\lambda x_{2}+\lambda x_{2}^{2}\right)+\mu\left(\lambda^{2} x_{2}\left(1-x_{2}\right)\left(1-\lambda x_{2}+\lambda x_{2}^{2}\right)\right)^{2}\right) \\
& -\frac{\mu+\sqrt{\mu^{2}-2 \mu-2 \sqrt{\mu^{2}-4 \mu x_{2}}}}{2 \mu} .
\end{aligned}
$$

It is easy to see that

$$
\begin{aligned}
& \frac{\partial A\left(\mu, \lambda, x_{2}\right)}{\partial \lambda}=\frac{\partial F_{\mu}\left(F_{\lambda}\left(x_{2}\right)\right)}{\partial\left(F_{\lambda}\left(x_{2}\right)\right)} \frac{\partial F_{\lambda}\left(x_{2}\right)}{\partial \lambda} \\
& \frac{\partial^{2} A\left(\mu, \lambda, x_{2}\right)}{\partial \lambda^{2}}=\frac{\partial^{2} F_{\mu}\left(F_{\lambda}\left(x_{2}\right)\right)}{\partial\left(F_{\lambda}\left(x_{2}\right)\right)^{2}}\left(\frac{\partial F_{\lambda}\left(x_{2}\right)}{\partial \lambda}\right)^{2}+\frac{\partial F_{\mu}\left(F_{\lambda}\left(x_{2}\right)\right)}{\partial\left(F_{\lambda}\left(x_{2}\right)\right)} \frac{\partial^{2} F_{\lambda}\left(x_{2}\right)}{\partial \lambda^{2}} .
\end{aligned}
$$

Note $\frac{\partial F(\lambda, x)}{\partial \lambda}=\lambda x(1-x)\left(2-3 \lambda x+3 \lambda x^{2}\right)$ and $\frac{\partial^{2} F_{\lambda}\left(x_{2}\right)}{\partial \lambda^{2}}=2 x(1-x)\left(1-3 \lambda x+3 \lambda x^{2}\right)$. Moreover, $\frac{\partial F(\mu, x)}{\partial x}=\mu^{2}(1-2 x)\left(1-2 \mu x+2 \mu x^{2}\right)$ and $\frac{\partial^{2} F(\mu, x)}{\partial x^{2}}=-2 \mu^{2}\left(6 \mu x^{2}-6 \mu x+\mu+1\right)$. it is easy to see $1-3 \lambda x+3 \lambda x^{2}<0$ for $\lambda \in(3,1+\sqrt{5}],(1-2 x)\left(1-2 \mu x+2 \mu x^{2}\right)>0$ and $6 \mu x^{2}-6 \mu x+\mu+1>0$ when $\mu<1+\sqrt{5}$ and $x_{2}>0.690988$. Therefore, $\frac{\partial^{2} A\left(\mu, \lambda, x_{2}\right)}{\partial \lambda^{2}}<0$ and $A\left(\mu, \lambda, x_{2}\right)$ reaches the minimum point when $\lambda$ is at the start point or end point. Moreover,

$$
\begin{aligned}
\frac{\partial A\left(\mu, \lambda, x_{2}\right)}{\partial \mu}= & \lambda^{2} x_{2}\left(1-x_{2}\right)\left(1-\lambda x_{2}+\lambda x_{2}^{2}\right)\left(1-\lambda^{2} x_{2}\left(1-x_{2}\right)\left(1-\lambda x_{2}+\lambda x_{2}^{2}\right)\right) \\
& \mu\left(2-3 \mu \lambda^{2} x_{2}\left(1-x_{2}\right)\left(1-\lambda x_{2}+\lambda x_{2}^{2}\right)+3 \mu\left(\lambda^{2} x_{2}\left(1-x_{2}\right)\left(1-\lambda x_{2}+\lambda x_{2}^{2}\right)^{2}\right)\right. \\
& -\frac{\mu\left(1-\frac{\mu-2 x_{2}}{\sqrt{\mu^{2}-4 \mu x_{2}}}\right)+2 \sqrt{\mu^{2}-4 \mu x_{2}}}{2 \mu^{2} \sqrt{\mu^{2}-2 \mu-2 \sqrt{\mu^{2}-4 \mu x_{2}}}} .
\end{aligned}
$$


Since $\mu \in[3.00547,3.05018]$, we have $x_{2}>\beta_{\mu}>0.690988$, and $\mu \sqrt{\mu^{2}-4 \mu x_{2}}-\mu\left(\mu-2 x_{2}\right)+$ $2\left(\mu^{2}-4 \mu x_{2}\right)=\mu \sqrt{\mu^{2}-4 \mu x_{2}}+\mu^{2}-6 \mu x_{2}<\mu\left(\mu-6 \cdot 0.690988+\sqrt{\mu^{2}-4 \mu \cdot 0.690988}\right)<0$. So it is evident that $\frac{\partial A\left(\mu, \lambda, x_{2}\right)}{\partial \mu}>0$ and $A\left(\mu, \lambda, x_{2}\right)$ increases as $\mu$ increases. Moreover,

$$
\begin{aligned}
\frac{\partial A\left(\mu, \lambda, x_{2}\right)}{\partial x_{2}}= & F_{\lambda}\left(x_{2}\right)^{\prime} F_{\mu}^{\prime}\left(F_{\lambda}\left(x_{2}\right)\right)-\frac{\partial m}{\partial x_{2}} \\
= & \mu^{2} \lambda^{2}\left(1-2 x_{2}\right)\left(1-2 \lambda x_{2}+2 \lambda x_{2}^{2}\right)\left(1-2 F_{\lambda}\left(x_{2}\right)\right)\left(1-2 \mu F_{\lambda}\left(x_{2}\right)+2 \mu\left(F_{\lambda}\left(x_{2}\right)\right)^{2}\right) \\
& -\frac{1}{\sqrt{\left(\mu^{2}-2 \mu-2 \sqrt{\mu^{2}-4 \mu x_{2}}\right)\left(\mu^{2}-4 \mu x_{2}\right)}}, \\
\frac{\partial^{2} A\left(\mu, \lambda, x_{2}\right)}{\partial x_{2}{ }^{2}}= & -2 \mu^{2} \lambda^{2}\left(1+\lambda+6 \lambda x_{2}^{2}-6 \lambda x_{2}\right)\left(1-2 F_{\lambda}\left(x_{2}\right)\right)\left(1-2 \mu F_{\lambda}\left(x_{2}\right)+2 \mu\left(F_{\lambda}\left(x_{2}\right)\right)^{2}\right) \\
& -2 \mu^{2} \lambda^{2}\left(1-2 x_{2}\right)\left(1-2 \lambda x_{2}+2 \lambda x_{2}^{2}\right)\left(1+\mu+6 \mu\left(F_{\lambda}\left(x_{2}\right)\right)^{2}-6 \mu F_{\lambda}\left(x_{2}\right)\right) F_{\lambda}^{\prime}\left(x_{2}\right) \\
& +\frac{1}{2}\left[\left(\mu^{2}-2 \mu-2 \sqrt{\mu^{2}-4 \mu x_{2}}\right)\left(\mu^{2}-4 \mu x_{2}\right)\right]^{-\frac{3}{2}}(-4 \mu)\left(\mu^{2}-2 \mu-3 \sqrt{\mu^{2}-4 \mu x_{2}}\right) .
\end{aligned}
$$

Since $F_{\lambda}^{\prime}\left(x_{2}\right)>0, x_{2}>0.690988$, so

$$
\begin{aligned}
6 \lambda x^{2}-6 \lambda x+\lambda+1 & =6 \lambda\left(x-\frac{1}{2}\right)^{2}-\frac{\lambda}{2}+1>1-0.2603 \lambda>0 \\
\left(\mu^{2}-2 \mu\right)^{2}-\left(3 \sqrt{\mu^{2}-4 \mu x_{1}}\right)^{2} & =\mu\left[\mu(\mu-2)^{2}-9 \mu+36 x_{2}\right] \\
& \geqslant \mu\left[\mu^{3}-4 \mu^{2}-5 \mu+24.87\right]>0 .
\end{aligned}
$$

Thus $\frac{\partial^{2} A\left(\mu, \lambda, x_{2}\right)}{\partial x_{2}{ }^{2}}<0$ and it follows that $A\left(\mu, \lambda, x_{2}\right)$ reaches the minimum point at the start point or the end point of $x_{2}$ for some given $\mu$ and $\lambda$.

Now, let $D(\mu, \lambda, k)=F_{\lambda}^{\prime}\left(\beta_{\mu}\right)\left(F_{\mu}^{\prime}\left(\beta_{\mu}\right)\right)^{k}=\frac{2 \lambda}{\mu^{3}}(1+\sqrt{(\mu+1)(\mu-3)})[\lambda(\mu+1-\sqrt{(\mu+1)(\mu-3)})-$ $\left.\mu^{2}\right]\left(-\mu^{2}+2 \mu+4\right)^{k}$. Then

$$
\begin{aligned}
\frac{\partial D(\mu, \lambda, k)}{\partial \lambda}= & \frac{2 \lambda}{\mu^{3}}(1+\sqrt{(\mu+1)(\mu-3)})\left[\lambda(\mu+1-\sqrt{(\mu+1)(\mu-3)})-\mu^{2}\right]\left(-\mu^{2}+2 \mu+4\right)^{k} \\
& +\frac{\lambda^{2}}{\mu^{3}}(1+\sqrt{(\mu+1)(\mu-3)})[\mu+1-\sqrt{(\mu+1)(\mu-3)}]\left(-\mu^{2}+2 \mu+4\right)^{k} \\
> & 0, \\
\frac{\partial D(\mu, \lambda, k)}{\partial \mu}= & -3 \frac{\lambda^{2}}{\mu^{4}}(1+\sqrt{(\mu+1)(\mu-3)})\left[\lambda(\mu+1-\sqrt{(\mu+1)(\mu-3)})-\mu^{2}\right]\left(-\mu^{2}+2 \mu+4\right)^{k} \\
& +\frac{2 \lambda}{\mu^{3}}\left(-\mu^{2}+2 \mu+4\right)^{k}\left(\frac{\mu-1}{\sqrt{(\mu+1)(\mu-3)}}\left(\lambda(\mu+1-\sqrt{(\mu+1)(\mu-3)})-\mu^{2}\right)\right) \\
& \frac{2 \lambda}{\mu^{3}}(1+\sqrt{(\mu+1)(\mu-3)})\left(-\mu^{2}+2 \mu+4\right)^{k}\left(\lambda\left(1-\frac{\mu-1}{\sqrt{(\mu+1)(\mu-3)}}\right)-2 \mu\right) \\
& +\frac{4 k \lambda}{\mu^{3}}(1+\sqrt{(\mu+1)(\mu-3)})\left[\lambda(\mu+1-\sqrt{(\mu+1)(\mu-3)})-\mu^{2}\right] \\
& \left(-\mu^{2}+2 \mu+4\right)^{k-1}(1-\mu) \\
< & \frac{2 \lambda(\mu-1)\left(-\mu^{2}+2 \mu+4\right)^{k}}{\mu^{3} \sqrt{(\mu+1)(\mu-3)}}
\end{aligned}
$$




$$
\begin{aligned}
& \cdot\left[\lambda(\mu+1-\sqrt{(\mu+1)(\mu-3)})-\mu^{2}-\lambda(1+\sqrt{(\mu+1)(\mu-3)})\right] \\
& \frac{2 \lambda(\mu-1)\left(-\mu^{2}+2 \mu+4\right)^{k}}{\mu^{3} \sqrt{(\mu+1)(\mu-3)}}(-2 \lambda \sqrt{(\mu+1)(\mu-3)}+\mu(\lambda-\mu)) .
\end{aligned}
$$

Since it is easy to see that $-2 \lambda \sqrt{(\mu+1)(\mu-3)}+\mu(\lambda-\mu)$ is an increasing function with respect to $\lambda$ and a decreasing function with respect to $\mu$, then for $3.00547 \leqslant \mu<\lambda \leqslant$ $1+\sqrt{5}$, we have

$-2 \lambda \sqrt{(\mu+1)(\mu-3)}+\mu(\lambda-\mu)<-2 \lambda \sqrt{(3.00547+1)(3.00547-3)}+3.00547(1+\sqrt{5}-3.00547)<0$.

Consequently, $\frac{\partial D(\mu, \lambda, k)}{\partial \mu}<0$, so $D(\mu, \lambda, k)$ increases as $\lambda$ increases and decreases as $\mu$ increases. So if we can make sure $D(\mu, 1+\sqrt{5}, k)<1$, then $D(\mu, \lambda, k)<1$. When $3.05018 \leqslant \mu<\lambda \leqslant 1+\sqrt{5}, D(\mu, 1+\sqrt{5}, 1)<1$. Note this case was already considered before. As a result, for $k=2$, we just need to discuss the value of $A\left(x_{2}\right)$ when $\mu \in$ $[3.00547,3.05018)$ and $\lambda \in(\mu, 1+\sqrt{5}]$. We can get the following cases.

(i) $\mu \in[3.025,3.05018]$. It is easy to know if $\lambda \leqslant 3.16668, D(\mu, \lambda, 2)>1$. But $D(\mu, \lambda, 2)<1$, thus $\lambda \in[3.16668,1+\sqrt{5}]$ and then we can get $x_{2} \in[0.690988,0.7378252]$,

$$
\begin{aligned}
A\left(\mu, \lambda, x_{2}\right)> & A(3.025,3.16668,0.7378252)>0, \\
A\left(\mu, \lambda, x_{2}\right)> & A(3.025,3.16668,0.0 .690988)>0, \\
A\left(\mu, \lambda, x_{2}\right)> & A(3.025,1+\sqrt{5}, 0.378252)>0, \\
A\left(\mu, \lambda, x_{2}\right)> & A(3.025,1+\sqrt{5}, 0.690988)>0, \\
A\left(\mu, \lambda, x_{2}\right)> & \min \{A(3.025,3.16668,0.7378252), A(3.025,3.16668,0.690988) \\
& A(3.025,1+\sqrt{5}, 0.378252), A(3.025,1+\sqrt{5}, 0.690988)\}>0 .
\end{aligned}
$$

(ii) $\mu \in[3.015,3.025)$, we know if $\lambda \leqslant 3.0995, D(\mu, \lambda, 2)>1$. But in our case, $D(\mu, \lambda, 2)<1$, so we have $\lambda \in[3.0995,1+\sqrt{5}]$ and we can get $x_{2} \in[0.690988,0.734151]$.

$$
\begin{aligned}
& A\left(\mu, \lambda, x_{2}\right)>A(3.015,3.171,0.734151)>0, \text { for } \lambda \in(3.171,1+\sqrt{5}], \\
& A\left(\mu, \lambda, x_{2}\right)>A(3.015,3.0995,0.72604)>0, \text { for } \lambda \in[3.0995,3.171], \\
& A\left(\mu, \lambda, x_{2}\right)>A(3.015,3.0995,0.690988)>0, \\
& A\left(\mu, \lambda, x_{2}\right)>A(3.015,1+\sqrt{5}, 0.734151)>0, \\
& A\left(\mu, \lambda, x_{2}\right)>A(3.015,1+\sqrt{5}, 0.690988)>0, \\
& A\left(\mu, \lambda, x_{2}\right)>\min \{A(3.015,3.171,0.734151), A(3.015,3.0995,0.72604) \\
& A(3.015,3.0995,0.690988), A(3.015,1+\sqrt{5}, 0.734151), A(3.015,1+\sqrt{5}, 0.690988)\}>0 .
\end{aligned}
$$

(iii) With the same argument as above, we can prove $A\left(\mu, \lambda, x_{2}\right)>0$ by dividing $\mu$ into small sections, such as $\mu \in[3.01,3.015), \mu \in[3.0073,3.01)$ and $\mu \in[3.00547,3.0073)$.

As a result, when $3.00547 \leqslant \mu<\lambda \leqslant 1+\sqrt{5}, A\left(\mu, \lambda, x_{2}\right)>0$. Therefore, (2.48) holds for $x=y_{2}$. Let $B(x)=F_{\lambda}\left(F_{\mu}\left(F_{\mu}(x)\right)\right)-x$, then when $x \in\left[\beta_{\mu}, y_{2}\right]$,

$$
\begin{aligned}
B_{x}^{\prime} & =F_{\lambda}^{\prime}\left(F_{\mu}\left(F_{\mu}(x)\right)\right) F_{\mu}^{\prime}\left(F_{\mu}(x)\right) F_{\mu}^{\prime}(x)-1 & & \left(F_{\mu}\left(F_{\mu}(x)\right)<F_{\mu}(x)<x\right) \\
& <F_{\lambda}^{\prime}\left(F_{\mu}\left(F_{\mu}(x)\right)\right)\left(F_{\mu}^{\prime}\left(F_{\mu}\left(F_{\mu}(x)\right)\right)\right)^{2}-1 & & \left(F_{\mu}\left(F_{\mu}(x)\right) \geqslant \beta_{\mu}\right) \\
& <0 . & &
\end{aligned}
$$

So Lemma 2.2.3 holds. 


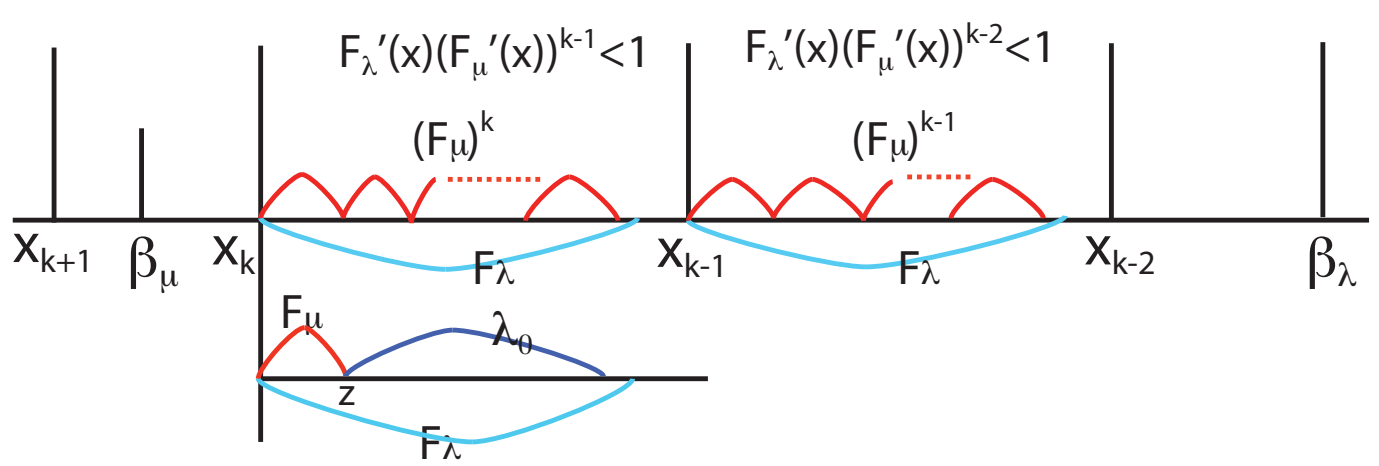

Figure 4.22: $F_{\lambda}\left(x_{k}\right)>y$

Proof of Lemma 2.2.4. We will prove Lemma 2.2.4 in two different cases. When $\frac{\partial F_{\lambda}(x)}{\partial \lambda} \geqslant 0$, we will use the similar method as the case when $1<\mu<\lambda \leqslant 2$. While for the case $\frac{\partial F_{\lambda}(x)}{\partial \lambda}<0$, we will use different method.

Case I. $\frac{\partial F_{\lambda}\left(x_{k}\right)}{\partial \lambda} \geqslant 0$, i.e. $2-3 \lambda x_{k}+3 \lambda x_{k}^{2} \geqslant 0$.

Let $\lambda_{1}<\lambda$ and $\lambda_{2}<\lambda_{1}$ defined by $F_{\lambda_{1}}^{\prime}\left(\beta_{\mu}\right)\left(F_{\mu}^{\prime}\left(\beta_{\mu}\right)\right)^{k-1}=1$ and $F_{\lambda_{2}}^{\prime}\left(\beta_{\mu}\right)\left(F_{\mu}^{\prime}\left(\beta_{\mu}\right)\right)^{k-2}=1$ respectively. We use induction principle. When $\theta \geqslant \lambda_{2}$, for $x \in\left[\beta_{\mu}, y_{k-1}\right]$, we first assume

$$
F_{\theta}(\underbrace{F_{\mu} \cdots F_{\mu}}_{k-1}(x))>x
$$

where $\underbrace{F_{\mu} \cdots F_{\mu}}_{k-1}\left(y_{k-1}\right)=x_{k-1}$.

We now prove that (2.49) holds for $x \in\left[\beta_{\mu}, y_{k}\right]$, i.e.

$$
F_{\lambda}(\underbrace{F_{\mu} \cdots F_{\mu}}_{k}(x))>x
$$

where $\underbrace{F_{\mu} \cdots F_{\mu}}_{k}\left(y_{k}\right)=x_{k}$.

Recall the property of $D(\mu, \lambda, k)$ in the proof of Lemma 2.2.3. Consider $D(\mu, \lambda, k)=1$. We can express $\lambda$ as a function of $\mu$ and then $\lambda^{\prime}(\mu)>0$. When $k=2, \lambda=1+\sqrt{5}$, we have $\mu=3.035$. Note $D(\mu, \lambda, 2)<1$ when $\mu>3.035$, so $D(\mu, \lambda, k)<1$. So we just need to discuss the case when $\mu \in[3.00547,3.035)$.

Let $\lambda_{0} \in\left(\lambda_{2}, \lambda_{1}\right)$. When $\mu$ is in a small interval, it is possible to choose a constant $\lambda_{0}$ (independent of $\mu$ ) such that $F_{\lambda}\left(x_{k}\right)>F_{\lambda_{0}}(z)$ as the figure 4.22 shows, where $F_{\mu}(z)=x_{k}$, so $z=\frac{\mu+\sqrt{\mu^{2}-2 \mu-2 \sqrt{\mu^{2}-4 \mu x_{k}}}}{2 \mu}$. Obviously, $z \in\left(x_{k}, x_{k-1}\right.$ by Lemma 2.46. Note $x_{k}\left(1-x_{k}\right)>$ $z(1-z)=\frac{1}{2 \mu}+\frac{\sqrt{\mu^{2}-4 \mu x_{k}}}{2 \mu^{2}}$. Let $A=F_{\lambda}\left(x_{k}\right)-F_{\lambda_{0}}(z)$, then

$$
\begin{aligned}
A\left(\mu, \lambda, x_{k}\right)= & \lambda^{2} x_{k}\left(1-x_{k}\right)\left(1-\lambda x_{k}+\lambda x_{k}^{2}\right) \\
& -\lambda_{0}^{2}\left(\frac{1}{2 \mu}+\frac{\sqrt{\mu^{2}-4 \mu x_{k}}}{2 \mu^{2}}\right)\left(1-\lambda_{0}\left(\frac{1}{2 \mu}+\frac{\sqrt{\mu^{2}-4 \mu x_{k}}}{2 \mu^{2}}\right)\right) .
\end{aligned}
$$


If we differentiate $A\left(\mu, \lambda, x_{k}\right)$ with respect to $x_{k}$ and $\mu$, we can get

$$
\begin{aligned}
\frac{\partial A\left(\mu, \lambda, x_{k}\right)}{\partial x_{k}}= & \lambda^{2}\left(1-2 x_{k}\right)\left(1-2 \lambda x_{k}+2 \lambda x_{k}^{2}\right) \\
& +\frac{\lambda_{0}^{2}}{\mu \sqrt{\mu^{2}-4 \mu x_{k}}}\left(1-2 \lambda_{0}\left(\frac{1}{2 \mu}+\frac{\sqrt{\mu^{2}-4 \mu x_{k}}}{2 \mu^{2}}\right)\right) \\
\frac{\partial A^{2}\left(\mu, \lambda, x_{k}\right)}{\partial x_{k}^{2}}= & -2 \lambda^{2}\left(6 \lambda x_{k}^{2}-6 \lambda x_{k}+\lambda+1\right)-\frac{2 \lambda_{0}^{2}}{\mu\left(\mu^{2}-4 \mu x_{k}\right)} \frac{\lambda_{0}-\mu}{\sqrt{\mu^{2}-4 \mu x_{k}}} .
\end{aligned}
$$

Note $6 \lambda x^{2}-6 \lambda x+\lambda+1>0$ and $\lambda_{0}>\lambda_{2}>\mu$, so $\frac{\partial A^{2}\left(\mu, \lambda, x_{k}\right)}{\partial x_{k}^{2}}<0$ and $A\left(\mu, \lambda, x_{k}\right)$ reach its minimum value when $x_{k}$ reach its maximum or minimum point. Moreover,

$$
\begin{aligned}
\frac{\partial A\left(\mu, \lambda, x_{k}\right)}{\partial \mu}= & -\lambda_{0}^{2} \frac{-\mu+6 x_{k}-\sqrt{\mu^{2}-4 \mu x_{k}}}{2 \mu^{2} \sqrt{\mu^{2}-4 \mu x_{k}}}\left(1-\lambda_{0}\left(\frac{1}{2 \mu}+\frac{\sqrt{\mu^{2}-4 \mu x_{k}}}{2 \mu^{2}}\right)\right) \\
& +\lambda_{0}^{3}\left(\frac{1}{2 \mu}+\frac{\sqrt{\mu^{2}-4 \mu x_{k}}}{2 \mu^{2}}\right)\left(\frac{-\mu+6 x_{k}-\sqrt{\mu^{2}-4 \mu x_{k}}}{2 \mu^{2} \sqrt{\mu^{2}-4 \mu x_{k}}}\right) \\
= & \lambda_{0}^{2} \frac{-\mu+6 x_{k}-\sqrt{\mu^{2}-4 \mu x_{k}}}{2 \mu^{2} \sqrt{\mu^{2}-4 \mu x_{k}}}\left[2 \lambda_{0}\left(\frac{1}{2 \mu}+\frac{\sqrt{\mu^{2}-4 \mu x_{k}}}{2 \mu^{2}}\right)-1\right] .
\end{aligned}
$$

Considering the following facts,

$$
\begin{aligned}
-\mu+6 x_{k}-\sqrt{\mu^{2}-4 \mu x_{k}} & >-\mu+6 \cdot 0.690988-\sqrt{\mu^{2}-4 \cdot 0.690988 \mu} \\
& >-3.035+4.1459-\sqrt{3.035^{2}-2.76 \cdot 3.035} \\
& =0.1973>0, \\
\lambda_{0}\left(\frac{1}{2 \mu}+\frac{\sqrt{\mu^{2}-4 \mu x_{k}}}{2 \mu^{2}}\right) & >\mu\left(\frac{1}{2 \mu}+\frac{\sqrt{\mu^{2}-4 \mu x_{k}}}{2 \mu^{2}}\right)>\frac{1}{2} .
\end{aligned}
$$

Therefore, $\frac{\partial A\left(\mu, \lambda, x_{k}\right)}{\partial \mu}>0$ and $A\left(\mu, \lambda, x_{k}\right)$ is an increasing function as to $\mu$. So $A\left(\mu, \lambda, x_{k}\right)$ gets the minimum value when $\mu$ is at the lowest value and $x_{k}$ is at endpoints.

Solving the inequality $\frac{\partial F_{\lambda}\left(x_{k}\right)}{\partial \lambda}>0$, we can get $x_{k} \geqslant \frac{1}{2}+\frac{\sqrt{9 \lambda^{2}-24 \lambda}}{6 \lambda}$. Consider $\beta_{\mu} \geqslant$ $\frac{1}{2}+\frac{\sqrt{9 \lambda^{2}-24 \lambda}}{6 \lambda}$, we can get $\mu \geqslant 3.0176$ as $\lambda \leqslant 1+\sqrt{5}$. In other word, if $\mu \geqslant 3.0176$, then $2-3 \lambda x_{k}+3 \lambda x_{k}^{2}>0$. As a result, $F_{\lambda}(x)$ must be an increasing function with respect to $\lambda$ for any $\lambda \in(\mu, 1+\sqrt{5}]$ and $x \in\left(\beta_{\mu}, \beta_{\lambda}\right)$. As a result, when $\mu \geqslant 3.0176$, we can use Case $\mathbf{I}$ to prove. Otherwise, we will use Case I and following Case II to prove.

Case II. $\frac{\partial F_{\lambda}\left(x_{k}\right)}{\partial \lambda}<0$, i.e. $2-3 \lambda x_{k}+3 \lambda x_{k}^{2}<0 . \quad \beta_{\mu} \leqslant x_{k} \leqslant 0.709735$. It follows that $\mu \leqslant 3.0176$. But $\beta_{\mu} \geqslant 0.690988$ when $\mu \geqslant 3.00547$, so $x_{k} \geqslant 0.690988$, and then $\lambda \leqslant 3.1222$. Let $x=F_{\lambda}\left(x_{k}\right)$ and $x_{*}=F_{\mu}(\underbrace{F_{\mu} \cdots F_{\mu}}_{k-1}(x))$, then $x_{k}=\frac{\lambda+\sqrt{\lambda^{2}-2 \lambda-2 \sqrt{\lambda^{2}-4 \lambda x}}}{2 \lambda}$, Since $x-F_{\mu}(x)>F_{\mu}(x)-F_{\mu}\left(F_{\mu}(x)\right)$, we choose $l$ such that $l\left(x-F_{\mu}(x)\right)>x-x_{*}$ and it is easy to see that $l \leqslant k$ as Figure 4.23 shows, then we need to prove the following inequality:

$$
x-l\left(x-F_{\mu}(x)\right)>x_{k}=\frac{\lambda+\sqrt{\lambda^{2}-2 \lambda-2 \sqrt{\lambda^{2}-4 \lambda x}}}{2 \lambda},
$$




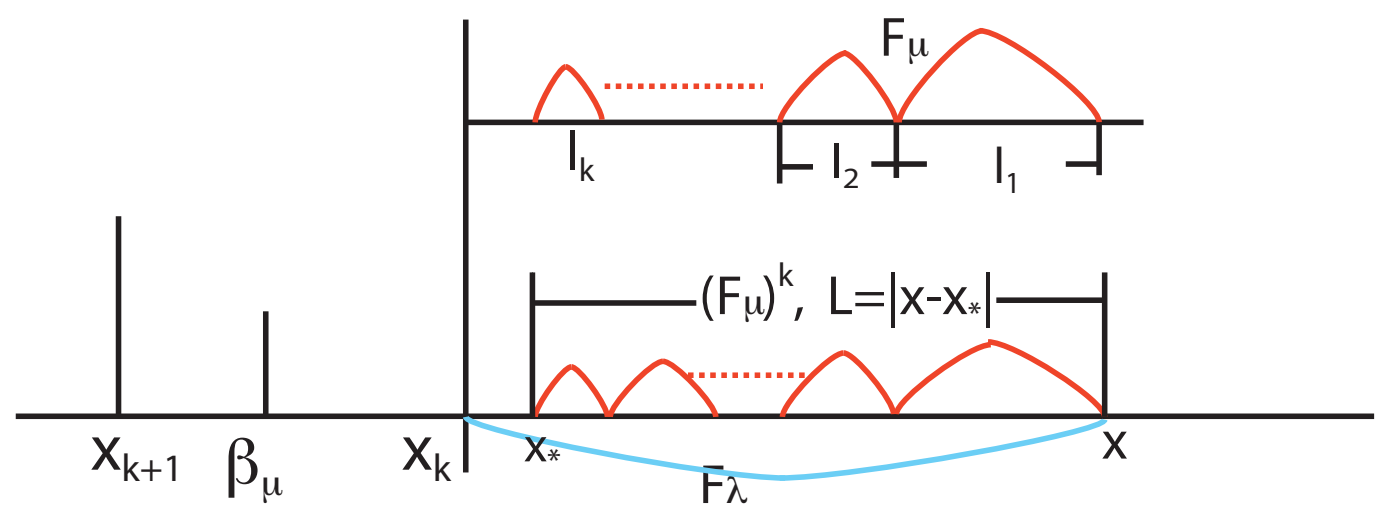

Figure 4.23: $l_{1}=x-F_{\mu}(x)>j l_{2}=j\left(F_{\mu}(x)-F_{\mu}\left(F_{\mu}(x)\right)\right), L=\sum_{i=1}^{k} l_{i}$

Let $M(\mu, \lambda, x, l)=x-l\left(x-F_{\mu}(x)\right)-\frac{\lambda+\sqrt{\lambda^{2}-2 \lambda-2 \sqrt{\lambda^{2}-4 \lambda x}}}{2 \lambda}$. It is obvious that $M(\mu, \lambda, x, l)$ decreases as $l$ increases. Meanwhile, we differentiate $M(\mu, \lambda, x, l)$ with respect to $\mu, \lambda$ and $x$,

$$
\frac{\partial M(\mu, \lambda, x, l)}{\partial \mu}=l x(1-x) \mu\left(2-3 \mu x+3 \mu x^{2}\right) .
$$

Since it is easy to get $2-3 \mu x+3 \mu x^{2}>0$ for $x>0.690988$ and $\mu \leqslant 3.0176$. So $\frac{\partial M(\mu, \lambda, x, l)}{\partial \mu}>0$ and $M(\mu, \lambda, x, l)$ increases as $\mu$ increases. Moreover,

$$
\begin{aligned}
\frac{\partial M(\mu, \lambda, x, l)}{\partial \lambda}= & -\frac{\lambda-6 x+\sqrt{\lambda^{2}-4 \lambda x}}{2 \lambda \sqrt{\lambda^{2}-2 \lambda-2 \sqrt{\lambda^{2}-4 \lambda x} \sqrt{\lambda^{2}-4 \lambda x}}}, \\
\frac{\partial M^{2}(\mu, \lambda, x, l)}{\partial \lambda^{2}}= & \frac{1}{2 \lambda^{2}\left(\lambda^{2}-2 \lambda-2 \sqrt{\lambda^{2}-4 \lambda x}\right)\left(\lambda^{2}-4 \lambda x\right)} \\
& \cdot\left[-\left(2 \lambda x+6 x \sqrt{\lambda^{2}-4 \lambda x}\right) \sqrt{\lambda^{2}-2 \lambda-2 \sqrt{\lambda^{2}-4 \lambda x}}\right. \\
& +\frac{\lambda\left(\lambda-6 x+\sqrt{\lambda^{2}-4 \lambda x}\right)\left[(\lambda-1)\left(\lambda^{2}-4 \lambda x\right)+(\lambda-2 x)\left(\lambda^{2}-2 \lambda-3 \sqrt{\lambda^{2}-4 \lambda x}\right)\right]}{\sqrt{\lambda^{2}-4 \lambda x} \sqrt{\lambda^{2}-2 \lambda-2 \sqrt{\lambda^{2}-4 \lambda x}}} .
\end{aligned}
$$

Let $m_{1}(\lambda, x)=\lambda+\frac{\sqrt{\lambda^{2}-4 \lambda x}}{2}-6 x$, then $m_{1}(\lambda, x) \leqslant m_{1}(1+\sqrt{5}, 0.690988)<0$. So we just need to check the sign of $-2 x\left(\lambda^{2}-2 \lambda-2 \sqrt{\lambda^{2}-4 \lambda x}\right)+0.5\left[(\lambda-1)\left(\lambda^{2}-4 \lambda x\right)+(\lambda-2 x)\left(\lambda^{2}-\right.\right.$ $\left.\left.2 \lambda-3 \sqrt{\lambda^{2}-4 \lambda x}\right)\right]$. Let $m(\lambda, x)=-4 x\left(\lambda^{2}-2 \lambda-2 \sqrt{\lambda^{2}-4 \lambda x}\right)+(\lambda-1)\left(\lambda^{2}-4 \lambda x\right)+(\lambda-$ $2 x)\left(\lambda^{2}-2 \lambda-3 \sqrt{\lambda^{2}-4 \lambda x}\right)$. Then we have the following inequality as $x \geqslant 0.690988$,

$$
\begin{aligned}
m(\lambda, x) & =(\lambda-6 x)\left(\lambda^{2}-2 \lambda-3 \sqrt{\lambda^{2}-4 \lambda x}\right)+\sqrt{\lambda^{2}-4 \lambda x}\left(-4 x+(\lambda-1) \sqrt{\lambda^{2}-4 \lambda x}\right) \\
& <\sqrt{\lambda^{2}-4 \lambda x}\left(-4 x+(\lambda-1) \sqrt{\lambda^{2}-4 \lambda x}\right) \\
& <\sqrt{\lambda^{2}-4 \lambda x}\left(-4 \cdot 0.690988+\sqrt{5} \sqrt{\left.(1+\sqrt{5})^{2}-4(1+\sqrt{5}) 0.690988\right)}\right. \\
& <0 .
\end{aligned}
$$


As a result, $\frac{\partial M^{2}(\mu, \lambda, x, k)}{\partial \lambda^{2}}<0$ and $M(\mu, \lambda, x, k)$ gets the minimum value when $\lambda$ is at the endpoints. Moreover,

$$
\begin{aligned}
\frac{\partial M(\mu, \lambda, x, l)}{\partial x} & =1-l+l \mu^{2}(1-2 x)\left(1-2 \mu x+2 \mu x^{2}\right)-\frac{1}{\sqrt{\lambda^{2}-2 \lambda-2 \sqrt{\lambda^{2}-4 \lambda x}} \sqrt{\lambda^{2}-4 \lambda x}}, \\
\frac{\partial M^{2}(\mu, \lambda, x, l)}{\partial x^{2}} & =-2 l \mu^{2}\left(6 \mu x^{2}-6 \mu x+\mu+1\right)-\frac{2 \lambda\left(\lambda^{2}-2 \lambda-3 \sqrt{\lambda^{2}-4 \lambda x}\right)}{\left(\sqrt{\lambda^{2}-2 \lambda-2 \sqrt{\lambda^{2}-4 \lambda x}}\right)^{3}\left(\sqrt{\lambda^{2}-4 \lambda x}\right)^{3}} .
\end{aligned}
$$

Let $m_{2}(\lambda, x)=\lambda^{2}-2 \lambda-3 \sqrt{\lambda^{2}-4 \lambda x}$, then $m_{2}(\lambda, x)>m_{2}(1+\sqrt{5}, 0.690988)>0$. At the same time, since $6 \mu x^{2}-6 \mu x+\mu+1=6 \mu\left(x-\frac{1}{2}\right)^{2}-\frac{\mu}{2}+1>0$ when $\mu<3.0176$, then we can get $\frac{\partial M^{2}(\mu, \lambda, x, l)}{\partial x^{2}}<0$ and $M(\mu, \lambda, x, l)$ gets the minimum value when $x$ is at the endpoints.

In conclusion, for $\mu \in[3.00547,3.0176], M(\mu, \lambda, x, l)$ increases as $\mu$ increases and $k$ decreases. So $M(\mu, \lambda, x, l)$ reach the lowest point when $x$ and $\lambda$ get the endpoints.

Now we can prove $(2.49)$ for $k=3, \cdots, 21$ respectively. Now we take two examples $k=3$ and $k=6$ to show how to use Case I and Case II. Other computation will be omitted.

When $k=3$. a). When $2-3 \lambda x_{k}+3 \lambda x_{k}^{2} \geqslant 0$, use Case I. Consider $F_{\lambda_{1}}^{\prime}\left(\beta_{\mu}\right) F_{\mu}^{\prime}\left(\beta_{\mu}\right)^{2}=1$ and $F_{\lambda_{2}}^{\prime}\left(\beta_{\mu}\right) F_{\mu}^{\prime}\left(\beta_{\mu}\right)=1$, and

$A\left(\mu, \lambda, x_{3}\right)=\lambda^{2} x_{3}\left(1-x_{3}\right)\left(1-\lambda x_{3}+\lambda x_{3}^{2}\right)-\lambda_{0}^{2}\left(\frac{1}{2 \mu}+\frac{\sqrt{\mu^{2}-4 \mu x_{3}}}{2 \mu^{2}}\right)\left(1-\lambda_{0}\left(\frac{1}{2 \mu}+\frac{\sqrt{\mu^{2}-4 \mu x_{3}}}{2 \mu^{2}}\right)\right)$.

Because $\lambda>\lambda_{1}$, it is easy to see that $A\left(\mu, \lambda, x_{3}\right)>A\left(\mu, \lambda_{1}, x_{3}\right)$. According to the above description, we have the following result.

(i) When $\mu \in[3.0275,3.035]$, obviously we have $x_{3} \in[0.690988,0.72666], \lambda \geqslant \lambda_{1}>$ 3.1838 , and $\lambda_{2}<3.16344$. We choose $\lambda_{0}=3.164>\lambda_{2}$ and then we have

$A\left(\mu, \lambda_{1}, x_{3}\right)>\min \{A(3.0275,3.1838,0.72666), A(3.0275,3.1838,0.690988)\}>0$.

(ii) When $\mu \in[3.023,3.0275]$, then $x_{3} \in[0.690988,0.725], \lambda_{2}<3.12833$ and $\lambda_{1} \geqslant 3.153$.

We choose $\lambda_{0}=3.12833>\lambda_{2}$ and then we have

$$
A\left(\mu, \lambda_{1}, x_{3}\right)>\min \{A(3.023,3.153,0.725), A(3.023,3.153,0.690988)\}>0 .
$$

(iii) When $\mu \in[3.0195,3.023]$, then $x_{3} \in[0.690988,0.723961], \lambda_{2}<3.10742$ and $\lambda_{1} \geqslant$ 3.12951. We choose $\lambda_{0}=3.10742>\lambda_{2}$ and then we have

$A\left(\mu, \lambda_{1}, x_{3}\right)>\min \{A(3.0195,3.12951,0.723961), A(3.0195,3.12951,0.690988)\}>0$.

(iv) When $\mu \in[3.0171,3.0195]$, then $x_{3} \in[0.690988,0.723151], \lambda_{2}<3.09121$ and $\lambda_{1} \geqslant$ 3.11347. We choose $\lambda_{0}=3.09121>\lambda_{2}$ and then we have

$A\left(\mu, \lambda_{1}, x_{3}\right)>\min \{A(3.0171,3.11347,0.723151), A(3.0171,3.11347,0.690988)\}>0$. 
(v) When $\mu \in[3.013,3.0171]$, then $x_{3} \in[0.690988,0.72259], \lambda_{2}<3.08011$ and $\lambda_{1} \geqslant$ 3.086. We choose $\lambda_{0}=3.08011>\lambda_{2}$ and then we have

$$
\begin{gathered}
A\left(\mu, \lambda, x_{3}\right)>A(3.013,3.106,0.72259)>0, \quad \lambda \in[3.106,1+\sqrt{5}], x_{3} \leqslant 0.72259, \\
A\left(\mu, \lambda, x_{3}\right)>A(3.013,3.086,0.71003)>0, \quad \lambda \in[3.086,3.106], x_{3} \leqslant 0.70813 . \\
A\left(\mu, \lambda, x_{3}\right)>\quad \min \{A(3.013,3.106,0.72259), A(3.013,3.086,0.71003), \\
A(3.013,3.086,0.690988)\}>0 .
\end{gathered}
$$

(vi) When $\mu \in[3.01,3.013]$, then $x_{3} \in[0.690988,0.7222], \lambda_{2}<3.0612$ and $\lambda_{1} \geqslant 3.066$. We choose $\lambda_{0}=3.0612>\lambda_{2}$ and then we have

$$
\begin{gathered}
A\left(\mu, \lambda, x_{3}\right)>A(3.01,3.096,0.7222)>0, \quad \lambda \in[3.096,1+\sqrt{5}], x_{3} \leqslant 0.72259, \\
A\left(\mu, \lambda, x_{3}\right)>A(3.01,3.066,0.70536)>0, \quad \lambda \in[3.066,3.096], x_{3} \leqslant 0.70536 . \\
A\left(\mu, \lambda, x_{3}\right)>\quad \min \{A(3.01,3.096,0.7222), A(3.01,3.066,0.70536), \\
A(3.01,3.066,0.690988)\}>0 .
\end{gathered}
$$

(vii) When $\mu \in[3.0075,3.01]$, then $x_{3} \in[0.690988,0.7209], \lambda_{2}<3.0473$ and $\lambda_{1} \geqslant 3.05$. We choose $\lambda_{0}=3.0473>\lambda_{2}$ and then we have

$$
\begin{aligned}
A\left(\mu, \lambda, x_{3}\right)>A(3.0075,3.12,0.7209)>0, & \lambda \in[3.12,1+\sqrt{5}], x_{3} \leqslant 0.7209, \\
A\left(\mu, \lambda, x_{3}\right)>A(3.0075,3.06,0.7079)>0, & \lambda \in[3.06,3.12), x_{3} \leqslant 0.7079, \\
A\left(\mu, \lambda, x_{3}\right)>A(3.0075,3.05,0.6982)>0, & \lambda \in[3.05,3.06), x_{3} \leqslant 0.6982 . \\
A\left(\mu, \lambda, x_{3}\right)> & \min \{A(3.0075,3.12,0.7209), A(3.0075,3.06,0.7079), \\
& A(3.0075,3.05,0.6982), A(3.0075,3.05,0.690988)>0\} .
\end{aligned}
$$

(viii) When $\mu \in[3.006,3.0075]$, then $x_{3} \in[0.690988,0.720271], \lambda_{2}<3.03561$ and $\lambda_{1} \geqslant$ 3.04. We choose $\lambda_{0}=3.03561>\lambda_{2}$ and then we have

$$
\begin{aligned}
& A\left(\mu, \lambda, x_{3}\right)>A(3.006,3.065,0.720271)>0, \quad \lambda \in[3.065,1+\sqrt{5}], x_{3} \leqslant 0.720271, \\
& A\left(\mu, \lambda, x_{3}\right)>A(3.006,3.04,0.69811)>0, \quad \lambda \in[3.05,3.065), x_{3} \leqslant 0.69811, \\
& A\left(\mu, \lambda, x_{3}\right)>\min \{A(3.006,3.065,0.720271), A(3.006,3.04,0.69811), \\
& A(3.006,3.04,0.690988)\}>0 .
\end{aligned}
$$

(ix) When $\mu \in[3.00547,3.006]$, then $x_{3} \in[0.690988,0.7199], \lambda_{2}<3.0286$ and $\lambda_{1} \geqslant$ 3.03661. We choose $\lambda_{0}=3.0286>\lambda_{2}$ and then we have

$$
\begin{aligned}
& A\left(\mu, \lambda, x_{3}\right)>A(3.00547,3.09,0.7199)>0, \quad \lambda \in[3.09,1+\sqrt{5}], x_{3} \leqslant 0.7199, \\
& A\left(\mu, \lambda, x_{3}\right)>A(3.00547,3.03661,0.702)>0, \quad \lambda \in[3.03661,3.09), x_{3} \leqslant 0.7079, \\
& A\left(\mu, \lambda, x_{3}\right)>\min \{A(3.00547,3.09,0.7199), A(3.00547,3.03661,0.702), \\
& A(3.00547,3.03661,0.690988)\}>0 .
\end{aligned}
$$


b). When $2-3 \lambda x_{k}+3 \lambda x_{k}^{2}<0$, use Case II. $x_{3} \leqslant 0.709755$. We can also get $\lambda \leqslant$ 3.144778 for $\mu \in[3.00547,3.0176]$ and $x \leqslant 0.7176$. Recalling the property of $M(\mu, \lambda, x, l)$, we can get

$$
\begin{aligned}
M(\mu, \lambda, x, l)> & \min \{M(3.00547,3.144778,0.690988,3), M(3.00547,3.1222,0.690988,3), \\
& M(3.00547,3.1222,0.7176,3), M(3.00547,3.144778,0.7176,3)\}>0 .
\end{aligned}
$$

When $k=6$.

1. Assume $\frac{\partial F_{\lambda}\left(x_{k}\right)}{\partial \lambda}>0$. Define $\lambda_{1}$ and $\lambda_{2}$ by $F_{\lambda_{1}}^{\prime}\left(\beta_{\mu}\right) F_{\mu}^{\prime}\left(\beta_{\mu}\right)^{5}=1$ and $F_{\lambda_{2}}^{\prime}\left(\beta_{\mu}\right) F_{\mu}^{\prime}\left(\beta_{\mu}\right)^{4}=$ 1 , and

$$
\begin{aligned}
A\left(\mu, \lambda, x_{6}\right)= & \lambda^{2} x_{6}\left(1-x_{6}\right)\left(1-\lambda x_{6}+\lambda x_{6}^{2}\right) \\
& -\lambda_{0}^{2}\left(\frac{1}{2 \mu}+\frac{\sqrt{\mu^{2}-4 \mu x_{6}}}{2 \mu^{2}}\right)\left(1-\lambda_{0}\left(\frac{1}{2 \mu}+\frac{\sqrt{\mu^{2}-4 \mu x_{6}}}{2 \mu^{2}}\right)\right) .
\end{aligned}
$$

Recall the property of $D(\mu, \lambda, k)$, solving $F_{\lambda_{1}}^{\prime}\left(\beta_{\mu}\right) F_{\mu}^{\prime}\left(\beta_{\mu}\right)^{5}<1$ and $F_{\lambda_{1}}^{\prime}\left(\beta_{\mu}\right) F_{\mu}^{\prime}\left(\beta_{\mu}\right)^{6}<$ 1 to get $\mu>3.0182$ and $\mu>3.0156$. Recall that $A(\mu, \lambda, k)$ must be increasing in $\lambda$ for $\lambda \in(\mu, 1+\sqrt{5}]$ when $\mu>3.0176$. So when $\mu \in[3.0176,3.0182]$, or when $\mu<3.0176$ but $2-3 \lambda x_{k}+3 \lambda x_{k}^{2}>0$, then with the same argument as above, we can also prove $A\left(\mu, \lambda, x_{k}\right)>0$ and get (4.47).

2. When $\frac{\partial F_{\lambda}\left(x_{k}\right)}{\partial \lambda}<0$. While for $\mu \in[3.00547,3.0176)$, We can calculate the range of $x_{k}$ and $\lambda$ as follows. When $2-3 \lambda x_{k}+3 \lambda x_{k}^{2}<0, \beta_{\mu}<\beta(3.0176)=0.709755$, so $x_{k}<0.709755$. So $x_{k} \in[0.690988,0.709755]$ and $k \geqslant 6$. While for $x_{k}>0.690988$, we have $2-3 \lambda x_{k}+3 \lambda x_{k}^{2}>0$ when $\lambda \leqslant \frac{2}{3\left(x_{k}-x_{k}^{2}\right)}<3.1222$. So $\lambda \in[3.1222,1+\sqrt{5}]$. Since it is easy to get

$$
M(3.00547,1+\sqrt{5}, 0.690988,6)>0, \quad M(3.00547,3.1222,0.690988,6)>0,
$$

so we just need to check the sign of $M(\mu, \lambda, x, l)$ when $x$ gets the maximum point. First note $x=F_{\lambda}\left(x_{k}\right)<0.71916$ for $x_{k} \leqslant 0.709755$.

Let $N(\mu, x, j)=\frac{\mu+\sqrt{\mu^{2}-2 \mu-2 \sqrt{\mu^{2}-4 \mu x}}}{2 \mu}-x-j\left(x-F_{\mu}(x)\right)$. As Figure 4.23 shows, $l_{i}>l_{i+1},(i=1, \cdots, k-1)$ is obvious and then $j>1$. Then

$$
\begin{gathered}
\frac{\partial N(\mu, x, j)}{\partial \mu}=\frac{\mu-6 x+\sqrt{\mu^{2}-4 \mu x}}{2 \mu \sqrt{\mu^{2}-2 \mu-2 \sqrt{\mu^{2}-4 \mu x}} \sqrt{\mu^{2}-4 \mu x}}+j \mu x(1-x)\left(2-3 \mu x+3 \mu x^{2}\right), \\
\frac{\partial N^{2}(\mu, x, j)}{\partial \mu \partial x}=-\frac{\left(3+\frac{\mu}{\sqrt{\mu^{2}-4 \mu x}}\right)\left(\mu^{2}-2 \mu-2 \sqrt{\mu^{2}-4 \mu x}\right)(\mu-4 x)}{\left(\mu^{2}-2 \mu-2 \sqrt{\mu^{2}-4 \mu x}\right)^{\frac{3}{2}}\left(\mu^{2}-4 \mu x\right)^{\frac{3}{2}}} \\
-\frac{\left(\mu-6 x+\sqrt{\mu^{2}-4 \mu x}\right)\left(-\mu^{2}+2 \mu+3 \sqrt{\mu^{2}-4 \mu x}\right)}{\left(\mu^{2}-2 \mu-2 \sqrt{\mu^{2}-4 \mu x}\right)^{\frac{3}{2}}\left(\mu^{2}-4 \mu x\right)^{\frac{3}{2}}} \\
+2 j x(1-x)\left(1-3 \mu x+3 \mu x^{2}\right)<0 .
\end{gathered}
$$


Recall that $-\mu+6 x_{k}-\sqrt{\mu^{2}-4 \mu x_{k}}>0$ and $-\mu+6 x-\sqrt{\mu^{2}-4 \mu x}$ increases as $x$ increases, so $\mu-6 x+\sqrt{\mu^{2}-4 \mu x}<0$. We also have $\mu^{2}-2 \mu-3 \sqrt{\mu^{2}-4 \mu x}>0$, $3 \mu x^{2}-3 \mu x+1<0$ for $x<0.71916$. Therefore, $\frac{\partial N^{2}(\mu, x, j)}{\partial \mu \partial x}<0$ and then $\frac{\partial N(\mu, x, j)}{\partial \mu}$ decreases in $x$. So for $x>\beta_{\mu}$, it is easy to get

$$
\frac{\partial N(\mu, x, j)}{\partial \mu}<\frac{\partial N\left(\mu, \beta_{\mu}, j\right)}{\partial \mu}=0 .
$$

Consequently, we have $\frac{\partial N(\mu, x, j)}{\partial \mu}<0$ and $N(\mu, x, j)$ decreases in $\mu$.

When $k=6$, we will demonstrate in the following that it is sufficient to take $j=1$ in the case $(a)$ and $(b)$. But in other cases, we take different valve of $j$.

(a) $\mu \in[3.013,3.0176]$, then $\lambda>3.166$, and

$$
\begin{aligned}
M(\mu, \lambda, x, l)> & \min \{M(3.013,1+\sqrt{5}, 0.71916,6), \\
& M(3.00547,3.166,0.71916,6)\}>0 .
\end{aligned}
$$

(b) $\mu \in[3.011,3.013)$, then $x_{k} \in[0.700938,0.708483]$ and $\lambda>\frac{2}{3\left(x_{k}-x_{k}^{2}\right)}>3.18$, so $x \in(0.706,0.71731)$ and then we have

$$
\begin{aligned}
M(\mu, \lambda, x, l)> & \min \{M(3.011,1+\sqrt{5}, 0.71731,6), \\
& M(3.011,3.18,0.71731,6)\}>0 .
\end{aligned}
$$

(c) $\mu \in[3.009,3.011)$ and then $x_{k} \in[0.69944,0.70771] . \lambda>\frac{2}{3\left(x_{k}-x_{k}^{2}\right)}>3.172$. So $x \in[0.703823,0.716188]$. We can get $N(\mu, \lambda, x, 1.05)>0$ and then $l=\frac{1-j^{-k}}{1-j^{-1}}=$ 5.329. We can get

$$
\begin{aligned}
M(\mu, \lambda, x, l)> & \min \{M(3.009,1+\sqrt{5}, 0.716188,5.329), \\
& M(3.009,3.172,0.716188,5.329)\}>0 .
\end{aligned}
$$

(d) $\mu \in[3.007,3.009)$ and then $x_{k} \in[0.694127,0.706915] . \lambda>\frac{2}{3\left(x_{k}-x_{k}^{2}\right)}>3.14$. So $x \in[0.69578,0.71505]$.

For $\lambda \in[3.18,1+\sqrt{5}], x_{k} \in[0.70224,0.706915]$ and $x \in[0.70802,0.71505] \mathrm{We}$ can get $N(\mu, x, 1.05)>0$ and then $l=\frac{1-j^{-k}}{1-j^{-1}}=5.329$ and then

$$
\begin{aligned}
M(\mu, \lambda, x, l)> & \min \{M(3.007,1+\sqrt{5}, 0.71505,5.329), \\
& M(3.007,3.18,0.71505,5.329)\}>0 .
\end{aligned}
$$

For $\lambda \in[3.14,3.18), x_{k} \in[0.694127,0.70313]$ and $x \in[0.695776,0.7097]$ We can get $N(\mu, \lambda, x, 1)>0$ and then $l=6$ and then

$$
\begin{aligned}
M(\mu, \lambda, x, l)> & \min \{M(3.007,1+3.14,0.7097,5.329), \\
& M(3.007,3.18,0.7097,5.329)\}>0 .
\end{aligned}
$$


(e) $\mu \in[3.00547,3.007), x_{k} \in[0.690988,0.70611] . \lambda>\frac{2}{3\left(x_{k}-x_{k}^{2}\right)}>3.1222$.

For $\lambda \in[3.175,1+\sqrt{5}), x_{k} \in[0.70154,0.70611]$ and $x \in[0.706976,0.713903]$ We can get $N(\mu, x, 1.075)>0$ and then $l=5.0459$. Consequently, we have

$$
\begin{aligned}
M(\mu, \lambda, x, l)> & \min \{M(3.00547,1+\sqrt{5}, 0.713903,5.046), \\
& M(3.00547,3.18,0.713903,5.046)\}>0 .
\end{aligned}
$$

For $\lambda \in[3.1222,3.175), x_{k} \in[0.690988,0.7018612]$ and $x \in[0.6931,0.707964]$ We can get $N(\mu, x, 1)>0$ and then $l=6$. Consequently, we have

$$
\begin{aligned}
M(\mu, \lambda, x, l)> & \min \{M(3.00547,3.1222,0.707964,6), \\
& M(3.00547,3.175,0.707964,6)\}>0 .
\end{aligned}
$$

Consequently, when $k=6,(2.49)$ holds.

In summary, for $k \in[3,21]$, when $2-3 \lambda x_{k}+3 \lambda x_{k}^{2} \geqslant 0$, we prove $A\left(\mu, \lambda, x_{k}\right)>0$ and then (4.47) holds for $x=y_{k}$. With the same argument as before, (2.49) holds. While when $2-3 \lambda x_{k}+3 \lambda x_{k}^{2}<0$, we prove $M(\mu, \lambda, x, l)>0$. As a result, cen22 also holds. In a word, for $3.00547 \leqslant \mu<\lambda \leqslant 1+\sqrt{5}$, Lemma 2.2 .4 holds. 


\section{Bibliography}

[1] K. T. Alligood, T. D. Sauer and J. A. Yorke, Chaos: an introduction to dynamical systems, SpringerVerlag, 2000.

[2] H. Amann, Ordinary differential equations, An Introduction to Nonlinear Analysis, Walter de Gruyter, Berlin, New York, 1990.

[3] J. Andres, Randomization of Sharkovskii-type theorems, Proceedings of the American Mathematical Society, Volume 136, Number 4, April 2008, Pages 1385-1395.

[4] L. Arnold, Random Dynamical Systems, Springer-verlag, Berlin, Heidelberg, 1998.

[5] V. I. Arnold (Ed.) Dynamical Systems, Springer-verlag, Berlin, Heidelberg, 1994.

[6] D. K. Arrowsmith and C. M. Place, An introduction to dynamical systems, Cambridge University Press, 1990.

[7] K. B. Athreya and J. Dai, Random logistic maps, Journal of Theoretical Probability, Vol.13, No.2 (2000), 595-608.

[8] J. Bank, V. Dragan and A. Jones, Chaos a mathematical introduction, Cambridge University Press (2003).

[9] I. Bendixson, Sur les curbes denies par des equation differentielles, Acta Math. 24 (1901), 1-88.

[10] R. N. Bhattacharya and M. Majumdar, On a theorem of Dubins and Freedman, Journal of Theoretical Probability, Vol.12, No.4, 1999.

[11] R. Bhattacharya and M.Majumdar, Random dynamical systems: a review, Economic Theory (2004).

[12] R. N. Bhattacharya and B. V. Rao, Random iterations of two quadratic maps, In: Cambanies, S.Ghosh, J.K.Karandikar, R.L.,Sen,p.k. Stochastic Processes: A Festschrift in Honour of Gopinath Kallianpur, pp.13-21, Berlin Heidelberg New York:Springer 1993.

[13] T. Caraballo, P. E. Kloeden and J. Real, Discretization of asymptotically stable stationary solutions of delay. J.Dynam. Differential Equations, Vol.18, No.4 (2006), 863-880.

[14] T. Caraballo, P. E. Kloeden and B. Schmalfuss, Exponentially stable stationary solutions for stochastic evolution equations and their perturbation. Appl. Math. Optim., Vol.50 (2004), 183207.

[15] S. N. Chow and J. K. Hale, Methods of bifurcation Theory, Springer-verlag, New York, 1982.

[16] P. Collet and J. P. Eckman, Iterated maps on the interval as dynamical systems, Birkhauser, Bostoon (1980).

[17] J. Duan, K. Lu and B. Schmalfuss, Invariant manifolds for stochastic partial differential equations. Ann.Probab.,Vol31(2003), 2109-2135. 
[18] J. Duan, K. Lu and B. Schmalfuss, Smooth stable and unstable manifolds for stochastic evolutionary equations. J.Dynam.Differential Equations, Vol.16 (2004),949-972.

[19] L. E. Dubins and D. A. Freedman, Invariant probabilities for certain Markov processes, Ann.Math.Statist, Vol(37) (1966), 837-847.

[20] W. E, K. Khanim, A. Mazel and Ya.Sanai, Invariant measures for Burgers equation with stochastic forcing. Ann. of Math., Vol.151(2000), 877-960.

[21] C. R. Feng, H. Z. Zhao and B. Zhou, Pathwise random periodic solutions of stochastic differential equations, Submitted, Preprint, 2010.

[22] R. C. Hilborn, Chaos and Nonlinear Dynamics, pp.195-201, Oxford University Press, 2 edition, 2000 .

[23] Y. Kifer, Random perturbations of dynamical systems. Progress in Probability and Statistics, 16.Birkhauser, Boston, 1988.

[24] H. Kunita, Stochastic flows and stochastic differential equations. Cambridge University Press (1990).

[25] T. Y. Li and J. A. Yorke, Period three implies chaos, American Mathematical Monthly, Vol82 (1975) 985-992.

[26] W. Li and K. Lu, Sternberg theorems for random dynamical systems. Communications on Pure and Applied Mathamatics, Vol.LVIII (2005),941-988.

[27] A.Lienard, Etude des oscillations entretenues, Rev,Gen. d'Elect.23 (1928), 901-902.

[28] P. D. Liu and M. Qian, Smooth ergodic theory of random dynamical systems. Springer, Berlin, 1995.

[29] Y. Liu and H. Z. Zhao, Representation of pathwise stationary solutions of stochastic Burgers equations, Stochastic and Dynamics, Vol9, No.4 (2009), 613-634.

[30] E. N. Lorenz, Deterministic nonperiodic flow, Journal of The Atmosphere Sciences, Vol.20 (1963), 130-141.

[31] S. E. A. Mohammed, T. Zhang and H. Z. Zhao, The stable manifold theorem for semi-linear stochastic evolution equations and stochastic partial differential equations. Mem. Amer. Math. Soc., Vol.196 (2008), No. 917, 1-105.

[32] E. Mosekilde, Topics in nonlinear dynamics, World Scientific Publishing(1998).

[33] G. Ochs and V. I. Oseledets, Topological fixed point theorems do not hold for random dynamical systems, Journal of Dynamics and Differential Equations, Vol.11 (1999).

[34] H. Poincare, Memoire sur les courbs definier par une equation differentiate, J.Math. Pures Appl.(3)7(1881) 375-422, J.Math. Pures Appl.(3) 8 (1882) 251-296, J.Math. Pures Appl.(4) 1 (1885) 167-244, J.Math. Pures Appl.2 (1886) 151-217.

[35] B. Van der Pol, On relaxation-oscillations, Phil. Mar. 2 (Nov.1926), 978-992.

[36] Ya. Sinai, Two results concerning asympotic behaviour of solutions of Burgers equation with force. J.Statist.Phys., Vol.64 (1991), 1-12.

[37] Ya. Sinai, Burgers system driven by a periodic stochastic flows. In: Itố $s$ stochastic calculus and probability theory, Springer, Tokyo (1996), 347-353.

[38] J. C. Sprott, Chaos and time-series analysis, Oxford University Press(2003).

[39] S. H. Strogatz, Nonlinear dynamics and chaos, Perseus Puliblishing(2000). 
[40] S. M. Ulam and J. von Neumann, Random ergodic theorems. Bull. Amer. Math. Soc.51(1945), 660.

[41] S. Wiggins, Global bifurcation and chaos, Springer-Verlag, Newyork, 1988.

[42] Q. Zhang and H. Z. Zhao, Stationary solutions os SPDEs and infinite horizon BDSDEs. Journal of Functional Analysis, Vol252 (2007), 171-219.

[43] Q. Zhang and H. Z. Zhao, Stationary solutions os SPDEs and infinite horizon BDSDEs with NonLipschitz coefficients. Journal of Differential Equations, Vol248 (2010) 953-991.

[44] H. Z. Zhao and Z. H. Zheng, Random periodic solutions of random dynamical systems, Journal of Differential Equations, vol. 246 (2009), 2020-2038. 